\title{
EARLY HISTORY OF HEAVY ISOTOPE RESEARCH AT BERKELEY
}

AUGUST, 1940 to APRIL, 1942

Glenn T. Seaborg

June 1976

For Referencis?

Not to be taken from this rosm 
EARLY HISTORY OF HEAVY

ISOTOPE RESEARCH AT BERKELEY

August 1940 to April 1942

by Glenn T. Seaborg

Prepared for the U.S. Energy Research and Development Administration under Contract W-7405-ENG-48 
INTRODUCTION

I have had the idea for some time that it would be interesting and worthwhile to put together an account of the early work on heavy isotopes at Berkeley. Of a special interest is the discovery of plutonium (atomic number 94) and the isotope $\mathrm{U}^{233}$, and the demonstration of their fission with slow neutrons. This work served as a prelude to the subsequent Plutonium Project (Metallurgical Project) centered at the University of Chicago, in connection with which I have also had the idea of putting together a history of the work of my chemistry group.

I have decided that it would be an interesting challenge to write this account on a day-to-day basis in a style that would be consistent with the entries having been written at the end of each day. The aim would be to make this history as accurate as possible by going back to the original records and using them with meticulous care.

A logical starting point is the day when we made our first neutron bombardment of uranium to produce a sample of 2.3 -day $93^{239}$ in order to begin to study its chemical properties by the tracer technique-namely, August 30, 1940. A logical termination date would be April 17, 1942the day I departed Berkeley to take up work at the University of Chicago Metallurgical Laboratory.

Fortunately, a large quantity of records is available. By far the most important of these is the laboratory notebooks of the participants in this program, but in addition I have found in my files all my original correspondence, copies of the early reports submitted to the U.S. Uranium Committee and the Plutonium Project, reports submitted to me by the participants, numerous folders full of sheets of additional laboratory data not entered in notebooks, graduate student $\mathrm{Ph} . \mathrm{D}$. theses and related publications, records of purchase orders, inventories of equipment, and so forth.

In searching for and inquiring among my co-workers about the whereabouts of our various laboratory notebooks I have found that many of them are "missing." Fortunately, however, essentially all of those concerned with the early work on element 94 (that is, Arthur C. Wahl's notebooks and a notebook kept by Joseph w. Kennedy, Emilio Segrè and me) and on $\mathrm{U}^{233}$ (that is, John W. Gofman's and Raymond W. Stoughton's notebooks) are available and have been placed at my disposal by these colleagues. I have the feeling that many of the "missing" notebooks are still in existence somewhere and look forward with keen anticipation to finding them someday in order to check how well I have divined their contents through the use of reports, letters, cross references in the available notebooks, and so forth.

This notebook matter deserves a few words of comment. I apparently devised a notebook numbering system for the participants in our group that enables me to make some guesses about the numbers of the missing notebooks. Notebook numbers 1 to 9 were apparently reserved for Kennedy and me, although we never got beyond notebook number 1 . Notebook numbers 
10 to 19 were reserved for Wahl; covering the time span of interest, we have his notebook numbers 10 to 16 inclusive. Friedlander's notebooks are all missing but I deduce that notebook numbers 20 to 29 were reserved for him; I can check this because there is a cross reference in one of Stoughton's notebooks to Friedlander's notebook number 23 . Notebook numbers 30 to 39 were reserved for Gofman and we have available his notebook numbers 32 to 35 , inclusive, covering this period, and extensive reproductions of pages from his notebook numbers 30 and 31 which were available in the Patent Office of the U.S. Atomic Energy Commission. Proceeding chronologically with respect to the date of their start to work with our group, I deduced that Spofford G. English had notebook numbers in the 40s, which was confirmed when I found, during my research on Metallurgical Laboratory papers, a reference to English notebook number 42. I would then deduce that Frederick J. Leitz had notebook numbers in the $50 \mathrm{~s}$, John $w$. Hamaker in the $60 \mathrm{~s}$, and Robert B. Duffield in the 70s. Then we have Stoughton's notebooks with numbers 80 to 82 , inclusive, covering this period. I would deduce that Morris Perlman's missing notebooks have the number range in the $90 \mathrm{~s}$. In my review of Metallurgical Laboratory documents, I find a reference to Isadore Perlman's California notebook number 121, indicating that his sequence of notebooks started with number 120 . I do not know whose notebooks started with number 100 and with number 110 , but presumably Beppino J. Fontana would be one of these. I did have the advantage of using the excellent notebook of Rene J. Prestwood (bearing the simple number 1) which was very helpful. I have the impression, on the basis of reference to Metallurgical Laboratory documents, that many of the notebooks disappeared into the labyrinth of Plutonium Project patent files; hopefully, they may be recoverable at some future date after further more thorough searches.

As I have indicated, a great effort has been made to have accurate entries, both with respect to their content and the date on which the work was done. In a few instances where I used secondary sources, such as reports, the information is accurate but I have had to make an estimate as to the precise dates on which the work was done. This corresponds to only a few percent of the entries and the dates should usually be accurate to within a few days. In very rare instances I have relied on my memory and those of my colleagues, but I have only done this when at least two of us have the same recollection and I have limited such entries to rather notable events that might reasonably have lived in our memories.

Working my way through the notebooks has been an interesting and even stimulating experience. I have even found it exciting on the many occasions when, through a kind of detective work, it was possible to cross check information from several sources and find consistent results.

I hope that I have succeeded in recapturing some of the drama of these early experiments and that my account will have some historical value.

I want to express my appreciation to Peggy Yamada for her capable help in preparing this manuscript and guiding it through the publication procedure. 
This afternoon we irradiated with neutrons our first sample of uranium to produce the recently discovered isotope $93^{239}$ in order to commence a research program to study by the tracer technique the chemical properties of element 93. These chemical investigations are being carried out at my suggestion by one of my graduate students, Arthur $C$. Wahl, who is starting his second year of graduate work under my direction. I believe that this research and its possible expansion into the search for the next transuranium element, element 94, might provide a very suitable subject for Wahl's Ph.D. thesis. Joseph W. Kennedy, who like me is an instructor in the Department of Chemistry here in. Berkeley at the University of California, is collaborating with us on this research. Our work is being carried on in laboratories on the third floor of Gilman Hall, one of the chemistry buildings on the Berkeley campus. The chemical experiments are being done in room 307 where Wahl and my other second year graduate student, Gerhart Friedlander, are working. Our counting equipment is in room 303, which also serves as an office for Kennedy and me.

The discovery of the first transuranium element with the atomic number 93 in the form of the beta-particle emitting isotope $93^{239}(2.3$ days half-life), produced by the bombardment of uranium with neutrons, was announced by Edwin M. McMillan and Philip H. Abelson in a letter to the editor of Physical Review recently. (The June 15, 1940 issue, Vol. 57, p. 1185). They made the interesting observation that this new element has little if any resemblance to its homolog rhenium, but has properties similar to those of uranium, suggesting that there may be a second "rare earth" group of similar elements starting with uranium. They showed that element 93 in a reducing solution (presence of $\mathrm{SO}_{2}$ ) coprecipitates with cerium fluoride, while in an oxidizing solution (presence of bromate ion) it is soluble in the presence of cerium fluoride. These properties provide in principle a method for separating element 93 from other transmutation products through the use of oxidation-reduction cycles in which rare earth fluorides alternately are precipitated without and with the coprecipitation of element 93 radioactivity. This procedure can be termed the rare earth fluoride "oxidation-reduction cycle" for the isolation of element 93.

Our first bombardment, this afternoon, involved the use of 5.5 grams of uranyl nitrate hexahydrate $\left(\mathrm{UO}_{2}\left(\mathrm{NO}_{3}\right)_{2} \cdot 6 \mathrm{H}_{2} \mathrm{O}\right.$, UNH) placed directly behind a beryllium target bombarded with $16 \mathrm{Mev}$ deuterons in the 60-inch cyclotron. This neutron-bombarded uranyl nitrate hexahydrate sample was dissolved in water and the oxidation-reduction cycle was performed by wahl in order to isolate the $93^{239}$ from uranium and fission product radioactivity in order to characterize its radiation and follow its decay. The $93^{239}$ fraction will be followed for decay to see if it displays the. known half-life of $93^{239}, 2.3$ days, and absorption measurements of the radiations in aluminum will be made.

For the measurement of the radiations from our radioactive samples, we have on hand Geiger-Mueller counters and Lauritsen quartz-fiber electroscopes. Our Geiger-Mueller counters are of the conventional cylindrical type with a wire along the axis and are given number designations 
with the letter $z$; e.g., Geiger-Mueller counter no. z-1, z-2, etc., as newly built counters come into operation. (Our first counter, Z-1, was built by Kennedy in the spring of 1938 and he has subsequently built 12 counters with the most recent designated as no. Z-13.) After amplification, the output from the Geiger-Mueller counter is recorded by a scale of eight circuit with a cenco counter. The cylindrical outside container of the air-filled Lauritsen quartz-fiber electroscope is made of aluminum with a window of thickness about $3 \mathrm{mg} / \mathrm{cm}^{2}$ at the bottom for the entrance of the radiations from the sample; we add a sample holder arrangement consisting of a number of slots at increasing distances from the window to allow the interposition of radiation absorbers when the sample is placed in a lower slot. The rate of discharge is measured in terms of the rate of movement of the quartz fiber so the intensity of radioactive samples is recorded in terms of "divisions per second." we number our electroscopes simply as no. 1 , no. 2, etc., as newly acquired instruments are put into operation. We have four electroscopes in operation now.

In order to have a practicable system of distinguishing between this and the subsequent bombardments and the various chemical fractions separated in each bombardment, we intend to use the following nomenclature. Because our primary product of interest is element 93, our designation in each case will start with 93 followed in turn by a number designating the bombardment and a second number designating the chemical fraction within that bombardment. Thus, this first bombardment is designated 93-1 and the chemical fractions that were separated are designated 93-1-1, 93-1-2, and so forth. The purified element 93 fraction upon which the decay and radiation measurements were made was designated 93-1-3.

Monday, September 23, 1940

John W. Gofman and Spofford G. English, two graduate students who are starting at Berkeley this fall, have elected to carry out their graduate work under my direction. English has decided that he will not be very active in research during the first semester, but Gofman has elected to begin his research program immediately. Gofman will work in room 307. (In addition to supervision of graduate student research, my teaching assignment consists of teaching two freshman chemistry laboratory sections.)

Gofman has accepted my suggestion that for his thesis problem he undertake the search for the missing $4 \mathrm{n}+1$ heavy nuclei and the measurement of their radioactive properties. He will start with an investigation of $\mathrm{Pa}^{233}$, the protoactinium daughter of the $26-\mathrm{min}$. $\mathrm{Th}^{233}$ which is produced by neutron capture in thorium.

Hahn and Strassmann (O. Hahn and F. Strassmann, Naturwiss 28, 543 [1940]) have recently pointed out that the radioactivity of 25 days halflife assigned to $\mathrm{Pa}^{23} 3$, originally reported by Meitner, Strassmann and Hahn (I. Meitner, F. Strassmann and O. Hahn, Zeits f. Physik 109, 538 [1938]) to be the daughter of the 26 -minute $\operatorname{Th}^{23} 3$ produced by neutron capture in thorium, might actually be due to an isotope of zirconium. 
There is a 25-day zirconium formed in the fission of uranium by neutrons, and hence probably also in the fission of thorium by neutrons. Since Meitner, Strassmann and Hahn used zirconium as the carrier material for their radioactive substance, the question arises as to whether their 25day radioactivity from thorium plus neutrons might be due to an isotope of zirconium rather than protoactinium.

Gofman will begin by attempting to demonstrate whether this 25-day activity is indeed due to $\mathrm{Pa}^{233}$. Kennedy is collaborating with us in this research, making his main contribution in developing the counting equipment that will be required.

Tuesday, October 1, 1940

Frederick J. Leitz, Jr., a new graduate student, has elected to do his research with Kennedy and me. He will join us on our work on the separation of isotopes using our thermal diffusion (Clusius) column which we have set up outside the Rat House (Chemistry Annex). Leitz will share our office space in room 404 of the Rat House.

Thursday, October 10, 1940

Kennedy has placed into operation a thin-windowed (cellophane of thickness $3.5 \mathrm{mg} / \mathrm{cm}^{2}$ ) tubular proportional counter (identified as counter no. Z-15) for use in detecting alpha particles in the presence of beta radiation. Thus, all in all, we are now ready to look for an alphaemitting isotope of the new element with the atomic number 94 , such as might be produced in the deuteron bombardment of uranium, in which a new isotope of element 93 might be synthesized and decay by beta emission to such a detectable element 94 daughter.

Monday, November 4, 1940

After becoming familiar with the chemistry of thorium, protoactinium and uranium, gathering his required chemical equipment and learning about the various relevant counting apparatus, Gofman is getting underway with his $\mathrm{Pa}^{233}$ problem. He is working in room 307, Gilman Hall, the same room in which Wahl and Friedlander are situated.

Sunday, November 10, 1940

Gofman made his first bombardment of thorium nitrate today using neutrons produced by the bombardment of phosphorus with deuterons at the 60-inch cyclotron. (The phosphorus target was for another experiment 
and advantage was taken of the by-product neutrons.) The thorium nitrate was surrounded by paraffin and the bombardment took place from 10:45 a.m. to noon.

Gofman brought the thorium to room 307, dissolved it in water, then added $\mathrm{H}_{2} \mathrm{O}_{2}$ to precipitate insoluble thorium peroxyhydroxide which he washed with water and alcohol several times. He followed the decay of this using electroscope no. 3 with my help, and we found that the $\operatorname{Th}^{233}$ in this sample decays with a half-life of approximately 26 minutes as expected.

Sunday, November 24,1940

Gofman bombarded about $15 \mathrm{gm}$ of thorium nitrate encased in a large paraffin block for about an hour with the neutrons produced during the bombardment of phosphorus with 100 microamperes of deuterons in the 60inch cyclotron. The thorium was dissolved and precipitated from acid solution as the iodate after the addition of appropriate carriers for the fission products. After redissolving the thorium iodate in concentrated hydrochloric acid, successive precipitations of zirconium phosphate were performed. If the 25-day activity formed by the slowneutron bombardment of thorium is due to $\mathrm{Pa}^{233}$ resulting from the beta decay of $\mathrm{Th}^{233}$, then the successive zirconium phosphate precipitates (which coprecipitate the protoactinium) should have decreasing amounts of $\mathrm{Pa}^{233}$ beta radiation. Measurements using electroscope no. 3 do show that the intensities are less for the successive precipitates, consistent with the $\mathrm{Pa}^{233}$ growing from a parent of approximately 26 minutes halflife, presumably $\mathrm{Th}^{233}$. It remains to be demonstrated that beta radioactivity in the zirconium phosphate precipitates will decay with the expected 25-day half-life.

Wednesday, November 27,1940

Since our first bombardment of uranyl nitrate hexahydrate (UNH) with neutrons on August 30 to produce $93^{239}$ for its study and for the investigation of the chemical properties of element 93, we have made four more such bombardments. These UNH samples were irradiated with neutrons at the 60-inch cyclotron on September 11 (bombardment no. 93-2), October 4 (no. 93-3), November 11 (no. 93-4), and November 20 (no. 93-5). Wahl has processed all of these chemically in order to isolate and study the radiations and decay of $93^{239}$ to the point that he has become very proficient in the purification of radioactive element 93 preparatory to any possible subsequent bombardments that might include the production of additional isotopes of element 93 that will need to be distinguished from $93^{239}$. The purified $93^{239}$ fractions have decayed with the half-life of 2.3 days and absorption measurements of the radiations have shown that the beta particles have an upper energy limit of about $0.8 \mathrm{Mev}$ and the gamma rays are low in energy and low in relative intensity. 
Kennedy has placed into operation a new, more versatile, beta and gamma radiation detector. This consists of an air-filled ionization chamber connected to a General Electric FP-54 vacuum type amplifier (electrometer) and galvanometer with resistance (R) and shunt (S) arrangements to vary the sensitivity of the galvanometer reading ( $r$ ad in $\mathrm{mm}$ of deflection) over a wide range. We have four resistance settings-R2, R3, R4, R5-decreasing in magnitude and hence in sensitivity by a factor of about 10 for each change from R2 to R3 to R4 to R5 and five shunt settings-S1, S2, S3, S4, S5-decreasing the sensitivity by a factor of about $\sqrt{10}$ for each change 51 to 52 , etc. Thus at the most sensitive setting, R2SI, we have a sensitivity of about $10^{4} \mathrm{~mm}$ deflection per microcurie of beta radiation and at the least sensitive setting, R5S5, the sensitivity is $10^{3}(\sqrt{10})^{4}=10^{5}$ less; i.e., about $10^{-1} \mathrm{~mm}$ deflection per microcurie. The precise conversion factors must be established by making comparative measurements and the setup needs to be calibrated for each beta emitter. Below the aluminum window, about $6 \mathrm{~cm}$ in diameter and $0.2 \mathrm{mg} / \mathrm{cm}^{2}$ thickness, there is a sample holder arrangement consisting of a number of steps at increasing distances to allow radiation absorbers to be interposed when the sample is placed on a lower step. This setup is in room 303 .

Thursday, November 28,1940

Today I sent a handwritten letter to Edwin McMillan (Department of Physics, Massachusetts Institute of Technology) to indicate that wahl has perfected his techniques for isolating very pure samples of element 93. I knew that McMillan had used the new approach of bombarding uranium with $16 \mathrm{Mev}$ deuterons in the 60-inch cyclotron last summer and had obtained some evidence for the production of a second isotope of element 93 and some indications that this might have decayed to a daughter element 94 isotope. I suggested that since he has now left Berkeley to work at a war project at MIT and is therefore not in a position to continue this work, that we would be very glad to carry on in his absence as his collaborators.

Sunday, December 8, 1940

Gofman bombarded $50 \mathrm{gm}$ of thorium nitrate for several hours with the neutrons produced from the bombardment of phosphorus with deuterons at the 60-inch cyclotron in order to produce a stronger sample of the 25-day activity to be used to establish by chemical experiments that it is due to protoactinium. 
I received a handwritten letter dated December 8 from Edwin McMillan in which he informed me that he will not be back in Berkeley for some time and it would please him very much if I could continue the work on elements 93 and 94. He pointed out that he had made some earlier observations that indicate long lived alpha activity was building up in a sample of purified 93 isolated from uranium bombarded with $16 \mathrm{Mev}$ deuterons in the 60-inch cyclotron. He indicated that this new isotope of element 93 seemed to have a half-life of about one day and decayed with the emission of higher energy beta particles than those of $93^{239}$, which was also present prominently in the 93 fraction. He indicated that the alpha activity has some similarity in chemical properties to thorium.

Wednesday, December 11, 1940

Today wahl has completed another bombardment of $28 \mathrm{gm}$ of UNH, which started on Monday, December 2, and has proceeded intermittently since that date. The bombardment stopped at 6:00 p.m. He brought the bombarded UNH to his laboratory, room 307, Gilman Hall, and set it aside for subsequent chemistry (bombardment no. 93-6).

Friday, December 13, 1940

Wahl completed his chemistry on bombardment no. 93-6. He performed an oxidation-reduction cycle with $\mathrm{BrO}_{3}{ }^{-}$as the oxidizing agent, $\mathrm{SO}_{2}$ as the reducing agent, and $\mathrm{CeF}_{3}$ as the carrier for the $93^{239}$ which he labelled sample no. 93-6-1. Decay and absorption measurements will be made on this fraction. He also isolated a uranium fraction, purified by ether extraction and precipitation and reprecipitation of sodium uranyl acetate (labelled no. 93-6-2); decay and absorption measurements will also be made on this fraction.

Gofman began his chemical identification of the 25-day beta activity using material produced in last Sunday's bombardment. He plans to dissolve the thorium nitrate, precipitate zirconium phosphate to carry the 25-day activity, convert this to zirconium oxychloride, and then subject this to fractional crystallization from hydrochloric acid solution. Experiments by Grosse and Booth (A. V. Grosse and E. T. Booth, Phys. Rev. 57, 664 [1940]) have shown that in such a fractional crystallization the protoactinium concentrates in the mother liquor.

Saturday, December 14, 1940

Wahl made a target by decomposing $\mathrm{UO}_{2}\left(\mathrm{NO}_{3}\right)_{2} \cdot 6 \mathrm{H}_{2} \mathrm{O}$ (UNH) into $\mathrm{U}_{3} \mathrm{O}_{8}$ on a copper plate. Between the hours of $8: 00 \mathrm{p} . \mathrm{m}$. and 12 midnight the target, which was placed in a bell jar, was bombarded with $16 \mathrm{Mev}$ deuterons through a $2-\mathrm{mil}$ Al window for a total of approximately 230 microampere- 
hours on the 60-inch cyclotron (bombardment no. 93-7). From the resulting material we plan to isolate a chemical fraction containing 93 and study its radioactivity and also to look directly for element 94.

Sunday, December 15,1940

The target from last night's deuteron bombardment was brought to room 307, Gilman Hall, scraped with an ice pick 20 minutes after the end of bombardment, and the scrapings dissolved in hot concentrated $\mathrm{HNO}_{3}$. The resulting solution was green; forgetting about the $\mathrm{Cu}^{++}$from the target, Wahl thought $\mathrm{U}^{++++}$was present and attempted to oxidize the solution with several reagents. Finally, after reduction with $\mathrm{SO}_{2}$, a $\mathrm{CeF}_{3}$ precipitation was made, dissolved and reprecipitated. At about $2: 15$ a.m., one half of the $\mathrm{CeF}_{3}$ precipitate was mounted on cardboard to look for alpha particles (sample no. 93-7-1). The other half of the precipitate was put through an oxidation-reduction cycle to isolate elemate 93 and remove from it fission product activities; a final precipitate was filtered in a gooch crucible and mounted to study the beta and gamma radiation (sample no. 93-7-2). A sodium uranyl acetate fraction was isolated and labelled sample no. 93-7-3, and a zirconium phosphate fraction was isolated and labelled sample no. 93-7-4. We plan to follow the decay of these latter three samples. Absorption measurements taken on sample no. 93-7-2 using our ionization chamber FP-54 electrometer setup seem to indicate a new activity (in addition to $93^{239}$ ) in which the beta particles have an end point corresponding to an energy of about 1 Mev, substantially higher than that of $93^{239}$, and more intense gamma rays seem to be present.

Monday, December 16, 1940

Wahl made absorption measurements in $\mathrm{Al}$ on the $\mathrm{U}^{237}$ beta radiation from sample no. 93-6-2 (covered with cellophane) isolated last Friday, using our new FP-54 ionization chamber (which has an Al window of thickness $\left.0.2 \mathrm{mg} / \mathrm{cm}^{2}\right)$. The $U^{237}$ is produced in the $U^{238}(n, 2 n) U^{237}$ reaction. He found an end point of $100 \mathrm{mg} / \mathrm{cm}^{2}$, corresponding to an upper energy limit of less than $0.5 \mathrm{Mev}$ for the beta particles. The half thickness for absorption of the beta particles at the beginning of the absorption curve is about $6 \mathrm{mg} / \mathrm{cm}^{2}$, indicating the beta radiation is quite soft.

Tuesday, December 17,1940

Wahl's absorption measurements on the beta particles of the $93^{239}$ sample (no. 93-6-1, isolated last Friday), made using our FP-54 ionization chamber, show a half thickness at the beginning of the absorption curve of $8.7 \mathrm{mg}$ Al per sq. $\mathrm{cm}$. 
Gofman has completed his zirconium oxychloride experiments started last Friday and finds that the specific activities (activity per decigram $\mathrm{ZrO}_{2}$ ) of the first fractions are of the order of 5\% of the specific activity of the overall sample, showing an accumulation of the activity in the mother liquor as should be the case for protoactinium. This is further evidence that the 25-day activity is due to $\mathrm{Pa}^{233}$.

Thursday, December 19, 1940

Today Gofman extracted a purified uranium fraction through the precipitation and reprecipitation of sodium uranyl acetate from a solution of thorium nitrate made up of neutron-bombarded thorium. The purpose is to see whether there is any beta-emitting $\mathrm{U}^{233}$ daughter in the $\mathrm{Th}^{233} \rightarrow$ $\mathrm{Pa}^{233} \rightarrow \mathrm{U}^{233}$ decay sequence. He found no beta particles in the uranium fraction that could be attributed to $U^{233}$. It is, of course, to be expected that $\mathrm{U}^{233}$ will decay by the emission of alpha particles and we intend to conduct experiments to look for these.

\section{Monday, December 23, 1940}

The 93 sample (no. 93-7-2) measured without absorber has decayed with a half-life of 2.3 days, indicating that $93^{239}$ is the main component, as is to be expected since it is produced via both the $\mathrm{U}^{238}(\mathrm{~d}, \mathrm{n})$ and $\mathrm{U}^{238}$ $(d, p)$ reactions. However, when the decay of the sample was followed with various aluminum absorbers (for example, $450 \mathrm{mg} / \mathrm{cm}^{2}$ ) between the sample and detector, using both our new ionization chamber FP-54 electrometer and Lauritsen electroscope no. 3, the intensity decreases with a 2.1 to 2.2-day half-life, indicating that the more penetrating radiations are emitted by a second, slightly shorter-lived isotope of element 93. This new isotope of element 93 might be due to $93^{238}$, produced from the reaction $\mathrm{U}^{238}(\mathrm{~d}, 2 \mathrm{n}) 93^{238}$, or $93^{236}$, from $\mathrm{U}^{235}(\mathrm{~d}, \mathrm{n}) 93^{236}$, or $93^{235}$, from $\mathrm{U}^{235}$ $(d, 2 n) 93^{235 . .}$ we can not distinguish between these possibilities.

The sodium uranyl acetate sample (no. 93-7-3) shows only the 6.8-day activity associated with $\mathrm{U}^{237}$, and the zirconium phosphate sample (no. 93-7-4) shows only a 17-hour and a much longer decay period associated with $\mathrm{Zr}^{97}$ and $\mathrm{Zr}^{95}$, respectively, all of which are well known and have been observed previously from samples separated from $\mathrm{UO}_{2}\left(\mathrm{NO}_{3}\right) 2^{\circ} 6 \mathrm{H}_{2} \mathrm{O}$ that have been irradiated with neutrons.

Initially, the beta intensity of sample 93-7-1 was so large that our alpha proportional counter no. z-15 did not operate properly. Today, however, using another proportional counter built by Kennedy (z-18, similar to $\mathrm{z}-15)$, alpha particles from the sample were observed through the cellophane window of thickness $3.5 \mathrm{mg}$ per sq. $\mathrm{cm}$. We suspect that these might be due to element $94\left(94^{238}, 94^{236}\right.$ or $\left.94^{235}\right)$, the decay product of the new isotope of element 93. We plan to do tracer experiments with this alpha radioactivity to try to make observations on the chemical properties of this new element (if it is actually element 94). 
Initial experiments show that the alpha emitter does not distill as a volatile oxide with $\mathrm{OSO}_{4}$ from a hot solution of $\mathrm{HNO}_{3}$ and $\mathrm{NaBrO}_{3}$, as might be expected for a homolog of osmium, the prediction for element 94 according to the Periodic Table. It is coprecipitated by $\mathrm{LaF}_{3}$ from dilute acid, reducing solution (containing $\mathrm{SO}_{2}$ ) as is $93^{239}$.

In order to investigate another possible approach to the production of the new element with the atomic number 94 , we bombarded uranium oxide with helium ions (5.5 microampere-hours) between 5:00 and 7:00 p.m. in the 60-inch cyclotron, operated by MacKenzie, to see if any additional isotopes of elements 93 and 94 can be produced and identified. We designated this as bombardment no. 93-8. Wahl isolated an element 93 chemical fraction (no. 93-8-2) using the usual "oxidation-reduction cycle." Gerhart Friedlander measured the absorption in aluminum of the beta radiation from sample no. 93-8-2 using our Lauritsen electroscope no. 3. Using our ionization chamber FP-54 electrometer, I similarly measured the absorption of the beta radiations from the rare earth fraction (labelled no. 93-8-3) which might contain any element 94 isotopes. Attempts to detect long range alpha particles with a small portion of the original target material (93-8-i) spread thin and the element 93 (93-8-2) and rare earth (93-8-3) fractions, using our proportional counter no. z-18, gave negative results.

Tuesday, December 24, 1940

Continuing their chemical separations on bombardment no. 93-8, wahl isolated a uranium fraction (sodium uranyl acetate, labelled sample no. 93-8-8), and Friedlander a number of fission product fractions.

Wednesday, December 25,1940

Continuing his chemical separations on 93-8, wahl isolated purified element 93 (sample no. 93-8-9) and uranium (sample no. 93-8-10) fractions.

Friday, January 3, 1941

I had a discussion with Emilio Segre, a collaborator in many of my previous research problems. He has just returned from a visit to the East last month where he conferred in New York with Ernest Lawrence (who was also on a trip East) and Enrico Fermi who suggested that Segrè and I should try to produce enough $93^{239}$ and it unidentified daughter $94^{239}$, using the 60-inch cyclotron, to enable us to determine whether these isotopes undergo fission with slow neutrons. Segre also indicated that Fermi would like us to determine the yield of $93^{239}$ produced from the bombardment of uranium by the 60-inch cyclotron. Since segre and Fermi are both aliens, it isn't clear that I am authorized to reveal our recent 
results on the observation of a new isotope of element 93 that might be decaying to an alpha-emitting daughter (which would be an isotope of the new element 94 , such as $94^{238}, 94^{236}$ or $94^{235}$ ). However, since Ernest Lawrence has suggested that Segrè and I collaborate on the research to determine the fissionability of $93^{239}$ and $94^{239}$, I am proceeding on the assumption that I can inform Segrè and Fermi about our new isotope of element 93 and its alpha-emitting daughter (but not about the chemical properties of the latter).

Joe Kennedy and I have discussed, during the Christmas holidays, the prospects of producing and isolating enough $94^{239}$ to determine whether or not it undergoes fission with slow neutrons and have decided that it can be done.

Saturday, January 4, 1941

Kennedy has constructed a new type proportional counter based on information we have received from Dick Dodson of Caltech. This counter (no. $\mathrm{z}-20$ ) has a thin mica window $(3.6 \mathrm{mg}$ per sq. $\mathrm{cm}$ thickness). The design is of the bell jar type, about $5 \mathrm{~cm}$ long and $3 \mathrm{~cm}$ in diameter, with the mica window across the open end. The central wire has a small glass bead on the end and extends to within a few $\mathrm{mm}$ of the mica window.

Sunday, January 5, 1941

Today wahl attempted to oxidize the alpha activity (sample no. 93-7-1) with $\mathrm{BrO}_{3}{ }^{-}$in dilute acid solution to a form that is not coprecipitated by $\mathrm{LaF}_{3}$ but did not succeed in effecting such an oxidation; the alpha activity coprecipitates with $\mathrm{LaF}_{3}$ even in the presence of $\mathrm{BrO}_{3}{ }^{-}$. This then shows that the alpha activity is not due to element 93 because element 93 is oxidized to a higher oxidation state that is not coprecipitated by $\mathrm{LaF}_{3}$ under these same conditions. The alpha activity is similar in chemical properties to thorium, actinium, and the rare earth elements in this property of nonoxidation by $\mathrm{BrO}_{3}^{-}$.

Joe Kennedy is making measurements on the range of the alpha particles, using his proportional counter no. $z-20$ and sample no. 93-7-1. His preliminary results indicate the range to be about $3.9 \mathrm{~cm}$ in air equivalent (only slightly longer than corresponds to the thickness of the counter window), suggesting that the energy is similar to the alpha particles from Po 210 (5.3 Mev). 
Wahl performed experiments to determine whether our alpha activity (sample no. 93-7-1) plates out on copper metal and it was found that it does not.

Wednesday, January 8,1941

Wahl performed an experiment to see whether our alpha activity (sample no. 93-7-1) coprecipitates with CuS from dilute acid solution saturated with $\mathrm{H}_{2} \mathrm{~S}$. Since it does not, this experiment and that performed yesterday indicate that the alpha activity is not due to a noble metal homolog of osmium, as would be predicted from the Periodic Table.

Thursday, January 9, 1941

At 6:00 p.m. Wahl completed a two day intermittent bombardment of $50 \mathrm{gm}$ of UNH at the 60-inch cyclotron with neutrons produced by the irradiation of phosphorus (and other targets) with deuterons (bombardment no. 93-9). (The various targets- $\mathrm{P}, \mathrm{Be}, \mathrm{Fe}$, S-were being used in other experiments and thus advantage was taken of these sources of "free" neutrons.) He isolated an especially pure element 93 fraction using the usual "oxidation-reduction cycle" (i.e., with $\mathrm{BrO}_{3}^{-}$as oxidizing agent, $\mathrm{SO}_{2}$ as reducing agent, and $\mathrm{LaF}_{3}$ as carrier), but differing from previous experiments in that a large number of specific carriers for fission products were introduced to insure better separation from these contaminants. The purpose of this experiment is to calibrate our radiation detection instruments and establish the yield of our process for isolating element 93 as part of a program to determine the absolute yields of $93^{239}$ from neutron bombardments of uranium.

Segrè and I bombarded $10 \mathrm{gm}$ of UNH with the neutrons produced from the bombardment of a phosphorus target with the $16 \mathrm{Mev}$ deuterons ( 177 microampere-hours) in the 60-inch cyclotron (bombardment no. 93-10) as a first step in determining the yield and optimum conditions for producing $93^{239}$. The uranium was surrounded by paraffin in order to produce slow neutrons. The bombardment took place from noon to 6:00 p.m. and the reason for the phosphorus target was that this bombardment was for the primary purpose of producing $\mathrm{P}^{32}$ for other purposes and we are trying to determine whether we can utilize the neutrons produced as by-products.

Kennedy has constructed a new type Geiger-Mueller counter of the same design as our proportional counter no. z-20. This counter (which we designate as no. z-21), our first Geiger-Mueller counter of this type, is characterized by a stable thin window and convenient provision for counting samples on flat backing plates at fixed geometry. The design is of the bell jar type, about $5 \mathrm{~cm}$ long and $3 \mathrm{~cm}$ in diameter, with a thin mica window of $4.5 \mathrm{mg}$ per sq. cm thickness across the open end. The central wire, with a small glass bead on the end, extends to within a 
few $\mathrm{mm}$ of this thin mica window. Below the window is a sample holder arrangement consisting of a number of slots at increasing distances to allow radiation absorbers to be placed between the sample and the window when the sample is placed in a lower slot.

Friday, January 10, 1941

Today Segrè and I bombarded $5 \mathrm{gm}$ of UNH from 9:00 a.m. to 10:00 a.m. with the neutrons produced by the bombardment of a beryllium target with $16 \mathrm{Mev}$ deuterons (83 microampere-hours) in the 60-inch cyclotron (bombardment no. 93-11). The uranium was surrounded by paraffin in order to produce slow neutrons.

Saturday, January 11,1941

At about 1:00 a.m. Wahl used the pure element 93 fraction from the uranium-neutron bombardment no. 93-9 to make the first calibration of our ionization chamber connected to the FP-54 electrometer, and our Lauritsen electroscope no. 3, for the measurement of the intensity of beta radiation from $93^{239}$. He used our new thin-windowed $(4.5 \mathrm{mg}$ mica per sq. cm) Geiger-Mueller counter (no. z-21) to determine the absolute counting rate of a thin sample of $93^{239}$ (geometrical efficiency estimated by Kennedy and me as 32\%) and the method of aliquots to relate this low intensity sample (correcting for absorption in the counter window and intervening air) to the response of our ionization chamber FP-54 and our electroscope no. 3 to the beta radiation from samples of $93^{239}$ of higher beta intensity. The results indicate one microcurie of $93^{239}$ beta radiation, with the sample in the first slot (that is, on the first step), gives a deflection of $3.1 \times 10^{4} \mathrm{~mm}\left(1.5 \times 10^{4} \mathrm{~mm}\right.$ with the sample on the second step) using the ionization chamber FP-54 with the resistance-shunt setting $R 2 S 1$, and a rate of discharge of 3.1 divisions per second using electroscope no. 3. The samples were mounted on thin glass. These calibrations will also make it possible to make approximate determinations of the absolute intensities of radioactivity of other beta particle emitters as well.

Wahl then continued, using another portion of this pure element 93, to go through a "standard" oxidation-reduction procedure with a blank (unirradiated) sample of UNH, to which fission product carrier elements were added, in order to determine the chemical yield of $93^{239}$ obtained in the procedure. The plan is to use this "standard" procedure to isolate the element 93 from neutron bombardments nos. 93-10 and 93-11; the efficiency of the chemical procedure must be known in order to determine the yields of $93^{239}$.

Wahl then applied this "standard" procedure to the isolation of element 93 from bombardments no. 93-10 (UNH plus neutrons from phosphorus plus deuterons) and no. 93-11 (UNH plus neutrons from beryllium plus deuterons). 
In the afternoon Wahl, Segrè and I measured with the ionization chamber FP-54 electrometer the intensity of beta radiation from the element 93 fractions of bombardments no. 93-10 (UNH plus neutrons from phosphorus plus deuterons) and no. 93-11 (UNH plus neutrons from beryllium plus deuterons). These measurements, together with Wahl's ionization chamber calibration and chemical yield measurements, enable us to calculate the following yields for $93^{239}$ : from phosphorus plus deuteron neutrons, 0.0055 microcuries per microampere-hour per gram of UNH; and from beryllium plus deuteron neutrons, 0.15 microcuries per microamperehour per gram of UNH.

The production of microgram quantities of $94^{239}$, using neutrons furnished by the 60-inch cyclotron from deuteron-irradiated beryllium targets, is clearly feasible. Such a neutron bombardment of one kilogram of UNH at an integrated intensity of 1000 microampere-hours of deuterons should yield $150 \mathrm{millicuries}$ of $93^{239}$ corresponding to about 0.6 microgram of $94^{239}$ upon beta decay of the $93^{239}$. Of course, the yield will be somewhat less than this because of the poorer geometry of such a large sample of UNH and the reduced chemical yield of $93^{239}$ when it is completely separated and purified from so much uranium.

We also calculated the yield of total element 93 (a mixture of mostly $93^{239}$ with admixture of some of the new 2.2-day isotope) which we obtained in the direct bombardment of uranium with deuterons in the 60-inch cyclotron: 20 microcuries per microampere-hour. Thus it would require about 10,000 microampere-hours of bombardment to produce one microgram of $94^{239}$ by this route.

Gofman completed at midnight the bombardment of 35 gm of UNH with the neutrons produced by the bombardment of phosphorus with a total of 750 microampere-hours of deuterons at the 60-inch cyclotron. The purpose of the experiment is to isolate a sample of 17-hour zirconium, produced as a fission product, and subject it to a series of fractional crystallizations of zirconium oxychloride similar to those performed in experiments ending December 17, 1940. This will be a test of the method since the 17-hour zirconium should not, of course, undergo any separation from the zirconium oxychloride.

I sent a handwritten letter to Ed MCMillan at Massachusetts Institute of Technology describing to him our new results, namely the observation, after chemical separation of an element 93 fraction from deuteron-bombarded uranium, of a new isotope with a half-life slightly shorter $(2.2$ days) than that of $93^{239}$ (2.3 days), which is also present. I indicated that the 2.2-day activity decays by the emission of higher energy beta particles and more gamma rays than $93^{239}$ and hence is readily distinguishable from it. I also indicated that the new element 93 activity (which can be assigned to $93^{238}, 93^{236}$ or $93^{235}$ ) apparently decays to an alphaemitting daughter (range $3.9 \mathrm{~cm}$ in air) which may be element 94 and described our observations on its chemical properties.

I also sent an informal handwritten letter to Fermi telling him about our observation of the new element 93 beta activity and its 
apparent decay to an alpha-emitting element 94 daughter. I also passed on to him the yields of $93^{239}$ that were determined in our experiments of the last few days.

I sent a letter to Professor Walter wahl (Chemical Laboratory, University of Helsingfors, Helsingfors, Finland) informing him that I have read with interest the report on his discovery of a mass-line 237 published in the January 3, 1941 issued of Science magazine. I asked him for more information on the types of minerals which contain the element which has this mass-line, and on the localities from which these minerals were obtained. My purpose in writing him is to obtain information that might be useful in a search for transuranium elements in nature, a possible source of useful amounts of these elements.

Sunday, January 12,1941

I sent a handwritten letter to Philip H. Abelson, Secretary, Uranium Committee, Washington, D.C., informing him that I have obtained some $93^{239}$ yield data and have sent these and some other data, which I already had on hand, to Professor Fermi at the request of Dr. Segre (who has been asked by Professor Lawrence to help). I asked if he would notify Professor G. N. Lewis of the government's interest in this work. I also asked him if he could do something to enable me to keep my graduate student, Arthur Wahl, here; as a Reserve officer he is subject to call to duty. Wahl is an exceptionally good man, and will be of incalculable value to us in the future. I asked Abelson what he thought of my idea of making a search for element 93 in nature (as another project, which I outlined to him in a previous letter). Professor Lewis is very enthusiastic about this possibility. I informed Abelson that we now have here in Berkeley a laboratory complete with nearly every detection device for radiation (Geiger-Mueller counters, coincidence counting setup, proportional counters, ionization chamber with a FP-54 tube, and electroscopes) and also many experienced men: Kennedy, Wahl and several more if needed (I have in mind especially my first year graduate students, John W. Gofman and Spofford G. English). I noted that we are excellently equipped here to look for element 93 by inducing fission with 60-inch cyclotron neutrons; the mass-line reported by Walter wahl in the January 3, 1941 issue of science magazine might well be due to $93^{237}$, since element 93 might be expected to occur in such rare-earth containing ores. I suggested that we might put some such ores through a chemical procedure designed to isolate element 93.

Monday, January 13,1941

A second deuteron irradiation of about $2 \mathrm{gm}$ of $\mathrm{U}_{3} \mathrm{O}_{8}$ (bombardment no. 93-12) was made between 7:30 and 10:00 p.m. Conditions were similar to those of the first irradiation (bombardment no. 93-7, December 14, 1940), except that the uranium has been separated from its decay products 
$\left(\mathrm{Th}^{234}\right.$ and $\mathrm{Th}^{231}$ ) to reduce radioactive impurities, and the target received only 140 microampere-hours of $16 \mathrm{Mev}$ deuterons.

The bombarded target was brought into our laboratory, room 307, Gilman Hall, for chemical processing. Chemical separations were performed on this irradiated $\mathrm{U}_{3} \mathrm{O}_{8}$ by wahl. First a small fraction of the unseparated target material was mounted on cardboard (sample no. 93-12-1) mainly for the purpose of my following the overall decay of its beta particles (largely due to fission products) using our ionization chamber connected to the FP-54 electrometer; this started at 10:40 p.m.

The irradiated $\mathrm{U}_{3} \mathrm{O}_{8}$ was dissolved in concentrated $\mathrm{HNO}_{3}$, and after diluting and adding $\mathrm{Ba}$ (II), Sb(III), Te(IV), Mo(VI), Cd(II), Ce(III), $\mathrm{La}$ (III), and $\mathrm{Zr}$ (IV) carriers, $\mathrm{SO}_{2}$ was added and the solution boiled. After removing a white precipitate (possibly $\mathrm{BaSO}_{4}$ ) that formed, $\mathrm{HF}$ was added to the cooled solution, and the $\mathrm{LaF}_{3}-\mathrm{CeF}_{3}$ precipitate that formed was divided into thirds. One third was mounted on cardboard directly for counting (sample no. 93-12-2).

Tuesday, January 14, 1941

Continuing his chemical separation, wahl took a second third of the $\mathrm{LaF}_{3}-\mathrm{CeF}_{3}$ precipitated from dissolved uranium of our second deuteron irradiation (no. 93-12) and dissolved it in preparation for a new approach designed to determine the possible genetic relationship between the new beta emitting element 93 isotope $\left(93^{238}, 93^{236}\right.$ or $\left.93^{235}\right)$ and its alpha emitting daughter $\left(94^{238}, 94^{236}\right.$ or $\left.94^{235}\right)$. The plan is to "milk" the alpha emitting daughter from the solution containing the new element 93 isotope utilizing a chemical separation of the two elements. The idea is to oxidize the 93 to a fluoride soluble oxidation state under conditions where the daughter alpha radioactivity is not oxidized but remains in a fluoride insoluble state. As already demonstrated, $\mathrm{BrO}_{3}{ }^{-}$ meets these conditions. At about 1:00 a.m. he oxidized the 93 with $\mathrm{BrO}_{3}{ }^{-}$and then precipitated and separated $\mathrm{LaF}_{3}$ (sample no. 93-12-3), then added $0.5 \mathrm{mg}$ of $\mathrm{La}^{++}$. The intent is to allow this solution and precipitate to stand for 36 hours and then remove the $\mathrm{LaF}_{3}$ by centrifugation (sample 93-12-10).

Then the plan is to add another $0.5 \mathrm{mg} \mathrm{La}^{+++}$to the resulting solution, allow that suspension to stand for another 36 hours at which time the process is to be repeated (giving sample no. 93-12-11). After another 36 hours the process is to be repeated, giving sample no. 9312-12. The plan is to measure the alpha radioactivity of these to see if their intensities decrease in a manner that corresponds to the 2.1-2.2 day half-life of the element 93 parent. 
Joe Kennedy is constructing a new, more efficient alpha particle detector, a screened-window ionization chamber connected to a linear amplifier, which will be used for the measurement of these "milked" samples. The samples will be measured to determine their alpha radioactivity after the strong beta radioactivity has decayed to levels that will not interfere with the alpha particle intensity measurements. The screened-window makes it possible to detect low energy alpha particles not possible with the proportional counter.

Wahl took the third portion of the $\mathrm{IaF}_{3}-\mathrm{CeF}_{3}$ precipitate and carried it through two "oxidation-reduction cycles" with $\mathrm{BrO}_{3}{ }^{-}$as the oxidizing agent and $\mathrm{SO}_{2}$ as the reducing agent. In these cycles he precipitated $\mathrm{Ce}\left(\mathrm{IO}_{3}\right)_{4}$ and $\mathrm{Zr}_{3}\left(\mathrm{PO}_{4}\right)_{4}$ from the solution containing element 93 in its oxidized fluoride soluble state in order to more thoroughly remove fission product activity due to these and other elements. Again the beta radiation from the final purified element 93 sample (no. 93-12-4), isolated at about 9:00 a.m., was too large for the alpha proportional counter to operate properly, so the possible genetic relationship between the new alpha emitter and the new 2.1 to 2.2 -day element $93\left(93^{238}, 93^{236}\right.$ or $93^{235}$ ) could not be investigated by measuring directly the growth of alpha activity. However, this sample is very suitable for the measurement of the rate of decay and the absorption of the beta and gamma radiations from this latter isotope. In order to extend the results obtained from the element 93 samples produced in the previous deuteron irradiation, the plan is to make such measurements on this sample. Wahl made his first measurement of the absorption in aluminum of the beta particles from this sample using the ionization chamber FP-54 electrometer at about 9:00 a.m. He found an end point of $380 \mathrm{mg} / \mathrm{cm}^{2}$ of aluminum, corresponding to an upper energy limit of the beta particles of about $1 \mathrm{Mev}$.

I made measurements on the beta decay of samples 93-12-1 and 93-12-2, using the ionization chamber FP-54 electrometer, at about 1:00 a.m., 2:00 a.m. and 9:00 a.m. - these decays were consistent with those expected from fission products.

Gofman has finished his fractional crystallization of 17-hour zirconium with zirconium oxychloride and finds that the specific activities of all the fractions are the same as that of the overall sample, as is to be expected.

Gofman has followed the decay of the radioactivity in the zirconium phosphate precipitates extracted from the solution containing $\mathrm{Th}^{233}$ on November 24, 1940 and has found in each case a half-life of about 25 days, indicating that $\mathrm{Th}^{233}$ decays to a beta emitter of 25 -days halflife, which therefore must be $\mathrm{Pa}^{233}$.

His aluminum absorption measurements on the 25-day activity indicate an upper limit for the beta rays of about $0.4 \mathrm{Mev}$ and show the presence of gamma rays. 
We prepared a letter to the editor describing our work on the identification of the 25-day $\mathrm{Pa}^{233}$ entitled "Radioactive Isotopes of Protoactinium" under the authorship of G. T. Seaborg, J. W. Gofman and J. W. Kennedy. We are today sending this letter for publication in The Physical Review. In this letter we describe our experiments concerning the genetic relationship of the 25-day activity to its parent $\mathrm{Th}^{233}$, its chemical identification as protoactinium by the fractional crystallization of zirconium oxychloride, the absence of daughter $U^{23} 3$ beta activity, and the measurement of the upper energy limit of beta particles as $0.4 \mathrm{Mev}$.

I wrote a formal letter to Enrico Fermi at the Department of Physics, Columbia University, with a carbon copy to Lyman $J$. Briggs, the head of the Uranium Committee in Washington, D.C., informing him of our observation of the new beta-emitting element 93 isotope $\left(93^{238}, 93^{236}\right.$ or $93^{235}$ ), produced by the deuteron irradiation of uranium, and our tentative evidence for an alpha emitting daughter which might be due to the new element 94.

Segrè and I also wrote a formal letter to Fermi, with a copy to Lyman J. Briggs, informing him of our yields of $93^{239}$ from neutron bombardments of uranium. Expressed as microcuries per microampere-hour per gram of uranyl nitrate hexahydrate (in paraffin), we reported yields of 0.15 for a beryllium target and 0.0055 for a phosphorus target. We also reported our yield of total element 93 activity $\left(93^{239}\right.$ plus $93^{238}, 93^{236}$ or $93^{235}$ ) per microampere-hour of $16 \mathrm{Mev}$ deuterons (through $2 \mathrm{mils} \mathrm{Al}$ ) on uranium $\left(\mathrm{U}_{3} \mathrm{O}_{8}\right)$ as 20.0 microcuries. We indicated that the use of neutron activation is clearly preferable for the production of $93^{239}$, using a deuteron-bombarded beryllium target, and that we are now planning for the neutron bombardment of large amounts of uranium to produce maximal quantities of $93^{239}$ (and presumably $94^{239}$ ).

Saturday, January 18, 1941

I received a letter dated January 16, 1941 from Philip Abelson in reply to my letter to him of January 12, 1941. He indicated some concern about Segrè working with me on the fissionability problem, said that the final determination of his role should be left to Lawrence, and warned me that Fermi also is not fully cleared for all uranium information. He admonished me that all my results and data should be communicated only to Lyman Briggs, Chairman of the Uranium Committee. He indicated to me, in order of priority, that the most important problems are to determine whether elements 93 and 94 undergo fission with slow neutrons, what the cross sections are for this, whether they undergo fast neutron fission, and what are the fast neutron cross sections. He also indicated that he will investigate the possibility of doing something about Arthur Wahl's Reserve officer status and that Dr. Briggs will write to $G$. N. Lewis to enlist his cooperation in facilitating my work. He expressed approval of my idea to make a search for element 93 in nature and suggested that I prepare an official proposal requesting a grant of $\$ 1,000$ to support my preliminary work in this area. 
Measurements during the last few days with the proportional counter (z-20) on the now much less beta-active, purified element 93 sample (no. 93-12-4) from the second deuteron bombardment of uranium have shown the presence of alpha activity, presumably due to $94^{238}, 94^{236}$ or $94^{235}$.

I wrote to Phil Abelson in response to his letter dated January 16, 1941 indicating that I will communicate results only to Dr. Briggs. I also said that I believe that Dr. Lawrence favors the retention of Dr. Segrè on our investigations. I indicated that I will send Dr. Briggs an outline of my proposal to hunt for element $93\left(93^{237}\right)$ in nature within a few days.

I sent a handwritten letter to Ed McMillan bringing him up to date on our results of the second bombardment of uranium with deuterons. I indicated the evidence indicates that we have observed an isotope of element 94, but made the caveat that more experiments, especially chemical, are necessary in order to obtain final proof.

Tuesday, January 21,1941

Professor G. N. Lewis has written to Dr. Lyman Briggs stating that I will have his cooperation and that of the Berkeley Department of Chemistry in the work that I am undertaking for the uranium Committee. He is responding to a letter requesting such cooperation written to him by Dr. Briggs as a result of my request to Phil Abelson in my letter of January 12, 1941.

Wednesday, January 22,1941

Decay and absorption measurements on the various chemical fractions from our bombardment of uranium with helium ions (bombardment no. 93-8) on December 23, 1940, have during the intervening month led to the identification of a small amount of activity due to element 93, no observable $\mathrm{U}^{237}$ and no new heavy isotopes. A number of previously known fission products were identified, thus confirming that helium ion bombardments lead to fission of uranium, in agreement with earlier work of Fermi and segrè.

Thursday, January 23, 1941

I received a letter from Fermi dated January 21, 1941 in reply to my letter to him of January 15. On the basis of our information on the yields of $93^{239}$, he believes that it would be possible to determine the slow neutron fissionability of $94^{239}$ on a sample produced by the neutron bombardment of about $1 \mathrm{~kg}$ of UNH. He is quite concerned about whether we can extract a 94 sample which is sufficiently uranium-free from so 
much uranium (because the presence of fissions due to uranium would obscure the $94^{239}$ fissions) and therefore we should give consideration to producing the $94^{239}$ from deuteron bombardment of uranium, a method in which so much less uranium would be needed. He feels that if we can solve the chemical purification problem, the use of neutrons and $2 \mathrm{~kg}$ of UNH is the best approach and indicated that he is taking steps to have $5 \mathrm{~kg}$ of UNH shipped here to segrè. He said that should we have any difficulty in performing the fissionability tests on the isolated $94^{239}$, he would be glad to make the measurements on our final samples in his laboratory at Columbia University.

Friday, January 24, 1941

Segrè and I wrote to Fermi in response to his letter dated January 21,1941 to indicate our decision to use the neutron irradiation of large amounts of UNH rather than the direct deuteron irradiation of uranium for the production of our $94^{239}$ sample. We said we are developing an ether extraction apparatus with which we can separate most of the uranium from the $1-2 \mathrm{~kg}$ sample of UNH in such a manner that the product $94^{239}$ will be present at the aqueous chemical separation stage with no more uranium than would be present in the deuteron activated sample. We noted that the chemical treatment of the aqueous phase will involve us with some exposure to radiation because of the high level of fission product radioactivity; in order to obtain one microgram of $94^{239}$, approximately $1 / 4$ curie of $93^{239}$ will have to be handled.

Saturday, January 25,1941

Lawrence received a letter dated January 23 from Lyman Briggs commenting on my correspondence with Fermi (reference my letter of January 15, and his letter of January 21, 1941). Briggs said he has shown this correspondence to $\mathrm{Dr}$. Abelson and is passing on his comments. Abelson emphasizes that the best way of producing $93^{239}$ (and hence $94^{239}$ ) is by neutron bombardment. This is the same conclusion that we have come to and communicated to Fermi in our letter of yesterday.

I received a handwritten letter dated January 23, 1941 from McMillan commenting on my communications to him of our latest information on the new isotope of element 93 and its daughter alpha activity. He said that these results seem to be in agreement with the preliminary work that he did last summer. He said that Merle Tuve has talked to him about my coming East to join his group working on the uranium problem, but McMillan feels that I can be more valuable staying at Berkeley working with the use of the 60-inch cyclotron. 
Segrè received a letter from George B. Pegram, Columbia University, indicating that at the request of professor Fermi he is having Drakenfels and Company ship him 12 pounds of UNH for use in the experiments that are being undertaken in cooperation with Fermi.

Today we received delivery of a Lauritsen quartz-fiber electroscope that we ordered from the Fred $C$. Henson Company in Pasadena-this is our electroscope no. 5.

Tuesday, January 28,1941

I wrote to Phil Abelson giving an up-to-date report on the new element 93 isotope $\left(93^{238}, 93^{236}\right.$ or $\left.93^{235}\right)$ and its daughter alpha radioactivity which McMillan, Wahl, Kennedy and I have observed as the result of the bombardment of uranium with $16 \mathrm{Mev}$ deuterons in the Berkeley 60inch cyclotron. I indicated in this communication that the alpha particles seem to be due to the element 94 daughter of the 2-day 93 as indicated by the alpha particle growth. I said that this alpha-activity is chemically separable from uranium and 93. The chemical experiments so far indicate a similarity to thorium and the activity has not yet been separated from thorium. More chemical experiments definitely must be performed before it can be regarded as proved that the alpha-particles are due to an isotope of element 94 .

I wrote a letter to Lyman Briggs incorporating a proposal to the Uranium Committee for a search in nature for elements 93 and 94 in collaboration with Joseph $W$. Kennedy and Arthur $C$. Wahl. I outlined the general method of procedure based on our knowledge of chemical properties of elements 93 and 94 and mentioned especially the possibilities of finding the isotope $93^{237}$. I suggested that a budget of $\$ 1,000$ might be appropriate.

NOTE: The essential part of the communication to Abelson, excluding some introductory, background and personal material and the references to our uranium bombardment with alpha particles (bombardment no. 93-8), was given to Professor Gregory Breit in his capacity with Physical Review in Chicago on May 1, 1942 to be placed in the files of Physical Review for post-war publication. This paper was published as a letter to the editor of Physical Review entitled "Radioactive Element 94 from Deuterons on Uranium" by G. T. Seaborg, E. M. McMillan, J. W. Kennedy and A. C. Wahl, Phys. Rev. 69, 366 (1946). (April 1 and 15 issue.)

Friday, January 31, 1941

Today Segrè and I prepared our first large sample of UNH for neutron bombardment as a practice run to produce a large sample of $93^{239}$. We prepared a paraffin block $25 \mathrm{~cm}$ square and $25 \mathrm{~cm}$ deep housed in a wooden box with 3/4-inch sides. We placed $575 \mathrm{gm}$ of UNH in six soft (i.e., boron 
free) glass test tubes $(2.25 \mathrm{~cm}$ diameter and $17.5 \mathrm{~cm}$ long) in vertical holes in the paraffin. The UNH was a part of the 12 pounds which had been shipped to us by Drakenfels and Company at the direction of Pegram and Fermi.

Saturday, February 1, 1941

Our letter to the editor entitled "Radioactive Isotopes of Protoactinium" under the authorship of Seaborg, Gofman and Kennedy, appeared in today's issue of The Physical Review (Phys. Rev. 59, 321 [1941]). As a neighboring article in the same issue there is an article by Grosse, Booth and Dunning announcing similar results (Phys. Rev. 59, 322 [1941]).

Monday, February 3, 1941

Our paraffin block containing the $575 \mathrm{gm}$ UNH (surrounded by additional paraffin) was placed near the beryllium target of the 60-inch cyclotron and irradiated from $11: 00 \mathrm{a} . \mathrm{m}$. to noon with the neutrons produced by the bombardment of the beryllium with 40 microamperes of deuterons (total bombardment 40 microampere-hours).

We have had constructed by the Physics Department glass blower, Edward S. Luttropp, a Soxhlet apparatus for operation by remote control, capable of extracting into diethyl ether the uranium from uNH with the concurrent separation of the transmutation products (element 93, 94 and fission products) in the aqueous phase resulting from the water of crystallization of the UNH. This results in an initial separation of the element 93 (and any daughter 94) and fission products from the bulk of the uranium. This apparatus is situated in room 305 of the old chemistry Building and we will conduct our chemical separation procedures here. This room has recently been placed at our disposal and is very suitable because there is a large outdoor porch where we can carry out our evaporations, etc.

We used this neutron-bombarded UNH for a practice run with this ether extraction apparatus. At $3: 40 \mathrm{p} . \mathrm{m}$. we added the neutron-bombarded UNH from the six test tubes to 1.5 liters ether and operated the Soxhlet apparatus until about 6:00 p.m. The aqueous layer containing the element 93 and fission products had a volume of $46 \mathrm{cc}$.

Wednesday, February 5, 1941

Today we extracted the $93^{239}$ from the $46 \mathrm{cc}$ aqueous portion obtained in our extraction procedure of Monday by performing an oxidation-reduction cycle. We added $20 \mathrm{mg} \mathrm{La}$ and $20 \mathrm{mg}$ Ce carrier, precipitated these elements as fluorides by the addition of $\mathrm{HF}$, filtered the precipitate and 
dissolved it in acid, oxidized with $\mathrm{BrO}_{3}{ }^{-}$, by the addition of $\mathrm{HF}$ precipitated and removed $\mathrm{LaF}_{3}-\mathrm{CeF}_{3}$ (which contained the fission product rare earth activity), reduced the filtrate with $\mathrm{SO}_{2}$, added $3 \mathrm{mg} \mathrm{La}$ and $3 \mathrm{mg}$ $\mathrm{Ce}$ carrier, then added $\mathrm{HF}$ to precipitate $\mathrm{LaF}_{3}-\mathrm{CeF}_{3}$ (the element 93 fraction). Our measurements at 11:15 p.m., with the calibrated ionization chamber FP-54 electrometer setup in room 303 Gilman Hall, on the element 93 fraction show a total of 480 microcuries of $93^{239}$ when corrected for decay and chemical yields.

Thursday, February 6, 1941

At 10:00 a.m. we made another measurement of yesterday's $93^{239}$ sample and find this consistent with our yesterday's value of 480 microcuries. From our known yield of 0.15 microcuries of $93^{239}$ per gram of UNH per microampere-hour (established January 11) we can calculate the expected yield as $0.15 \times 575 \times 40=3400$ microcuries. Thus we find one-seventh of the expected yield which is consistent with the poor geometry of such a large sample and indicates that our practice run was generally successful.

Wahl completed his measurement of the decay of the new element 93 sample no. 93-12-4 (isolated from the second deuteron bombardment of uranium on the morning of January 14) made through absorbers to cut out as much as possible the beta radiation from $93^{239}$ which is also present in the sample. Measurements on the higher energy beta particles of the new isotope of element 93 were made through $225 \mathrm{mg} / \mathrm{cm}^{2}$ of aluminum and $556 \mathrm{mg} / \mathrm{cm}^{2}$ of aluminum. These measurements established the half-life of the new element 93 isotope $\left(93^{238}, 93^{236}\right.$ or $\left.93^{235}\right)$ as 2.1 days.

\section{Sunday, February 9, 1941}

Starting about noon today, using our new ionization chamber-linear amplifier outfit constructed by Kennedy, Wahl measured the intensities of the "milked" alpha radioactivities from the new 2.1 -day 93 isotope solution-the samples from the series of 36-hour milkings that he began early the morning of January 14. Sample 93-12-10 was "milked" the evening of January 15, 93-12-11 the morning of January 17, and 93-12-12 about noon of January 18. The beta radioactivity has subsided to levels that now make it possible to measure the intensities of the alpha radiation using Kennedy's new ionization chamber-linear amplifier outfit. When the samples were placed near the window of this ionization chamber, he found for sample 93-12-10 135 alpha counts per minute; for 93-12-11 200 alpha counts per minute; for 93-12-12 80 alpha counts per minute. Thus he succeeded in showing that the alpha radioactivity is growing from an isotope present in the mother solution, but neither the chemistry nor the alpha particle detection are sufficiently quantitative to make a definite assignment of the new 2.l-day isotope of element 93 as the parent of the alpha emitter. 
During the evening Wahl did some experiments with a part of the alpha radioactivity from bombardment $93-7$. He finds that this precipitates with $\mathrm{Th}\left(\mathrm{IO}_{3}\right)_{4}$ from acid solution under conditions where the rare earth fission products do not precipitate, thus effecting a separation of element 94 (if the alpha particles are due to 94) from the rare earth elements.

Wednesday, February 12, 1941

This afternoon Segrè and I bombarded $100 \mathrm{gm}$ of UNH, surrounded by paraffin, with the neutrons produced by irradiation of beryllium with deuterons (10 microampere-hours) at the 37-inch cyclotron. The purpose is to prepare $93^{239}$ for use in a second practice run using our ether extraction apparatus.

Thursday, February 13, 1941

This morning we ran the $100 \mathrm{gm}$ of UNH from yesterday's neutron bombardment through our ether extraction apparatus, then isolated the $93^{239}$ by performing an oxidation-reduction cycle essentially identical with that of February 5. Measurements on the ionization chamber FP-54 electrometer in room 303 indicated a satisfactory yield of $93^{239}$.

Saturday, February 15, 1941

In the afternoon we put the $93^{239}$ sample isolated on Thursday through another oxidation-reduction cycle and obtained a satisfactory yield.

Friday, February 21, 1941

Today we began the bombardment of $1 \mathrm{~kg}$ of thorium nitrate, imbedded in a box containing paraffin, with neutrons at the 60-inch cyclotron with the aim of preparing our largest samples of $\mathrm{pa}^{23}$ through an intensive bombardment.

Sunday, February 23, 1941

During the afternoon wahl performed experiments indicating that our alpha emitter (apparently $94^{238}, 94^{236}$ or $94^{235}$ ) can be precipitated from acid solution as a peroxy-hydrate using thorium as carrier.

During the last few days wahl has made attempts to oxidize our alpha emitter with $\mathrm{BrO}_{3}{ }^{-}$and also with $\mathrm{Ce}$ (IV), to a higher oxidation state which does not coprecipitate with $\mathrm{Th}\left(\mathrm{IO}_{3}\right)_{4}$ and was not able to do so. This 
indicates that stronger oxidizing agents than $\mathrm{BrO}_{3}^{-}$or $\mathrm{Ce}$ (IV) will be required to oxidize 94 to a higher iodate or fluoride soluble oxidation state, if this is possible at all.

With this in mind, following a suggestion of Professor Wendell $\mathrm{M}$. Latimer, Wahl investigated this evening the more powerful oxidizing agent, peroxydisulfate ion, $\mathrm{S}_{2} \mathrm{O}_{8}{ }^{-2}$, with silver ion catalyst. He used a sample of our alpha emitter which he had obtained as part of his chemical investigations with the material from our first deuteron bombardment (bombardment no. 93-7). This alpha emitter registered about 400 alpha counts per minute (above a Th background of 4 alpha counts per minute) on our new ionization chamber connected to a linear amplifier in room 303.

After dissolving the sample in concentrated acid, evaporating to a small volume, and diluting with water, he added a few hundred mg of $\mathrm{K}_{2} \mathrm{~S}_{2} \mathrm{O}_{8}$, about $10 \mathrm{mg} \mathrm{AgNO}_{3}$ and $0.5 \mathrm{mg} \mathrm{La}^{++}$carrier. After heating this solution and letting it stand for about half an hour, HF was added and the $\mathrm{LaF}_{3}$ precipitate (sample no. 93-7-33) was removed by centrifugation. Only about 10 alpha counts per minute were observed in the precipitate, indicating that our alpha emitter has been oxidized to a fluoride soluble oxidation state for the first time.

At $7: 15 \mathrm{p} . \mathrm{m}$. today Segrè and I began our bombardment of $1.2 \mathrm{~kg}$ of UNH for the purpose of producing a large sample of $94^{239}$ suitable for demonstrating its fissionability with slow neutrons. We placed the UNH in 12 soft glass test tubes $(2.25 \mathrm{~cm}$ diameter and $17.5 \mathrm{~cm}$ long) snugly imbedded in vertical holes in our paraffin block $(25 \mathrm{~cm}$ square and $25 \mathrm{~cm}$ deep) in a wooden box (with 3/4-inch sides), but in this case with the front side removed in order to place the UNH closer to the target. This was placed as close as possible to the beryllium target at the 60-inch cyclotron and the bombardment with $16 \mathrm{Mev}$ deuterons began at 7:15 p.m.

Monday, February 24, 1941

Continuing his investigations of yesterday past midnight, wahl heated the filtrate from sample no. $93-7-33$ on a hot plate in order to decompose the $\mathrm{S}_{2} \mathrm{O}_{8}{ }^{-2}$, then boiled the solution in order to further decompose any remaining oxidizing agent. After cooling, the residue was diluted with water, about $0.8 \mathrm{mg} \mathrm{La}^{+++}$was added, followed by the addition of $\mathrm{HF}$. The precipitate $\mathrm{LaF}_{3}$ (sample no. 93-7-34) was separated and found to contain most (a little more than 300 counts per minute) of the initial alpha activity, proving that the alpha emitter had indeed been oxidized to a higher oxidation state not carried by rare earth fluorides. This establishes that the alpha emitter is not a rare earth element or the rare earth-like element actinium; it also indicates that the alpha emitter is not an isotope of thorium, which cannot be oxidized and forms a slightly soluble fluoride, but it seems desirable to prove this essential point by repeating the experiment with $\mathrm{Th}^{234}$ tracer. Therefore, Wahl prepared a sample of beta-emitting $\mathrm{Th}^{234}\left(\mathrm{UX}_{1}\right)$ by coprecipitation with $\mathrm{LaF}_{3}$ from the aqueous phase of $45 \mathrm{gm}$ of ether-extracted UNH. 
The neutron bombardment of our $1.2 \mathrm{~kg}$ of UNH, which was continued throughout the night, was halted at 11:00 a.m. in order to make way for use of the 60-inch cyclotron by other experimenters; the integrated deuteron beam totalled 922 microampere-hours.

Wahl has followed the decay of the $U^{237}$ in sample no. 93-6-2 isolated on December 13, 1940, using our Lauritsen electroscope no. 3, and finds a half-life of 6.8 days.

Tuesday, February 25, 1941

Continuing past midnight his attempt to distinguish chemically between our alpha emitter $\left(94^{238}, 94^{236}\right.$ or $94^{235}$ ) and $T h$, wahl dissolved his $\mathrm{LaF}_{3}$ (containing the tracer $\mathrm{Th}^{234}$ ) and his sample no. 93-7-34 (containing about 300 counts per minute of our alpha emitter) in acid solution, diluted the solution with water and added approximately $200 \mathrm{mg} \mathrm{K}_{2} \mathrm{~S}_{2} \mathrm{O}_{8}$ and about $10 \mathrm{mg} \mathrm{AgNO}_{3}$. He then heated the solution, added $\mathrm{HF}$, cooled and removed the $\mathrm{LaF}_{3}$ precipitate (sample no. 93-7-36). He boiled the filtrate to destroy the $\mathrm{S}^{-} \mathrm{O}_{8}^{-2}$, added $0.5 \mathrm{mg} \mathrm{La}^{+++}$, then $\mathrm{HF}$ and separated the $\mathrm{LaF}_{3}$ precipitate (sample no. 93-7-37). Sample 93-7-37 contained a major portion of the alpha activity and very little of the $T^{234}$ beta activity, whereas sample 93-7-36 contained a substantial part of the $\mathrm{Th}^{234}$ beta activity and essentially none of the alpha activity. This indicates that the alpha activity, by oxidation with strong oxidizing agents to a fluoride soluble oxidation state, can be separated from Th which cannot similarly be oxidized to a fluoride soluble oxidation state.

With this final separation from Th, it has been demonstrated that our alpha activity can be separated from ail known elements and thus it is now clear that our alpha activity is due to the new element with the atomic number $94\left(94^{238}, 94^{236}\right.$ or $\left.94^{235}\right)$.

Friday, February 28, 1941

This evening Segrè and I put our $1.2 \mathrm{~kg}$ sample of UNH imbedded in paraffin back in its position near the beryllium target of the 60-inch cyclotron to resume its irradiation with neutrons.

Monday, March 3, 1941

The neutron bombardment of our $1.2 \mathrm{~kg}$ UNH sample was terminated at 6:00 a.m. this morning after it had received an integrated intensity of deuterons totaling 3368 microampere-hours. A little later, when segre and I came to work this morning, we transported this neutron irradiated UNH to room 305 old Chemistry Building to begin our long procedure for the isolation and purification of the produced $93^{239}$ which will be allowed 
to decay to $94^{239}$ to produce the sample that we will use to try to demonstrate the slow neutron fissionability of this isotope.

Wearing goggles for protection, we dissolved the $1.2 \mathrm{~kg}$ of uNH in 2 liters of diethyl ether in our soxhlet extractor. The remotely operated ether extraction procedure began at about 10:00 in the morning and continued until about $2: 30$ in the afternoon. We then removed the aqueous phase containing the $93^{239}$ (and daughter $94^{239}$ ) and fission products, placed it in a nickel coated platinum dish, reduced the volume by evaporation and added $10 \mathrm{mg} \mathrm{La}^{+++}$and $10 \mathrm{mg} \mathrm{Ce}$ as carriers. We added $\mathrm{HF}$ solution and precipitated the $\mathrm{LaF}_{3}-\mathrm{CeF}_{3}$. Since we have no centrifuge in this laboratory, we carried the suspension of precipitate in a centrifuge tube, placed in a lead beaker within a wooden box, to the chemistry room in Crocker Laboratory where we have a large centrifuge that we have used in much of our previous work. We wore lead impregnated gloves and balanced the centrifuge tubes and carried out the centrifugation behind a lead shield. After washing the precipitate with more $\mathrm{HF}$ and centrifuging again, we carried the precipitate (containing $93^{239}$, daughter $94^{239}$ and rare earth fission products) back to room 305 . We completed this much of our separation by 5:30 p.m.

We then washed the precipitate into a nickel coated platinum dish, dissolved it in acid and carried out the oxidation of element 93 with $\mathrm{BrO}_{3}{ }^{-}$in the usual manner. From this oxidized solution we precipitated $\mathrm{LaF}_{3}-\mathrm{CeF}_{3}$ by the addition of HF. Then, as before, we carried the centrifuge tube containing the suspended precipitate to the chemistry room in Crocker laboratory. Here the rare earth precipitate (containing any daughter $94^{239}$ and beta emitting rare earth fission products) was separated and placed in a marked bottle for storage. We then carried the solution containing the oxidized $93^{239}$ and also the bottle with the separated precipitate back to room 305, old Chemistry Building, using our carrying device. It was now about 10:00 p.m.

Also this afternoon wahl completed a bombardment (no. 93-13) started on Saturday of $107 \mathrm{gm}$ of UNH with neutrons from the irradiation of a beryllium target with deuterons at the 60-inch cyclotron. (Much of this bombardment was carried on concurrently with the bombardment that Segrè and I conducted for the long neutron irradiation of our $1.2 \mathrm{~kg} \mathrm{UNH}$.) He started the chemical process aimed toward the isolation of a sample of pure $93^{239}$ at 10:00 p.m., working as usual in room 307, Gilman Hall.

Tuesday, March 4, 1941

Continuing our work on the isolation and purification of the $93^{239}$ produced from the neutron bombardment of $1.2 \mathrm{~kg}$ of $\mathrm{UNH}$, we evaporated the solution, cooled it and added $\mathrm{SO}_{2}, 3 \mathrm{mg} \mathrm{La}{ }^{++}$and $3 \mathrm{mg} \mathrm{Ce}{ }^{+++}$, transferred the solution to a centrifuge tube and added $\mathrm{HF}$ to precipitate the $\mathrm{LaF}_{3}-$ $\mathrm{CeF}_{3}$ (containing the $93^{239}$ ). It was now 11:00 a.m. 
We carried this centrifuge tube with the suspended precipitate to the chemistry room in Crocker laboratory, separated the $\mathrm{LaF}_{3}-\mathrm{CeF}_{3}$ precipitate, washed the precipitate, and then carried the centrifuge tube containing this precipitate back to room 305, always with the same carrying arrangement. This precipitate contains the $93^{239}$ and the daughter $94^{239}$ that has grown in since the separation of the element 94 fraction from the $\mathrm{BrO}_{3}{ }^{-}$ -oxidized solution yesterday.

We then dissolved the precipitate in acid, added $\mathrm{SO}_{2}$ to insure that the $93^{239}$ and daughter $94^{239}$ were in the reduced fluoride insoluble oxidation state, added $\mathrm{HF}$, and precipitated the $\mathrm{LaF}_{3}-\mathrm{CeF}_{3}$ (still containing the $93^{239}$ and daughter $94^{239}$ ). This was the first of a planned six reprecipitations of the $\mathrm{LaF}_{3}-\mathrm{CeF}_{3}$ (containing the coprecipitated $93^{239}$, and $94^{239}$ continuously growing in) carried out to remove every last trace of uranium impurity. The centrifuge tube containing the suspended precipitate was carried in the same manner (in a lead beaker in a wooden box) to the chemistry room of crocker laboratory for centrifugation. We finished this step at approximately 4:00 p.m.

Following the identical procedure, a second reprecipitation was carried out which was completed at about 10:30 p.m.

Wahl completed the isolation of a sample of pure $93^{239}$ (no. 93-13-3). One of the prime uses planned for this sample is the calibration of our ionization chamber FP-54 electrometer setup in room 303 for the purpose of determining the absolute yield of our large sample of $93^{239}$ produced by the irradiation of the $1.2 \mathrm{~kg}$ UNH with neutrons. The calibration will entail making measurements at low geometry through an aluminum absorber in order to be able to cope with the high intensity expected from our large sample.

We concluded our bombardment of $1 \mathrm{~kg}$ of thorium nitrate, started on February 21, with neutrons at the 60-inch cyclotron. The material in a box filled with paraffin has been in various positions near the target (usually beryllium) bombarded with deuterons, sometimes in the best position and sometimes in a less advantageous position, depending on the competition for neutrons. Exposure corresponds to 9700 microampere-hours of deuterons.

Wednesday, March 5, 1941

This morning a third reprecipitation of our large sample of $93^{239}$ (and $94^{239}$ ) was completed at about noon, and a fourth at about 5:00 p.m.

In the afternoon wahl conducted measurements on steps $1,2,3,4$ and 5 and absorption measurements in aluminum with sample no. 93-13-3 for the calibration of our ionization chamber FP-54 electrometer setup in room 303, Gilman Hall. Combining these data with his calibration of January 11, he finds a deflection of $2.7 \times 10^{3} \mathrm{~mm}$ for $93^{239}$ beta radiation, with the sample on the 5th step at the resistance and shunt setting R2S1, and a diminution of the deflection by a factor of 4.0 upon the interposition of $18 \mathrm{mg}$ per $\mathrm{sq}$. $\mathrm{cm}$. of $\mathrm{Al}$ absorber. 
Continuing the purification of our large sample of $93^{239}$ (and 94239), our fifth and sixth reprecipitations were completed at about 12:30 p.m. The final precipitate was washed into a specially constructed circular platinum dish about $1.5 \mathrm{~cm}$ in diameter and 0.5 to $1.0 \mathrm{~cm}$ deep. The mixture was slowly evaporated to dryness, the sides of the dish were cut off, the sample covered with a thin layer of Duco cement, and the platinum dish was glued with Duco cement to a carboard backing, with completion of the operation at about 5:00 p.m. This is our final product containing our large sample of $93^{239}$ (and some daughter $94^{239}$ ) which we will allow to decay completely to daughter $94^{239}$ in order to test the slow neutron fissionability of the latter-we call this sample $A$.

In order to capture more completely the spirit of this rather extensive chemical separation procedure, carried out in a way to avoid as much as possible exposure to radiation, the original notebook entries are reproduced here. These were written before the experiment in the manner of instructions for guidance in the conduct of the rather complicated chemical procedure and were annotated as the separation progressed. Since the guidance instructions were followed faithfully, they serve as an actual account of how the chemical separation was carried out.

1. Put 2 liters of ether in flask, add the uranium nitrate to the extractor, and run the extractor for several hours. Wear goggles! (started boiling at about 10:00 a.m. and stopped at about 2:30 p.m., Monday, March 3, 1941.)

2. Boil down aqueous phase in nickel coated Pt dish outside on porch, cool and add $10 \mathrm{mg} \mathrm{La}$ and $10 \mathrm{mg} \mathrm{Ce}, 1 / 2 \mathrm{cc} 36 \mathrm{~N} . \mathrm{H}_{2} \mathrm{SO}_{4}$, transfer to wax tube and add enough $27 \mathrm{~N}$. $\mathrm{HF}$ to make soln. $6 \mathrm{~N}$. in $\mathrm{HF}$ ( $15 \mathrm{cc}$ of soln. $=65$ cc total).

3. Carry the centrifuge tube to Crocker Lab in $\mathrm{Pb}$ beaker in wooden box (also containing 2nd tube, wax beaker and wax bottle and $6 \mathrm{~N}$. HF) wearing $\mathrm{Pb}$ gloves. Balance behind $\mathrm{Pb}$ shield. Centrifuge for 20-30 minutes, pour liquid into beaker and then into wax bottle. Pour about $65 \mathrm{cc} 6 \mathrm{~N}$. HF into centrifuge tube and stir and centrifuge, add wash water to same wax bottle. Carry box to room 305 and put wax bottle into $\mathrm{Pb}$ cylinder on porch. (Completed this at $5: 30 \mathrm{p} . \mathrm{m}$. )

4. Wash precipitate into $\mathrm{Ni}$ coated $\mathrm{Pt}$ dish, using about $10 \mathrm{CC} \mathrm{H}_{2} \mathrm{O}$ and bakelite stirring rod, add $5 \mathrm{cc} 36 \mathrm{~N}$. $\mathrm{H}_{2} \mathrm{SO}_{4}$ and heat to fuming behind $\mathrm{Pb}$ shield in room 304, add about $50 \mathrm{CC} \mathrm{H}_{2} \mathrm{O}$ and 1 cC sat. $\mathrm{NaBrO}_{3}$ and heat and wait 10 minutes while warm and then cool soln. Transfer to 2nd wax tube, add $15 \mathrm{cc} 27 \mathrm{~N}$. HF.

5. Carry to Crocker $\mathrm{Lab}$ in $\mathrm{Pb}$ beaker in wooden box (also containing Ist wax tube), balance behind $\mathrm{Pb}$ and centrifuge. Carry box to room 305 and pour liquid into $\mathrm{Ni}$ coated $\mathrm{Pt}$ dish. Wash rare earth precipitate into wax bottle marked R.E. ppt. and put in $\mathrm{Pb}$ cylinder on porch. (Finished at about 10:00 p.m.)

6. Evaporate soln. to $\mathrm{H}_{2} \mathrm{SO}_{4}$ fumes in room 305 behind $\mathrm{Pb}$ shield. Finished at 12:00 p.m.) Add about $40 \mathrm{CC} \mathrm{H}_{2} \mathrm{O}$ and $\mathrm{cool}$ in ice bath add 10 
$\mathrm{cc}$ sat. $\mathrm{SO}_{2}$ in ice water and heat, add $3 \mathrm{mg}$ La and $3 \mathrm{mg} \mathrm{Ce}$. Transfer to 2nd wax centrifuge tube and add $18 \mathrm{CC} 27 \mathrm{~N}$. HF and stir. (Approximately 11:00 a.m. Tuesday, March 4, 1941.)

7. Carry to Crocker Lab (with lst tube and $6 \mathrm{~N}$. HF and wax bottle marked "lst 93 filtrate"), centrifuge, pour into wax bottle, wash in centrifuge with about 65 cC $6 \mathrm{~N}$. HF, pour into same wax bottle, carry to room 305.

8. Wash ppt. with about $10 \mathrm{CC} \mathrm{H}_{2} \mathrm{O}$ and new bakelite rod into old $\mathrm{PT}$ dish in old end of room 305 behind old PB shield on old electric heater. Add $5 \mathrm{cc} 36 \mathrm{~N}$. $\mathrm{H}_{2} \mathrm{SO}_{4}$, heat to fumes, add about $35 \mathrm{cc} \mathrm{H}_{2} \mathrm{O}, 1 \mathrm{cc}$ sat. $\mathrm{SO}_{2}$ in ice water, transfer to clean 3 rd centrifuge tube. Add 15 cc $26 \mathrm{~N}$. $\mathrm{HF}$ and stir.

9. Carry to Crocker Lab (with 2nd tube, $6 \mathrm{~N}$. HF and wax bottle marked "2nd 93 filtrate") and continue as in step 7. (Approximately 4:00 p.m. Tuesday, March 4, 1941.)

10. Repeat step 8 but use all new stuff at clean end and 4 th centrifuge tube.

11. Carry to Crocker Lab (with 3rd centrifuge tube, $6 \mathrm{~N}$. HF, and wax bottle marked "3rd 93 filtrate") and continue as in step 7. (Approx. 10:30 p.m. Tuesday, March 4, 1941.)

12. Repeat step 8.

13. Carry to Crocker Lab (with other centrifuge tube, $6 \mathrm{~N}$. HF and wax bottle marked "4th 93 filtrate") and continue as in step 7 . (Approx. 11:55 a.m. Wednesday, March 5, 1941.)

14. Repeat step 8.

15. Repeat step 13. (Approx. 5:00 p.m. Wednesday, March 5.)

16. Repeat step 8.

17. Repeat step 8, except use final bakelite tube. (Approx. 12:30 p.m. Thursday, March 6, 1941.)

18. Ppt. put into $\mathrm{Pt}$ dish $(1.5 \mathrm{~cm}$ diameter and $1 / 2$ to $1 \mathrm{~cm}$ deep), solution evap. off, sides snipped off, covered with thin layer Duco cement. (Approx. 5:00 p.m. Thursday, March 6, 1941.)

This is called Sample A.

We carried Sample A to room 303 Gilman Hall and measured the intensity of the beta particles from its $93^{239}$ on the calibrated ionization chamber FP-54 electrometer setup. The sample was placed on step 5 (the greatest distance from the ionization chamber of the sample holder arrangement) and the beta radiation was measured through $18 \mathrm{mg}$ of $\mathrm{Al} / \mathrm{cm}^{2}$ 
using the lowest sensitivity setting for the FP-54 electrometer. The efficiency of the ionization chamber FP-54 electrometer setup under these conditions was determined by wahl yesterday using his sample of $93^{239}$ (no. 93-13-3) isolated from bombardment 93-13 conducted on Monday. Measurements by Kennedy, Segrè and I show that as of 5:35 p.m. today Sample A gives a deflection of $384 \mathrm{~mm}$ (resistance and shunt setting R5S5). Using Wahl's calibration data, and using Kennedy's experimentally determined factor of $1.33 \times 10^{5}$ for conversion from resistance and shunt setting $\mathrm{R} 5 \mathrm{~S} 5$ to $\mathrm{R} 2 \mathrm{SI}$, we obtain as the intensity of $93^{239}$ beta radiation

$$
\frac{384 \times 1.33 \times 10^{5} \times 4.0}{2.7 \times 10^{3}}=76,000 \text { microcuries }=76 \text { millicuries. }
$$

Our plan is to follow the decay of the $93^{239}$ beta particles in sample A, using our ionization chamber FP-54 electrometer setup, and, if possible, the growth of the daughter $94^{239}$ alpha particles using our ionization chamber connected to a linear amplifier with magnetic field to bend out the $93^{239}$ beta-particles. This very useful new setup is just now being put into operation by Kennedy, who has joined Segrè and me on our $94^{239}$ project; his emphasis will be on the detection instrumentation aspects.

Friday, March 7, 1941

I wrote to Dr. Lyman J. Briggs, Chairman of the Uranium Committee in washington, describing our latest results on our alpha radioactivity formed in the $16 \mathrm{Mev}$ deuteron bombardment of uranium. I indicated that this radioactivity can be precipitated, in what is probably the +4 oxidation state, as a fluoride or iodate using a rare earth or thorium as carrier material, and as peroxy-hydrate or iodate using a rare earth or thorium as carrier material, and as peroxy-hydrate using thorium as carrier. I described our important result that in the presence of the extremely strong oxidizing persulfate ion $\left(\mathrm{S}_{2} \mathrm{O}_{8}{ }^{-2}\right)$ plus $\mathrm{Ag}$ ion as a catalyst, our alpha activity is oxidized to a higher oxidation state which does not precipitate as a fluoride. Thus, with persulfate ion we have found it possible to separate quantitatively this radioactivity from thorium, using the beta activity $\mathrm{UX}_{1}$ as an indicator for thorium. I said that these experiments make it extremely probable that our alpha radioactivity is due to an isotope of element 94.

In my letter to Briggs I also reminded him of the danger of losing Arthur $C$. Wahl through call to active service in his capacity as a Reserve Officer and indicated that Dr. Kennedy is now working with segrè and me on the problem of determining the fissionability of $94^{239}$. I asked whether I could pass on to Dr. Segrè my information on the chemical properties of element 94 .

I sent a handwritten letter to Phil Abelson in Washington telling him that I have written to Dr. Briggs to give him our latest information on the chemistry of element 94 and also alerting him to the danger of wahl having to go into the Army, and telling him that Kennedy is now working with Dr. Segrè and me on the $94^{239}$ problem. 
NOTE: The essential part of the communication to Dr. Lyman J. Briggs covering my description of our results and evidence for element 94 was given to Professor Gregory Breit in his capacity with Physical Review in Chicago on May 1, 1942 to be placed in the files of Physical Review for post-war publication. This was published as a letter to the editor of Physical Review entitled "Radioactive Element 94 from Deuterons on Unarium" by G. T. Seaborg, A. C. Wahl and J. W. Kennedy, Phys. Rev. 69, 367 (1946). (April 1 and 15 issue.)

Saturday, March 8,1941

Wahl has purified a small uranium fraction from our $1.2 \mathrm{~kg}$ of UNH (bombarded with neutrons) in order to make an estimate of the yield of $\mathrm{U}^{237}$ produced by the $\mathrm{U}^{238}(\mathrm{n}, 2 \mathrm{n}) \mathrm{U}^{237}$ reaction. Making measurements on our calibrated Lauritsen electroscope no. 3 , he finds a yield for $\mathrm{U}^{237}$ of 0.0045 microcuries per gram of uranium per microampere-hour of bombardment. Smaller samples bombarded with fast, rather than slow, neutrons, should give rise to larger yields. Our electroscope was not calibrated specifically for the beta radiation of $\mathrm{v}^{237}$, which gives rise to a relatively small source of error.

I wrote to McMillan to bring him up to date on our latest work on the chemical properties of element 94, emphasizing that we have succeeded in oxidizing 94 to a higher oxidation state which does not precipitate as a fluoride, thus making it possible to separate it quantitatively from thorium. I also indicated that I have sent this information on to $\mathrm{Dr}$. Briggs and that Kennedy is now working with me on the $94^{239}$ project.

Sunday March 9, 1941

At $4: 15$ p.m. Kennedy made another measurement through $18 \mathrm{mg} / \mathrm{Al}^{2}$, using the ionization chamber FP-54 electrometer setup, of the beta radiation from Sample A, with the sample on the 5th step. The deflection of $191 \mathrm{~mm}$ (resistance and shunt setting R5S5) corresponds to 38 millicuries using our calibration values.

Tuesday, March 11, 1941

A measurement by Kennedy of the beta radiation from Sample A at 10:20 p.m. under the same conditions as those of March 6 and March 9 shows a deflection of $107 \mathrm{~mm}$ (R5S5). He also re-determined the amount of absorption of the $93^{239}$ beta radiation in the $18 \mathrm{mg} \mathrm{Al} / \mathrm{cm}^{2}$ absorber and finds a factor of 2.75 rather than the factor of 4.0 found by wahl last Wednesday. Thus, the intensity of $93^{239}$ beta radiation in sample $A$ is now

$$
\frac{107 \times 1.33 \times 10^{5} \times 2.75}{2.7 \times 10^{3}}=14,000 \text { microcuries }=14 \text { millicuries }
$$


Using the new factor of $2.7^{5}$ (rather than 4.0) for absorption of the $93^{239}$ beta radiation in the $18 \mathrm{mg} \mathrm{Al} / \mathrm{cm}^{2}$, we can recalculate the intensity of $93^{239}$ in Sample $A$ as 52 millicuries at 5:35 p.m. on Thursday, March 6 and 26 Millicuries at $4: 15$ p.m. on Sunday, March 9.

Thursday, March 13, 1941

Today Wahl started his chemical separation procedure on our third bombardment (no. 93-14) of uranium with deuterons. At about 10:00 a.m. a tellurium-uranium target that has been bombarded since Tuesday with 16 Mev deuterons for 2800 microampere-hours by Joe Hamilton to produce $I^{131}$ for medical purposes, was milled, and the metal chips were placed in a distilling flask. Concentrated nitric acid was added, and the solution boiled. Iodide ion carrier and more $\mathrm{HNO}_{3}$ were added, and $\mathrm{I}_{2}$ was distilled, trapped, and taken by Hamilton. The radiation level was high, and except when Hamilton needed help with an operation, wahl and I stood across the room from the target material being processed.

The residue from the distillation was transferred to a large beaker with water and heated about two hours, during which time $\mathrm{HNO}_{3}$ was added several times in an effort to get all solid material into solution. After half or more of the highly radioactive solution had been accidently spilled on the floor and mopped up with paper towels (which were treated with $\mathrm{HNO}_{3}$ and $\mathrm{H}_{2} \mathrm{SO}_{4}$ for potential recovery), the remaining (unspilled) suspension was centrifuged, and element 93 separated from the solution after two oxidation-reduction cycles with $\mathrm{BrO}_{3}{ }^{-}$as oxidizing agent and $\mathrm{SO}_{2}$ as reducing agent, using $\mathrm{CeF}_{3}$ as carrier (sample no. 93-14-1). Another portion containing 93 was isolated in a $\mathrm{CeF}_{3}$ precipitate and mounted on Pt separately for radioactivity measurements (sample no 93-14-2).

Using the ionization chamber FP-54 electrometer setup, the plan is to follow the decay of sample no. 93-14-2 with and without aluminum and lead absorbers in order to establish a better value for the half-life of the new element $93\left(93^{238}, 93^{236}\right.$ or $\left.93^{235}\right)$ activity. It is also planned to use this sample to follow the growth of daughter 94 alpha activity in order to establish whether it is growing at a rate consistent with a halflife of about 2.1 days for the decay of its parent. It is now possible to start these alpha growth measurements immediately because we have Kennedy's ionization chamber-linear amplifier setup in which the ionization chamber is separated from the sample by a magnetic field which deflects the intense beta radiation.

Saturday, March 15, 1941

Continuing his chemical separations of Thursday, wahl dissolved the first rare earth precipitate from the $\mathrm{BrO}_{3}{ }^{-}$solution, which contains the 94 daughter alpha activity, and separated the 94 from the rare earth fission products using an oxidation-reduction cycle with $\mathrm{S}_{2} \mathrm{O}_{8}^{-2}$ plus 
$\mathrm{Ag}^{+2}$ as oxidizing agent and $\mathrm{SO}_{2}$ as reducing agent and $0.2 \mathrm{mg} \mathrm{Ce}{ }^{+3}$, precipitated as the fluoride, as carrier (sample no. 93-14-10). This precipitate was dissolved in $\mathrm{H}_{2} \mathrm{SO}_{4}$ and diluted to $25 \mathrm{cc}$ (designated sample no. 93-14-K) to serve as a source of 94 tracer. A 1 cc portion of this solution was taken and its 94 content coprecipitated with $0.2 \mathrm{mg} \mathrm{Ce}{ }^{+3}$ as the fluoride (sample no. 93-14-11); this sample will be useful as a "standard" source of $94\left(94^{238}, 94^{236}\right.$ or $\left.94^{235}\right)$ alpha particles.

Sunday, March 16, 1941

Wahl made measurements on sample no. 93-14-2 (a 93-containing $\mathrm{CeF}_{3}$ precipitate obtained from our third uranium deuteron bombardment last Thursday) to test whether positrons are emitted. With $556 \mathrm{mg} \mathrm{Al} / \mathrm{cm}^{2}$ between the sample and detector to absorb all beta particles, a block of paraffin was placed back of the sample to supply material in which positrons, if present, would be annihilated with emission of the $511 \mathrm{kev}$ gamma radiation. No significant increase in gamma activity was observed (approximately 3\%), indicating few, if any, of the beta particles are positrons.

He also made aluminum and lead absorption measurements, using our ionization chamber FP-54 setup, on sample no. 93-14-1. The aluminum measurments show an end point of $380 \mathrm{mg}$ per sq. $\mathrm{cm}$ corresponding to an upper energy limit for the beta particles of $1.0 \mathrm{Mev}$ according to Feather's rule, $R\left(\mathrm{gm} \mathrm{Al} / \mathrm{cm}^{2}\right)=0.543 \mathrm{Mev}-0.160$; this must be assigned to our new element 93 activity because the beta radiation from the $93^{239}$, also present in the sample, is completely absorbed by some $260 \mathrm{mg}$ per sq. $\mathrm{cm}$ of $\mathrm{Al}$. The lead measurements show a half thickness for the high energy component of $9.5 \mathrm{gm}$ of lead, corresponding to a gamma-ray energy of about $1.0 \mathrm{Mev}$; this also must be assigned to our new element 93 activity because the gamma radiation from the 2.3 -day $93^{239}$ is completely absorbed by much less lead.

I sent a handwritten letter to John $w$. Hamaker, a senior undergraduate student at UCLA who has been admitted to graduate work and awarded a Teaching Assistantship at Berkeley. In view of the fact that he has some experience in analytical microchemistry, having worked at UCLA with Professor W. R. Crowell (Professor of Analytical Chemistry at UCLA), I indicated that he might want to do his graduate work at Berkeley with me since I am carrying on a research program in which his experience would be valuable. (I did undergraduate research and helped crowell teach his quantitative analysis classes when I was a student at UCLA.)

Monday, March 17, 1941

Kennedy finds a deflection with the ionization chamber FP-54 setup corresponding to 3.7 millicuries for sample $A$ when the calibration factors are applied. 
Wahl completed his measurement of the growth of alpha particles in sample no. 93-14-2 using the ionization chamber-linear amplifier-magnetic field setup and finds that this growth is consistent with a half-life of 2.1 days for the beta-emitting parent. His measurement, begun on March 13 and 14, of the decay of the beta radiation through aluminum and lead absorbers indicates that our new $93\left(93^{238}, 93^{236}\right.$ or $\left.93^{235}\right)$ activity has a half-1ife of $2.1 \pm 0.1$ days.

Wednesday, March 26, 1941

Kennedy measured Sample A at 10:00 a.m. as 0.45 millicuries.

Friday, March 28, 1941

This morning Kennedy, Segrè and I made our first test for the fissionability of $94^{239}$ using sample $A$. The beta decay of $93^{239}$ since the isolation of Sample A on March 6 has been consistent with a half-life of 2.3 days and the $93^{239}$ has decayed essentially completely to $94^{239}$. The remaining beta activity due largely to $93^{239}$, about 0.25 millicuries, is too small to interfere with the measurements and the alpha activity in Sample A, as measured with our ionization chamber-linear amplifiermagnetic field setup at relatively low geometry (a little over 1\%), is 240 alpha counts per minute. A few measurements of the growth of $94^{239}$ alpha activity in Sample $A$ have been made by Kennedy, since its isolation on March 6, using our ionization chamber-linear amplifier-magnetic field setup, and these are consistent with the 2.3-day half-life of the betaemitting parent $93^{239}$.

Kennedy has constructed during the last few weeks a portable ionization chamber and linear amplifier suitable for detecting fission pulses when a sample is placed near the screened window of the ionization chamber and irradiated with neutrons. Sample A (estimated to contain 0.25 micrograms of $94^{239}$ ) was placed near the screened window of the ionization chamber imbedded in paraffin near the beryllium target of the 37-inch cyclotron. The neutrons produced by the irradiation of the beryllium target with $8 \mathrm{Mev}$ deuterons give a fission rate of 1 count per minute per microampere. When the ionization chamber is surrounded by a cadmium shield, the fission rate drops to essentially zero, indicating that the fissions are being induced by slow neutrons. A sample of natural uranium (very roughly estimated to contain about 0.5 micrograms of $\mathrm{U}^{235}$ ) similarly placed gives 11 slow neutron induced fission counts per minute per microampere.

This gives strong indications that $94^{239}$ undergoes fission with slow neutrons. A very rough estimate of the cross section is $1 / 11 \times 0.5 / 0.25=$ $\overline{1 / 5}$ that of $\mathrm{U}^{235}$. Unfortunately, Sample $\mathrm{A}$ is too thick (estimated 4.8 $\mathrm{mg} / \mathrm{cm}^{2}$ thickness plus Duco cement cover) to permit any accurate determination of the slow neutron fission cross section, or the alpha half-life, of $94^{239}$, due to the appreciable absorption of the fission fragments and alpha particles. 
During the last month Gofman has carried out a number of experiments to develop his method for the extraction of $\mathrm{Pa}^{233}$ from the bombardment of our $1 \mathrm{~kg}$ of thorium nitrate with neutrons at the 60-inch cyclotron between February 21 and March 4. Starting today he will make his initial extraction by precipitating zirconium phosphate to carry the $\mathrm{Pa}^{233}$ and then will separate the $\mathrm{Pa}^{233}$ from the zirconium by precipitating it with lanthanum oxalate under conditions which leave more than $90 \%$ of the zirconium in solution, presumably as an oxalate complex.

Today I received a handwritten letter from McMillan dated March 26, 1941 responding to my letter to him of March 8, 1941. He expressed delight with our latest results on element 94 and reviewed some of his preliminary evidence for rare earth-like chemical properties of the alpha radioactivity that he and Abelson investigated last summer as a possible daughter of $93^{239}$; it was of such low intensity that they thought it might be due to some kind of contamination. He also said that he has talked to Lawrence during his recent visit to MIT and Lawrence is working on obtaining permission for me to talk to segrè about the chemical properties of 94 .

Saturday, March 29, 1941

Wahl made absorption measurements, by varying the pressure of air in a silver coated (inside) flask with the central electrode connected to our FP54 electrometer (an adaptation of the method of Rutherford), on the range of the alpha particles in sample no. $93-14-2\left(94^{238}, 94^{236}\right.$ or $94^{235}$ from the third uranium-deuteron bombardment of March 11-13). He finds a range equivalent to about $3.9 \mathrm{~cm}$ of air, corresponding to about $5.3 \mathrm{Mev}$.

\section{Monday, March 31, 1941}

Today, from 5:00 to 9:00 p.m., Wahl bombarded $700 \mathrm{gm}$ (bombardment no. 93-15) of purified UNH with the fast neutrons produced by the bombardment of beryllium with 500 microampere-hours of deuterons at the 60inch cyclotron. The UNH was placed directly behind the beryllium target with no intervening paraffin in order to measure the yield of $\mathrm{U}^{237}$ under the best conditions for large scale production. This UNH was purified last Thursday by dissolving a larger sample of UNH in ether, extracting the water soluble impurities in the aqueous phase, then recovering the uranium from the ether phase and recrystallizing it from water; this procedure removes to a high degree the impurities present in the original UNH as it was purchased.

I wrote to Dr. Briggs today enclosing copies of letters received from the War Department by Professors Gilbert N. Lewis and Ernest 0 . Lawrence which are ambiguous about wahl's deferment status. I suggested that he might get in touch with The Honorable Robert P. Patterson, Under-Secretary of War, in order to obtain wahl's deferment from 
active military service so that he can continue his role in our program of research on the transuranium elements.

Tuesday, April 1, 1941

This afternoon we continued our measurements on the slow neutron fissionability of $94^{239}$ using our Sample A. In order to get a better idea of the cross section, we also made measurements for comparison with a new electroplated sample of natural uranium (U Standard No. 3) which has a weight of about $0.35 \mathrm{mg}$ of uranium (2.5 micrograms $\left.\mathrm{U}^{235}\right)$. In this comparison of the slow neutron fission counts of $94^{239}(0.86$ per minute per microampere of deuterons) with that of $U^{235}(12.4)$, the cross section of $94^{239}$ seems to be about 708 of that of $U^{235}$. However, we know that our Sample $A$ is too thick, containing as it does $3 \mathrm{mg} \mathrm{La}$ and $3 \mathrm{mg} \mathrm{Ce}$ as the fluorides and the estimates of the weights of the $94^{239}$ and $U^{235}$ are very rough.

Having followed the decay since March 14, 1941 of 93 sample no. 93-14-1 through aluminum absorbers of thickness $255 \mathrm{mg}$ and $450 \mathrm{mg}$ per sq. cm, Wahl has observed a half-life of about 2.1-2.2 days. Since the aluminum screens out the beta particles of the $93^{239}$ present in this sample, which was produced by deuteron bombardment of uranium, this again indicates clearly that the half-life of our new 93 isotope is less than that of the 2.3 day $93^{239}$.

Wednesday, April 2, 1941

Wahl dissolved the rather large sample of $700 \mathrm{gm}$ of uNH bombarded Monday (bombardment no. 93-15) in ether, separated the aqueous phase, washed the uranium-containing ether with water several times, extracted some of the UNH thus purified from fission products (and elements 93 and 94) back into water, mounted on cardboard with cellophane covering 100 $\mathrm{mg}$ of the finely ground purified UNH (sample no. 93-15-1) and $16 \mathrm{mg}$ of the finely ground purified UNH (sample no. 93-15-2) to measure the intensity and follow the decay of their contained $U^{237}$ on Lauritsen electroscope no. 5 .

Continuing our measurements on Sample A at the 37-inch cyclotron which were started Friday, comparing the neutron induced fission rate of Sample A and U Standard No. 3, Kennedy, Segrè and I obtained essentially the same results as yesterday. We are convinced that the 94239 in this

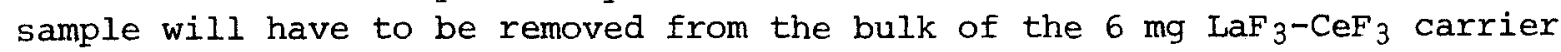
in order to obtain a thinner sample in which a much larger fraction of the fission fragments can be detected. 
The intensities of the $\mathrm{U}^{237}$ beta radiation in sample nos. 93-15-1 and 93-15-2 at 2:15 a.m., measured on electroscope no. 5, step 1, are 0.21 and 0.054 divisions per second (which corresponds to 0.26 and 0.069 divisions per second at end of bombardment). Since the sensitivity of the electroscope for $\mathrm{U}^{237}$ beta radiation is not known, it is not yet possible to calculate the absolute intensity of $\mathrm{U}^{237}$ beta radiation in these two aliquots of $\mathrm{UNH}$ (out of the total $700 \mathrm{gm}$ ). We wish to make an accurate determination of the large scale yield of $\mathrm{U}^{237}$, but will defer calculation until our Lauritsen electroscope no. 5 has been calibrated specifically for $\mathrm{U}^{237}$ beta radiation.

Continuing his work on bombardment no. 93-15, wahl turned to the initial aqueous phase formed from the water of hydration, and subjected it to two oxidation-reduction cycles using $\mathrm{BrO}_{3}^{-}$as oxidizing agent, $\mathrm{SO}_{2}$ as reducing agent, and rare earth fluoride as carrier. A number of carriers for fission products were added in order that these activities might be removed as completely as possible. The final sample of $93^{239}$ (no. 93-15-3) was mounted on Pt backing material with the intention of making measurements on the $93^{239}$ beta radiation and also the $94^{239}$ alpha particles that will grow into the sample. The plan is to measure the growth of alpha particles using our new ionization chamber-linear amplifier-magnetic field setup.

Saturday, April 5, 1941

Using our ionization chamber FP-54 setup, Wahl made absorption measurements in aluminum of the beta radiation of $93^{239}$ using sample no. 93-15-3 isolated on Thursday. He found a range of $260 \mathrm{mg} \mathrm{Al} / \mathrm{cm}^{2}$ which corresponds to an upper energy limit of $0.78 \mathrm{Mev}$ according to Feather's rule, $\mathrm{R}\left(\mathrm{gm} \mathrm{Al} / \mathrm{cm}^{2}\right)=0.543 \mathrm{E}$ (MEV) - 0.160 . His lead absorption measurements also show gamma rays over the energy range of $0.1-0.3 \mathrm{Mev}$.

Monday, April 7, 1941

Today an official letter was sent by the University of California Administration to the office of the Assistant secretary of War, Washington, D.C. requesting the transfer of 2nd Lt. Arthur C. Wahl, Jr. to the war Department Reserve Pool.

Thursday, April 10, 1941

Gofman has completed the extraction, which he started on March 28, of $\mathrm{Pa}^{233}$ from the $1 \mathrm{~kg}$ of neutron-bombarded thorium nitrate. He dissolved the thorium nitrate in water, precipitated zirconium phosphate to carry the $\mathrm{Pa}^{23} 3$ dissolved this in HF, converted to the hydroxide, dissolved this in $\mathrm{HCl}$, 
and precipitated lanthanum oxalate which carried the $\mathrm{Pa}^{233}$ while leaving the zirconium in solution. He had to handle numerous fractions and combine precipitates in order to carry out in practice this separation and concentration procedure, which is so simple in principle but difficult in execution; a number of cycles were carried out. He wound up with the final $\mathrm{Pa}^{233}$ in several fractions which he mounted in order to test for the growth of daughter $\mathrm{U}^{233}$ alpha particles. Two of these fractions have intensities of $\mathrm{Pa}^{233}$ beta radiation of 27 and 40 microcuries, as measured with our FP-54 ionization chamber and using Wahl's calibration of January 11, 1941. Wahl's calibration, $3.1 \times 10^{4} \mathrm{~mm}$ galvanometer deflection per microcurie, with the standard resistance and shunt setting R2Sl, is for $93^{239}$ beta radiation and hence its application to $\mathrm{Pa}^{233}$ beta radiation can give only approximate results, but these are sufficient for our purposes. Gofman will attempt to measure the growth of alpha particles in these $\mathrm{Pa}^{233}$ samples using our screen-windowed ionization chamber setup.

Thursday, April 17, 1941

Kennedy and Segrè made another comparison of the slow-neutron-induced fission rate of Sample $A$ and $U$ Standard No. 3 at the 37-inch cyclotron using an improved paraffin arrangement and an improved ionization chamber and linear amplifier built by Kennedy during the last couple of weeks. The counting rates are now 5 times higher but the results are not much different than those of our experiments of April 1 and 2, confirming that we are definitely observing the fission of $94^{239}$ with slow neutrons with a cross section comparable to that of $\mathrm{U}^{235}$.

Friday, April 18, 1941

The $U^{237}$ sample isolated by Wahl on April 2 (no. 93-15-2) is decaying with a half-life of 6.8 days, indicating that it is radioactively pure.

Wednesday, April 23, 1941

A small but discernible quantity of alpha activity has grown into the beta-emitting $\mathrm{Pa}^{233}$ samples isolated by Gofman on April 10. Measurements with our screen-windowed ionization chamber indicate that the 40 microcurie sample shows an alpha counting rate of 2 per minute, and our 27 microcurie sample shows an alpha counting rate of 1.2 per minute. Assuming that the alpha particles are due to daughter $\mathrm{U}^{23}$ (which needs to be proved), taking into account that the 25 -day $\mathrm{Pa}^{233}$ has decayed for 13 days, and correcting for the geometrical efficiency of the alpha counting ionization chamber, we can calculate the half-life for the daughter $\mathrm{u}^{233}$, as an average of the two determinations, to be of the order of $1 \times 10^{5}$ years. 
This, of course, is a very rough determination, and should perhaps be regarded as a lower limit. However, it may indicate that we should be able to produce, with more intense neutron bombardments of larger amounts of thorium, sufficient $\mathrm{U}^{233}$ to not only determine its alpha half-life, but also its cross section for fission with slow neutrons. Of special importance is our demonstration through these results that $\mathrm{U}^{233}$ is sufficiently long-lived to be a practical source of nuclear energy should it be found to be fissionable with slow neutrons and should methods for its large scale production be developed.

Friday, April 25, 1941

At 2:00 p.m. today I began the chemical extraction of $93^{239}$ from 210 gm of repurified UNH which has been irradiated since Tuesday with slow neutrons produced from a total exposure of 3100 microampere-hours of deuterons on beryllium at the 60-inch cyclotron (bombardment no. 93-16). I extracted the uranium with ether, precipitated from the aqueous phase rare earth fluorides to carry the $93^{239}$. I then dissolved this precipitate and carried out an oxidation-reduction cycle using $\mathrm{BrO}_{3}{ }^{-}$as oxidizing agent and $\mathrm{SO}_{2}$ as reducing agent. I completed this at $6: 05 \mathrm{p} . \mathrm{m}$.

Saturday, April 26, 1941

Starting at 3:30 p.m. Wahl carried my $93^{239}$ of yesterday through another oxidation-reduction cycle to furthex purify it. Then I came in at $8: 45 \mathrm{p} . \mathrm{m}$. to test the efficiency of our oxidation-reduction cycle procedure. I took a portion $\left(5.3\right.$ microcuries) of this $93^{239}$ sample, added it to a solution of $3 \mathrm{gm} \mathrm{UNH}$ in $40 \mathrm{CC}$ water, and carried out $1-1 / 2$ oxidation-reduction cycles using $\mathrm{BrO}_{3}{ }^{-}$as oxidizing agent and $\mathrm{SO}_{2}$ as reducing agent, stopping at 1l:00 p.m.

Sunday, April 27, 1941

At 10:30 a.m. I finished the second oxidation-reduction cycle which I had started yesterday, then placed the final rare earth fluoride sample containing the $93^{239}$ in a special little circular platinum dish with shallow sides and evaporated it to dryness. At 4:00 p.m. I measured the yield of $93^{239}$. I measured the beta radiation from this $93^{239}$ sample using the ionization chamber FP-54 electrometer setup and found 3.7 microcuries. Compared to the intensity of my starting sample $(5.3$ microcuries) corrected for decay ( 4.5 microcuries), this corresponds to an overall yield of about $80 \%$ for the two oxidation-reduction cycles. 
In reply to its letter of April 7, 1941 the University of California received a letter dated April 22 from F. H. Kohloss, Lieutenant Colonel, Corps of Engineers, Executive Planning Branch, Office of the Assistant Secretary of War, Room 2078, Munitions Building, Washington, D.C. regarding 2nd Lt. Arthur Charles Wahl, Jr. saying that our request for the classification of Wahl as a "key faculty member" has been received. The letter went on to say "it is regretted that after careful consideration we are unable to approve your request for the transfer of this officer to the War Department Reserve Pool. Your letter is being forwarded to the Adjutant General for formal reference to the agency responsible for determining the more limited period of deferment which may be granted to this officer."

Thursday, May 1, 1941

I sent letters to Dr. Briggs and Phil Abelson enclosing for each a copy of the letter dated April 22, 1941 from F. H. Kohloss regarding Wahl and expressing distress at this discouraging turn of events. I indicated that their reference to wahl as a "key faculty member" must be a misunderstanding, since he is not a faculty member (which has been made abundantly clear). I said the Army does not seem to want to understand about "key research" in universities.

Saturday, May 3, 1941

Today Wahl completed his measurements on the rate of growth of the $94^{239}$ alpha particles in sample no. 93-15-3 isolated on April 3, using our ionization chamber-linear amplifier-magnetic field setup. Although the counting rate is rather low, and hence the amount of alpha growth is small, he succeeded in demonstrating that the alpha particles do grow in at a rate consistent with the known half-life of 2.3 days for the parent beta-emitting $93^{239}$.

This again demonstrates directly the genetic relationship between beta emitting $93^{239}$ and its alpha emitting daughter $94^{239}$, which had been inferred as the result of the less complete measurements by Kennedy on the growth of $94^{239}$ alpha activity in sample $\mathrm{A}$.

Monday, May 12, 1941

This afternoon wahl undertook to remove the bulk of the rare earth carrier (approximately $6 \mathrm{mg}$ ) from our Sample $A$ in order to improve our accuracy for measuring the fission cross section and the intensity and range of its alpha particles due to $94^{239}$. We have finally acquired a large centrifuge, required for this operation, as a result of my success in persuading Dean G. N. Lewis that limited College of Chemistry research funds should be used for this purpose. 
Wahl has investigated various methods, including electrodeposition, of preparing thin uniform samples of elements 93 and/or 94 . The best and most reliable method he found is centrifugation of a few tenths of a milligran of $\mathrm{LaF}_{3}$ carrier containing the 94 in its reduced oxidation state onto a flat-bottomed platinum dish (about $1 \mathrm{~cm}$ in diameter) clamped at the bottom of a specially designed bakelite centrifuge tube. Yesterday Wahl successfully tested the procedure with tracer amounts of $93^{239}$ and our 94 alpha emitter produced from U plus deuterons.

Working in room 305, old Chemistry Building, wahl removed the little platinum dish containing Sample A from the cardboard backing with acetone and a spatula. The bottom of the dish was cleaned with acetone to remove all of the Duco cement. The little dish was then placed in a larger platinum evaporating dish, and the sample of $\mathrm{LaF}_{3}$ and $\mathrm{CeF}_{3}$ was dissolved in concentrated $\mathrm{H}_{2} \mathrm{SO}_{4}$ and $\mathrm{HNO}_{3}$ with heating and several evaporations to fumes of $\mathrm{SO}_{3}$. After diluting the sulfuric acid with water and removing the little dish, while washing it with water, about $300 \mathrm{mg}$ of $\mathrm{K}_{2} \mathrm{~S}_{2} \mathrm{O}_{8}$ and $10 \mathrm{mg}$ of $\mathrm{Ag}^{+}\left(\mathrm{AgNO}_{3}\right)$ were added, and the solution heated gently for 15 minutes, when the brown color of $\mathrm{Ag}^{+2}$ was fully developed (along with the yellow color of $\left(\mathrm{e}^{+4}\right.$ ). Then $\mathrm{HF}$ was added, the suspension digested briefly, cooled, and centrifuged. The supernatant solution was evaporated in a platinum dish to about $10 \mathrm{cc}$, cooled, and a cold solution of $\mathrm{SO}_{2}$ was added; then the solution was evaporated to fumes of $\mathrm{SO}_{3}$. After cooling and diluting, $0.2 \mathrm{mg}$ of $\mathrm{Ce}^{+++}$was added, then $\mathrm{HF}$, and the suspension was centrifuged in the specially designed bakelite centrifuge tube with a new little flat-bottom platinum dish at the bottom for collection of the small amount of $\mathrm{CeF}_{3}$ containing $94^{239}$.

We call this new sample "Sample B." We estimate its thickness as $0.16 \mathrm{mg} / \mathrm{cm}^{2}$, which should be sufficiently thin for our purposes.

Sunday, May 18, 1941

Continuing experiments started last night, today Kennedy, Segrè and I made our definitive measurements on the thinned sample B (produced by Wahl from Sample A last Monday). The measurements were made at the 37-inch cyclotron using the neutrons produced by the bombardment of the beryllium target by $8 \mathrm{Mev}$ deuterons. Sample B was placed near the screened window of our ionization chamber imbedded in the improved paraffin arrangement near the beryllium target.

Comparison of its fission rate was made with that of a more accurately determined sample containing 200 micrograms of $\mathrm{U}^{238}$, and hence 1.46 micrograms of $U^{235}$ (U Standard No.4) prepared by electrodeposition by wahl last evening. A deuteron beam of about 9 microamperes was used and measurements with both Sample B and U Standard No. 4 were made, with and without the slow neutron shielding material cadmium plus boron. Sample B gave a fission rate with slow neutrons of 80 per minute, while our 1.46 microgram $U^{235}$ sample, when placed in an identical position, gave a fission rate with slow neutrons of 140 per minute. 
This now enables us to calculate the slow neutron fission cross section of $94^{239}$ compared to that of $\mathrm{U}^{235}$. For this we need a more accurate weight for the $94^{239}$ in Sample B. Our measurement of the intensity of beta particles from the $93^{239}$ in Sample $A$ (from which Sample $B$ was derived) was 52 millicuries when measured at $5: 35 \mathrm{p} . \mathrm{m}$. on March 6 . When this is corrected for decay, this corresponds to 120 millicuries at 10:00 p.m. on March 3, the time when the $93^{239}$ was separated from the $94^{239}$ which had grown in up to that time. 120 millicuries of $93^{239}$ with its 2.3 day half-life corresponds to 0.5 micrograms, which is also the weight of the daughter $94^{239}$.

The slow neutron fission cross section of $94^{239}$ is therefore equal to $\frac{1.46}{0.5} \times \frac{80}{140}=1.7$ times that of $U^{235}$.

With the thin sample it is also possible to measure the alpha counting rate of $94^{239}$. This is now 800 counts per minute compared to 240 counts per minute before the thinning. Correcting for the geometrical factor (1.3\%) for our ionization chamber-magnetic field setup, as determined with a known amount of uranium of the same thickness as the sample, the total alpha emission of the sample now amounts to about 60,000 per minute. This corresponds to an alpha half-life for $94^{239}$ of about three times $10^{4}$ years. Our absorption measurements on the alpha particles indicate a range in air $\left(15^{\circ} \mathrm{C}\right.$ ) of the order of 3.6 or $3.7 \mathrm{~cm}$ corresponding to an energy of about $5.2 \mathrm{Mev}$.

NOTE: Segrè and I presented Sample B to the Smithsonian Institution at a public ceremony on March 28, 1966, the 25th Anniversary of the first date on which the slow neutron fission of $94^{239}$ was demonstrated. It had been stored in a cigar box most of the intervening 25 years. The sample, in its cigar box, has been on display at the Smithsonian Institution since the date of this presentation.

Monday, May 19, 1941

We told Lawrence about our definitive demonstration yesterday of the slow neutron fissionability of $94^{239}$ and he was quite excited. He immediately phoned the University of Chicago to give the news to Arthur C. Compton, who is serving as Chairman of the National Academy of Sciences Review Committee (the other members are E. O. Lawrence, J. C. Slater, John H. Van Vleck and W. D. Coolidge), which is evaluating the U.S. uranium program in order to determine if more funds and facilities would better serve the national defense. Compton made an immediate attempt to phone (unsuccessfully) and then sent a telegram to Vannevar Bush, Chairman of the National Defense Research Committee. (It was Bush and the National Defense Research Committee that made the request to the National Academy of Sciences to review the U.S. uranium effort.) In his telegram Compton indicated that the demonstration of the fissionability of element 
94 greatly increases the importance of the fission problem since the available material is thus increased by over 100 times; this could eliminate the need for uranium isotope separation if the chain reaction to produce element 94 can be achieved. He said that Alfred Loomis and Ernest Lawrence accordingly have requested him to urge anew the vital importance of pushing the work at Columbia and Chicago with all vigor and promptness.

Tuesday, May 20, 1941

Today, using some of the 94 alpha emitter from the third uranium plus deuteron bombardment (no. 93-14), wahl attempted an electrochemical deposition from HCl solution by reduction with zinc. Using $0.5 \mathrm{mg} \mathrm{sn}{ }^{++}$ carrier in the solution, the addition of amalgamated zinc led to the codeposition of less than $0.1 \%$ of element 94 , indicating that element 94 is quite electro positive.

Thursday, May 22, 1941

I wrote a letter to McMillan bringing him up to date on the present status of our information on the new element 932.1 -day beta emitter and its 94 alpha emitting daughter.

Monday, May 26, 1941

Lawrence received a letter dated May 23, 1941 from Compton today indicating that he had sent a telegram to Dr. Bush (a copy of which he enclosed) immediately after Lawrence's phone call last Monday. He indicated that he has already gotten an indication from Briggs that as a result of this increased action is underway. He also questioned the conclusion that $\mathrm{U}^{238}$ is really all subject to fission as a result of the demonstrated fissionability of $94^{239}$, pointing out that this conclusion assumes adequate neutron emission in the $94^{239}$ fission process.

I wrote to John Hamaker giving him a list of suggestions concerning micro-chemistry equipment that we might purchase or have built in our shops preparatory to his coming to work with us in the fall.

Tuesday, May 27, 1941

Lawrence wrote to Compton saying that he would not have changed one word of the telegram dispatched to Bush on Monday, May 19, even though the question of neutron emission from $94^{239}$ has not been answered at the present time. He expressed confidence that the number of neutrons emitted per fission of $94^{239}$ will prove to be even greater than for $U^{235}$. 
Today Lawrence, Segrè, Kennedy and I wrote a letter to Dr. Briggs reporting on our results on the fission of $94^{239}$ with slow neutrons. We said that the cross section for fission of $94^{239}$ with slow neutrons is about 1.7 times that of $\mathrm{U}^{235}$ and described in some detail our experiments that have led to this conclusion. We also reported that $94^{239}$ decays with a half-life of about $3 \times 10^{4}$ years by the emission of alpha particles which have a range in air $\left(15^{\circ} \mathrm{C}\right.$.) of the order of 3.6 or $3.7 \mathrm{~cm}$.

NOTE: The essential information in the letter to Briggs was given to Professor Gregory Breit in his capacity with Physical Review in Chicago on May 1, 1942 to be placed in the files of Physical Review for post-war publication. This paper was published as an article in the Physical Review entitled "Properties of 94(239)" by J. W. Kennedy, G. T. Seaborg, E. Segrè and A. C. Wahl, Phys. Rev. 70, 555 (1946). (October 1 and 15 issue).

Thursday, June 5, 1941

Segrè, Wahl and I started a bombardment of $1.9 \mathrm{~kg}$ of repurified UNH with neutrons produced by $16 \mathrm{Mev}$ deuterons on beryllium in the 60-inch cyclotron. The purpose of this bombardment is to produce a new larger sample of $94^{239}$ in order to measure its fission cross section for fast neutrons (as well as slow neutrons). The UNH is wrapped in twelve cellophane tubes and placed in a large paraffin block immediately behind the beryllium target.

Monday, June 9, 1941

Today Robert Underhill, Secretary and Treasurer of The Regents of the University of California, received from Irvin Stewart, Secretary, National Defense Research Committee, a letter dated June 5, 1941 enclosing a proposed contract with $G$. N. Lewis and me as Official Investigators covering the search for elements 93 and 94 in nature. This contract is to be signed on behalf of The Regents and returned. The final magnitude of the support for this investigation is $\$ 2,000$ and it covers the period May 1, 1941 to December 1, 1941.

Thursday, June 12, 1941

Lawrence received today a letter from Dr. Lyman J. Briggs dated June 10, 1941 requesting information on the yields, specific activities and radiation of $\mathrm{U}^{237}$ that can be produced by bombardment of uranium with neutrons at the Berkeley 60-inch cyclotron. They need this information because they are considering using $\mathrm{U}^{237}$ as a tracer in uranium isotope separation work. In order to do this, the range of the $U^{237}$ beta 
particles is important because too short a range will make the method inaccurate due to any irregularities in the film of $\mathrm{U}_{3} \mathrm{O}_{8}$ in which the intensity of $\mathrm{U}^{237}$ must be determined.

Monday, June 16, 1941

I received a letter dated June 14, 1941 from Vannevar Bush informing me that I have been appointed official Investigator to be responsible jointly with $\mathrm{Dr}$. G. N. Lewis in connection with a contract between the University of California and the National Defense Research Committee (he was referring to our contract covering the search for elements 93 and 94 in nature). He also enclosed for my signature and return copies of a Pledge of Secrecy and Oath of Allegiance. He asked that the Oath of Allegiance be taken before a Justice of the Peace or Notary Public.

Tuesday, June 17, 1941

I sent back to Washington my Pledge of Secrecy and notarized Oath of Allegiance as requested in the letter from Bush.

I wrote to Dr. Lyman J. Briggs in response to his letter to Lawrence of June 10, 1941 to give him some information on $U^{237}$ which we already have on hand on the basis of previous work. I enclosed copies of absorption curves taken with our air-filled aluminum ionization chamber connected to a FP-54 vacuum tube electrometer, and a decay curve taken with a Lauritsen quartz-fiber electroscope. I said that the beta particles are rather soft with a half thickness at the beginning of the absorption curve of approximately $6 \mathrm{mg} / \mathrm{cm}^{2}$ of $\mathrm{Al}$. I indicated that as a result of our bombardment of $1.2 \mathrm{~kg}$ of UNH cour bombardment of February 28-March 3, 1941) we determined a yield for $\mathrm{U}^{237}$ of 0.0045 microcuries per gram of uranium element per microampere-hour. I pointed out that with smaller amounts of uranium, higher specific activities are obtained and indicated that we will try to develop a szilard-Chalmers method of concentration to achieve even higher specific activities.

Wednesday, June 18, 1941

We started the bombardment of $5 \mathrm{gm}$ of repurified UNH (bombardment no. 93-17) with neutrons produced by $16 \mathrm{Mev}$ deuterons on beryllium in the 60-inch cyclotron for the purpose of obtaining information on $\mathrm{U}^{237}$ as requested by Dr. Briggs in his letter to Lawrence of June 10, 1941.

Friday, June 20, 1941

We completed this afternoon the bombardment of UNH (bombardment no. 93-17) to produce $U^{237}$ with a total neutron exposure corresponding to 
2600 microampere-hours of deuterons. Wahl performed ether extractions and recrystallizations of $\mathrm{UNH}$ in order to isolate a radiochemically pure sample. He made measurements on a weighed portion of this purified UNH sample during the evening and determined the yield of $\mathrm{U}^{237}$ to be 0.05 microcuries per microampere-hour per gram of uranium element, using our calibrated electroscope no. 3 (sensitivity 3.1 divisions per second per microcurie-assumed the same as for $93^{239}$ ) and correcting for self-absorption, absorption in the cellophane covering the sample and in the window of the electroscope.

Wahl has finally heard from the Adjutant General's office of the war Department, in a letter dated June 12, 1941, that he has been transferred to the War Department Reserve Pool; we finally can breathe more easily that he will not be called to active duty with the consequent serious impact on our research program.

Saturday, June 21,1941

Wahl initiated an experiment using some of the $U^{237}$-containing UNH from bombardment no. 93-17 to determine whether the $U^{237}$ exchanges with uranium in salicyladehyde-orthophenylenediamine-uranyl. The purpose is to see whether salicylaldehyde-orthophenylenediamine-uranyl is suitable for use in the szilard-Chalmers method for concentration of $\mathrm{u}^{237}$.

We completed the neutron bombardment of $1.9 \mathrm{~kg}$ of UNH (bombardment no. 93-18), which was started on June 5, with a total of 14,000 microampere-hours of deuterons. Compared to the neutron bombardment, utilizing $1.2 \mathrm{~kg}$ UNH and 3368 microampere-hours, which produced Sample B (containing 0.5 microgram of $94^{239}$ ), we expect about

$$
0.5 \cdot \frac{1.9}{1.2} \cdot \frac{14,000}{3368}=3 \text { micrograms of } 94^{239}
$$

(actually somewhat more because 0.5 microgram doesn't represent all of the $94^{239}$ produced in the bombardment that led to sample $B$; the $94^{239}$ in Sample B all came from the intermediate parent $93^{239}$ which was isolated after the end of the rather long bombardment).

Sunday, June 22,1941

Wahl made measurements on his $\mathrm{U}^{237}$ exchange experiment initiated yesterday and found essentially complete exchange in about 20 hours at room temperature in his water-chloroform system. He then repeated the experiment and found nearly complete exchange in times as short as five minutes, indicating that salicylaldehyde-orthophenylenediamine-uranyl is not satisfactory under these conditions for application to the szilard-Chalmers method for concentration of $\mathrm{U}^{237}$. However, we are not sure of the purity of our organic uranyl compound. 
During the last couple of months Gofman has been experimenting with methods to prepare by electrolysis thin samples of $\mathrm{Pa}^{233}$ suitable for measuring the growth of daughter $\mathrm{U}^{233}$ alpha activity. He has prepared two samples of strength 6 microcuries and 10 microcuries as measured with our calibrated FP-54 ionization chamber. It is necessary for Gofman to return to his home in Cleveland, ohio because of our inability to find funds to support him for the summer. He has been supported during the school year on his small stipend as a teaching assistant which is insufficient to take care of his subsistence needs for the remainder of the summer. He is asking Wahl and English to make alpha particle measurements on his electrolyzed 6 microcurie and 10 microcurie samples to look for the growth of daughter $\mathrm{U}^{233}$ during his absence.

Thursday, June 26, 1941

Robert Underhill received a letter from Irwin Stewart dated June 24, 1941 enclosing a signed copy of the contract dated June 14, 1941 between the National Defense Research Committee and The Regents of the University of California covering the search for elements 93 and 94 in nature. stewart indicated that we will be informed later about the number to be assigned to this contract.

Sunday, June 29, 1941

Gofman left today to return to his home in Cleveland, ohio to spend the remainder of the summer before the fall semester opens in August.

Monday, June 30,1941

Robert Underhill received from Irvin stewart today a letter dated June 28, 1941 informing him that our contract covering the search for elements 93 and 94 in nature has been assigned contract number NDCrc-197, Symbol No. 180. The effective date of this contract was May 1, 1941.

I wrote to $\mathrm{Dr}$. Lyman J. Briggs to give a further report on the yield of $\mathrm{U}^{237}$ based on the results of our bombardment (no. 93-17) of $5 \mathrm{gm}$ of UNH on June 18-20, 1941. I indicated the yield is 0.05 microcuries per microampere-hour per gram of uranium element, a specific activity about 10 times as great as that obtainable with a large amount $(1.2 \mathrm{~kg})$ of UNH. I also said that work is underway to develop the Szilard-Chalmers process in order to increase the specific activity. 
Due to the availability of funds from the Lewis-Seaborg contract NDCrc-197, I am able today to start Wahl on summer employment so that he can continue his work during the summer. He will work at a rate of 60 cents an hour, and we have funds to pay him for 85 hours in July and 85 hours in August. He will, of course, work much more than this, but we are limited by availability of funds to pay him for all his work time. Up till now he has been supporting himself on the basis of income from his teaching assistantship.

Unfortunately, I do not have a similar source of funds to support the work of Jack Gofman on the $\mathrm{U}^{233}$ problem and therefore he found it necessary to return to his home in Cleveland, Ohio to spend the summer.

Wednesday, July 2, 1941

Yesterday and today Segrè and I made attempts to measure the fast neutron fission cross section of $94^{239}$ using sample B. (Kennedy left early last month to assume a temporary, or perhaps permanent, position with American Cyanamid Company in Stamford, Connecticut; we are, howevery, protecting his position as instructor here at Berkeley in the event he should elect to rejoin us here in the fall, which we hope will be the case.) During June Segrè and I constructed a completely new ionization chamber and linear amplifier for use in our contemplated fast neutron fission measurements. This chamber has quartz rather than hydrogen-containing insulators in order to.eliminate insofar as possible hydrogenous material which might give rise to slow neutrons which would mask the fast neutron effect. The ionization chamber is constructed so that the sample whose fission cross section is being measured can be placed on the inside so as to have maximum efficiency for the detection of the fission fragments. The ionization chamber is completely surrounded by cadmium in order to protect our samples from slow neutrons. We are using as our source of neutrons for these fission measurements, as usual, the 37-inch cyclotron which furnishes $8 \mathrm{Mev}$ deuterons for bombardment of a beryllium target. The ionization chamber containing the sample under investigation is placed as close as possible to the beryllium target.

Unfortunately, we find that Sample B contains too small an amount of $94^{239}$ (0.5 microgram) to make possible measurement of its fast neutron fission despite repeated attempts.

Wednesday, July 9,1941

Working on the porch outside room 305, Old Chemistry Building, today Wahl began his chemical ioslation of $94^{239}$ from the $1.9 \mathrm{~kg}$ UNH bombarded with neutrons from June 5-21 (bombardment no. 93-18). Now that we understand the chemistry of element 94 so well, he intends to isolate the $94^{239}$ directly rather than through the intermediate $93^{239}$ as was done in the production of sample $B$, thus potentially increasing the yield since 
none will be lost due to decay of $93^{239}$ during the bombardment. He experienced difficulty in removing the cellophane bags from the paraffin block because some broke in the process, but he succeeded in making an ether extraction to reduce the volume of UNH and then a second ether extraction of the aqueous portion to further reduce the amount of UNH. The aqueous portion from this second ether extraction contains the $94^{239}$ and fission products in a greatly reduced amount of UNH, and this is his starting solution for the isolation of $94^{239}$.

Thursday, July 10, 1941

Today I wrote to Dr. Lyman J. Briggs at Professor Lawrence's suggestion to propose some additional work that might be done on the measurement of fission cross sections in the uranium and transuranium regions under a research contract for which I might act as official Investigator. I suggested that the proposed work might be divided into three groups: (1) the measurement of the slow neutron fission cross section of $93^{239}$; (2) the measurement of the slow neutron fission cross section and the fast neutron fission cross section of $93^{237}$ (on the assumption that we can isolate this as the daughter of 7-day betadecaying $\mathrm{U}^{237}$ ); (3) the measurement of the slow neutron fission cross section and the fast neutron fission cross section of $\mathrm{U}^{23}$ (the expected daughter, formed by the beta decay of $\mathrm{Pa}^{233}$ and $\mathrm{Th}^{233}$ which results from the capture of slow neutrons by ordinary stable $\mathrm{Th}^{232}$ ). I indicated that the first two of these projects could probably be handled without any additional paid help, but the $U^{233}$ project could best be done with the additional help of a paid chemist Ph.D. assistant. All the projects would need some funds for equipment, materials and supplies, and to pay for the time of use of the 60-inch cyclotron. I suggested a total budget of $\$ 21,000$.

Sunday, July 13,1941

Wahl completed his isolation of $94^{239}$ from the UNH and fission products which served as his starting solution on Wednesday, having carried out during the intervening days a very rigorous chemical separation procedure. The procedure involved oxidation-reduction steps using rare earth fluoride carrier and included separations from $93^{239}$ in steps utilizing $\mathrm{BrO}_{3}{ }^{-}$which oxidizes element 93 and not element 94 . The $94^{39}$-containing rare earth fluoride was reprecipitated a number of times in order to effect complete separation from uranium. The chemical procedure included eight separations from uranium, three separations from theorium and fission products, and two separations from element 93. The final sample of $94^{239}$ (on platinum sheet in a copper "dish" with cellophane covering) is present with cerium fluoride in which the thickness amounts to $0.16 \mathrm{mg} / \mathrm{cm}^{2}$, which should be very suitable for fission and alpha particle counting. Wahl first designated this as Sample D, but I suggested we change the designation to Sample $F$ to avoid the similar sounds of B (for our Sample B) and D (the use of $C$ and $E$ was eliminated 
on the same basis). Measurements (with the magnetic field off) of its alpha activity with our low geometry ionization chamber-linear amplifiermagnetic field outfit, which we now designate as $\mathrm{W}-1$, indicate 4300 alpha counts per minute which converts through the use of the measured geometrical effeciency (approximately $2 \%$ ) to a total alpha particle emission. rate of 3500 per second. This corresponds to a weight of $94^{239}$ of about $3-1 / 2$ micrograms for a half-life of $3 \times 10^{4}$ years. Due to the high counting rate it was necessary to make this measurement through calibrated screens to cut down the transmission of alpha particles to the ionization chamber; measurement of a small aliquot of the solution before the cerium fluoride precipitation served as a check of the total emission rate. This sample should be very suitable for the measurement of the cross section for the fission of $94^{239}$ with fast neutrons, which is a very important property in connection with its potential use as a nuclear explosive.

NOTE: There was a mistake of a factor of 2 in our calculation of the weight of $94^{239}$ in sample $F$ from its alpha disintegration rate; the weight as calculated from these data should be about 1-3/4 micrograms.

Wednesday, July 16,1941

Measurements by Wahl and English on Gofman's electrolyzed 6 and 10 microcurie $\mathrm{Pa}^{233}$ samples have not been able to establish the growth of any daughter $U^{233}$ alpha particles, undoubtedly due to the small intensity of the samples and the long half-life of $U^{233}$. This is consistent with the measurements of April 23 which suggest that the half-life of $U^{233}$ is $10^{5}$ years or longer.

Thursday, July 17,1941

Since July 13, when our larger $94^{239}$ sample (Sample F) became available, Segrè and I have been making renewed attempts to measure the fast neutron fission cross section of $94^{239}$. We have made many improvements in our quartz insulated ionization chamber and our linear amplifier and are operating with the ionization chamber surrounded by cadmium and boron carbide to prevent slow neutrons reaching our sample. As a result of tests during recent weeks, we have found that it is not possible to use very large beams of deuterons because of the intense ionization from neutron recoil atoms in the chamber (a condition which did not exist to nearly so great an extent in our slow neutron experiments). This condition is aggravated by the emission by our $94^{239}$ sample of 3,500 alpha particles per second so that it is necessary to conduct test blank runs with a polonium sample of equal alpha activity in order to define operating conditions.

In our experiments we are comparing the fission rate in our sample $F$ ( 3.5 micrograms of $94^{239}$ ) with our uranium Standard No. 5, prepared by 
Wahl through the electrolysis of natural uranium out of absolute alcohol solution. This uranium standard contains 200 micrograms of uranium and hence effectively 200 micrograms of $\mathrm{U}^{238}$ to undergo fission with fast neutrons.

Our experiments this afternoon were very successful. The 3.5 microgram $94^{239}$ sample gave a fission rate of six per minute when the neutrons from a two microampere beam of deuterons on beryllium were used at the 37-inch cyclotron. The uranium standard gave a fission rate of 100 per minute with a two microampere beam of deuterons under conditions identical to those of the $94^{239}$ measurements. Hence the fission cross section of $94^{239}$ for fast neutrons is $200 / 3.5 \times 6 / 100=3.4$ times that of uranium $\left(\mathrm{U}^{238}\right)$ for our fast Be plus deuteron neutrons.

NOTE: When the correctly calculated weight (about $1-3 / 4$ micrograms) for the $94^{239}$ in sample $F$ is used in this calculation, the value of the ratio of the fast neutron fission cross section of $94^{239}$ to that of $U^{238}$ is twice as large.

Friday, July 18, 1941

From 5:00 to 11:15 p.m. Wahl made a bombardment of $615 \mathrm{gm}$ of UNH with the neutrons produced by the bombardment of beryllium with 60-inch cyclotron deuterons for 370 microampere-hours (bombardment no. 93-19). The purposes of this bombardment include the isolation of a very pure $93^{239}$ fraction in order to make an accurate determination of its half-life, and the measurement of the growth of the daughter $94^{239}$ alpha particles using our ionization chamber-linear amplifier-magnetic field setup.

Saturday, July 19, 1941

Wahl isolated from yesterday's neutron-bombarded UNH, through a series of oxidation-reduction cycles using rare earth fluoride, a very pure sample of $93^{239}$ to be used for the measurement of its decay using our ionization chamber connected to the FP-54 electrometer, and the growth of daughter $94^{239}$ alpha particles, using our ionization chamberlinear amplifier-magnetic field setup $(\mathrm{W}-1)$, for a determination of the half-life of $94^{239}$. He used $0.2 \mathrm{mg}$ Ce for the final fluoride precipitate, dissolved this in $\mathrm{H}_{2} \mathrm{SO}_{4}$ solution, diluted to $25 \mathrm{cc}$, took a $1 \mathrm{cc}$ aliquot for use in calibration experiments (sample no. 93-19-G), then reprecipitated the $\mathrm{CeF}_{3}$ containing $96 \%$ of the $93^{239}$ (sample no. 93-19-1) which he mounted on platinum sheet in a copper "dish" and covered with cellophane. He measured the intensity of the beta radiation from sample no. 93-19-1 (placed on the second step) with our ionization chamber FP-54 electrometer setup through $50.5 \mathrm{mg} / \mathrm{cm}^{2}$ of Al absorber and found a galvanometer deflection of $2.4 \times 10^{8} \mathrm{~mm}$, allowing for the absorption in the $\mathrm{Al}$ and cellophane and converting to the "standard" resistance 
and shunt setting R2Sl. He used aliquots from sample no. 93-19-G to make measurements, using the same ionization chamber, on the beta radiation of $93^{239}$ with a sample mounted on platinum and an identical sample mounted on thin $(2.4 \mathrm{mg}$ per sq. $\mathrm{cm}$ ) Al; correcting for the very small backscattering from the thin-aluminum (with the help of a measurement made with the Al-mounted sample in the inverted position), he finds that the correction factor for the backscattering of $93^{239}$ beta radiation from the platinum backing place is 0.62 .

He also started to measure the growth of alpha particles using our ionization chamber-linear amplifier-magnetic field setup $(W-1)$ which has a measured geometrical efficiency of about $2 \%$, and found an initial counting rate of 102 alphas per minute, indicating incomplete separation from daughter $94^{239}$ during the chemical isolation procedure.

Wednesday, July 23,1941

I received a copy of a letter dated July 21, 1941 from Robert Underhill to Mr. Lundberg, University Chief Accountant, indicating that on July 4, 1941 the National Defense Research Committee has been reconstituted as "Office for Emergency Management, Office of Scientific Research and Development," with Dr. Vannevar Bush as Director. All contracts and acts of the National Defense Research Committee will be taken over by the new organization.

Thursday, July 24, 1941

Today Segré and I wrote to Dr. Lyman J. Briggs reporting our results on the fission of $94^{239}$ with fast neutrons. We indicated that our recent work shows that the cross section of $94^{239}$ with fast neutrons is about 3.4 times that of uranium (the natural mixture of isotopes), which is probably equivalent to saying that it is about 3.4 times that of $\mathrm{u}^{238}$. We described the chemical process by which the new sample of $94^{239}$, upon which these measurements were made, was isolated and the method that we used to measure the fast neutron fission cross section with emphasis on our precautions to eliminate any disturbing effect of slow neutrons. We emphasized that this fast neutron fission cross section of $94^{239}$ is characteristic of our particular energy spectrum of fast neutrons; that is, fast neutrons, from $8 \mathrm{Mev}$ deuterons on beryllium, some of which have suffered one or more reflections from the water in the shielding tank and from the magnet cooling oil in the vicinity of the target.

NOTE: A report incorporating the main contents of our letter to Dr. Briggs was issued almost immediately by the Uranium committee in washington. This bore the report number $\mathrm{A}-22$ and had the title "Fast Neutron Fission of $94^{239 "}$ with the authors indicated as G. T. Seaborg and E. Segrè. 
Today I visited with Dr. Lyman J. Briggs, Gregory Breit and Phil Abelson in Washington, D.C., to make a personal report on our work at Berkeley for the Uranium Committee and to indicate some of our plans for the future. I discussed with Breit the problem of measuring the spontaneous fission rate of $94^{239}$ which is so important in connection with its use as a nuclear explosive.

Professor Lawrence received a letter from Dr. Vannevar Bush, Director, Office of Scientific Research and Development, dated August 1, 1941 informing us that the contract I requested in my letter of July 10 , 1941 to Dr. Briggs has been approved in the amount of $\$ 21,500$; it has been assigned the number OEMsr-206, symbol No. 319. It is effective August 1,1941 and will run one year to July 31, 1942.

Friday, August 8, 1941

Wahl has completed the measurement of the growth of daughter $94^{239}$ alpha particles in sample no. 93-19-1, started on July 19, and finds a final intensity of 170 alpha particles per minute, or a total growth corresponding to 68 alpha particles per minute.

Today we received delivery of another Lauritsen electroscope purchased from the Fred C. Henson Co.-this will be our electroscope no. 6 .

Saturday, August 9, 1941

Professor Joel H. Hildebrand, Chairman of our Department of Chemistry, received a letter dated August 6, 1941 from Dr. Briggs informing him that the National Defense Research Committee is assigning an important project of the Uranium Section to me involving the transfer of $\$ 21,500$ for its active prosecution. He indicates that it is essential that this work be expedited in every way possible and suggests that I be relieved from teaching duties as far as possible during the coming year.

Monday, August 11, 1941

The University of California Administration received today a letter from Irvin Stewart informing us that our project for the production and measurements of the properties of $94^{239}$ (which has the contract NDCrc-201) has been assigned an A-1-a priority rating by the Priorities Division of the office of Production Management. This means that we will have top priority in purchasing key items for our project in competition with other national demands for our needed items. This is the contract, put into effect recently, to cover the cost of cyclotron operations to produce our two $94^{239}$ samples, designated $A$ (changed to $B$ ) and $F$, used for the measurement of the slow and fast neutron fission cross sections of $94^{239}$, 
as well as the cost of other related cyclotron bombardments; it was arbitrarily back-dated to April 1, 1941 and extends until October 1, 1941.

Friday, August 15, 1941

Today I visited with Mr. Gilbert LaBine, Dr. W. R. Horn, and Mr. Carl French of the Eldorado Gold Mines Ltd. in Toronto and with the head chemist, Dr. Pochon, and Dr. Horn at the Port Hope Refinery of Eldorado Gold Mines Ltd. The purpose of my visit is to decide whether in their chemical process for extracting uranium from pitchblende ore any transuranium elements that might be present could be found. I discussed the flow sheet for their refining process and explored the possibility of their sending some pitchblende concentrate and some chemical fractions from their ore processing to Berkeley for use in our search for transuranium elements in nature. I, of course, did not reveal my objective to any of these people.

Tuesday, August 19, 1941

I visited Dr. Clarence $\mathrm{W}$. Balke at the Fansteel Metallurgical Corporation in Chicago and discussed with him the possibility of shipping some tantalite, samarskite, and hafnium ores to Berkeley. Again, I have in mind the search for transuranium elements in these ores, but of course did not reveal my objective to Balke.

Wednesday, August 20, 1941

John Hamaker arrived in Berkeley and is ready to start his graduate work with me.

Friday, August 22, 1941

Gofman returned from his home in Cleveland, Ohio to resume his graduate research program, courses and teaching assistantship at Berkeley.

Saturday, August 23, 1941

Wahl completed decay measurements on sample no. 93-19-1 isolated on July 19. He found a very consistent exponential decay over the entire period, indicating a half-life of 2.35 days for $93^{239}$. 
I received from Robert Underhill, Secretary and Treasurer of The Regents of the University of California, a letter enclosing a patent form transferring all my patent rights under the Lewis-Seaborg contract NDCrc-197 to The Regents of the University of California. I have decided to ignore this and not sign and return it to The Regents, and I believe that Professor Lewis has decided the same.

Classes for the fall semester are beginning today. I am teaching two freshman chemistry laboratory sections and am also serving as the Department of Chemistry advisor for graduate students; i.e., I am responsible for the administration of Chemistry 280.

Norman Bonner, an undergraduate chemistry major, is starting to take Chemistry $180 \mathrm{H}$ (undergraduate research course) under my direction this semester. I plan to have him investigate the chemical separation of thorium, rare earths and uranium with the ultimate plan of developing methods of separating $\mathrm{UX}_{1}$ (as a source of $\mathrm{U}^{234}$ ) from large quantities of UNH.

Thursday, August 28, 1941

In view of the increasing requirements of our research program, and the need to replace some of our older models, we received today three more Lauritsen electroscopes purchased from the Fred C. Henson Co.-these will be nos. $7,8 \& 9$.

Friday, August 29, 1941

Wahl today made a rough calculation of the half life of $94^{239}$ using the data from sample no. 93-19-1. The growth of daughter $94^{239}$ alpha particles from July 19 to August 8 corresponds to 68 per minute. Using the approximate geometrical efficiency $(2 \%)$ of our ionization chamberlinear amplifier-magnetic field outfit (W-1), this corresponds to an absolute alpha particle intensity of 56 per second. Using the measured initial intensity of $93^{239}$ beta radiation of July 19 (galvanometer deflection $2.4 \times 10^{8} \mathrm{~mm}$ corresponding to resistance and shunt setting R2S1), our present best calibration of our ionization chamber FP-54 electrometer $\left(1.3 \times 10^{4} \mathrm{~mm}\right.$ per microcurie of $93^{239}$ beta radiation, our determination of January 11, 1941, corrected for the backscattering of the thin glass backing material), and correcting for the backscattering of the $93^{239}$ beta radiation from platinum (a factor of 0.62 ), we find for the absolute intensity of $93^{239}$ :

$$
\frac{2.4 \times 10^{8}(0.62)}{1.3 \times 10^{4}}=11.4 \text { millicuries. }
$$

Combining this with the measured intensity of the alpha particles of daughter $94^{239}$, we find for the half-life of $94^{239}$; 


$$
\frac{\left(11.4\left(3.7 .10^{7}\right)(2.35)\right.}{(56)(365)}=5 \times 10^{4} \text { years. }
$$

Tuesday, September 2, 1941

Dr. Raymond w. Stoughton, a Berkeley Ph.D. of last year, started to work with me today in a postdoctoral capacity supported by the funds from my new contract no. OEMsr-206 which includes a provision of $\$ 2,500$ to pay for a postdoctoral chemist for one year; he will work in room 311, Gilman Hall, which has been made available to my group.

Wednesday, September 3,1941

Stoughton began work today to try to improve the methods for the extraction of $\mathrm{Pa}^{233}$ from neutron bombarded thorium nitrate. He and Gofman plan to establish the best conditions for the carrying of $\mathrm{Pa}^{233}$ by zirconium phosphate and other carriers and will also attempt to perfect the conditions for electroplating the final samples of $\mathrm{Pa}^{233}$ which we plan to isolate in order to allow its growth to daughter $U^{233}$. The aim is to develop an overall procedure that can be used to isolate as large as possible samples of $\mathrm{U}^{233}$ from large quantities of thorium nitrate subjected to long neutron bombardments at the 60-inch cyclotron.

\section{Friday, September 5, 1941}

Today a target of uranium telluride was bombarded with 16 Mev deuterons in the 60-inch cyclotron for 550 microampere-hours by Joe Hamilton. The dual purpose is to provide iodine-131 for Hamilton and a source of our 94 alpha emitter $\left(94^{238}, 94^{236}\right.$ or $\left.94^{235}\right)$ for wahl. According to our new system of nomenclature, this bombardment has been designated U,D-1; this more general system of nomenclature recognizes the expansion of our program to include our other investigators who will have cyclotron bombardments. After the target was dissolved in nitric acid with the removal of iodine by distillation by Hamilton, the solution was given to wahl for use in the isolation of the element 93 and 94 fractions.

Tuesday, September 9, 1941

Wahl has performed two oxidation-reduction cycles using $\mathrm{BrO}_{3}^{-}$as oxidizing agent, $\mathrm{SO}_{2}$ as reducing agent and rare earth fluoride as carrier to iolate a 93 fraction from uranium-deuteron bombardment U,D-1 performed on september 5, 1941. He mounted three-fourths of the 93 fraction on a platinum plate in a copper "dish" and under cellophane (sample no. U,D-l-I) and one-quarter on cellophane backing (sample no. 
U,D-1-4). These element 93 samples contain a mixture of beta emitting $93^{239}$ and the 2.0 -day 93 isotope $\left(93^{238}, 93^{236}\right.$ or $\left.93^{23.5}\right)$.

Stoughton today tested the carrying of $\mathrm{Pa}^{23} 3$ by $\mathrm{MnO}_{2}$, formed by the oxidation of $\mathrm{Mn}^{++}$by $\mathrm{ClO}_{3}^{-}$, and found good results. This method for coprecipitating protoactinium was developed by A. V. Grosse and B. Agruss (J. Am. Chem. Soc. 57, 438 [1935]).

Wednesday, September 10, 1941

This afternoon, using 93 sample no. U,D-1-1, Wahl performed absorption measurements in lead on the gamma radiations using our ionization chamber FP-54 electrometer. He finds a half thickness for the high energy tail of $10.0 \mathrm{gm} / \mathrm{cm}^{2}$, corresponding according to Gentner's data (Physik zeit 38 , 836 [1937]) to a gamma-ray energy of $1.07 \mathrm{Mev}$, due to the 2.0 -day 93 isotope $\left(93^{238}, 93^{236}\right.$ or $\left.93^{235}\right)$.

He also started to measure the decay of sample U,D-1-1 without absorbers using our ionization chamber FP-54 electrometer, and through lead absorber ( $16.7 \mathrm{gm}$ of lead $/ \mathrm{cm}^{2}$ ) using our Lauritsen electroscope no. 5 . The initial intensity on the ionization chamber FP-54 (with the sample on step 2) corresponds to a galvanometer deflection of $4.13 \times 10^{7} \mathrm{~mm}$ (corrected back to the time of separation from daughter 94) after conversion to our "standard conditions" resistance and shunt setting R2S1; using our calibration of $1.3 \times 10^{4} \mathrm{~mm}$ deflection per microcurie, this corresponds roughly to a total intensity of 3 millicuries, but a better calibration of our FP-54 ionization chamber is required in order to establish a more accurate value.

Wahl also started measurements on the growth of alpha particles in 93 sample no. U,D-1-1 using our ionization chamber-linear amplifiermagnetic field arrangement $(W-1)$ to bend out the intense 93 beta radiation; he is making concurrent measurements on the daughter 94 alpha particles from "standard" sample no. 93-14-11, isolated on March 15 from our third deuteron bombardment of uranium, in order to monitor the sensitivity of the counting equipment.

Thursday, September 11, 1941

WahI made absorption measurements in aluminum using our ionization chamber FP-54 electrometer on 93 sample no. U,D-1-4 (the mixture of $93^{239}$ and the 2.0-day 93 isotope $\left[93^{238}, 93^{236}\right.$ or $\left.93^{235}\right]$ ) mounted on cellophane. He finds an end point of $385 \mathrm{mg}$ of aluminum $/ \mathrm{cm}^{2}$ corresponding to a beta energy of $1.02 \mathrm{Mev}$ according to Feather's rule, $\mathrm{R}\left(\mathrm{gm} \mathrm{Al} / \mathrm{cm}^{2}\right)=0.543$ $E$ (Mev) - 0.160; this, then, represents the upper energy limit of the beta particles from the 2.0-day 93 isotope because the beta particles of $93^{239}$ present in the sample are completely absorbed by smaller thicknesses of aluminum. These absorption measurements show that the ionization due to 
the electrons of the 2.0-day 93 isotope is about $5 \%$ of the total from the 2.3-day $93^{239}$ plus the 2-day 93; this estimate is so rough that an attempt to correct for backscattering is not justified. Since the total beta activity of sample U,D-1-1, corrected back to the time of separation on Tuesday, is 3.0 millicuries, as calculated using the measured efficiency of our ionization chamber for detection of the beta radiation of $93^{239}$, the intensity of the 2-day 93 component is about 150 microcuries.

Monday, September 15, 1941

Wahl bombarded 1125 grams of repurified UNH from $6: 00$ to $10: 30$ p.m. with neutrons produced by the bombardment of beryllium with $16 \mathrm{Mev}$ deuterons on the 60-inch cyclotron for a total of 1,000 microampere-hours. The bombardment was designated $\mathrm{U}, \mathrm{n}-6$ under our new system of nomenclature which recognizes the expansion of our program to include other investigators who are conducting bombardments of uranium with neutrons; not all of these bombardments, which include practice and calibration runs, will be mentioned in this record. The UNH was placed in 10 soft glass test tubes imbedded in paraffin and wahl determined the relative yields in each of the 10 test tubes by measuring the intensity of the induced gamma activity.

Tuesday, September 16, 1941

Beginning early this morning and carrying through until about 1:00 p.m., Wahl carried out two oxidation-reduction cycles using the standard $\mathrm{BrO}_{3}{ }^{-}$oxidation, $\mathrm{SO}_{2}$ reduction, and rare earth fluoride carrying, to isolate a pure $93^{239}$ fraction (free of $94^{239}$ daughter) from yesterday's neutron bombardment of UNH (bombardment no. U, n-6). He divided this 93 fraction into two portions $-95 \%$ for sample no. U, n-6-1 to be used for a measurement of the growth of $94^{239}$ alpha particles, and also a careful determination of the half-life of $93^{239}$ and absorption measurements on its gamma radiation using our ionization chamber FP-54 electrometer setup, and half of the remainder for sample no. U,n-6-2 to be used for absorption measurements to determine the energy of the beta radiation of $93^{239}$ using our ionization chamber FP-54 electrometer setup. The stronger sample $(U, n-6-1)$ is mounted on platinum in a copper "dish" and under cellophane, while the weaker sample $(U, n-6-2)$ is mounted on cellophane to eliminate beta backscattering in the absorption measurements on beta particles.

He measured the intensity of the beta radiation of the more intense $93^{2.39}$ sample $(\mathrm{U}, \mathrm{n}-6-1)$, placed on step 2 , through $50.5 \mathrm{mg} / \mathrm{cm}^{2}$ of Al absorber, with our FP-54 ionization chamber, and found a galvanometer deflection of $1.26 \times 10^{9} \mathrm{~mm}$ (correcting to the time of separation from daughter $94^{239}$ ), allowing for the absorption in the $\mathrm{Al}$ and cellophane and converting to the "standard conditions" of resistance and shunt setting R2S1. Our FP-54 ionization chamber should be better calibrated for the beta radiation of $93^{239}$ in order to translate this into an absolute intensity. 
In the afternoon, using the stronger $93^{239}$ sample $(U, n-6-1)$, wahl made absorption measurements of the gamma rays in lead using our ionization chamber FP-54 setup. The gamma ray absorption corresponds to a complex of gamma ray energies over the energy range $0.1-0.3 \mathrm{Mev}$.

Wednesday, September 17,1941

Today Wahl started to measure the growth of $94^{239}$ alpha particles in $93^{239}$ sample no. U,n-6-1 using our ionization chamber-linear amplifiermagnetic field setup $(W-1)$; he is making concurrent measurements on sample no. 93-19-1, a $93^{239}$ sample isolated on July 19 and now fully grown to $94^{239}$, to monitor the sensitivity of the detection equipment.

Completing his measurements on the decay on the mixture of 93 isotopes formed in the deuteron bombardment (sample no. U,D-1-I) of September 5, Wahl finds a half-life of 2.05 days. Although this is a mixture of $93^{239}$ and the other isotope of element $93\left(93^{238}, 93^{236}\right.$ or $\left.93^{235}\right)$, the half-life corresponds to the decay of the latter because the measurements were made through $16.7 \mathrm{gm}$ of lead $/ \mathrm{cm}^{2}$, a thickness that effectively eliminates all the radiations from $93^{239}$.

Gofman has resumed his electrolysis experiments in order to develop methods for preparing thin samples of $\mathrm{Pa}^{233}$. This is preparatory to the production of a large sample of $\mathrm{Pa}^{233}$ through the extensive neutron bombardment of a large quantity of thorium nitrate, which we plan to carry out as soon as the chemical extraction methods have been perfected.

I received from Gregory Breit a letter dated September 15, 1941 referring to my conversation with him in Washington on August 4, 1941 in which we discussed the need to measure the spontaneous fission rate of $94^{239}$. The interest in this property of $94^{23.9}$ arises in connection with its potential use as an explosive because the higher the rate of spontaneous fission, the faster one will have to bring the parts of the bomb together in order to prevent a premature explosion. He says that according to British results, ordinary uranium undergoes spontaneous fission at a rate of 5 per second per kilogram, which corresponds to 0.3 per minute per gram; if the spontaneous fission is due to $\mathrm{U}^{235}$, the $\mathrm{U}^{235}$ undergoes spontaneous fission at a rate of about 40 per minute per gram. He says that it is extremely important to determine the spontaneous fission rate of $94^{239}$ and asks us to undertake this task.

Thursday, September 18,1941

This afternoon and evening wahl made aluminum absorption measurements on $93^{239}$ sample no. U, n-6-2 (mounted on cellophane) using our ionization chamber FP-54 electrometer setup. He finds that the beta end point comes at about $260 \mathrm{mg}$ of aluminum $/ \mathrm{cm}^{2}$, corresponding to an upper, energy limit of the beta particles of 0.78 Mev according to Feather's rule, $\mathrm{R}\left(\mathrm{gm} \mathrm{Al} / \mathrm{cm}^{2}\right.$ 
$=0.543 \mathrm{E}(\mathrm{Mev})-0.160$. The initial part of the absorption curve shows a half thickness of $9.5 \mathrm{mg} / \mathrm{cm}^{2}$. As in the earlier absorption measurements, he observes a high rationearly 1000 to 1 -of beta-radiation to gamma-ray ionization.

I received from Gregory Breit a copy of the report on the slow neutron fission properties of $94^{239}$ that Lawrence, Segrè, Kennedy and I sent to Dr. Lyman J. Briggs in the form of a letter on May 29, 1941, which has been issued by the Uranium Committee as report $A-33$ under the authoriship of Lawrence and me.

Tuesday, September 23,1941

I wrote to Gilbert LaBine and Carl French of Eldorado Gold Mines Ltd., Toronto, Canada, to ask them to send me a simple flow sheet covering their process for refining uranium ores and to send me 10 pounds of pitchblende concentrate from the Great Bear Lake region of Canada where they have their uranium mine. The possibility of these requests was discussed during my visit with them on August 15, 1941.

Dr. M. L. Oliphant visited today with a number of us, including Professor Lawrence, to discuss progress on the uranium project in England and its effect on the U.S. program. In the course of our conversations, he told me that Dr. Shenstone has a quantity of ionium and that I might be able to obtain some of this material from him.

Wednesday, September 24,1941

We are starting a bi-weekly seminar with meetings at 4:00 p.m. on alternate Wednesdays to review advances in nuclear chemistry and physics that are taking place throughout the world. In addition to all the people working with Kennedy and me (that is, Friedlander, Wahl, Gofman, English, Hamaker, Stoughton, Bonner and Leitz), this will be attended by other graduate students of the Berkeley Department of Chemistry working in these fields: Paul Nahinsky, T. H. Norris (working with Dr. Samuel Ruben), David Altman (with Professor George E. Gibson), C. Barter (with Professor Willard Libby), R. B. Duffield (with Professor Melvin Calvin), as well as Edwin F. Orlemann, instructor in chemistry. At the first meeting today J. W. Kennedy made a survey report on use of radioactive tracers in exchange reactions; C. Barter made a literature report on "Chemical Reactions Induced by Neutrons" (Hagiwara and Suito, Proc. Imperial Academy (Tokyo) 16, 543 [1940]); and G. Friedlander made a literature report on "Mass Spectrographic Determination of Mass Number of Artificially Radioactive Atoms" (Yamaguchi, Proc. Phys. Math. Soc. Japan 23, 264 [1941]). 
I received another letter from Gregory Breit reiterating the importance of determining the spontaneous fission rate of $94^{239}$. He believes that even if we can only establish an upper limit on the probability of spontaneous fission using our small (approximately microgram) samples of $94^{239}$, this is very much worthwhile doing.

Friday, September 26, 1941

I wrote to Gregory Breit in Washington saying that we will be glad to go ahead and make observations on the spontaneous fission of $94^{239}$. I indicated that since we have only 3.5 micrograms of $94^{239}$, it will take some time to establish whether its rate of spontaneous fission is similar to that of $\mathrm{U}^{235}$, assuming that spontaneous fission in natural uranium is due to $\mathrm{U}^{235}$. I indicated that if the spontaneous fission rate of $94^{239}$ is equal to that of $\mathrm{U}^{235}$, we should expect about one spontaneous fission event per five days of counting.

We ordered 25 pounds of thorium nitrate from the Fairmont Chemical Co. for use in our planned neutron bombardments to produce a large sample of $\mathrm{U}^{233}$.

Wednesday, October I, 1941

Dr. Morris Perlman started to work with me in room 311, Gilman Hall. I am asking him to work on the problem of searching for elements 93 and 94 in uranium ores. He will be paid under my contract NDCrc-197, which expires December 1, 1941, and then continue under contract OEMsr-206 at a salary of $\$ 1,800$ per year. He obtained his Ph.D. at Berkeley last year and has been working on an isotope separation process with Dr. C. G. Maier in the Hearst Mining Building during much of the intervening time. I shall ask him to start to practice our chemical methods for the separation of elements 93 and 94 preparatory to the receipt of the sample of pitchblende concentrate which we have ordered from the Port Hope Refinery of Eldorado Gold Mines Ltd.

Saturday, October 4, 1941

Stoughton has performed numerous experiments during the last month to establish the best conditions for the carrying of $\mathrm{Pa}^{233}$ by zirconium phosphate out of thorium nitrate solutions.

Wednesday, October 8,1941

Wahl completed the measurement of the growth of $94^{239}$ alpha particles in $93^{239}$ sample no. $\mathrm{U}, \mathrm{n}-6-1$ started on September 17 , using our low 
geometry ionization chamber-linear amplifier-magnetic field setup ( $W-1$ ). He finds that the alpha particles grow in at a rate corresponding to a half-life of 2.3 days for the parent $93^{239}$ (when alpha counter sensitivity corrections are made through use of the data obtained with 93-19-1 monitor sample). The alphas have grown to a total intensity of 260 counts per minute; since the geometrical efficiency of the arrangement is of the order of 2\%, the absolute alpha intensity is some 50 times larger than this and will have to be more precisely determined by making measurements using an ionization chamber in which the sample can be placed inside on one of the electrodes; this has the advantage that its geometrical efficiency can be determined by counting the alpha particles of a weighed sample of uranium placed in the identical position.

He also completed measurements on the growth of alpha particles in 93 sample U,D-1-1 begun on September 10, the sample containing a mixture of $93^{239}$ and the other 93 isotope $\left(93^{238}, 93^{236}\right.$ or $\left.93^{235}\right)$. He finds that these alpha particles (due predominantly to the daughter isotope $94^{238}$, $94^{236}$ or $94^{235}$ ) have grown into the sample at a rate corresponding to a half-life of 2.0 days for the parent $93^{238}, 93^{236}$ or $93^{235}$ (when corrections are made using the data from the 93-14-11 monitor sample). This is reasonable because due to its shorter half-life, the alpha particles of $94^{238}, 94^{236}$ or $94^{235}$ should predominate over those of $94^{239}$ even though the beta emitting $93^{239}$ originally predominates in the U,D-1-1 sample. The total intensity of alpha particles has grown to a disintegration rate of 712 per minute (as measured with our setup of about $2 \%$ efficiency). Determination of an accurate absolute alpha disintegration rate here also awaits measurements with a calibrated "inside" alpha ionization chamber.

Thursday, October 9, 1941

Today Stoughton verified that a precipitate of zirconium phosphate incorporating tracer $\mathrm{Pa}^{233}$ can be dissolved in $\mathrm{HF}$ with good recovery of the $\mathrm{Pa}^{233}$ in the solution and negligible loss on a concurrent precipitate of thorium fluoride.

Monday, October 13, 1941

I wrote to R. M. Wilke of Palo Alto, California to order $1-1 / 2$ pounds of pitchblende ore from Joachimsthal, Czechoslovakia; I have learned of his possession of this ore through previous correspondence.

Today Wahl made a rough calculation of the alpha half-life of the deuteron-produced 94 using the data obtained with sample no. U,D-1-1 upon which measurements were made between september 10 and October 8 . The total growth of alpha particles corresponds to an intensity of 590 alpha particles per second based on the $2 \%$ geometrical efficiency for our ionization chamber setup. The corresponding initial intensity of 
the parent 93 beta particles was 150 microcuries (an estimated 5\% of the roughly determined 3 millicuries) as measured on september 10 on the ionization chamber FP-54 setup. This, then, corresponds to a half-life of

$$
\frac{(150)\left(3.7 \times 10^{4}\right)}{590} \frac{(2.0)}{365}=50 \text { years. }
$$

Due mainly to the uncertainty of estimating the proportion of beta particle radiation in the mixture of $93^{239}$ and the 2.0 -day 93 parent which is due to the 2.0-day parent lestimated to be $5 \%$ on the basis of the aluminum absorption measurements of September Il, the potential error in the alpha half-life is quite large and so we suggest that the half-life be considered to be $50 \pm 25$ years. This, then, is our best value for the half-life of the alpha emitting daughter of the beta emitting $2.0-$ day $93\left(93^{238}, 93^{236}\right.$ or $93^{235}$ ) and we shall for convenience refer to this 94 isotope, so useful in our tracer chemical investigations of 94 , as the "50-year 94 isotope."

Wahl also made another rough calculation of the half-life of $94^{239}$, using the data obtained with sample U,n-6-1. From the initial beta particle intensity of the parent $93^{239}$ measured on september 16 (galvanometer deflection of $1.26 \times 10^{9} \mathrm{~mm}$ corresponding to resistance and shunt setting R2S1), our calibration of the ionization chamber FP-54 electrometer setup ( $1.3 \times 10^{4} \mathrm{~mm}$ per microcurie) and correcting for the backscattering of the $93^{239}$ beta radiation from platinum (a factor of 0.62 ), he finds for the initial intensity of the $93^{239}$ beta radiation:

$$
\frac{\left(1.26 \times 10^{9}\right)(0.62)}{1.3 \times 10^{7}}=60 \text { millicuries. }
$$

Using the intensity of daughter $94^{239}$ alpha particles measured on October 8 (260 counts per minute) and our best estimate of the geometrical efficiency of our ionization chamber-linear amplifier-magnetic field setup (approximately 2\%), he finds for the half-life of $94^{239}$ :

$$
\frac{(60)\left(3.7 \times 10^{7}\right)(60)(0.02)(2.33)}{(260)} \cong 6 \times 10^{4} \text { years. }
$$

Tuesday, October 14, 1941

We received 10 pounds of pitchblende ore from Eldorado Gold Mines Ltd. in response to my request of september 23 and also a flow sheet of the uranium refining process.

Wednesday, October 15, 1941

Wahl took 53 grams of the pitchblende ore recelved from Eldorado Gold Mines Ltd. yesterday and dissolved it by repeated treatment with concentrated $\mathrm{HNO}_{3}$, aqua regia, $\mathrm{H}_{2} \mathrm{SO}_{4}, \mathrm{HCl}$, and $\mathrm{HF}$. The purpose is to 
dissolve as much as possible, and he succeeded in dissolving nearly all, and then to analyze the resulting solution to establish the possible presence of isotopes of element 93 and 94 .

Saturday, October 18, 1941

Wahl completed the isolation of the element 93 and 94 fractions from the 53 grams of pitchblende ore. His measurements show, within their accuracy, the presence of no alpha particles or beta particles that can be attributed to the presence of isotopes of elements 93 or 94 .

Monday, October 20, 1941

Stoughton is commencing experiments to develop an improved procedure for the removal of $\mathrm{Pa}^{233}$ from large quantities of neutron-bombarded thorium nitrate through the initial use as carrier of manganese dioxide, produced by the reaction of $\mathrm{Mn}^{++}$with permanganate ions.

Wednesday, October 22, 1941

Having skipped our bi-weekly seminar two weeks ago, today we met and heard a general survey on slow neutrons by Emilio segrè and a report by Kennedy on particles with $36 \mathrm{Mev}$ energy produced in the 60-inch cyclotron.

Thursday, October 23, 1941

Herbert N. McCoy, now living in Los Angeles, visited us and presented us with a millicure of mesothorium which we have requested in correspondence with him.

Monday, October 27, 1941

Using apparatus set up in room 120, Gilman Hall, during the last few weeks Wahl and Kennedy have been making observations on the spontaneous fission of $94^{239}$ in response to the letters of September 15 and september 26, 1941 from Gregory Breit. On the basis of the results to date using our Sample F (3.5 micrograms), they have established that the rate of spontaneous fission of $94^{239}$ is less than $1 / 10$ spontaneous fission per hour per microgram. I sent a letter to Gregory Breit of the Uranium Committee in Washington, with a carbon copy to Dr. Lyman J. Briggs, reporting this result and saying that the observations will be continued with new, more stable apparatus in order to increase the sensitivity. 
Wahl completed today his decay measurements on $93^{239}$ (sample no. $U, n-6-1)$ begun on September 16 and obtained a value of $2.33 \pm 0.03$ days.

Today Kennedy and Wahl moved their apparatus for observations on the spontaneous fission of $94^{239}$ to East Hall, an old abandoned building situated on the south side of Faculty Glade. (This is the building in which, 4-1/2 years ago, my graduate student partner, David C. Grahame, and I did many of our Geiger-Mueller counter experiments on our Ph.D. thesis problems involving the inelastic scattering of fast neutrons because we, also, required an isolated environment.) The reason for this more isolated site is to be more free of electrical interference in their counting circuits, which must exhibit extraordinary stability in order to establish, reliably, the absence of spontaneous fission counts over long periods of observation.

Thursday, October 30, 1941

Today Gofman and Stoughton began the bombardment of $5 \mathrm{~kg}$ of dissolved thorium nitrate with neutrons from the deuteron bombardment of beryllium at the 60-inch cyclotron. The thorium nitrate is surrounded by 3-5 inches of paraffin (except between the sample and the beryllium target). It has been completely purified from any content of natural $\mathrm{Pa}^{231}$ by three separate manganese dioxide precipitations. It is planned to measure the growth of $\mathrm{U}^{233}$ alpha particles from the chemically isolated $\mathrm{Pa}^{233}$ parent, which is the reason for the removal of the natural 30,000-year alpha emitting $\mathrm{Pa}^{231}$ from the thorium nitrate. The isolation procedure also contemplates the removal of natural uranium as well as thorium from the $\mathrm{Pa}^{233}$ so that it may decay to pure daughter $\mathrm{U}^{233}$.

Friday, October 31,1941

I wrote to Clarence W. Balke, Fansteel Metallurgical Corporation, North Chicago, Illinois, inquiring whether his tantalite ore contains uranium and rare earths and indicating that we would like to receive from him tantalite ores as well as samarskite and hafnium ores.

Monday, November 3, 1941

Morris Perlman has completed his preparations for undertaking the search for elements 93 and 94 in our pitchblende ore from the Great Bear Lake region of Canada and is today starting to dissolve about $400 \mathrm{gm}$ of the pitchblende concentrate. 
Gofman and Stoughton have developed a method for extracting Pa 233 from a large volume of thorium nitrate solution through coprecipitation on manganese dioxide formed by the reaction of manganous chloride with potassium permanganate in hot solutions. This can then be followed by dissolving the manganese dioxide and further concentrating the $\mathrm{Pa}^{23}$ by coprecipitation with zirconium phosphate and dissolving the zirconium phosphate in HF. They developed their procedure for reducing the volume of carrier by repetitions of this cycle and then finally isolating the $\mathrm{Pa}^{233}$ by electrolysis. Thus, they are ready to perform the chemical extraction of the $\mathrm{Pa}^{233}$ from the $5 \mathrm{~kg}$ of thorium nitrate whose bombardment with neutrons at the 60-inch cyclotron started on October 30 .

Saturday, November 8,1941

I wrote to Dr. Lyman J. Briggs pointing out that our contract for studies and experimental investigations in connection with a search for elements 93 and 94 in nature (contract NDCrc-197) expires on December 1 and our allotment of $\$ 2,000$ has been largely consumed. I indicated that we have a number of ores coming so that I would like to continue the program and to augment it by hiring a Ph.D. chemist to participate with us. (Actually, I have already hired Morris Perlman to work on this.) I submitted a proposed budget of $\$ 7,000$, including $\$ 3,000$ for the salary of the Ph.D. chemist, $\$ 1,500$ for University overhead expenses, and $\$ 2,500$ for equipment and travel. I indicated that our results so far are negative, but that we have only treated amounts of the order of 50 grams of ores and that we will now use larger amounts.

Monday, November 10, 1941

The neutron bombardment of $5 \mathrm{~kg}$ of thorium nitrate (in saturated aqueous solution) which commenced on October 30 was terminated today after a total of 14,250 microampere-hours of deuterons on beryllium at the 60-inch cyclotron. Gofman and stoughton immediately started their extraction procedure.

During the last few weeks Joseph G. Hamilton has isolated about 300 millicuries of Po 210 from residue concentrates obtained in the processing of uranium which have been shipped to us from the Port Hope Refinery of the Eldorado Gold Mines Ltd. He has mixed the Po 210 with beryllium and placed this in a suitable container in order to provide us with a Po-Be neutron source. This has the great advantage that it is relatively free of gamma rays in contrast with radon-beryllium or radium-beryllium neutron sources. Hence, it can be used more effectively for testing the efficiency of our counting circuits for amplification of fission pulses from the ionization chamber in which our samples are placed to measure spontaneous fission rates. This efficiency is tested by placing a sample of fissionable isotope in the ionization chamber and then inducing fissions at an easily measurable rate through the use of a neutron source; the Po-Be neutron source is especially suitable for this purpose. 
We now have in operation through the efforts of Kennedy and English a rather versatile array of ionization chambers, accompanying amplifiers and scale of eight recording circuits with cenco counters. Continuing in operation is our screened window alpha counting ionization chamber (designated no. W-1) equipped with a magnet for bending out high intensity beta particles that might be present in the alpha particle emitting samples; this can also be used as a "low geometry" alpha detector, with or without intervening screens, with the magnetic field off. For the measurement of alpha emitting samples of intermediate intensity, we have an ionization chamber (designated no. W-2) with an arrangement to have the samples placed near the screened window; this setup also has provision for interposing additional screens between the sample and ionization chamber to allow measurement of samples of higher intensity. In addition, for determining the absolute counting rate of alpha-particle-emitting samples we have an ionization chamber of the"inside" type (designated no. W-3) in which the sample is placed on one electrode inside the ionization chamber; the geometrical efficiency of this has been determined to be $45 \%$ by counting the alpha particles of a weighed uranium sample (200 micrograms) placed in the identical position.

Wednesday, November 12,1941

Today Kennedy and Wahl began, with their apparatus in East Hall, what is planned to be a long time observation of the spontaneous fission rate of $94^{239}$ using Sample F (3.5 micrograms) and new and improved ionization chambers and linear amplifiers. The $94^{239}$ is mounted on a platinum-coated copper dish and placed on one electrode of the ionization chamber. Calibrations with the use of our Po-Be neutron souxce and a thin uranium sample indicate that about $70 \%$ of the fissions are being recorded under the operating conditions of the experiment. In order to increase our confidence in the experiments, they will use two separate ionization chamber and linear amplifier setups in order for each of them to conduct his separate series of spontaneous fission experiments.

Using a radon-beryllium source (containing about 150 millicuries of radon obtained from Professor Don Yost at Caltech) in East Hall, Wahl began further measurements to compare the slow neutron (cadmium absorbable) fission cross section for $94^{239}$ with that of $\mathrm{U}^{235}$. The radon-beryllium neutron source and the ionization chamber in which this sample is placed on one of the electrodes are completely surrounded with paraffin in order to produce slow neutrons, and measurements are to be made with and without the sample surrounded with cadmium to establish the effect of slow (cadmium absorbable) neutrons. Wahl is using as his $94^{239}$, Samples $F$ and $B$, and for his $\mathrm{U}^{235}$, a new Uranium Standard No. 6 containing 200 micrograms of natural uranium (and hence 1.4 micrograms of $\mathrm{u}^{235}$ ). In the course of this work he will remeasure the weights, through alpha counting, of $94^{239}$ in Samples $F$ and $B$ using our $W-1$ ionization chamber-1inear amplifier-magnetic field outfit (through our calibrated screen no. 2, with magnetic field off); he will make comparative measurements using the known absolute alpha emission rate of sample 93-19-1 (9,170 alpha disintegrations per minute) which he has determined by careful measurements using our calibrated "inside" ionization chamber (designated no. W-3). 
The present best conversion factors for converting the readings of the galvanometer (in $\mathrm{mm}$ ) to the standard $R$ (resistance) and $S$ (shunt) setting of R2SI when observed at other $R$ and $S$ settings are as follows:

\begin{tabular}{|c|c|c|c|c|}
\hline & R2 & R3 & R4 & R5 \\
\hline Sl & 1.00 & 10.03 & $1.16 \times 10^{2}$ & $1.26 \times 10$ \\
\hline S2 & 3.27 & 32.8 & $3.78 \times 10^{2}$ & $4.12 \times 10^{3}$ \\
\hline s3 & 9.90 & 99.5 & $1.14 \times 10^{3}$ & $1.25 \times 10^{4}$ \\
\hline S4 & 30.6 & $3.07 \times 10^{2}$ & $3.54 \times 10^{3}$ & $3.86 \times 10^{4}$ \\
\hline S5 & 95.5 & $9.60 \times 10^{2}$ & $1.10 \times 10^{4}$ & $1.20 \times 10^{5}$ \\
\hline
\end{tabular}

Friday, November 14,1941

Wahl completed his measurements of the slow neutron fission cross section of $94^{239}$ using Samples $F$ and $B$ and the Uranium Standard No. 6 source of $\mathrm{U}^{235}$. He obtained values for the cross section for the slow neutron fission of $94^{239}$ relative to that of $U^{235}$ (cross section of $94^{239}$ a little more than half that of $\mathrm{U}^{235}$ ) which are substantially smaller than that obtained earlier (last May) by Kennedy, Segrè and me. From his alpha measurements today with ionization chamber $\mathrm{W}-1$ and screen no. 2 , he finds (in counts per minute) the values: Sample F, 456; Sample B, 169; Sample 93-19-1, 23. Using the known absolute disintegration rate of sample 93-19-1 (9,170 alpha disintegrations per minute) and using his recent (October 13) value for the half-life of $94^{239}\left(6 \times 10^{4}\right.$ years), this yields the values 1.2 micrograms for the weight of $94^{239}$ in sample $B$ and 3.3 micrograms in Sample $F$.

Wahl finds the following results in terms of fission counts per hour for slow (cadmium absorbable) neutrons: Sample B, 12; Sample F, 30; Uranium Standard No. 6, 23. This corresponds to a ratio of the slow neutron fission cross section of $94^{239}$ to that of $U^{235}$, for sample $B$ $1.4 \times 12 / 1.2 \times 23=0.6$, and for Sample $F 1.4 \times 30 / 3.3 \times 23=0.55$.

Since we used the value 0.5 micrograms (based on a half life of $3 \times 10^{4}$ years) for the weight of $94^{239}$ in Sample $B$ in our calculations last May, the difference in the weights accounts for the difference in our ratios of slow neutron fission cross sections. The resolution of this discrepancy awaits a better determination of the half life of $94^{239}$ and we intend to do this.

This demonstrates how much more convenient it is to use such a neutron source rather than the neutrons furnished by the 37-inch cyclotron as in our earliex fission experiments with Samples B and $F$ (in March-May and July). More convenient yet would be a Ra-Be source of neutrons and we intend to acquire such a source to contiue these and to perform our additional contemplated fission measurements. 
Gofman and Stoughton completed today their extraction of $\mathrm{Pa}^{233}$ from the $5 \mathrm{~kg}$ of neutron-bombarded thorium nitrate. This procedure, begun last Monday, started by dissolving thorium nitrate in 26 liters of water which was adjusted to a nitric acid concentration of $0.5 \mathrm{M}$. To this solution about $400 \mathrm{gm}$ of manganous chloride was added. This was precipitated from the hot solution in three separate portions by the addition of potassium permanganate to the solution. Each precipitate of manganese dioxide was centrifuged out separately. The protactinium is carried down with the manganese dioxide, more or less quantitatively, under these conditions.

The combined manganese dioxide precipitates were dissolved in a mixture of hydrogen peroxide and hydrochloric acid. After decomposing the hydrogen peroxide by boiling, about $200 \mathrm{mg}$ of zirconium oxychloride was added to this solution, the zirconium was precipitated as zirconium phosphate by the addition of phosphoric acid, and the precipitate was centrifuged out. Several further precipitations of zirconium phosphate were performed by adding furthur 100-mg portions of zirconium oxychloride, each precipitate being centrifuged out separately. Zirconium phosphate carries doen the protactinium essentially quantitatively.

It was then necessary to remove the $\mathrm{Pa}^{23}$ from the rather large amount of zirconium with which it was present and to purify it from uranium and thorium. The zirconium and protactinium phosphates were brought into solution by treatment with dilute hydrofluoric acid. This step also gave a further separation from thorium which was precipitated as the insoluble fluoride at this point. This solution was cooled with ice water, and zirconium and protactinium hydroxides were precipitated by adding dilute sodium hydroxide solution; the solution must be kept cold in this precipitation or difficulty. will be experienced in redissolving the precipitated hydroxides. The hydroxide precipitate was then dissolved in nitric acid, and the protactinium was carried away from most of the zirconium by another series of manganese dioxide precipitations. Some of the zirconium came along with the manganese dioxide precipitate in this procedure so that a further removal of the zirconium, as well as the manganese, from the protactinium was necessary. This involved going through the above described cycle two more times, finally ending up with a small zirconium phosphate precipitate containing the protactinium.

This zirconium phosphate precipitate, which now contained only about $10 \mathrm{mg}$ of zirconium, was dissolved in hydrofluoric acid and, after precipitation of the hydroxide as described above, was converted to the nitrate by dissolving the hydroxide in nitric acid. Another precipitation of the zirconium phosphate was then made in order to be sure that all the uranium and thorium in amounts less than a microgram were removed. The final zirconium phosphate precipitate was made at 11:59 p.m.

Kennedy has put into operation a thick-windowed ionization chamber filled with freon $\left(\mathrm{CF}_{2} \mathrm{Cl}_{2}\right)$, window thickness of $120 \mathrm{mg} \mathrm{Al} / \mathrm{cm}^{2}$, connected to our FP-54 electrometer, especially suitable for detecting gamma rays. Using a mesothorium source, we have compared the efficiency of this chamber with that of our air-filled ionization chamber and find that the freon chamber has a sensitivity for gamma rays which is 5 to 7 times 
greater than that of the air chamber; the higher ratio (7) corresponds to the higher energy gamma rays as determined by the use of aluminum absorbers.

I received a letter dated November 14, 1941 from Dr. Iyman J. Briggs acknowledging my letter of November 8, 1941 and indicating that the question of continuing our work with ores will be taken up at the next meeting of the Uranium Section of the Office of Scientific Research and Development.

Tuesday, November 18,1941

Continuing their work, Gofman and Stoughton converted last night's final zirconium phosphate precipitate (containing all of the $\mathrm{Pa}^{233}$ recovered from their large bombardment) to zirconium sulfate and dissolved this in $0.33 \mathrm{M}$. ammonium fluoride solution preparatory to isolation of the $\mathrm{Pa}^{233}$ by electrolysis onto copper plates. The first electrolysis began at 11:00 a.m. and continued until 8:15 p.m. This sample, which is labeled $T h+n-I-P a-I$, should contain the majority of the $\mathrm{Pa}^{233}$. They then started their second electrolysis at 8:30 p.m. and continued until 9:45 p.m., labeling this sample Th+n-I-Pa-II; this should give a small sample convenient for some of the measurements.

They started their third electrolysis at 10:00 p.m. with the intention of running it overnight.

Measurements of the alpha activity of sample Th+n-I-Pa-II, using the ionization chamber-linear amplifier-magnetic field setup $(w-1)$, commenced at 11:00 p.m. and will continue over the next several months to observe the growth of daughter $\mathrm{U}^{23}$. It is planned to measure concurrently the alpha counting rate of a $\mathrm{Pa}^{231}$ standard sample in order to monitor the sensitivity of the apparatus. The measurement showed a counting rate of 22 alphas per minute, even though the sample has just been purified, indicating the presence of a $\mathrm{Pa}$ alpha emitter; this is undoubtedly due to the 30,000-year $\mathrm{Pa}^{231}$ produced during the bombardment via the reactions:

$$
\operatorname{Th}^{232}(\mathrm{n}, 2 \mathrm{n}) \operatorname{Th}^{231} \stackrel{(25 \text { hour })}{\longrightarrow} \mathrm{Pa}^{231} \text {. }
$$

Wednesday, November 19, 1941

Gofman and Stoughton completed a third electrolysis of $\mathrm{Pa}^{233}$ as the end product from their large neutron bombardment of thorium nitrate at 11:00 a.m. and labeled this sample $\mathrm{Th}+\mathrm{n}-\mathrm{I}-\mathrm{Pa}-\mathrm{III}$; this should yield a $\mathrm{Pa}^{233}$ sample of intermediate intensity. They then started a fourth electrolysis at 12 noon which ran until 9:00 p.m.; they labeled this sample $T h+n-I-P a-I V$. Very rough order of magnitude measurements of the intensities of the beta emission from these $\mathrm{Pa}^{233}$ samples using our ionization chamber FP-54 electrometer setup with absorbers (to help deal with the high intensities of radioactivity) give the following results: for sample $T h+n-I-P a-I, 300$ millicuries; for Th+n-I-Pa-II, 6 millicuries;

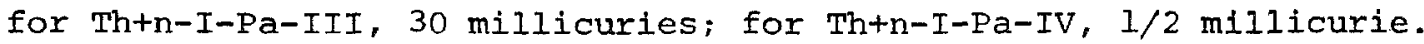


The sample Th+n-I-Pa-I will be allowed to decay until the major portion has been converted into daughter $\mathrm{U}^{23}$, after which the $\mathrm{U}^{23}{ }^{3}$ will be separated from the undecayed $\mathrm{Pa}^{233}$. The plan is to use samples $T H+n-I-P a-I I$ and -III to measure the decay and make absorption measurements on the $\mathrm{Pa}^{233}$ radiations using the ionization chamber FP-54 setup, and to determine the half-life of daughter $\mathrm{v}^{233}$ by measuring the growth of its alpha particles using the ionization chamber-linear amplifiermagnetic field setup $(W-1)$. The beta radiation from the $\mathrm{Pa}^{23^{3}}$ will be measured with Lauritsen electroscopes and with our ionization chamber connected to a FP-54 electrometer tube, depending upon the intensity of the samples. Our Lauritsen electroscopes have aluminum windows of thickness about $3 \mathrm{mg} / \mathrm{cm}^{2}$ and the FP-54 ionization chamber has an aluminum window of thickness about $0.1 \mathrm{mg} / \mathrm{cm}^{2}$. In order to determine the half-life of daughter $\mathrm{U}^{23}$, these instruments must be calibrated in an absolute manner for the radiations of $\mathrm{Pa}^{233}$ and $\mathrm{Th}^{233}$ which will be done with the help of our Geiger-Mueller counters whose geometrical efficiency is being established. Since the $\mathrm{Pa}^{23}$ radiation contains a large number of conversion electrons, it will be necessary to determine the percentage of these; this will be done through Geiger-Mueller counter measurements of the beta particles of the 25-minute $\mathrm{Th}^{233}$ and its daughter $\mathrm{Pa}^{233}$. In addition, since the $\mathrm{Pa}^{233}$ samples are mounted on copper, it will also be necessary to correct for backscattering of the beta particles.

The measurement of the beta intensity of samples $\mathrm{Th}+\mathrm{n}-\mathrm{I}-\mathrm{Pa}-\mathrm{III}$ and -II on the ionization chamber FP-54 electrometer setup will be carried out on step 2 under "standard conditions" by observing the deflection, using or converting the reading to standard resistance and shunt settings R555. The plan also is to make observations on the deflections produced by our beta standards RaE-St. - I and $\mathrm{UX}_{2}-\mathrm{St} .-4$ at the time of each measurement in order to check the sensitivity.

Measurements of the alpha activity of sample Th+n-I-Pa-III using the ionization chamber-linear amplifier-magnetic field setup ( $W-1)$ commenced at 12:30 p.m. (also to be monitored with concurrent measurements on our standard $\mathrm{Pa}^{231}$ sample). The measurement showed a counting rate of 116 alpha counts per minute, even though the sample has just been purified, indicating the presence of a $\mathrm{Pa}$ alpha emitter; as with sample $\mathrm{Th}+\mathrm{n}-\mathrm{I}-\mathrm{Pa}-\mathrm{II}$, this is undoubtedly due to the 30,000 -year $\mathrm{Pa}^{231}$ produced during the bombardment via the reactions

$$
\operatorname{Th}^{232}(n, 2 n) \operatorname{Th}^{231} \text { (25 hour) } \rightarrow \mathrm{Pa}^{231} \text {. }
$$

Tuesday, November 25,1941

Gofman conducted absorption measurements in aluminum, using the ionization chamber FP-54 outfit, on the $\mathrm{Pa}^{233}$ radiations in sample $T h+n-I-P a-I I$. He found an end point for the beta radiation of $165 \mathrm{mg}$ $\mathrm{Al} / \mathrm{cm}^{2}$, corresponding to an energy of $0.5 \mathrm{Mev}$ using the Varder \& Eddy relationship. There is evidence of soft radiation (the initial portion of the absorption curve shows a half thickness of $11.5 \mathrm{mg}$ per $\mathrm{sq} . \mathrm{cm}$ ) 
suggesting the presence of an appreciable number of conversion electrons; the observed value of 700 for the ratio of beta-particle to gamma-ray ionization is consistent with this conclusion.

Wednesday, November 26,1941

Today we received 90 pounds of $\mathrm{UNH}$ bearing the Baker and Adamson label from General Chemical Company in response to our purchase order. This will be used for the isolation of pure $U^{234}$ by extracting the $\mathrm{UX}_{1}$ and allowing it to decay to daughter $\mathrm{U}^{234}$.

Thursday, November 27, 1941

In response to my correspondence with Clarence W. Balke, Fansteel Metallurgical Corporation, of October 31, 1941, we have received samples of a rare earth-uranium columbate-tantalate ore (fergusonite) and a uranium columbate-tantalate ore (hatchettolite) for use in our program for the search for elements 94 and 93 in nature.

Friday, November 28, 1941

Kennedy and wahl completed their measurements on the spontaneous fission of $94^{239}$, using Sample $F$ (3.5 micrograms), which they started on November 12.

Wahl has found that his series of measurements with one amplifier and chamber gave zero spontaneous fission counts in 139 hours of operation. With the other ionization chamber and amplifier setup, Kennedy, during 209 hours of observation, also observed zero counts. These results enable us to make an estimate of the spontaneous fission disintegration constant of $94^{239}$, or, more convenienntly, of the "half-life" for spontaneous fission corresponding to the disintegration constant.

Using the result of zero counts in 348 hours for the two experiments taken together, the weight of the sample (3.5 micrograms), and the efficiency of the apparatus for counting fissions (70\%), we can calculate that for a half-life of $10^{13}$ years the mean time between counts would be about 20 hours, and for a half-life of $10^{14}$ years the mean time between counts would be about 200 hours. The probability of obtaining zero counts in 348 hours is about $10^{-8}$ for 20 hours mean time between counts, and about 0.2 for 200 hours mean time between counts. Therefore, we feel it is fairly safe to say that the half-life of $94^{239}$ for spontaneous fission is of the order of $10^{14}$ years or greater. This is a very important result, indicating that $94^{239}$ is probably a suitable material for use as a nuclear explosive. 
Wahl is starting to work up the various fractions from bombardment no. 93-18 (the $1.9 \mathrm{~kg}$ UNH bombarded with neutrons from June 5-21 and from which Sample $F$ was isolated on July 13) in order to recover any additional $94^{239}$, that might be available, and any 93 alpha activity (that might be due to $93^{237}$ ). He will use our standard oxidation-reduction procedures with rare earth fluoride carriers.

Monday, December 1, 1941

I sent to Gregory Breit in Washington, with a carbon copy to Dr. Lyman J. Briggs, a report by Kennedy and Wahl entitled "Search for Spontaneous Fission in $94^{239} "$ describing their experimental work and results on the 3.5 microgram sample of $94^{239}$ which have led us to the conclusion that the half-life of $94^{239}$ for spontaneous fission is of the order of $10^{14}$ years or greater. I asked whether he thinks that this work should be continued.

Today Professor Lawrence also sent a letter to Professor Compton at the University of Chicago informing him of these results of Kennedy and Wahl and urging that in view of these results, the work at Columbia University on obtaining a chain reaction with the natural mixture of uranium isotopes proceed as rapidly as possible because 94 produced by this method might be the best route to a nuclear weapon.

\section{Tuesday, December 2, 1941}

Morris Perlman has completed his dissolving of the pitchblende concentrate, a procedure that presented a number of unexpected difficulties. In order to dissolve the material it was necessary to treat it successively with a number of reagents and after each treatment the solution which was obtained was set aside and the residue was subjected to the action of the next reagent. The reagents, in the order in which they were used, were (1) boiling concentrated HCl followed by boiling aqua regia, (2) hot 6 $\mathrm{N}-\mathrm{NaOH}$, (3) hot aqua regia, (4) boiling $6 \mathrm{~N}-\mathrm{HCl}$, (5) boiling $27 \mathrm{~N}-\mathrm{HF}$ followed by $\mathrm{HNO}_{3}$ extraction, (6) fuming hot $\mathrm{H}_{2} \mathrm{SO}_{4}$ followed by $\mathrm{H}_{2} \mathrm{O}$ extraction, (7) $\mathrm{NaOH}$ fusion followed by dilute $\mathrm{NaOH}$ extraction, (8) boiling $6 \mathrm{~N}-\mathrm{HCl}$, and (9) $\mathrm{HF}$ solution followed by $\mathrm{H}_{2} \mathrm{SO}_{4}, \mathrm{Na}_{2} \mathrm{CO}_{3}$ and $\mathrm{HNO}_{3}$ solutions. After this series of treatments the final undissolved matter was entirely negligible, perhaps of the order of a few milligrams in weight. The plan is to precipitate rare earth fluoride from each of these solutions and then proceed with the isolation of fractions containing elements 94 and 93 , using our oxidation-reduction procedures with rare earth fluoride as carrier. 
Having skipped our bi-weekly seminar two weeks ago, today we had a meeting at which $C$. Barter gave a general review on radioautography; Hamaker gave a report on applications of radioactive indicators to analytical chemistry; and Norris made a literature report on "Angular Correlation of Successive Quanta Emitted from Na ${ }^{24}$ and $\mathrm{Cl}^{38}$ " (Proc. Phys. Math. Soc. Japan 23, 568 [1941]).

Thursday, December 4, 1941

Wahl has completed his isolation of 94 and 93 fractions from the residues of bombardment no. 93-18 started on Sunday. He has isolated an additional sample of $94^{239}$ (but no $93^{237}$ ) for which measurements using our ionization chamber-linear amplifier-magnetic field setup (with magnetic field off) indicate an alpha intensity about equal to that of Sample F. We are giving this the designation Sample $H$, even though a logical progression of letter designation (following Sample F) would be Sample G; however, we wish to avoid the confusion that would result from the similar sound of Sample B.

Using our air-filled ionization chamber FP-54 electrometer setup, Gofman is making beta decay measurements on samples $\mathrm{Th}+\mathrm{n}-\mathrm{I}-\mathrm{Pa}$-III and $T h+n-I-P a-I I$. Corrected back to the time of last separation from daughter $\mathrm{U}^{23} 3$ the initial $\mathrm{Pa}^{233}$ beta intensities (for the resistance and shunt setting R5S5 and with the samples on step 2) correspond to a galvanometer reading of $1795 \mathrm{~mm}$ for sample $\mathrm{Th}+\mathrm{n}-\mathrm{I}-\mathrm{Pa}-\mathrm{III}$ and $340 \mathrm{~mm}$ for sample $T h+n-I-P a-I I$. The measurements on sample $T h+n-I-P a-I I I$ are being made through $30 \mathrm{mg}$ per sq. $\mathrm{cm}$ of $\mathrm{Al}$ absorber in order to be manageable for so intense a source and the $1795 \mathrm{~mm}$ reading is a corrected one that allows for the attenuating effect of the absorber.

I received a letter from $\mathrm{H}$. T. Wensel, Technical Aide, Section S-1, dated December 2, 1974 informing me that in the future the Uranium Section of the Office of Scientific Research and Development will be designated section S-1. In the interest of secrecy, it is desired that the name uranium not be used in any circumstances when referring to this section.

Saturday, December 6, 1941

A meeting was held in Washington, D.C. by Briggs, Compton, Lawrence and Conant. The group was called together by Vannevar Bush to learn of President Franklin D. Roosevelt's reaction to the National Acedemy of Sciences committee's final report which reviewed the work on uranium and which was submitted to the President on November 27, 1941. This group was asked to constitute a new committee to be known as section s-1. Instructions to the committee were to do everything possible to find whether nuclear weapons could be made, to recommend to Bush action on fission research, be responsible for implementation of that action, and to report back its findings within six months. 
The Japanese bombed Pearl Harbor today. I heard the news on my radio while I was listening to the Chicago Bears-Chicago Cards football game in my room at the Faculty Club. The Bears had to win to tie Green Bay for the Division title; they won 34-24. The news of the Japanese attack reached the press box at halftime. There were only 18,000 fans at Comiskey Park in Chicago.

Wednesday, December 10, 1941

Stoughton began to work on the problem of separating uranium from protoactinium in order to develop a procedure for isolating the daughter $\mathrm{U}^{23}$ from the undecayed $\mathrm{Pa}^{233}$ in sample $\mathrm{Th}+\mathrm{n}-\mathrm{I}-\mathrm{Pa}-\mathrm{I}$.

Friday, December 12, 1941

From 11:15 a.m. to 11:41 a.m. today Wahl bombarded 15 grams of UNH with the neutrons obtained from the irradiation of uranium with 100 microamperes of $16 \mathrm{Mev}$ deuterons in the 60-inch cyclotron (bombardment no. $\mathrm{U}, \mathrm{n}-1 \mathrm{I})$.

One purpose of this bombardment is to establish the ratio of conversion electrons and beta particles from $93^{239}$ to those from its parent $\mathrm{U}^{239}$ in order to make a determination of the absolute beta disintegration rate of $93^{239}$. Our absorption measurements, and measurements by Carl Helmholz using his magnetic electron spectrograph, indicate that $93^{23}$ has a relatively large number of conversion electrons; it is now clear that this has led us to an overestimation of the amount of $93^{239}$ and hence an error in determining the alpha half-life of the daughter $94^{239}$ (an error in the direction of overestimating the half-life of $94^{239}$ ). Since there are presumably much fewer conversion electrons accompanying the beta decay of $\mathrm{U}^{239}$ (probably a negligible number), the genetic chain $\mathrm{U}^{239} \rightarrow 93^{239}$ should aid in establishing the absolute beta disintegration rate of $93^{239}$.

Wahl dissolved the UNH in ether, removed the water layer, washed the ether layer twice with water, to produce UNH containing 23-minute $U^{239}$ relatively free of fission product activities; the last washing of the ether layer occurred at 11:48 a.m. He placed a small portion of this UNH on a cellophane sheet (sample no. U,n-11-1), inverted the sample and followed its decay on electroscope no 5 (step 1) beginning at 11:55 a.m. He also followed the decay on our calibrated Geiger-Mueller counter (window thickness of $4.4 \mathrm{mg}$ per sq. $\mathrm{cm}$ of mica). The sample was inverted to eliminate the effect of backscattering of the beta particles; thus they had to pass through the cellophane backing material requiring an absorption correction. The $\mathrm{U}^{239}$ decayed with a 23.2-minute half-life (yielding our best determination of this half-life) into the daughter $93^{239}$.

Using the measured beta intensities, corrected for decay, of the $\mathrm{U}^{239}\left(4.7 \times 10^{5}\right.$ counts per minute) and daughter $93^{239}(3,500$ counts per 
minute), as determined in the Geiger-Mueller counter and correcting for the absorption of the $93^{239}$ (factor of 1.9) and $\mathrm{U}^{239}$ (factor of 1.1) beta radiation in the counter window and the cellophane (using the absorption curves for these isotopes), he is able to deduce that the ratio of beta particles plus conversion electrons of $93^{239}$ to beta particles of $\mathrm{U}^{239}$ is

$$
\frac{(3,500)(1.9)(2.33)(24)(60)}{\left(4.7 \times 10^{5}\right)(1.1)(23.2)}=1.9 \pm 0.2
$$

This indicates, as we suspected, a considerable proportion of conversion electrons in the decay of $93^{239}$ leading to an overestimation of the halflife of $94^{239}$ when this is determined without correction through the use of the genetic relationship $93^{239} \rightarrow 94^{239}$. In other words, our determinations of the half-life of $93^{239}$ as $3 \times 10^{4}$ years last May, $5 \times 10^{4}$ years last August and $6 \times 10^{4}$ years in October are probably in error in the direction that the true value should be less than these values.

His measurements of the $\mathrm{U}^{239}$ beta radiation on electroscope no. 5 and the Geiger-Mueller counter enables him to calculate the efficiency of the electroscope for $\mathrm{U}^{239}$ beta radiation as 3.75 divisions per second per microcurie (step 1); this is not needed to determine the electron ratio because the Geiger-Mueller counter was used to measure the electron emission rate of both the $\mathrm{U}^{239}$ and $93^{239}$.

He also made aluminum absorption measurements of the $U^{239}$ beta particles. He found an end point of about $400 \mathrm{mg}$ per sq. cm corresponding to an upper energy limit of about $1.0 \mathrm{Mev}$ and an initial half thickness of $55 \mathrm{mg}$ per sq. $\mathrm{cm}$. Most importantly, he found no indication of conversion electrons; it appears that these are essentially absent so that this method of determining the proportion of conversion electrons in $93^{239}$ (based on the assumption they are absent in the parent $\mathrm{U}^{239}$ ) is apparently valid.

Another purpose of bombardment no. U,n-1l is to produce $93^{239}$ tracer to be used in a systematic investigation of the chemical properties of element 93. The major part of the initial aqueous extract from the UNH ether solution was used by wahl to isolate a pure $93^{239}$ fraction by the oxidation-reduction cycle using $\mathrm{BrO}_{3}{ }^{-}$as the oxidizing agent, $\mathrm{SO}_{2}$ as the reducing agent, and $\mathrm{LaF}_{3}$ as the carrier. Wahl made up a solution of this final 93 fraction (total volume about $85 \mathrm{cc}$ ) from which small aliquots can be used for the 93 tracer chemistry experiments (sample no. U,n-1l-A). In order to conduct simultaneously tracer experiments on the chemistry of element 94, he used a similar $25 \mathrm{cc}$ "stock" solution (sample 93-14-K) of our 50-year 94 alpha emitter $\left(94^{238}, 94^{236}\right.$ or $94^{235}$ ) prepared from bombardment 93-14 on March 15. The plan is to use $0.2 \mathrm{cc}$ portions of each of these element 93 and 94 "stock" solutions as sources of tracers for a series of systematic experiments on the chemical properties of elements 93 and 94 . The $93^{239}$ beta radiation will be detected with his electroscope and the 50-year 94 alpha radiation with our ionfzation chamber no. W-3. Many of the experiments will be concerned with testing which oxidizing agents are capable of oxidizing these elements from the lower (fluoride insoluble) to the upper (fluoride soluble) oxidation state; in these experiments the oxidizing agent will generally be present in approximately equal concentra- 
tions in both its oxidized and reduced forms in order to better establish the oxidation potentials for these elements.

I received a letter dated December 10, 1941 from Gregory Breit, Section S-1 in Washington, acknowledging receipt of the report on the spontaneous fission of $94^{239}$ by Kennedy and wahl sent to him on December 1, 1941. He suggested that this work should be continued because it is not certain at this stage that $94^{239}$ is better than $U^{235}$ for a nuclear weapon; it is not known whether all of the spontaneous fission in ordinary uranium is due to $U^{235}$ and thus its rate of spontaneous fission might be much lower than estimated on this basis. He said it is planned to measure the spontaneous fission rate of $\mathrm{U}^{235}$ using enriched material. In the meantime, it should be helpful to accumulate as much information as possible regarding the sponaneous fission of $94^{239}$ so as to make it possible to decide whether $94^{239}$ or $\mathrm{U}^{235}$ is the more suitable material.

Saturday, December 13, 1941

I received from Vannevar Bush a memorandum dated December 4, 1941 addressed to all persons holding appointments with the office of scientific Research and Development, National Defense Research Committee and Committee on Medical Research informing us that the Senate Special Committee Investigating the National Defense Program (Truman Committee) has called upon all Defense Agencies to furnish certain information with respect to persons holding appointments with them. Today I filled out and returned the form enclosed with the letter indicating that I am serving as an Official Investigator at the University of California while employed as a member of its teaching staff and that the University has defense contracts.

Sunday, December 14, 1941

Today Gofman began a new series of experiments in connection with the development of an efficient process for the separation of uranium from protoactinium to be used for the separation of $\mathrm{U}^{233}$ from undecayed $\mathrm{Pa}^{233}$ in sample $\mathrm{Th}+\mathrm{n}-\mathrm{I}-\mathrm{Pa}-\mathrm{I}$.

Wednesday, December 17,1941

Having experimented during the last several days and perfected methods for precipitating tracer amounts of 93 and 94 with LaF 3 in a manner where the tracers can be counted with maximum efficiency, wahl began his series of systematic investigations of the chemistry of elements 93 and 94 using as sources of tracer 0.2 cc aliquots from the "stock" solutions $U, n-11-A$ and $93-14-K$. He conducted experiments to test the oxidation of 93 and 94 by $1 \mathrm{M}$. $\mathrm{BrO}_{3}^{-}$at about $80^{\circ} \mathrm{C}$., in $0.5 \mathrm{M}$., 
$10 \mathrm{M} .$, and $15 \mathrm{M} . \mathrm{H}_{2} \mathrm{SO}_{4}$ and finds in each case that the 93 is oxidized (that is, not carried by the $\mathrm{LaF}_{3}$ ) whereas the 94 is not oxidized (that is, carried by the $\mathrm{LaF}_{3}$ ). He is now working in room 120, Gilman Hall, which has become his center of operations; this reduces the crowding in room 307 where Gofman and Friedlander are still conducting their chemical experiments.

Friday, December 19, 1941

Continuing his investigation of the chemical properties of 93 and 94 using tracer solutions $U, n-I I-A$ as a source of tracer for 93 and $93-14-K$ as a source of tracer for 94, Wahl investigated the oxidizing ability of $\mathrm{MnO}_{4}{ }^{-}$. He finds that approximately $0.1 \mathrm{M}$. $\mathrm{MnO}_{4}{ }^{-}$in approximately $2 \mathrm{M}$. $\mathrm{H}_{2} \mathrm{SO}_{4}$ solution at room temperature oxidizes 93 but not 94 .

Gofman and Stoughton completed today a bombardment of $5 \mathrm{~kg}$ of thorium nitrate (in concentrated aqueous solution) with the neutrons produced from the irradiation of beryllium with 3,000 microampere-hours of deuterons in the 60-inch cyclotron (bombardment no. $T h+n-I I)$. They commenced their procedure for the extraction of $\mathrm{Pa}^{233}$ at $8: 00 \mathrm{p} . \mathrm{m}$.

Saturday, December 20, 1941

Continuing his experiments this morning on the tracer chemical properties of elements 93 and 94 , Wahl tested the oxidizing power of a solution of $0.2 \mathrm{M} . \mathrm{Br}_{2}$ and $2 \mathrm{M} . \mathrm{H}_{2} \mathrm{SO}_{4}$ for ten minutes at room temperature and finds that neither the 93 nor the 94 is oxidized (that is, both coprecipitate with $\mathrm{LaF}_{3}$ ).

He then tested a solution of about $0.2 \mathrm{M} \cdot \mathrm{CrO}_{7}^{-2}$ and about $2 \mathrm{M}$. $\mathrm{H}_{2} \mathrm{SO}_{4}$ at room temperature for ten minutes and finds that 93 is oxidized while 94 is not.

In the afternoon he tested the oxidation by $\mathrm{BrO}_{3}^{-}$in $2 \mathrm{M}^{-} \mathrm{H}_{2} \mathrm{SO}_{4}$ for ten minutes at room temperature and finds that 93 is oxidized and 94 is not. This apparently is a reliable method for separating 93 from 94 , and due to its general applicability deserves a name-we refer to it as the "cold bromate method."

Next he tested the oxidizing power of $0.2 \mathrm{M}$. $\mathrm{MnO}_{4}{ }^{-}$in $2 \mathrm{M} \mathrm{H}_{2} \mathrm{SO}_{4}$ for ten minutes at room temperature and finds that the 93 is oxidized and the 94 is not.

Arthur $\mathrm{H}$. Compton wrote a memorandum addressed to Vannevar Bush, James B. Conant and Lyman J. Briggs outlining a theoretical and experimental program to be centered at the University of Chicago and carried on with the cooperation of the groups at Columbia, Princeton and Berkeley for the production of $94^{239}$, for use in a nuclear explosive, through the 
operation of a chain reaction with natural uranium. Spurred on by Pearl Harbor, he suggested a speeded up time schedule of obtaining knowledge of the conditions for a chain reaction by June 1, 1942, production of a chain reaction by October 1, 1942, a pilot plant for use of the chain reaction to produce 94 by October 1, 1943, and the production of usable quantities of 94 by December 31, 1944.

Sunday, December 21, 1941

Continuing his investigation of the tracer chemistry of 93 and 94 , Wahl used a solution $0.1 \mathrm{M} . \mathrm{S}_{2} \mathrm{O}_{8}^{-2}, 10^{-2} \mathrm{M}$. Agt and $1 \mathrm{M} . \mathrm{H}_{2} \mathrm{SO}_{4}$ for ten minutes at room temperature and finds complete oxidiation of 93 but only partial oxidation of 94 .

Monday, December 22, 1941

Gofman took one of the later electrolytically deposited plates of $\mathrm{Pa}^{233}$ (sample $\mathrm{Th}+\mathrm{n}-\mathrm{I}-\mathrm{Pa}-\mathrm{IV}$ from bombardment $\mathrm{Th}+\mathrm{n}-\mathrm{I}$ which was prepared last month) in order to practice the recovery of daughter $\mathrm{U}^{233}$. By comparison of the intensity of $\mathrm{Pa}^{23}$ beta radiation in this sample with that of sample Th+n-I-Pa-III and the growth of daughter $\mathrm{U}^{23}{ }^{3}$ in the latter, we can estimate the number of $U^{233}$ alphas that should be present in this sample as corresponding to a decay rate of 100 per minute. He scraped the sample from the copper backing plate, dissolved it in acid, removed the dissolved copper by precipitation of CuS, and then removed the undecayed $\mathrm{Pa}^{233}$ by numerous precipitations with zirconium phosphate. The final filtrate from these was then evaporated to a small volume and subjected to electrolysis beginning at $6: 15 \mathrm{p} . \mathrm{m}$.

Today I sent letters to E. I. du Pont de Nemours, Wilmington, Delaware, General Chemical Company, New York City, S. W. Shattuck Chemical Company, Denver, Colorado, Merck \& Co., Inc., Rahway, New Jersey, and Eimer and Amend, New York City inquiring about the availability of large amounts of UNH and about the availability of mother liquors remaining after recrystallization of UNH. I have in mind starting a program for the recovery of microgram amounts of $\mathrm{U}^{234}$ through the extraction of the intermediate 24.5-day $\mathrm{UX}_{1}$ which would be allowed to decay to the daughter $\mathrm{U}^{234}$. The daughter $\mathrm{U}^{234}$ would then be separated from the undecayed $\mathrm{UX}_{1}$ in a form suitable to measure its slow and fast neutron fission cross sections and its rate of decay by spontaneous fission. 
Gofman and Stoughton completed the isolation of the $\mathrm{Pa}^{23}$ from bombardment $T h+n-I I$ by the chemical procedure started last Friday. They used a procedure like that used on sample $\mathrm{Th}+\mathrm{n}-\mathrm{I}-\mathrm{Pa}-\mathrm{I}$. The $\mathrm{Pa}^{2.33}$ was extracted from the dissolved $5 \mathrm{~kg}$ of thorium nitrate by coprecipitation with manganese dioxide, followed by the reduction of carrier by several alternate precipitations of zirconium phosphate and manganese dioxide. The last small zirconium phosphate precipitate containing the $\mathrm{Pa}^{233}$ was dissolved and the $\mathrm{Pa}^{233}$ separated by electrolysis onto copper, leading to a $\mathrm{Pa}^{233}$ sample with beta radiation of about 40 millicuries as determined very roughly by measurement with the ionization chamber FP-54 setup. This $\mathrm{Pa}^{23}$ sample will be divided into a number of fractions and used for the calibration of our ionization chamber FP-54 setup and Lauritsen electroscopes for the absolute measurement of the beta radiation from $\mathrm{Pa}^{233}$.

Gofman completed the electrolysis started yesterday and measured the resultant alpha activity on our alpha ionization chamber G-2. He found an intensity of about 15 alphas per minute and although the yield is lower than expected, this sample is notable in that it represents an actual isolation of the long-sought daughter $\mathrm{U}^{233}$ from its parent $\mathrm{Pa}^{23}$.

Saturday, December 27, 1941

I received a letter dated December 24, 1941 from Dr. Lyman J. Briggs in reply to my letter of November 8,1941 indicating that my further work on the search for elements 93 and 94 in ores will be funded as part of the large contract which is being put into operation under the direction of Professor Lawrence.

Monday, December 29, 1941

During the last few months Wahl, Friedlander, Leitz and English have undertaken a program to calibrate our Geiger-Mueller counters, for use to improve the calibration of our ionization chamber FP-54 setup, and Lauritsen electroscopes, to establish their efficiency for the detection of beta radiation. The aim has been to make it possible to better determine the intensity of beta activity in our samples in an absolute sense. They have calibrated our Geiger-Mueller counters through the use of weighed $U$ in equilibrium with $U \mathrm{UX}_{1}-\mathrm{UX}_{2}$, aliquots of $\mathrm{UX}_{1}$ separated quantitatively from a known amount of UNH, and a sample of Radium $D$ in equilibrium with Radium $E$ (whose beta particles were counted with the Geiger-Mueller counters) and Radium $F$ (whose alpha particles were counted with our ionization chamberlinear amplifier setup, W-3, of known efficiency). All sources were mounted on thin metal foils (usually $A 1$ ) and the beta counting was done with the source side down to eliminate backscattering; appropriate corrections were made for the absorbing power of the metal foil and mica window of the counter. With this procedure they have established the counting efficiency of our exceptionally good Geiger-Mueller (belljar type) counters no. z-32 and $z-33$, built this month, as $0.29 \pm 0.02$ for radioactive isotopes whose 
beta particles have negligible absorption in the mica window (thickness approximately $3 \mathrm{mg} / \mathrm{cm}^{2}$ ); for samples with low energy beta particles, corrections must be made for absorption by the mica window. Each of the counters $\mathrm{z}-32$ and $\mathrm{z}-33$ is in operation with its individual amplifier, scale of eight recording circuit with cenco counter.

The availability of absolute beta counting makes it possible to better calibrate our ionization chamber FP-54 setup in an absolute sense to determine the radiation intensities of samples of $93^{239}, \mathrm{~Pa}^{233}$ and so forth measured on this instrument; similarly, absolute calibration of our electroscopes can be made.

They also prepared our Radium D standard (standard RaE St. I), whose Radium $E$ beta particles are measured, and our uranium standard UX $\left.{ }_{2}-S t .-4\right)$, whose $\mathrm{UX}_{2}$ beta particles are measured, which are being used as checks on the sensitivity of our ionization chamber FP-54 and Lauritsen electroscopes during series of measurements that take place over a long time (such as the beta decay of samples $T h+n-I-P a-I I I$ and $-I I)$.

I received a letter from Gregory Breit in Washington dated December 26, 1941 saying that he is moving as of January 2, 1942 to the University of Chicago to work on the large element 94 project that is starting there under Professor Arthur H. Compton.

Tuesday, December 30, 1941

Wahl bombarded $135 \mathrm{gm}$ of UNH from 8:00 to 8:30 p.m. with the neutrons produced by the bombardment of beryllium with 50 microampere-hours of deuterons in the 60-inch cyclotron (bombardment no. $\mathrm{U}, \mathrm{n}-12$ ). One purpose of this bombardment is to measure again the decay of 23.2-minute $\mathrm{U}^{239}$ into 2.3-day $93^{2.39}$ in order to establish through this genetic relationship the number of conversion electrons emitted in the decay of $93^{239}$, on the assumption that $U^{239}$ has essentially no conversion electrons and its measured particle radiation is all due to beta particles. This is a prelude to determining a better value of the half-life of $94^{239}$ through the decay chain $\mathrm{U}^{239} \rightarrow 93^{239} \rightarrow 94^{239}$.

Wahl dissolved the UNH in ether, removed the aqueous extract, washed the ether layer twice with $\mathrm{H}_{2} \mathrm{O}$, then measured the decay of the $\mathrm{U}^{2} 39$ in a portion of the uranium from the ether fraction (sample no. U, n-12-1) mounted on cellophane. He plans to follow the decay of this $\mathrm{U}^{239}$ using our GeigerMueller counter no. z-33 (window thickness of $3.0 \mathrm{mg}$ per sq. cm of mica) on step 1 until the decay curve turns over to that of the daughter $93^{239}$ and thus will be able to establish the intensities of both the $v^{239}$ and $93^{239}$ as measured on this same instrument. The only corrections required are to allow for the absorption of the $\mathrm{U}^{239}$ and $93^{239}$ beta particles in the window of the Geiger-Mueller counter. This is easily done with the help of the absorption curves for the beta radiation of these isotopes. 
He measured the absorption in aluminum of the $\mathrm{U}^{239}$ beta radiation of sample U,n-12-1 and of another portion (sample no. U,n-12-2) and found an end point of about $700 \mathrm{mg} \mathrm{Al}$ per sq. $\mathrm{cm}$ corresponding to an upper energy limit for the beta particles of about $1.6 \mathrm{Mev}$; most important, he found no indication of conversion electrons, showing that all the electrons from $\mathrm{U}^{239}$ are beta particles and can be used to determine the number of daughter $93^{239}$ and granddaughter $94^{239}$ nuclei.

He also plans to isolate a sample of pure $93^{239}$ from this bombardment to be used in further studies of the chemical properties of element 93 .

Wednesday, December 31,1941

From Wahl's measurement of the decay of the $U^{239}$ isolated yesterday and his intensity of the beta radiation from the daughter $93^{239}$ measured early this morning, we can calculate the ratio of beta particles and conversion electrons from $93^{239}$ to the beta particles from $\mathrm{U}^{239}$. Using the beta intensities, corrected for decay, of $\mathrm{U}^{239}\left(4.2 \times 10^{4}\right.$ counts per minute) and $93^{239}$ (480 counts per minute), and the corrections for absorbtion in the mica window of the Geiger-Mueller counter, a factor of 1.04 for $U^{239}$ and 1.36 for $93^{239}$, we find for this ratio

$$
\frac{(480)(1.36)(2.33)(24)(60)}{\left(4.2 \times 10^{4}\right)(1.04)(23.2)}=2.1 \pm 0.3 .
$$

Combining this with the value $(1.9 \pm 0.2)$ obtained on December 12 , we have an average value of $2.0 \pm 0.3$ for the ratio of the beta particles plus conversion electrons of $93^{239}$ to the beta particles of $\mathrm{U}^{239}$. This enters as a correction factor in the determination of the half-life of $94^{239}$ through use of the genetic relationship $93^{239} \rightarrow 94^{239}$.

Thursday, January 1,1942

Wahl performed an oxidation-reduction cycle using $\mathrm{BrO}_{3}^{-}$as oxidizing agent, $\mathrm{SO}_{2}$ as reducing agent, and $\mathrm{LaF}_{3}$ as carrier to isolate a $93^{239}$

fraction (U,n-12-A) from last Tuesday's uranium-neutron bombardment for use as a source of tracer activity in his planned experiments to continue his study of the chemistry of element 93.

Friday, January 2, 1942

A greatly expanded effort is getting underway at many places on the overall project of the preparation of fissionable material for use in a nuclear weapon. This now has a high national priority and a large contract ( $\$ 405,000$ for six months) in the name of Lawrence is going into 
effect here at the University of California, Berkeley. This covers all of the work that I am doing, other than a couple of my specific contracts, as well as Lawrence's ambitious program for the separation of $\mathrm{U}^{235}$ by the electromagnetic process, to which Kennedy and Segrè are shifting their efforts. Similarly, a large program is getting underway at the University of Chicago under Arthur H. Compton concerned with the production of the isotope $94^{239}$ from the nuclear chain reaction with natural uranium; this will be the center for this research program which has been given the code name Metallurgical Project, with the main component of this, at the University of Chicago, to be known as the Metallurgical Laboratory. The overall organization of section S-1 has been changed. In Section S-1, still under the chairmanship of Dr. Lyman J. Briggs and remaining in the office of Scientific Research and Development, the responsibility for the scientific aspect is divided between the Program Chiefs, E. O. Lawrence, A. H. Compton and H. C. Urey, working with Dr. J. B. Conant, Chairman of the National Defense Research Committee. In addition, also with OSRD, there will be a Planning Board of distinguished chemical engineers with Mr. E. V. Murphree of the Standard Oil Development Company of New York as its chief.

I am assuming leave status from my position as Assistant Professor of Chemistry in order to devote full time to this high priority research program. My group is being substantially expanded in order to broaden the scope of my work. My graduate students, Arthur wahl (who has essentially completed his graduate work), Jack Gofman, Spofford English and John Hamaker, are continuing on their programs: Wahl on the investigation of the chemical properties of elements 93 and 94 , the identification of $93^{237}$ and the measurement of the radioactive and fission properties of $94^{239}$ and $93^{237}$ and the radioactive properties of the 50-year 94 and its 2.0 -day 93 parent; English is collaborating with wahl on the measurement of the radioactive and fission properties of the two isotopes of element 94; Gofman is continuing his work on the search for $\mathrm{U}^{233}$ and the measurement of its radioactive and fission properties in collaboration with Dr. Raymond $\mathrm{w}$. Stoughton (who is working as a postdoctorate under my contract OEMsr-206 supporting this work); Hamaker is working to set up the apparatus for eventual ultra microchemical investigations of the chemical properties of $94^{239}$. Morris Perlman is continuing his work on the search for elements 93 and 94 in uranium ores. In addition, Robert B. Duffield, a graduate student working with Professor Melvin Calvin, is shifting to participation in our program in view of its urgency.

I have two undergraduate students starting work on a new project. As the result of conversations with Dean Wendell M. Latimer, arrangements have been made for Rene J. Prestwood to take 15 units of undergraduate research work under my direction for completion of his work for the B.S. degree in chemistry; he will be taking 12 units of undergraduate laboratory research work, chemistry course $180 \mathrm{H}$, and 3 units of library research work, chemistry course 199. Also continuing his work with me as an undergraduate research student this semester, taking chemistry courses $180 \mathrm{H}$ and 199, is Norman A. Bonner; in his work with me last semester he investigated methods for separating rare earths, thorium and uranium from each other. I am starting them on the new project of isolating $U^{234}$, for the purpose of measuring its neutron and spontaneous fission properties, from large amounts 
of uranium. The procedure will be to isolate the 24-day $\mathrm{Th}^{234}$ isotope, $U_{1}$, from large amounts of $U N H$, then let the $U X_{1}$ decay to $U^{234}$ which is in turn to be separated from the undecayed $\mathrm{UX}_{1}$. It was with this purpose in mind that I had Bonner work on the separation methods last semester.

Also joining me at this time are some Ph.D. scientists in addition to Dr. Stoughton. First and foremost is Dr. Isadore Perlman, a fellow student of mine from UCLA who obtained his Ph.D. in the Department of Physiology here at Berkeley where he has been doing postdoctorate work. He is an outstanding scientist with a thorough understanding of chemistry and I have in mind that he will soon be a great help in overall direction in the work of the group. Another Ph.D. scientist is Beppino J. Fontana, who recently received his Ph.D. in organic chemistry here at Berkeley. I am asking Perlman and Fontana to start by working with Prestwood and Bonner on the preparation of samples of $\mathrm{U}^{234}$.

We also have our energetic technician, Gayle E. Adams, continuing to help us as a jack-of-all-trades.

Isadore Perlman, Morris Perlman, Fontana, Wahl, Kennedy and I will be on the payroll of the new large Lawrence contract, Stoughton will be continuing under my contract OEMsr-206, and the graduate and undergraduate students are doing their work as part of their academic program.

The size of our group has necessitated our expansion into a number of additional rooms in Gilman Hall. Kennedy and I have our office in room 303, which also houses most of our counting equipment. Duffield and Hamaker are in room 305, Friedlander and Gofman in room 307, Stoughton and Morris Perlman in room 311. We are also going to occupy room 315, but this is now largely a storage room and has not been used recently as a chemistry laboratory. These rooms are all on the west side of the corridor on the third floor of Gilman Hall. Wahl has shifted to room 120 of Gilman Hall because of our expanding demand for space. He is using this room for his experiments on the study of the chemistry of 94 and 93 as well as for his counting experiments.

Saturday, January 3,1942

Prestwood and Adams worked on moving the equipment stored in room 315 to room 13 in the basement for storage there and started the task of getting room 315 in shape for use as a chemistry laboratory.

Monday, January 5, 1942

Prestwood and Adams worked all day today and yesterday to get the hood in working order and to make the other necessary changes in room 315. 
Today Isodore Perlman, Fontana, Prestwood and Bonner started the process of extracting $\mathrm{UX}_{1}$ from our 90 pounds of Baker and Adamson UNH purchased from the General Chemical Company last November. We labelled this Batch I and intend that this be the first of a series of such extractions from additional large amounts of UNH that we have ordered from the General Chemical Company and du Pont Company. These men are working in room 301, Gilman Hall, which we have just acquired in our expansion program and which is equipped with a fine porch for outdoor work convenient for conducting a number of the steps in our proposed chemical procedure. The contemplated procedure consists of treatment of large amounts of uranyl nitrate hexahydrate $\left(\mathrm{UO}_{2}\left(\mathrm{NO}_{3}\right)_{2} \cdot 6 \mathrm{H}_{2} \mathrm{O}\right.$, UNH) by a procedure that has been largely worked out last year in our chemical isolation of 94 and 93 from pound quantities of neutron-bombarded UNH. The basis for this procedure is the extraction of the bulk of the UNH into ether, in which process part of the water of crystallization separates and this contains essentially all of the $\mathrm{JX}_{1}$ together with the inorganic impurities such as $\mathrm{NaNO}_{3}$ (or, in the case of the neutron bombarded UNH, all of the 93 and 94 and fission products). The aqueous fraction contains only about $10 \%$ of the UNH and this can be further reduced tenfold by crystallizing it and extracting it again with ethex. This process can be repeated a third and fourth time if necessary. The final aqueous phase contains the $\mathrm{UX}_{1}$ and it is contemplated to isolate this by coprecipitating it with a mixture of $\mathrm{LaF}_{3}$ containing a little $\mathrm{CeF}_{4}$ and reprecipitating this several times. The $\mathrm{UX}_{1}$ will then be further concentrated by coprecipitation with ceric iodate from acid solution in a procedure that eliminates the larger quantity of $\mathrm{La}^{+++}$carrier by leaving it in solution. If any thorium is present, it, of course, will also be precipitated as thorium iodate along with the ceric iodate. The cerium can be eliminated by reduction to $\mathrm{Ce}^{+3}$ followed by precipitation of thorium iodate from acid solution leaving the $\mathrm{Ce}^{+3}$ in solution. The $\mathrm{Th}^{+}{ }^{4}$ (or $\mathrm{Ce}^{+4}$ if $\mathrm{Th}$ is not present) will then be precipitated, and reprecipitated several times, as $\mathrm{ThF}_{4}$ (in the presence of $\mathrm{HNO}_{3}$ to insure that $\mathrm{U}$ is removed as soluble $\mathrm{UO}_{2}{ }^{++}$). After solution of the final $\mathrm{ThF}_{4}$ (or $\mathrm{CeF}_{4}$ ) in acid, this UX $x_{1}$-containing fraction will then be allowed to decay, after which the daughter $U^{234}$ will be isolated by removing the cerium (and thorium, if present) by fluoride precipitation. The $\mathrm{U}^{2.34}$ will then be electroplated onto a platinum foil using a modification of the process worked out by Gofman and Stoughton for the isolation of $\mathrm{U}^{233}$. The last two steps, that is the separation of the $\mathrm{Ce}^{++++}$(and thorium) from the $\mathrm{La}^{+++}$, and the final isolation of the $\mathrm{U}^{234}$ are yet to be worked out in detail.

There are about 70 microcuries of $\mathrm{UX}_{1}$ in equilibrium with the uranium present in one pound of UNH and 23 millicuries is the equivalent radiation of 1 microgram of $\mathrm{UX}_{1}$ which, of course, will decay to 1 microgram of $\mathrm{U}^{234}$.

Using beta emitting $93^{239}$ sample no. U,n-12-A, wahl prepared this evening a "stock"solution of element 93 tracer which he will use together with 50-year 94 alpha-emitting stock solution 93-14-K in his continuing study of the chemistry of elements 93 and 94 .

Using 0.2 cc aliquots of these "stock" solutions of element 93 and 94 tracers, wahl performed an oxidation experiment using $0.2 \mathrm{M} \mathrm{Br}$, in 
2. M. $\mathrm{H}_{2} \mathrm{SO}_{4}$; the potential reaction was allowed to proceed for 25 minutes at room temperature. The $\mathrm{LaF}_{3}$ precipitate carried both the 93 and 94 tracers, indicating that neither element is oxidized. He then performed a similar experiment except that the oxidizing agent was changed to 0.17 M. $\mathrm{Cr}_{2} \mathrm{O}_{7}^{-2}$. In this case, the 93 was oxidized (that is, the 93 tracer was not carried by the $\mathrm{LaF}_{3}$ ), while the 94 was not oxidized (that is, the 94 tracer was carried by $\mathrm{LaF}_{3}$ ).

Wednesday, January 7,1942

Today Isadore Perlman, Fontana, Prestwood and Bonner finished the first ether extraction of Batch $I$.

Thursday, January 8,1942

Gofman dissolved the $\mathrm{Pa}^{233}$ of sample $\mathrm{Th}+\mathrm{n}-\mathrm{I}-\mathrm{Pa}-\mathrm{IV}$ (prepared on November 19) in acid in order to make a practice run to recover the daughter $\mathrm{U}^{233}$. He removed the dissolved copper by precipitation of copper sulfide, then precipitated a number of zirconium phosphate precipitates to remove the undecayed $\mathrm{Pa}^{233}$, finally added acetic acid and electrolyzed the solution to recover the $U^{233}$. He calculates from the estimated amount of $\mathrm{U}^{23}$ in the sample at this date that he achieved a recovery of $75 \%$. This is a pretty good test of the procedure to separate uranium from protoactinium which is under development in order to recover our large sample of $\mathrm{U}^{233}$ from sample $\mathrm{Th}+\mathrm{n}-\mathrm{I}-\mathrm{Pa}-\mathrm{I}$. The $\mathrm{Pa}^{233}$ content could be estimated by comparison with the growth of $U^{23}$ alpha activity into sample Th+n-I-Pa-II as of this date using the ratio of beta intensities of samples Th+n-I-Pa-IV and -II.

This is also notable in that it represents an actual isolation of the daughter $\mathrm{U}^{233}$ from its parent $\mathrm{Pa}^{233}$.

Today stoughton completed his measurements on the backscattering of $\mathrm{Pa}^{23}$ beta particles from copper using part of the $\mathrm{Pa}^{233}$ sample isolated on December 23 from bombardment $T h+n-I I$. The purpose is to calibrate our ionization chamber FP-54 setup so that we can convert our readings on samples $\mathrm{Th}+\mathrm{n}-\mathrm{I}-\mathrm{Pa}-\mathrm{II}$ and -III to absolute intensities of $\mathrm{Pa}^{23}$ beta radiation. His measurements show that a sample of $\mathrm{Pa}^{233}$ mounted on copper leads to 498 greater deflection on the recording system than does an identical sample mounted on cellophane; this corresponds to a correction factor of $1 / 1.49=0.67$. The small effect of backscattering from cellophane can also be corrected for by comparing the Geiger-Mueller counting rate of a $\mathrm{Pa}^{233}$ sample mounted on cellophane in the upright position but covered by an identical sheet of cellophane with that of the sample in the inverted position. The average of several such determinations of this comparison for $\mathrm{Pa}^{23}$ radiation has shown that the counting rate for the covered sample measured upright is 1.03 times that of the counting rate for the uncovered sample measured inverted. Thus, there is a $3 \%$ 
increase due to backscattering due to cellophane, and the correction factor is $1 / 1.03=0.97$.

Friday, January 9, 1942

This evening Wahl performed an oxidation experiment using the "stock" 93 and 94 tracer solutions with $0.2 \mathrm{M}$. $\mathrm{MnO}_{4}^{-}$in $2 \mathrm{M}^{-} \mathrm{H}_{2} \mathrm{SO}_{4}$ at $80^{\circ} \mathrm{C}$. with the result that both the 93 and 94 are oxidized. He also performed an experiment with $10 \mathrm{M}$. $\mathrm{Ce}^{+{ }^{l_{4}}}$ in $5 \mathrm{M}$. $\mathrm{H}_{2} \mathrm{SO}_{4}$ with the result that the 93 was partially oxidized and the 94 was not oxidized.

Isadore Perlman, Fontana, Prestwood and Bonner recrystallized the UNH from the first ether extraction of Batch I and prepared it for the second ether extraction and the subsequent isolation of the UX $\mathrm{U}_{1}$ fraction to be carried out by Fontana and Bonner. This recrystallization process is rather tricky because it is necessary to come out with exactly $6 \mathrm{H}_{2} \mathrm{O}$ of crystallization. The fellows have found that it is necessary to evaporate the UNH to a density which is almost precisely $2.4 \mathrm{gm}$ per cc.

Stoughton completed today a calibration of our ionization chamber F'P-54 electrometer for the beta radiation of $\mathrm{Pa}^{233}$ using samples isolated from bombardment $\mathrm{Th}+\mathrm{n}-\mathrm{I}-\mathrm{Pa}-\mathrm{II}$ on December 23. He measured the absolute electron intensity of one sample of $\mathrm{Pa}^{233}$ mounted on cellophane with our Geiger-Mueller counter no. z-33, and with Lauritsen electroscope no. 9 with the sample on the second step. Using the known geometrical factor for the counter (29\%) and correcting for the absorption of $\mathrm{Pa}^{23}$ beta radiation in the window of the counter $(3.0 \mathrm{mg}$ per sq. $\mathrm{cm}$ mica), he calculates the sensitivity of our electroscope no. 9 as 2.66 divisions per second per microcurie. Then he measured the radiation of another sample of $\mathrm{Pa}^{233}$ of higher beta intensity with both the electroscope no. 9 and our ionization chamber FP-54 setup (with the sample on step 2). Converting his results to our "standard conditions" (resistance and shunt settings R5S5), he finds a $0.061 \mathrm{~mm}$ galvanometer deflection per microcurie of $\mathrm{Pa}^{2} 33$ electron radiation. By "microcurie" here, we mean total electron radiation, conversion electrons as well as nuclear beta particles.

Today we received another 200 pounds of UNH bearing the Baker and Adamson label from the General Chemical Company in response to our purchase order (Batch II).

Saturday, January 10, 1942

This morning wahl performed an experiment using the 93 and 94 tracer solutions with $0.2 \mathrm{M} \mathrm{Br} 2$ in $2 \mathrm{M} . \mathrm{H}_{2} \mathrm{SO}_{4}$ as oxidizing agent for two hours at $0^{\circ}$ C. with the result that neither the 93 nor the 94 is oxidized.

Today chemical separations were started on $300 \mathrm{gm}$ of UNH which have been bombarded intermittently from 6:00 p.m. January 5 to midnight, 
January 9, with fast neutrons produced by the irradiation of beryllium with $16 \mathrm{Mev}$ deuterons in the 60-inch cyclotron (bombardment no. U,n-13). The UNH was placed close to the beryllium target with no intervening paraffin because one of the aims is to produce $U^{237}$ in order to calibrate our Lauritsen electroscope no. 5 for its efficiency in measuring $U^{237}$ beta radiation. This relates to the experiment conducted on March 31, 1941 and April 2-3, 1941 (bombardment no. 93-15) performed to determine the yield of $U^{237}$, for which the calibration of electroscope no. 5 is needed. The aim here is to measure the intensity of $\mathrm{U}^{237}$ radiation in a weighed portion of UNH, from which the other transmutation products have been chemically separated, using electroscope no. 5, and then determine the absolute disintegration rate of other small weighed aliquot portions using our calibrated thin-windowed Geiger-Mueller counter no. z-33.

S. G. English began the chemical separation procedure by dissolving the UNH in ether, separating the aqueous layer, washing the uraniumcontaining ether with numerous portions of $\mathrm{H}_{2} \mathrm{O}$, then extracting the main portion of $\mathrm{UNH}$ back into $\mathrm{H}_{2} \mathrm{O}$ and removing the remaining ether by evaporation. Wahl carried on the separation by recrystallizing this $\mathrm{U}^{237}$-containing UNH a couple of times.

Because our UNH Batch II is too lumpy for a successful ether extraction, Isadore Perlman and Prestwood started grinding it down to fine crystals using the ball mill in room 15 of Hilgard Hall in order to reduce the material to a size where it will go through a 20 mesh screen, which they find is suitable for carrying on the ether extraction.

Sunday, January 11,1942

Wahl mounted on cardboard $100 \mathrm{mg}$ (sample $\mathrm{U}, \mathrm{n}-13-\mathrm{C}-1$ ) and $16.6 \mathrm{mg}$ (sample $\mathrm{U}, \mathrm{n}-13-\mathrm{C}-2$ ) of finely ground $\mathrm{U}^{237}$-containing UNH from yesterday's bombardment. His measurements on step 1 with electroscope no. 5 show intensities of 0.043 and 0.0125 divisions per second. (The $100 \mathrm{mg}$ and $16.6 \mathrm{mg}$ are meant to correspond closely, including identical cellophane covering, with the $100 \mathrm{mg}$ sample 93-15-1 and $16 \mathrm{mg}$ sample 93-15-2 upon which $U^{237}$ yield measurements were made on April 3, 1941.)

He mounted two small additional portions of the $\mathrm{U}^{237}$-containing UNH, approximately $0.3 \mathrm{mg} U$ (sample no. $U, \mathrm{n}-13-\mathrm{C}-3$ ) on cellophane, and $0.2 \mathrm{mg}$ $\mathrm{U}$ (sample no. U,n-13-C-4) deposited by electrolysis on platinum, for absolute beta counting on Geiger-Mueller counter no. Z-33; he determined the weight of the uranium in these samples by measuring carefully the rate of emission of alpha particles using our alpha counter (ionization chamber) no. $\mathrm{W}-3$. These measurements indicate a weight of $0.280 \pm 0.015 \mathrm{mg}$ of $\mathrm{U}$ in sample $\mathrm{U}, \mathrm{n}-13-\mathrm{C}-3$ and $0.200 \pm 0.010 \mathrm{mg}$ in sample $\mathrm{U}, \mathrm{n}-13-\mathrm{C}-4$. The average counting rate of the two samples, as measured on Geiger counter no. z-33, translated to absolute beta disintegration rate by correcting for the geometrical efficiency of the counting arrangement, correcting for the backscattering from $\mathrm{Pt}$ of sample no. U,n-13-C-4 (done empirically by making measurements with $P t$ placed under sample no. U,n-13-C-3), amounts to 2150 beta disintegrations per minute per mg of $U$ or 1020 beta disintegrations per minute per mg of UNH. 
Thus we can calculate the sensitivity of our electroscope no. 5 for the beta radiation of $U^{237}$ under the conditions of our measurements as:

$$
\begin{aligned}
\frac{(0.043)(60)\left(3.7 \cdot 10^{4}\right)}{(1020)(100)}= & \begin{array}{l}
0.94 \text { divisions per second per micro- } \\
\text { curie for the } 100 \mathrm{mg} \text { sample }(U, \mathrm{n}-13-\mathrm{C}-1),
\end{array} \\
& \text { and } \\
\frac{(0.0125)(60)\left(3.7 \cdot 10^{4}\right)}{(1020)(16.6)}= & \begin{array}{l}
1.64 \text { divisions per second per micro- } \\
\text { curie for the } 16.6 \mathrm{mg} \text { sample }(\mathrm{U}, \mathrm{n}-13-\mathrm{C}-2) .
\end{array}
\end{aligned}
$$

Monday, January 12,1942

This afternoon wahl performed another oxidation experiment with $0.2 \mathrm{M}$. $\mathrm{MnO}_{4}{ }^{-}$in $2 \mathrm{M}$. $\mathrm{H}_{2} \mathrm{SO}_{4}$ using the "stock" 93 and 94 tracers. The reaction took place at $80^{\circ}$ C. with the result that both 93 and 94 are oxidized.

Gofman took a sample of $\mathrm{U}^{233}$ and added a couple of micrograms of natural uranium to test further the separation of uranium from protoactinium. From acid solution he precipitated the dissolved copper as copper sulfide, made a number of precipitations of zirconium phosphate to remove the $\mathrm{Pa}^{233}$, added acetic acid to the remaining solution, and electrolyzed to obtain a yield of 75\%. This indicates that there is no great difference in yield between microgram and submicrogram amounts of uranium in their evolving procedure.

Another undergraduate student, Charles E. Auerbach, started working with me as a chemistry $180 \mathrm{H}$ student; I am assigning him to the $\mathrm{UX}_{1}$ extraction problem.

Kennedy, working with Segrè, is spending more and more of his time on measurements of the yield and properties of enrighed $\mathrm{U}^{235}$ samples prepared in connection with Lawrence's program of the use of the 37-inch cyclotron magnet as a mass spectrograph for the enrichment of $U^{235}$.

Tuesday, January 13,1942

This afternoon wahl performed an oxidation experiment using $0.2 \mathrm{M}$. $\mathrm{Br}_{2}$ in $2 \mathrm{M}$. $\mathrm{H}_{2} \mathrm{SO}_{4}$ for 15 minutes at $80^{\circ}$ C. with the result that neither 93 nor 94 is oxidized. He also performed an experiment with $0.2 \mathrm{M}$. $\mathrm{BrO}_{3}{ }^{-}$ in $2 \mathrm{M} \mathrm{H}_{2} \mathrm{SO}_{4}$ at $80^{\circ}$ C. with the result that 93 is quantitatively oxidized whereas 94 is not oxidized.

Wednesday, January 14, 1942

Isadore Perlman and Prestwood finished grinding the 200 pounds of UNH of Batch II and making a practice run of 1 pound through the ether extraction process. They find there is a sludge in the water layer which 
apparently doesn't carry much of the $\mathrm{UX}_{1}$, but we'll have to contend with this in the subsequent work on this batch.

Friday, January 16,1942

Fontana and Bonner have completed their isolation of the $\mathrm{UX}_{1}$ fraction from Batch I started on January 6. They encountered sludge material in the acueous phases but found that treatment with acid extracted the $U_{1}$ in this material and these extracts could be combined with the aqueous phase from which the sludge had been removed by centrifugation. However, this was not much more than a practice run and much work needs to be done to perfect the isolation procedure.

Saturday, January 17,1942

Gofman took some of the $\mathrm{Pa}^{233}$ isolated on December 23 from bombardment $T h+n-I I$ in order to prepare a $U^{233}$ stock solution for use in subsequent experiments. He removed the $\mathrm{Pa}^{23}$ very completely by repeated precipitations with zirconium phosphate. This stock solution now contains a concentration of $\mathrm{U}^{233^{3}}$ corresponding to about 143 alpha counts per minute (on ionization chamber $\mathrm{W}-3$ ) per $\mathrm{cc}$, or about 300 alpha disintegrations per minute per $\mathrm{CC}$.

I received a letter dated January 16, 1942 from Joe Hamilton, who is in New York this week, indicating that he has expedited the shipment of the $300 \mathrm{mg}$ Ra-Be neutron source (which we are renting from Canadian Radium and Uranium Corporation) and it is now on its way to Berkeley. He warned me that it is not very well sealed and thus may leak radon. He said that the glass container and lead case may both be badly contaminated with radioactivity. We hope to use this source for our measurements of fissionability of heavy isotopes.

I wrote to Hamilton at the Commodore Hotel in New York City to indicate that our Purchasing Department is today sending an order for the rental of the $300 \mathrm{mg}$ Ra-Be neutron source to the Canadian Radium and uranium Corporation in New York. They are also sending out a request for bids on the rental of a $1 \mathrm{gm}$ Ra-Be neutron source.

Monday, January 19,1942

Gofman and Stoughton have completed their experiments for separating uranium from protoactinium and have established a procedure for separating the daughter $\mathrm{U}^{233}$ from undecayed $\mathrm{Pa}^{233}$ in sample no. Th+n-I-Pa-I. The thin film containing the $\mathrm{Pa}^{233}$ and $\mathrm{U}^{233}$ will be dissolved in acid, the small amount of copper dissolved from the backing plate will be removed by $\mathrm{H}_{2} \mathrm{~S}$ precipitation from acid solution. From this solution zirconium phosphate 
will be precipitated; a large number of zirconium phosphate precipitations will be performed in order to remove more than 99.98 of the undecayed $\mathrm{Pa}^{233}$. The volume of this solution will then be reduced to about $5 \mathrm{cc}$ and after the adjustment of the acidity, including the addition of acetic acid, the electrolysis onto a platinum plate will be carried out. By this procedure Gofman and Stoughton have demonstrated that more than $90 \%$ of the uxanium is plated out in a well-adhering film, probably platinum from the electrodes, of entirely negligible weight (about $0.2 \mathrm{mg} / \mathrm{cm}^{2}$ ).

Today Gofman used the $\mathrm{U}^{233}$ tracer prepared last saturday to make a careful study of the electrolytic deposition of $U$. He used what he now thinks is the optimum concentration of acetic acid and the best conditions for electrolysis, and succeeded in obtaining a recovery of about $97 \%$.

He then went on to test his procedure in practice by beginning with a portion of the $\mathrm{Pa}^{23}-\mathrm{U}^{233}$ plate on copper from bombardment $\mathrm{Th}+\mathrm{n}-\mathrm{II}$. He dissolved this in acid, removed the copper by precipitation of copper sulfide, removed the $\mathrm{Pa}^{233}$ by two precipitations of zirconium phosphate, and then set up the conditions for electrolysis. He succeeded in producing a good electrolytic deposition of $\mathrm{U}^{23}$ on platinum with a yield of about $93 \%$.

Isadore Perlman, Fontana, Prestwood and Bonner began the ether extraction of the 200 pounds of UNH of Batch II.

Tuesday, January 20,1942

Isadore Perlman, Fontana, Prestwood and Bonner finished the first ether extraction of Batch II.

David S. Breslow, a Ph.D. from Caltech whom I recruited with the help of my friend Saul winstein of UCLA, has started work with me in room 311, Gilman Hall. He is an organic chemist and $I$ am asking him to work on the problem of devising a szilard-Chalmers method for the concentration of the radioactive isotopes $\mathrm{U}^{237}$ and $\mathrm{U}^{239}$ formed in the neutron bombardment of uranium. I prepared the following memorandum for his use as guidance in this research program:

"I. Organic

"Salicylahdehyde-o-phenylendiamine uranyl, about $10 \mathrm{gm}$.

"Bombard with neutrons, add about $100 \mathrm{mg}$ uranyl nitrate (for carrier), distribute between organic solvent (chloroform or ?) and water. The water will contain the 7 -day $U^{237}$ (formed by $U^{238}(n, 2 n) U^{237}$ ), the $100 \mathrm{mg}$ of uranyl nitrate and all the radioactive fission products. Consult Hamaker for microchemical procedure for separation of uranyl nitrate from fission products by distribution of $\mathrm{UO}_{2}\left(\mathrm{NO}_{3}\right)_{2} \cdot 6 \mathrm{H}_{2} \mathrm{O}$ between ether and water. (This works only with saturated solutions. The uranyl nitrate goes into the ether.) Make several distributions to get pure uranyl nitrate. 
"II. Inorganic

"Dissolve about $10 \mathrm{gm}$ uranyl sulfate in about $10 \mathrm{cc} 6 \mathrm{~N} \mathrm{H}_{2} \mathrm{SO}_{4}$ and add about $10 \mathrm{cc}$ of ethyl alcohol. Add about $20 \mathrm{mg}$ uranous sulfate (for carrier material) and bombard with neutrons. (An alternate procedure might use uranyl oxalate, the oxalate replacing the ethyl alcohol as a reducing agent, or might use some other adequate reducing agent.) Uranous fluoride is precipitated by the addition of hydrofluoric acid. (This separates the uranous from the uranyl because uranyl fluoride is soluble.) The uranous fluoride contains some fission products which must be separated from it. This might be accomplished by one of the following methods. (In some cases carrier materials for the fission products should be added.)

"1. Dissolve the uranous fluoride in conc. nitric acid (which oxidizes it to the uranyl form), and prepare $\mathrm{UO}_{2}\left(\mathrm{NO}_{3}\right)_{2} \cdot 6 \mathrm{H}_{2} \mathrm{O}$ by crystallization. Then use the microchemical procedure for the separation of uranyl nitrate from the fission products by distribution between ether and water. (See Hamaker.)

"2. Dissolve the uranous fluoride in conc. nitric acid and, with lanthanum and cerium carrier present, precipitate lanthanum and cerium fluorides by the addition of hydrofluoric acid. By this operation all of the insoluble fluorides which came down with the uranous fluoride are separated from the uranyl uranium which remains in solution. This cycle could be repeated by reducing the uranium to the uranous form, precipitating uranous fluoride, dissolving this in nitric acid and precipitating lanthanum and cerium fluorides from the uranyl solution. Or an alternate method of purification of the uranium might be to precipitate and reprecipitate it as sodium uranyl acetate. (See Wahl.)

"3. Dissolve the uranous fluoride in conc. nitric acid, make the proper neutralization (see Wahl) and precipitate and reprecipitate as sodium uranyl acetate. This precipitation might be combined with any of the other procedures (as suggested above in 2).

"4. Dissolve the uranous fluoride in conc. nitric acid, evaporate and, after addition of ferric salt as carrier, precipitate ferric hydroxide by the addition of ammonium carbonate solution. (The uranium remains in solution.) Then precipitate the uranium by adding carbonate-free sodium hydroxide. This cycle can be repeated several times."

Wednesday, January 21,1942

Today Stoughton bombarded $1 \mathrm{gm}$ of thorium nitrate from which the thorium decay products had been removed by coprecipitation with lead and bismuth sulfide and barium sulfate immediately before the bombardment. The thorium nitrate was bombarded from 9:00 a.m. to 10:00 a.m. with neutrons produced by the irradiation of a beryllium target with 
150 microamperes of deuterons in the Berkeley 60-inch cyclotron (bombardment no. Th+n-III).

The neutron bombarded thorium nitrate was brought to room 311, dissolved and the thorium precipitated as the iodate with completion of precipitation at 10:15 a.m. A portion of this was mounted on cellophane and the beta radiation of the $\mathrm{Th}^{233}$ was measured and the decay followed, on step 2, with our Lauritsen electroscope no. 9, which will later be calibrated through the use of a Geiger-Mueller counter so that its readings can be translated into an absolute beta particle disintegration rate for $\mathrm{Th}^{23}$.

After the decay of the $\mathrm{Th}^{233}$ to the daughter $\mathrm{Pa}^{233}$, the intensity of the electron radiation from the latter will be measured with our calibrated Geiger-Mueller counter no. z-32. The purpose of this experiment is to measure the ratio of the conversion electrons plus nuclear beta particies of the daughter $\mathrm{Pa}^{233}$ to the beta particles of the parent $\mathrm{Th}^{233}$. The decay of the $\mathrm{Th}^{23}$ had proceeded far enough at $11: 50 \mathrm{a} . \mathrm{m}$. so the sample could be shifted to the Geiger-Mueller counter and the measurements were continued on this throughout the afternoon.

The plan is to follow this decay until the 25.6-hour $\mathrm{Th}^{2.31}$, produced in the bombardment by the reaction $\operatorname{Th}^{232}(n, 2 n) \operatorname{Th}^{23 i}$, has decayed so that the intensity of the beta radiation from $\mathrm{Pa}^{233}$ can be determined.

Approximately 20 pounds of UNH in the water layer from the first ether extraction of Batch II was evaporated to produce a form suitable for the second ether extraction. I had a conference with Isadore Perlman, Fontana, Prestwood and Bonner to discuss our program for working out the subsequent steps in the isolation of the $\mathrm{UX}_{1}$ and $\mathrm{U}^{234}$ which will entail practice runs with the procedures that we are contemplating; namely, the separation of the $\mathrm{UX}_{1}$ with ceric iodate (or thorium iodate) from the lanthanum carrier, the later separation of the undecayed UXI from the daughter $U^{234}$ with $\mathrm{CeF}_{4}$ (or $\mathrm{ThF}_{4}$ ) carrier, and isolation of the final product $\mathrm{U}^{234}$ by electrolysis.

Resuming our informal bi-weekly seminars in nuclear chemistry and nuclear physics after the break between semesters, this afternoon Dr. Edwin F. Orlemann, an instructor in the Department of Chemistry with an interest in nuclear chemistry, reported on energy production in stars; David Altman gave a literature report on "Isotopic Exchange Between Carbon Tetrabromide and Bromine" (J. H. Hodges and A. S. Miceli, J. Chem. Phys. 9, 725 [1941]).

Thursday, January 22, 1942

The crystallization of the 20 pounds of UNH from Batch II was completed and with a second ether extraction the UX ${ }_{1}$-containing fraction was reduced to about 2 pounds of UNH.

The work to outfit room 315 as a chemistry laboratory is near completion. The water, gas and vacuum lines, sink and hood have been installed 
and desks have been moved in to provide a headquarters for Isadore Per]man, Fontana and Bonner. It is intended that this will be a clean room from the standpoint of excluding radioactive contamination and that the final electrolysis procedure for the isolation of $\mathrm{U}^{234}$ will be carried out here by Perlman and Fontana. Prestwood and Auerbach are making their headquarters in room 301 where the large scale ether extractions of UNH are taking place.

Professor Lawrence received a letter from Harold C. Urey dated January 20, 1942 suggesting a process for the separation of element 94 from neutronirradiated uranium involving fluorination to produce a 94 hexafluoride which he expects to be volatile. This would separate the 94 from many of the fission products, after which the 94 hexafluoride would be separated from uranium hexafluoride by fractional distillation. He suggests that work on this might be done by Robert D. Fowler at Johns-Hopkins University where they have an excellent fluorine generator.

I wrote to David C. Grahame of the Department of Chemistry at Amherst College inquiring whether he might be interested in coming back to Berkeley to work on our project, which is one of high priority. I suggested a six months leave of absence. Dave was my partner in carrying out the research for our Ph.D.s at Berkeley.

Friday, January 23, 1942

The decay to date of the $\mathrm{Pa}^{233}$ in samples $\mathrm{Th}+\mathrm{n}-\mathrm{I}-\mathrm{Pa}-\mathrm{II}$ and -III indicates that the half-life of $\mathrm{Pa}^{233}$ is closer to 27 days than to 25 days as previously assumed.

Wahl has made range measurements in air and in aluminum on the alpha particles from $94^{239}$ sample no. U,n-6-1, and the 50-year 94 isotope sample U,D-1-1 (with the cellophane covers removed) using comparisons with the range of Po ${ }^{210}$ alphas (which is accurately known as $3.92 \mathrm{~cm}$ in air) and the conversion factor of Livingston and Bethe (Livingston and Bethe, Rev. Mod. Phys. 9, 276 [1937]) of $1.52 \mathrm{mg}$ per sq. $\mathrm{cm}$. Al per $\mathrm{cm}$ of air. He finds for $94^{239}$ a range of $3.62 \mathrm{~cm}$ in air and $3.71 \mathrm{~cm}$ of air equivalent in aluminum, and for the 50-year 94 isotope, a range of $4.09 \mathrm{~cm}$ in air and $4.13 \mathrm{~cm}$ of air equivalent in aluminum. Thus, average values of the range of the alpha particles, at standard conditions of $760 \mathrm{~mm} \mathrm{Hg}$ pressure and $15^{\circ} \mathrm{C}$. , are $3.65 \mathrm{~cm}$ for $94^{239}$ and $4.10 \mathrm{~cm}$ for the 50 -year 94 isotope. These ranges correspond to alpha energies of about $5.15 \mathrm{Mev}$ for $94^{23.9}$ and 5.5 Mev for the 50-year 94 isotope.

It is interesting to note that the alpha energy $(5.5 \mathrm{Mev})$ and halflife for the 50-year 94 place it on the Geiger-Nuttal plot for the $4 n+3$ family (the uranium-actinium family) of natural radioactive isotopes. From our three choices of mass number $(238,236$ or 235), the mass number 235 corresponds to the $4 \mathrm{n}+3$ designation and hence we might, alternatively, tentatively refer to the "50-year $94 "$ as $94^{235}$. (The values for the alpha energy, $5.15 \mathrm{Mev}$, and half-life, $3 \times 10^{4}$ years, of $94^{239}$ fit fairly well on the Geiger-Nuttal plot for the $4 \mathrm{n}+3$ family, as might be expected for an isotope with mass number 239 which fits into the main line of decay of the $U^{235}$ family.) 
I finally received today a letter from Vannevar Bush dated January 21, 1942 informing me that I have been designated, upon the recommendation of Dr. James B. Conant, Chairman of the National Defense Research Committee, as Official Investigator in connection with contract OEMsr-206 (Symbol no. 319) between the Office of Scientific Research and Development and The Regents of the University of California. He indicated that this has been assigned to section S-1, the section of which Dr. Briggs is Chairman. This is to cover the continuation of my work on $U^{23}$ and $93^{237}$ which has been underway for some months.

Monday, January 26, 1942

The third ether extraction was completed with Batch II after a good deal of trouble with sludge due to impurities, but it was possible to extract a good deal of the $\mathrm{UX}_{1}$ by acid leech. The aqueous phases were then turned over to Fontana and Bonner for extraction of the UX $\mathrm{U}_{1}$.

Professor Lawrence responded to Professor Urey's letter of January 20, 1942 indicating that he has discussed with me the problem of removing 94 from large amounts of uranium. He indicated that we have a process in which much of the uranium is separated by crystallization of UNH and further separated by partition between ether and water, after which the 94 can be isolated from the aqueous phase by methods that we have developed here. He suggested that the relative merits of this procedure and the fluorination procedure which he (Urey) has suggested might be discussed with Urey when he visits us. I helped Professor Lawrence in the preparation of this letter to Urey.

Lawrence's large project at Berkeley has been given A-1-a priority and assigned the temporary number SSRC-1.

Tuesday, January 27,1942

This morning David Breslow and John Hamaker had a bombardment of 50 gm UNH (containing a little $\mathrm{U}^{+}{ }^{4}$ ) with fast neutrons from 100 microamperehours of deuterons on beryllium at the 60-inch cyclotron (bombardment no. $\mathrm{U}, \mathrm{n}-14$ ) for the purpose of testing a possible szilard-Chalmers method for the concentration of $\mathrm{U}^{237}$. They dissolved the UNH and $\mathrm{U}^{+}{ }^{4}$, potential carrier for any $\mathrm{U}^{23} 7$ that might have been reduced to this oxidiation state during the bombardment, and precipitated $\mathrm{UF}_{4}$. They then dissolved the UF 4 in nitric acid, crystallized $\mathrm{UNH}$, extracted the uranyl uranium in ether to separate the $U^{237}$ from fission products. Measurements on this $\mathrm{U}^{237}$ fraction did not show any increased specific activity, indicating that this attempt at a szilard-Chalmers concentration method has been unsuccessful due to exchange of the liberated $\mathrm{U}^{237}$ atoms with the $\mathrm{UO}_{2}{ }^{+*}$. It is apparent that it will be necessary, in order to effect a concentration, to use an organic compound in which the $U^{237}$ liberated as a result of neutron recoil does not exchange with the undisturbed uranium in the bulk of the starting material. 
This evening wahl performed an oxidation experiment using 94 tracer in $3 \mathrm{M} . \mathrm{H}_{2} \mathrm{SO}_{4}$ with $\mathrm{PbO}_{2}$ as oxidizing agent at $80^{\circ} \mathrm{C}$. and found that 94 is not oxidized. Element 93 tracer was not used in this experiment. It is planned to conduct some experiments devoted exclusively to the oxidation properties of element 94.

He also started experiments on the oxidation of 94 by $0.1 \mathrm{M}$. $\mathrm{Cr}_{2} \mathrm{O}_{7}^{-2}$ and $0.1 \mathrm{M}$. $\mathrm{BrO}_{3}{ }^{-}$in $1 \mathrm{M} . \mathrm{H}_{2} \mathrm{SO}_{4}$ at $80^{\circ} \mathrm{C}$. with the plan to let these continue overnight.

Wednesday, January 28,1942

Stopping his 94 oxidiation experiments with $0.1 \mathrm{M} . \mathrm{Cr}_{2} \mathrm{O}_{7}^{-2}$ and $0.1 \mathrm{M}$. $\mathrm{BrO}_{3}{ }^{-}$in $1 \mathrm{M} . \mathrm{H}_{2} \mathrm{SO}_{4}$ at $80^{\circ}$ C. after 11 hours, wahl found that 94 was quantitatively oxidized in both instances.

This afternoon wahl took the water extract from Breslow's UF 4 precipitate which has been dissolved in $\mathrm{HNO}_{3}$, converted to UNH and extracted in ether yesterday and performed an oxidation-reduction cycle using $\mathrm{BrO}_{3}^{-}$as oxidizing agent, $\mathrm{SO}_{2}$ as reducing agent, and $\mathrm{LaF}_{3}$ as carrier to isolate an element 93 fraction $(U, n-14-A)$. The purpose is to see whether element 93 can be extracted from neutron bombarded UNH using an initial step with UF 4 as carrier and hence eliminating the use of rare earth carrier at this stage. He found a substantial yield of $93^{239}$ but made no quantitative measurement of the yield.

A similar oxidation-reduction cycle was used to isolate element 93 from the filtrate of Breslow's UF 4 precipitate (U,n-14-1).

Preparatory toward taking the big step of dissolving sample $T h+n-I-P a-I$ (prepared on November 18) in order to produce our large sample of $\mathrm{U}^{233}$, Gofman today carefully compared its beta radioactivity with that of Th+n-I-Pa-III which has been carefully measured with our calibrated ionization chamber FP-54 setup. In order to measure samples of such a high intensity, he used our thick-windowed ionization chamber filled with freon (window thickness $130 \mathrm{mg} \mathrm{Al} / \mathrm{cm}^{2}$ ) and filtered the radiation through $225 \mathrm{mg} \mathrm{Al} / \mathrm{cm}^{2}$. He measured a deflection of $216 \mathrm{~mm}$ compared to $29 \mathrm{~mm}$ for sample Th+n-I-Pa-III, indicating that the former has 7.5 times as much $\mathrm{Pa}^{23}$ (plus $\mathrm{U}^{233}$ ) as the latter.

Gofman then took the plunge. Under the assumption that he and Stoughton have now developed an adequate procedure for the separation of the daughter $\mathrm{U}^{23}$ from the undecayed $\mathrm{Pa}^{233}$, he scraped the material of sample $T h+n-I-P a-I$ off the copper plate, then washed the plate with $12 \mathrm{M}$. HCl until it showed less than $0.1 \%$ of the original gamma activity. He added a few drops of $\mathrm{HNO}_{3}$, then evaporated to a small volume. 


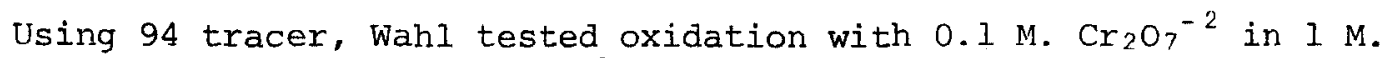
$\mathrm{H}_{2} \mathrm{SO}_{4}$ solution for 13 hours at $80^{\circ} \mathrm{C}$. and found that the 94 is completely oxidized. He also tested the oxidation of tracer 94 with $0.1 \mathrm{M}^{-\mathrm{BrO}_{3}}{ }^{-}$in $1 \mathrm{M}$. $\mathrm{H}_{2} \mathrm{SO}_{4}$ for 13 hours at $80^{\circ} \mathrm{C}$. and found that $30 \%$ of the 94 is oxidized.

Resuming his experiments to test simultaneously the oxidation of 93 and 94 , he used $0.1 \mathrm{M}$. $\mathrm{IO}_{3}{ }^{-}$in $1 \mathrm{M}$. $\mathrm{H}_{2} \mathrm{SO}_{4}$ for about 30 minutes at $80^{\circ} \mathrm{C}$. and found partial oxidiation of 93 but not of 94 . He tested $0.1 \mathrm{M} . \mathrm{BrO}_{3}^{-}$ with excess $\mathrm{Br}^{-}$in $1 \mathrm{M}$. $\mathrm{H}_{2} \mathrm{SO}_{4}$ for about 30 minutes at $80^{\circ} \mathrm{C}$. and found that neither 93 nor 94 is oxidized, made observations on $0.3 \mathrm{M}^{2} \mathrm{TI}^{+3}$ in $1 \mathrm{M}$. $\mathrm{H}_{2} \mathrm{SO}_{4}$ for 30 minutes at $80^{\circ} \mathrm{C}$. and found that neither 93 nor 94 is oxidized, tried $0.1 \mathrm{M}$. $\mathrm{H}_{5} \mathrm{IO}_{6}$ in $1 \mathrm{M}$. $\mathrm{H}_{2} \mathrm{SO}_{4}$ for one hour at $80^{\circ} \mathrm{C}$. and found that both 93 and 94 are oxidized. He finally used solid $\mathrm{PbO}_{2}$ with $5 \mathrm{M} \mathrm{H}_{2} \mathrm{SO}_{4}$ for 45 minutes at $80^{\circ} \mathrm{C}$. and found that neither 93 nor 94 is oxidized.

Proceeding with his work started yesterday, Gofman continued evaporation of his volume until $2 \mathrm{cc}$ were left, passed in $\mathrm{H}_{2} \mathrm{~S}$ to precipitate copper sulfide. The filtrate was then made $8 \mathrm{M}$. in $\mathrm{HCl}$ of total volume $10 \mathrm{cc}$, zirconium oxychloride added and the zirconium precipitated as zirconium phosphate by the addition of phosphoric acid. He then precipitated a second zirconium phosphate to further remove the $\mathrm{Pa}^{23}$, after which he reduced the volume by evaporation to $2 \mathrm{cc}$ and went home for the night.

Fontana and Bonner today completed the extraction of the UX, from UNH Batch II. They have performed two more ether extraction cycles, then diluted the final aqueous solution and precipitated the rare earths, present as impurities, as the fluoride. It was not necessary to add rare earth carriers at this stage. The rare earth fluoride was reprecipitated four times in order to completely remove impurities, including the uranium which was kept in the soluble $\mathrm{UO}_{2}{ }^{+}$state by the presence of $\mathrm{HNO}_{3}$. The final precipitate was dissolved in acid and, after the addition of $\mathrm{Ce}^{+++}$carrier, ceric iodate (together with some thorium iodate due to the thorium present in the original UNH) was precipitated from acid solution to separate the $\mathrm{UX}_{1}$ from the other rare earths. The iodate precipitate was dissolved in acid, reprecipitated, then redissolved again and the $\mathrm{Ce}^{++++}$reduced to $\mathrm{Ce}^{+++}$ by the addition of $\mathrm{H}_{2} \mathrm{O}_{2}$. Then the addition of iodate precipitated the thorium iodate (carrying the $\mathrm{Ux}_{1}$ ), leaving the $\mathrm{Ce}^{+++}$behind. This step was repeated in order to further purify the $\mathrm{UX}_{1}$. This precipitate was then dissolved and $\mathrm{ThF}_{4}$ was precipitated in the presence of $\mathrm{HNO}_{3}$ by the addition of $\mathrm{HF}$, then redissolved in acid and similarly reprecipitated several times using new chemical vessels at each step in order to make certain the removal of all of the uranium. The final precipitate was then dissolved and set aside to allow its $\mathrm{UX}_{1}$ content to decay to daughter $\mathrm{U}^{234}$.

\section{Friday, January 30,1942}

Gofman spent the entire day carefully carrying out eight successive zirconium phosphate precipitations, to continue to remove $\mathrm{Pa}^{23}$ (in continuance of his work of yesterday) from the $U^{23}$ in solution. 
Continuing his experiments to test oxidizing agents for 93 and 94 this afternoon and evening, wahl tested $0.1 \mathrm{M}$. $\mathrm{MnO}_{4}{ }^{-}$in $1 \mathrm{M} . \mathrm{H}_{2} \mathrm{SO}_{4}$ for 30 minutes at $80^{\circ} \mathrm{C} . ;$ he then cooled the solution to room temperature and held it there for three hours, made the solution $0.1 \mathrm{M}$. in $\mathrm{Mn}^{++}$with the result that the subsequent $\mathrm{LaF}_{3}$ precipitate carried the 93 (indicating that it is in the reduced state) but did not carry the 94 (indicating that it is in the oxidized state). He repeated this experiment without the subsequent addition of $\mathrm{Mn}^{++}$with the result that both 93 and 94 are in the oxidized state (that is, are not carried by the $\mathrm{LaF}_{3}$ ). Evidently $\mathrm{Mn}^{++}$can reduce 93 faster than 94 under these conditions.

He then performed a similar experiment with $0.1 \mathrm{M}$. $\mathrm{MnO}_{4}{ }^{-}$in $1 \mathrm{M}$. $\mathrm{H}_{2} \mathrm{SO}_{4}$ with the subsequent addition of $\mathrm{TI}^{+}$as a reducing agent with the result that again the 93 is reduced and the 94 is not.

He next made observations of $0.1 \mathrm{M} . \mathrm{BrO}_{3}{ }^{-}$in $1 \mathrm{M} . \mathrm{H}_{2} \mathrm{SO}_{4}$ in the presence of $10^{-4} \mathrm{M}$. $\mathrm{Ce}^{+3}$ for 30 minutes at room temperature. In this case he separated the rare earth fluoride precipitate, then added $\mathrm{SO}_{2}$ to the filtrate, made a LaF 3 precipitate, in which he found the 93 tracer (indicating that 93 is oxidized) and no 94 tracer (indicating that 94 is not oxidized). He repeated this procedure with $0.1 \mathrm{M} . \mathrm{H}_{5} \mathrm{IO}_{6}$ in $1 \mathrm{M} . \mathrm{H}_{2} \mathrm{SO}_{4}$ plus approximately $10^{-3} \mathrm{M}$. Ce $\mathrm{Ce}^{4}$ for 30 minutes at $80^{\circ} \mathrm{C}$. and found that both the 93 and 94 are oxidized.

Saturday, January 31,1942

Working all day in continuance of his isolation of $\mathrm{U}^{23}$ from sample $\mathrm{Th}+\mathrm{n}-\mathrm{I}-\mathrm{Pa}-\mathrm{I}$, Gofman removed successively eight more zirconium phosphate precipitates in his effort to eliminate undecayed $\mathrm{Pa}^{23}$ to as great an extent as possible.

This afternoon stoughton made another bombardment (no. Th $+n-I V$ ) of thorium with neutrons with the aim of measuring the $\mathrm{Th}^{233} \rightarrow \mathrm{Pa}^{233}$ decay to determine the conversion electrons of $\mathrm{Pa}^{233}$. The thorium nitrate was cleansed of natural decay products through the precipitation of lead and bismuth sulfide and lead and barium sulfate immediately before the bombardment. He bombarded one gram of thorium nitrate with neutrons produced by the irradiation of a beryllium target with 50 microamperes of $16 \mathrm{Mev}$ deuterons from 5:00 to 5:55 p.m. He has modified the chemistry in order to produce a cleaner sample of $\mathrm{Th}^{233}$. His procedure in this case was to first precipitate the dissolved thorium as the iodate, then dissolve this in concentrated $\mathrm{HCl}$ with the addition of a number of fission product carriers. From this solution zirconium phosphate was precipitated twice and from the filtrate the thorium was precipitated as the fluoride at $6: 43 \mathrm{p} . \mathrm{m}$. A portion of this was then mounted on cellophane and the radiations of $\mathrm{Th}^{23}{ }^{3}$ measured with electroscope no. 9 with the sample placed on step 2, the measurements beginning at $7: 20$ p.m.

He used another portion of the sample to determine the efficiency of Lauritsen electroscope no. 9 for the beta radiation of $\operatorname{Th}^{23}$ by making measurements with the electroscope and effecting his absolute calibration 
through the use of Geiger-Mueller counter no. z-32 (counting efficiency, 29\%). He finds a sensitivity for $\mathrm{Th}^{23} 3$ beta radiation of 2.03 divisions per second per microcurie on step 2 and, from the known relationship of step 1 to step 2, a sensitivity of 5.3 divisions per second per microcurie for step 1 .

By 8:30 p.m. the main sample had decayed sufficiently so that he could continue the measurements on Geiger-Mueller counter no. $\mathrm{z}-32$. He continued to follow the decay until a little past midnight. The plan is to continue to follow the decay on Geiger-Muellex counter no. Z-32 until all of the $\mathrm{Th}^{23}$ has decayed and the intensity of the $\mathrm{Pa}^{23}$ daughter of $\mathrm{Th}^{23} \mathrm{can}^{3}$ be established.

Sunday, February 1, 1942

Gofman took out his 17 th zirconium phosphate precipitate, then evaporated the remaining solution, containing the daughter $\mathrm{U}^{23}$, to a volume of approximately $0.15 \mathrm{cc}$. He then added $5 \mathrm{cc}$ of water, $0.02 \mathrm{cc}$ of glacial acetic acid, warmed the solution to insure that everything was dissolved, poured it into his electrolytic cell, and began electrolysis onto a platinum plate at 4:15 p.m.

Today I sat down and wrote a summary of the status of the work of my group, for use in a report to be sent by Lawrence to $J$. B. Conant, Chairman NDRC, as follows:

"A continuation of the investigation of the chemical properties of element 94 is under way. These experiments are designed to learn as much as possible about the chemistry of 94 and are using $94^{235}$, an alpha emitter of about 100 years half-life formed in the deuteron bombardment of uranium, as the tracer isotope. Particular emphasis is being placed on devising procedures to be used for the separation of $94^{239}$ from very large amounts of uranium. In addition, chemical methods are being developed for the complete separation of elements 93 and 94 from each other, using $93^{239}$ and $94^{235}$ as tracers, in order that $93^{237}$ might be isolated for the measurement of its neutron fission cross sections and spontaneous fission rate. The study of the chain beta-decay leading to $94^{239}$ is being made in order to weigh more accurately our samples of $94^{2.39}$ so as to arrive at more accurate values for its fission properties.

"Chemical procedures are being developed for the isolation of $\mathrm{U}^{23}$, which is the end product formed after a chain beta-decay when thorium is bombarded with slow neutrons. About 12 pounds of thorium nitrate have already been subjected to an intense neutron bombardment, and it is planned soon to isolate several micrograms of $\mathrm{U}^{23}$ to be used for the measurement of its neutron fission cross sections and spontaneous fission rate.

"Chemical procedures are being developed for the isolation of UX, from large amounts of uranium. The UXI decays to $]^{234}$, and it is planned, using several hundred pounds of uranyl nitrate, to isolate about a microgram of $\mathrm{U}^{234}$ in order to measure its neutron fission cross sections and spontaneous fission rate. 
"A search is being made in several ores, especially Canadian pitchblende, for elements 93 and 94 in order to see whether any isotopes of these elements exist in nature.

"Experiments are under way to develop a method for concentrating radioactive uranium, $\mathrm{U}^{237}$ or $\mathrm{U}^{239}$, by the szllard-Chalmers method. Two different methods are being tried, namely, (1) the use of organic compounds of uranium and (2) an inorganic method which takes advantage of the two valence states of uranium."

This evening Wahl tested the oxidizing power of $0.1 \mathrm{M}^{2} \mathrm{MnO}_{4}{ }^{-}$in $1 \mathrm{M} . \mathrm{H}_{2} \mathrm{SO}_{4}$ for 30 minutes at $80^{\circ} \mathrm{C}$. followed by cooling to room temperature, the addition of excess $\mathrm{Br}^{-}$and standing for 90 minutes, with the result that the 93 is oxidized and the 94 is not.

Monday, February 2, 1942

Working past midnight, Gofman terminated his electrolysis at 1:15 a.m. this morning, removed the sample and gave it the formal sample no. Th+n-IPa-I-U-I, which we decided to name more colloquially Sample J. This avoids the use of the designation Sample $I$, the next letter in the line of designation Sample I, the next letter in the line of designation of our major samples, to eliminate confusion with the Roman numeral I.

Gofman is beginning to use room 234 in the old Chemistry Building, which has been assigned to us to help us meet our expanding space requirements; this has served until this semester as a laboratory for the undergraduate course in quantitative chemical analysis (Chemistry 5). He will use this room for the measurement of the slow and fast neutron fission cross sections of $\mathrm{U}^{23}{ }^{3}$ with neutrons from our $300 \mathrm{mg}$ Ra-Be source, as well as the spontaneous fission rate of $\mathrm{U}^{233}$.

After going home for some sleep, Gofman came back this evening to make the initial test on whether $U^{23}$ undergoes fission with slow neutrons using Sample J. He used our $300 \mathrm{mg}$ Ra-Be neutron source and placed Sample $J$ on one electrode of an ionization chamber connected to a linear amplifier adjusted to discriminate against the high alpha particle emission rate in passing spontaneous fission signals to the recording system. Our Uranium Standard No. 6 (U-St-6), containing 200 micrograms of natural uranium and hence 1.4 micrograms of $\mathrm{U}^{235}$, will be used as a comparison standard. Measurements on Sample $J$ were made with and without a cadmium shield in order to determine the effect of slow neutrons.

Beginning his counting at 9:44 p.m., Gofman found 39 counts per hour which could be attributed to the slow neutron fission of $v^{233}$ in our sample J. This, then, represents the first demonstration of the slow neutron fissionability of $\mathrm{U}^{23}$, but quantitative results will await the determination of the weight of the $\mathrm{U}^{233}$ by measurements of its alpha counting rate and the use of the half-life of $U^{233}$ as determined from our $U^{233}$ alpha growth experiments from the decay of $\mathrm{Pa}^{233}$ in samples $\mathrm{Th}+\mathrm{n}-\mathrm{I}-\mathrm{Pa}-\mathrm{II}$ and -III. 
Continuing his work of yesterday, Gofman determined in the early morning hours that the slow neutron fission counting rate of U-St-6 is about 17 counts per hour. This, then, gives us a very preliminary measure of the slow neutron fission ratio

$$
\frac{\text { Sample J }}{U-S t-6}=\frac{39}{17}=2.3 \text {. }
$$

Our determination of the relative cross sections of $U^{233}$ and $U^{235}$ will await determination of the weight of $\mathrm{U}^{233}$ in sample $\mathrm{J}$.

It is clear that the use of our $300 \mathrm{mg}$ Ra-Be source makes the determination of the slow neutron fissionability of $\mathrm{U}^{233}$ a much simpler task than our determination of the slow neutron fissionability of $94^{239}$ last May using neutrons from the 37-inch cyclotron.

After going home for some sleep, Gofman returned this afternoon to continue his measurement of the comparative slow neutron fission in Sample $J$ and $U-S t-6$ (containing 1.4 microgram of $U^{235}$ ) using our $300 \mathrm{mg}$ Ra-Be source. He found a ratio for slow neutron fission of

$$
\frac{\text { Sample J }}{\text { U-St-6 }}=\frac{48}{12}=4 \text {. }
$$

Gofman then decided to make a preliminary look for spontaneous fission in Sample $J$ starting the counting at 11:08 p.m.

Using a number of $\mathrm{Pa}^{233}$ samples (from bombardment $\mathrm{Th}+\mathrm{n}$-II of last December) yesterday and today Stoughton calibrated our Lauritsen electroscopes no. 5 and no. 9 for the detection of the beta radiation from $\mathrm{Pa}^{233}$. He counted the $\mathrm{Pa}^{233}$ samples on our Geiger-Mueller counter no. Z-32 (of known counting efficiency, 29\%) and on the electroscopes. Correcting for the absorption of the radiation in the thin windows of the counter and electroscopes (using the known half thickness of $11.5 \mathrm{mg}$ per $\mathrm{sq} . \mathrm{cm}$ ), he finds for electroscope no. 5 a sensitivity of 6.25 divisions per second per microcurie when the sample is placed on step 1, 2.53 divisions per second per microcurie (step 2); and for electroscope no. 9, a sensitivity of 7.85 divisions per second per microcurie (step 1), and 3.00 divisions per second per microcurie (step 2). (The unit "microcurie" here refers to the total radiation consisting of conversion electrons as well as nuclear beta particles.)

Late this evening stoughton made an hour's bombardment of $1 \mathrm{gm}$ of thorium nitrate with neutrons at the 60-inch cyclotron (bombardment no. $T h+n-V)$, then isolated chemically a purified thorium fraction using the same procedure he used on Saturday; he will use this fraction to make another determination of the efficiency of electroscope no. 9 for $\mathrm{Th}^{23}$ beta radiation using Geiger-Mueller counter no. z-32 for absolute counting.

Wahl tested the oxidizing power of concentrated $\mathrm{HNO}_{3}$ with $2 \mathrm{M} \mathrm{H}_{2} \mathrm{SO}_{4}$ for 10 minutes at $80^{\circ} \mathrm{C}$. and found that neither 93 nor 94 is oxidized. 
Continuing his work on yesterday's bombardment (Th+n-V) early this morning, Stoughton made measurements on the $\mathrm{Th}^{233}$ beta radiation in his thorium fraction with Lauritsen electroscope no. 9 and Geiger-Mueller counter no. Z-32 and found a value for the sensitivity of electroscope no. 9 for the beta radiation of $\mathrm{Th}^{233}$ in good agreement with the value he found in his similar experiment of January 31.

Returning this morning, Gofman found that at $11: 25$ a.m. Sample J has not produced any spontaneous fission counts during the interval of 12 hours and 17 minutes since last night. He changed the "A" battery of the amplifier and then started another spontaneous fission count experiment at $8: 40$ p.m.

Gofman is expanding his work to include the investigations of the chemical properties of 93 and 94 . Today he completed a bombardment of $75 \mathrm{gm}$ of UNH with neutrons produced by the irradiation of beryllium with 200 microampere-hours of deuterons and of phosphorus with 200 microamperehours of deuterons at the 60-inch cyclotron. (This was given a bombardment number duplicative of Breslow's bombardment of January $27, \mathrm{U}, \mathrm{n}-14$. In the case of the beryllium-neutrons, his UNH had a good position next to the beryllium target, but in the case of the phosphorus-neutrons, it was not very well placed. He began the extraction of the UNH at 8:00 p.m. with ether and then performed oxidation-reduction cycles on the aqueous phase after adding carriers for the fission products. The plan is to use $\mathrm{BrO}_{3}{ }^{-}$as the oxidizing agent, $\mathrm{SO}_{2}$ as the reducing agent, and $\mathrm{LaF}_{3}$ as the carrier for the two oxidation-reduction cycles. A portion of this 93 fraction will be mounted on cellophane; he will follow the decay by placing this sample on step 2 of electroscope no. 9. The purpose of this experiment is to produce a stock of $93^{239}$ tracer and to provide Gofman with practice for this new area of investigation.

Stoughton completed his measurements on the $\mathrm{Pa}^{23}$ formed as the daughter of $\operatorname{Th}^{23}$ in the continuance of the experiment that began on January 21 (bombardment no. Th+n-III) to study the $\mathrm{Th}^{23} \rightarrow \mathrm{Pa}^{23}$ decay sequence. Using his calibration of Lauritsen electroscope no. 9, he finds the disintegration rate of the parent $\mathrm{Th}^{233}$ to be 205,000 per minute when extrapolated back to the time of its separation from daughter $\mathrm{Pa}^{233}$. The disintegration rate of the $\mathrm{Pa}^{23}$ extrapolated back to the time of the beginning of its decay (which requires a correction for the contribution of the 24.5-hour $\mathrm{Th}^{231}$ ) is 180 per minute. Using a correction factor of 1.26 for the absorption of the soft $\mathrm{Pa}^{233}$ electrons (absorption half thickness of $11.5 \mathrm{mg}$ per $\mathrm{sq}$. $\mathrm{cm}$ ) in the window $(3.9 \mathrm{mg}$ mica per $\mathrm{sq} . \mathrm{cm}$ ) of our Geiger-Mueller counter no. $\mathrm{z}-32$, we can calculate the ratio of daughter $\mathrm{Pa}^{23}$ electrons to parent $\mathrm{Th}^{23}$ electrons as

$$
\frac{180 \times 27 \times 24 \times 60 \times 1.26}{205,000 \times 25.5}=1.69
$$

using 25.5 minutes as the half-life of $\mathrm{Th}^{23}$ and 27 days as the half-life of $\mathrm{Pa}^{233}$. Analysis of the $\mathrm{Th}^{23} \rightarrow \mathrm{Th}^{231} \rightarrow \mathrm{Pa}^{233}$ decay complex indicates a half-life of 25.5 minutes for $\mathrm{Th}^{23}$. 
In our informal nuclear seminar this afternoon, which was presided over by Samuel Ruben, Robert Duffield reported on the paper "Energy and Multipole Order of Nuclear Gamma-rays" (A. C. Helmholz, Phys. Rev. 60. 415 [1941]). Two other graduate students also made reports on papers: C. N. Rice reported on "Heat Energy from Radioactive Sources in the Earth" (W. D. Urry, J. Wash. Acad. Sci. 31, 273-84 [1941]), and C. Barter reported on "Radioactive Argon $A^{37}$ " (F. K. Weimer, J. D. Kurbatov and M. L. Pool, Phys. Rev. 60, 469 [1941]).

Thursday, February 5,1942

Gofman returned and found no spontaneous fission from sample $J$ at 12:35 p.m., representing an interval of 15 hours and 55 minutes.

Gofman finished the isolation of the $93^{239}$ sample from yesterday's bombardment $(U+n-14)$ and labeled it sample no. U+n-14-1.

During the last week Bonner and Prestwood have been working on the method for the separation of the daughter $U^{234}$ from the undecayed $U_{1}$ which consists of precipitating the $\mathrm{CeF}_{4}$ (or $\mathrm{ThF}_{4}$ ) to carry the $\mathrm{UX}_{1}$, to leave the $\mathrm{U}^{234}$ behind in the $\mathrm{HNO}_{3}$ solution. Bonner has used $\mathrm{UX}_{1}$ tracer to show that the $\mathrm{CeF}_{4}$ carries the $\mathrm{Ux}_{1}$ quite completely, while Prestwood has used $U^{23}$ tracer furnished by Gofman to show that the uranium remains quantitatively in solution when the $\mathrm{CeF}_{4}$ is precipitated. After the first precipitation of $\mathrm{CeF}_{4}$ and $\mathrm{ThF}_{4}$, additional $\mathrm{Ce}^{+}{ }^{4}$ is added to the $\mathrm{HF}$ solution to make a number of additional precipitations of $\mathrm{CeF}_{4}$ in order to completely remove the Th from the $U$.

Friday, February 6,1942

Continuing his measurements on the slow neutron fission of the $\mathrm{U}^{23}$ in Sample $\mathrm{J}$ with the $300 \mathrm{mg}$ Ra-Be neutron source, Gofman found a counting rate of 40 fissions per hour with sample $J$ and 14 fissions per hour for comparison sample U-St. -6 , corresponding to a ratio between the two of 2.9 .

Wahl has made a careful measurement of the growth of alpha disintegration rates of 94 daughter sample no. U,D-1-1 (mainly 50-year 94), in which he started to measure the growth of daughter alpha radioactivity on September 10, of $94^{239}$ daughter sample no. U,n-6-1, in which he started to measure growth of daughter alpha radioactivity on September 17 , and of $94^{239}$ daughter sample no. 93-19-1, for which measurements started on July 19. He has placed the samples on one electrode of our "inside" ionization chamber no. $\mathrm{W}-3$, whose efficiency was determined to be $44 \pm 3$ percent by measuring the alpha emission rate of exactly 200 micrograms of $U$ placed in the same position. Because the alpha counting rate of sample $\mathrm{U}, \mathrm{D}-1-1$ was too high to be measured on the inside chamber, the alpha disintegration rate of a smaller sample (no. 93-14-11) containing the 50-year 94 was measured and the ratio of the alpha activity of the two samples was 
determined by using our low geometry ionization chamber-linear amplifiermagnetic field setup. (no. W-1). Thus, he has found for sample U,D-l-1 a growth of alpha disintegrations of 613 per second. From his direct measurements on sample No. U, n-6-1 he has found a rate corresponding to a growth of 258 per second, and for sample 93-19-1 he has found a total of 153 alpha particles per second. The later intensity must be corrected for the alpha particles already present when growth started on July 19; using the data of August 8 we find a correction factor of $68 / 170=0.40$, giving us the corrected value $153 \times 0.40=61$ alpha particles per second.

Saturday, February 7, 1942

As the result of a telegram from Arthur Compton, I met in Ryerson Hall at the University of Chicago yesterday and today with a group to discuss the general problem of the production of $94^{239}$ using the chain reaction in natural uranium and its subsequent separation from the uranium and fission products. Present during the meetings at various times were Arthur H. Compton, Enrico Fermi, Franklin A. Long, Norman Hilberry, Gregory Breit, John A. Wheeler, Herbert N. McCoy, Herman I. Schlesinger, Morris S. Kharasch, Herbert C. Brown, R. S. Archer, and B. L. Benbow.

In the course of the meetings I gave rather complete reports on our work at Berkeley on the identification of $94^{239}$, including the measurements of its radioactive properties and slow and fast neutron fission cross sections, our work on the radioactive properties of $93^{239} \mathrm{U}^{237}$, the 50-year 94, its parent 2.0 -day 93 , and our predictions for $93^{237}$ and $U^{233}$ which I indicated we are in the process of identifying.

I described our work on the chemical properties of 94 and our procedures for isolating 94 and 93 from large amounts of UNH and fission products using our oxidation-reduction procedure with rare earth fluoride as carrier.

As a result of this meeting I now fully realize the magnitude of the project being planned around our new element 94 and the enormity of the chemical separation problem of isolating $94^{239}$ from large amounts of uranium and almost fantastic intensities of fission products. When Compton asked me if I thought I could devise very soon a chemical process for separating 94 from such uranium and fission products-a process that could be successfully scaled up for actual use in a chemical extraction plant-I indicated that I thought I could, but I must confess to some misgivings.

One of the questions that we discussed was the matter of who might head up this chemical extraction work on 94 at the Metallurgical Laboratory here in Chicago. Compton is trying to convince Frank Long to undertake this responsibility, but Frank is doubtful that he should leave the other war work he is doing, which he regards as very important. 
We plan to extract the $93^{237}$ from the 1200 gm of UNH bombarded with the neutrons produced by the irradiation of a beryllium target with $16 \mathrm{Mev}$ deuterons in the 60-inch cyclotron for 15,000 microampere-hours last March (and which we have decided to designate bombardment no. U,n-15). The UNH was placed directly behind the beryllium target with no intervening paraffin so as to maximize the production of 6.8 -day $U^{237}$ by the reaction $U^{23}(n, 2 n)$ $\mathrm{U}^{237}$; in the intervening months, this $\mathrm{U}^{237}$ has, of course, completely decayed to its daughter $93^{237}$. Our aim is to extract this $93^{237}$, measure its alpha disintegration rate, and from this and its known yield (which determines its weight), calculate its alpha half-life. We also plan to measure the slow neutron and fast neutron fission cross section using this $93^{237}$.

In order to test the overall efficiency of his planned chemical procedure for extraction of the $93^{237}$ from bombardment no. U, n-15, wahl today started a practice run using essentially this procedure. To a $100 \mathrm{gm}$ sample of unirradiated UNH dissolved in $\mathrm{H}_{2} \mathrm{O}$ and ether to simulate his starting point, wahl added $93^{239}$ tracer (which has been isolated by Gofman from the uranium-neutron bombardment no. U, n-14 as sample no. U, n-14-1) for $93, \mathrm{U}^{233}$ as tracer for uranium, $\mathrm{Pa}^{233}$ as tracer for protactinium (both furnished by Gofman), UX ${ }_{1}$ as tracer for thorium (furnished by Fontana), and 50-year 94 (from sample no. 93-14-K) as tracer for 94 . He plans to carry through a number of oxidation-reduction cycles using $\mathrm{BrO}_{3}{ }^{-}$and $\mathrm{S}_{2} \mathrm{O}_{8}{ }^{-2}$ plus $\mathrm{Ag}^{+2}$ as oxidizing agents, $\mathrm{SO}_{2}$ as reducing agent, and rare earth fluoride as carrier. With $\mathrm{BrO}_{3}^{-}$at room temperature, the 93 but not the 94 is oxidized to the fluoride soluble state, and thus a separation of these two elements can be effected when reduced 94 is carried by rare earth fluoride (the cold bromate method for separation of 93 from 94).

Tuesday, February 10, 1942

Having worked since the day before yesterday, wahl completed his practice run for the isolation of $93^{237}$ from bombardment no. U,n-15. His measurements of the $93^{239}, \mathrm{U}^{233}, \mathrm{~Pa}^{233}, \mathrm{UX}_{1}$ and 50-year 94 tracers on the final 93 fraction show that he achieved a yield of $32 \%$ of the 93 with the removal of uranium to a level of less than 0.2 microgram, 94 to less than $0.02 \%$, protactinium less than $0.01 \%$, thorium less than $0.01 \%$ of that originally present. This clearly shows that the chemical procedure is satisfactory, although the yield of 93 can probably be improved.

Today Gofman is starting a series of electrolysis experiments for 93 using $93^{239}$ tracer (sample no. U+n-14-1) isolated last Thursday. He hopes to apply the methods that he and stoughton have developed for the electrolysis of $U$ out of acetic acid solutions to element 93 and perhaps later to element 94 . The aim is to try to find a method of separating 94-that is, $94^{239}$-from the mixture of uranium and fission products with which it will be found after the operation of a natural uranium chain reaction. 
Stoughton completed today his measurements on the intensity of electron radiation from $\mathrm{Pa}^{23}$, which is the follow-up of the experiment which began on January 31 (bombardment no. $\mathrm{Th}+\mathrm{n}-\mathrm{IV}$ ) to study the $\mathrm{Th}^{23} \rightarrow \mathrm{Pa}^{23}$ decay sequence. He finds the disintegration rate of $\mathrm{Pa}^{233}$ extrapolated back to the time of the beginning of its decay to be 227 per minute. The intensity of the parent $\mathrm{Th}^{23}$ beta radiation extrapolated back to the time of separation from daughter $\mathrm{Pa}^{233}$ is 270,000 per minute, based on the calibration of electroscope no. 9. Hence, when a correction factor of 1.26 is applied for the absorption of $\mathrm{Pa}^{233}$ beta particles in the Geiger-Mueller counter no. $\mathrm{z}-32$ window, we can calculate the ratio of daughter $\mathrm{Pa}^{233}$ electrons to parent $\operatorname{Th}^{23}$ electrons as

$$
\frac{227 \times 27 \times 24 \times 60 \times 1.26}{270,000 \times 25.5}=1.62
$$

using 25.5 minutes as the half-life of $\mathrm{Th}^{233}$ and 27 days as the half-1ife of $\mathrm{Pa}^{23}$.

I sent a letter to Professor Clifford S. Garner at the Department of Chemistry, University of Texas, an old friend who I have known since his graduate school days at the California Institute of Technology. I indicated that Professor Giauque in the Chemistry Department here is interested in having him join in one of his defense research programs; but in view of the uncertainty connected with this, I am suggesting that he might come here and work tentatively in my research group until these uncertainties are resolved. I indicated that he might start at any time it is convenient within the next month or two.

Wednesday, February 11,1942

Today Wahl started his chemical extraction of $93^{237}$ from the $1200 \mathrm{gm}$ of UNH bombarded with neutrons produced from 15,000 microampere-hours of deuterons on beryllium last March (bombardment no. U,n-15). From the yield measurements of April 3, 1941 for the 500 microampere-hour bombardment no. 93-15 of March 31, 1941, in which sample no. 93-15-1 containing $100 \mathrm{mg}$ UNH showed an intensity of $\mathrm{U}^{237}$ beta radiation corresponding to 0.26 divisions per second and sample no. 93-15-2 containing $16 \mathrm{mg}$ UNH showed 0.069 divisions per second on electroscope no. 5 (step 1), and using our calibrations of electroscope no. 5 determined on January 11,1912 as 0.94 and 1.64 divisions per second per microcurie of $\mathrm{U}^{237}$, respectively, we can calculate the yield of $\mathrm{U}^{237}$ as

and

$$
\frac{(0.26)\left(10^{3}\right)}{(0.94)(100)(500)}=0.0055 \text { microcuries per gm UNH per microampere-hour, }
$$

$$
\frac{(0.069)\left(10^{3}\right)}{(1.64)(16)(500)}=0.0053 \text { microcuries per gm UNH per microampere-hour. }
$$

Using the average value 0.0054 , this indicates that we had initially in the $1200 \mathrm{gm}$ of neutron-bombarded UNH 


$$
\frac{(0.0054)(15,000)(1200)}{10^{3}}=97 \text { millicuries of } \mathrm{U}^{237}
$$

which is equivalent to 1.2 micrograms of $U^{237}$ as calculated with the use of its 6.8-day half-life. Upon decay this has yielded 1.2 micrograms of $93^{237}$ which should be present in the $1200 \mathrm{gm}$ of UNH of bombardment no. U, n-15. (Incidentally, this yield of $\mathrm{U}^{237}$ can be compared with the yield, 0.0045 microcuries per gram of uranium element per microampere-hour, corresponding to 0.0025 micrograms per gram of UNH per microampere-hour, from the bombardment of $1.2 \mathrm{~kg}$ of UNH on February 28 to March 3, 1941 reported to Dr. Lyman J. Briggs on June 17, 1941; the smaller earlier yield, 0.0025 compared to 0.0054 , resulted from our deliberate attempt then to maximize the slow neutron flux to produce $93^{239}$, which decreases the efficiency of production of $\left.\mathrm{U}^{237}.\right)$

Wahl dissolved the $1200 \mathrm{gm}$ of UNH in ether, added $93^{239}$ tracer (furnished by Gofman from his sample U,n-14-1, Stock 1 solution of bombardment $\mathrm{U}, \mathrm{n}-14)$, separated the water layer, added rare earth carriers and other carriers for fission products, and precipitated rare earth fluoride as the initial step in the isolation procedure. This rare earth fluoride should include the sought-after $93^{237}, 93^{239}$ tracer, $94^{239}$, as well as rare earth fission products. The plan is to separate the 93 fraction very completely from 94, uranium, protactinium, thorium and fission products using a procedure similar to, but more thorough, than that used in the practice run completed yesterday. Again the procedure will consist of oxidation-reduction cycles using $\mathrm{BrO}_{3}^{-}$and $\mathrm{S}_{2} \mathrm{O}_{8}{ }^{-2}$ plus $\mathrm{Ag}^{+}$as oxidizing agents, $\mathrm{SO}_{2}$ as reducing agent, and rare earth fluoride as carrier. The procedure contemplates three separations from 94, six separations from uranium, five separations from protactinium, and five separations from thorium.

From continued measurements on the slow neutron fission of the $U^{23}$ in sample $\mathrm{J}$ with the $300 \mathrm{mg}$ Ra-Be neutron source, Gofman has found a counting rate of 45 fissions per hour with sample $J$ and 14 fissions per hour for comparison sample U-St-6, corresponding to a ratio between the two of 3.2 .

Embarking on his electrolysis experiments, Gofman is planning to investigate the electrodeposition of 93 from acetic acid solution in the reduced state and in the oxidized (with $\mathrm{BrO}_{3}{ }^{-}$) state to establish whether there is a difference that can be exploited. The indications are that the 93 is electrolytically deposited in much higher yield from oxidized than from reduced solution.

\section{Friday, February 13,1942}

During the last week Isadore PerIman and Prestwood have been developing a procedure for the recovery of $\mathrm{Ux}_{1}$ from residues which we received from the Shattuck Chemical Company in Denver; these arise from their process of converting sodium uranate to $\mathrm{UNH}$ and should include relatively high concentrations of $\mathrm{Ux}_{1}$. 
Gofman is continuing his electrolysis of 93 using some of his stock $93^{239}$ tracer. He finds that the presence of sulfuric acid reduces the electrolytic yield of 93 from oxidized solution if the concentration is too high.

Today we received 224 pounds of UNH bearing the label of the Eldorado Gold Mines Ltd., which we purchased from the Los Angeles branch of the du Pont Company (Batch III).

Sunday, February 15,1942

Today Gofman is starting his electrolysis experiments with 94 using as tracer some of Wahl's stock solution of 94 (93-14-K). He plans during the coming weeks to attempt to develop optimum conditions for the separation of 94 from the fission products. He will investigate in particular the possibility of electrodeposition of 94 from solutions in which it is present in the oxidized state to see how this differs from its electrolytic deposition from solutions in which it is present in the reduced state.

English has made a bombardment of a uranium-tellurium target with the $16 \mathrm{Mev}$ deuterons in the 60-inch cyclotron (bombardment no. U,D-2) which ended today (total of 2000 microampere-hours, estimated 1000 microamperehours on uranium). The purpose of the bombardment is to produce a sample of 50-year 94 for use as tracer and, more particularly, for use in the measurement of its spontaneous fission half-life.

Monday, February 16, 1942

This evening Stoughton bombarded $1.6 \mathrm{gm}$ of thorium nitrate, from which natural decay products had been previously removed, with neutrons from the 60-inch cyclotron from 8:15 p.m. to 10:00 p.m. (bombardment no. Th+n-VI). He immediately isolated a pure thorium fraction using a procedure essentially like that of January 31 , and began measurements of the $\mathrm{Th}^{23} 3$ decay using electroscope no. 9 at 10:29 p.m. He changed over to Geiger-Mueller counter no. z-32 at $11: 25$ p.m.

Isadore Perlman, Fontana, Prestwood, Bonner and Auerbach started the ether extraction of UNH Batch III. They started yesterday grinding the material to fine form in order to make it suitable for this process.

I wrote to H. T. Wensel, Technical Aide, Section S-l in Washington, enclosing as he had requested a report on our work for the search for elements 93 and 94 in nature which was supported by contract NDCrc-197. The report was co-authored by Arthur Wahl and me based on Wahl's work of last october. I indicated that we have used the oxidizing and reducing procedure with rare earth fluoride carrier to isolate elements 93 and 94 from $50 \mathrm{gm}$ of pitchblende from the Great Bear Lake region of Canada and found that there is no detectable amount of either alpha particle or beta 
particle emitting 93 or 94 . Morris Perlman does not yet have results from his more exhaustive search for 93 and 94 in this pitchblende.

Tuesday, February 17, 1942

Wahl has calibrated more carefully our ionization chamber FP-54 electrometer setup for the beta radiation of $93^{239}$ and, in order to make an estimate for the 2.0-day 93, he also made a calibration for the beta radiation of a $\mathrm{UX}_{1}-\mathrm{UX}_{2}$ source (consisting of $\mathrm{UX}_{2}$ beta particles with the soft $U_{1}$ beta particles removed by absorption). Various aliquote of $93^{239}$ solutions were evaporated on thin metal foils (approximately $2 \mathrm{mg}$ per sq. $\mathrm{cm}$ thickness) and measured source side down (to eliminate backscattering) on the FP-54 ionization chamber and our calibrated Geiger-Mueller counter; corrections for absorption in the thin foils and mica window of the counter were made. (The measurement of July 19, 1941 indicates a correction factor of 0.62 for backscattering from platinum.) These measurements have determined that the sensitivity of our FP-54 ionization chamber for $93^{239}$ electron radiation (with no backscattering) corresponds to a galvanometer deflection of $1.34 \times 10^{4} \mathrm{~mm}$ per microcurie (conversion electrons plus nuclear beta particles), with the sample on step 2, at our "standard conditions" of resistance and shunt setting R2S1. For UX 2 beta radiation, from similar measurements, wahl finds $6.38 \times 10^{3} \mathrm{~mm}$ deflection per microcurie for the resistance and shunt setting R2S1, from which we can interpolate that the value for the beta radiation of the 2.0 -day 93 should be $9.25 \times 10^{3}$ $\mathrm{mm}$ deflection.

It is possible now to make more accurate calculations of the half-life of $94^{239}$ from the data obtained with samples $U, n-6-1$ and $93-19-1$ on the basis of the more careful measurements of the intensity of the daughter $94^{239}$ alpha particles that resulted from the decay of the known amounts of 2.3-day $93^{239}$ and the use of the value 2.0 for the correction factor for the conversion electrons of $93^{239}$ (determined in December). For sample $\mathrm{U}, \mathrm{n}-6-1$, using the measurement of the initial intensity of the beta radiation from the $93^{239}$ isolated on september 16, 1941 (i.e., the galvanometer deflection of $1.26 \times 10^{9} \mathrm{~mm}$ at resistance and shunt setting R2S1), the calibration $1.34 \times 10^{4} \mathrm{~mm}$ deflection per microcurie of $93^{239}$, the correction factors for backscattering from platinum (0.62) and conversion electrons $(1 / 2.0)$, and the final growth of daughter $94^{239}$ alpha particles (258 per second, described on February 6), we find as our value for the half-life of $94^{239}$ using the relationship

$$
\frac{\text { (Dis. rate of parent } 93^{239} \text { ) (Half-life of } 93^{239} \text { ) }}{\text { Dis. rate of daughter } 94^{239}}=\text { half-life of } 94^{239}
$$

the value,

$$
\left[\frac{\left(1.26 \times 10^{9}\right)\left(3.7 \times 10^{4}\right)(0.62)}{\left(1.34 \times 10^{4}\right)(2.0)}\right]\left(\frac{1}{258}\right)\left(\frac{2.33}{365}\right)=2.7 \times 10^{4} \text { years. }
$$

The use of the correction factor for conversion electrons $(1 / 2.0)$ and the better value for the alpha intensity of the $94^{239}$ (258 instead of 216 
per second), accounts for the difference of this value and the rough value of $6 \times 10^{4}$ years calculated on October 13, 1941 .

Similarly, for sample 93-19-1, correcting the calculation of August 29, 1941 by incorporating the correction factor 2.0 and using the best value of the growth of alpha intensity $(61$ per second, described on February 6 ), we find

$$
\left[\frac{\left(2.4 \times 10^{8}\right)\left(3.7 \times 10^{4}\right)(0.62)}{\left(1.34 \times 10^{4}\right)(2.0)}\right]\left(\frac{1}{61}\right)\left(\frac{2.33}{365}\right)=2.1 \times 10^{4} \text { years. }
$$

Again, the use of the correction factor $(1 / 2.0)$, and the better value for the alpha intensity, accounts for the difference of this value and the rough value of $5 \times 10^{4}$ years calculated on August 29, 1941.

The calibration of our ionization chamber FP-54, which makes it possible to estimate its efficiency specifically for beta radiation of energy like that of the 2.0-day 93, and the better determination of the intensity of the daughter alpha particles, now makes it possible also to calculate a better value for the half-life of our deuteron-produced, alpha-emitting "50-year 94" using our data from sample U,D-1-1 for the 2.0-day $93 \rightarrow$ 50-year 94 decay sequence. Using the measurement of the initial intensity of the beta radiation from the $93\left(93^{239}\right.$ plus 2.0-day 93) in sample U,D-1-1 of September 10 (i.e., $4.13 \times 10^{7} \mathrm{~mm}$ of galvanometer deflection at resistance and shunt setting R2Sl), the calibration of $9.25 \times 10^{3} \mathrm{~mm}$ deflection per microcurie of 2.0-day 93, the estimate that the 2.0-day 93 accounts for $5 \%$ of the measured radiation, the correction factor for backscattering (0.62), and the final growth of daughter 50-year 94 alpha particles (613 per second, described on February 6$)$, we have for the half-life of the "50-year 94"

$$
\left[\frac{\left(4.13 \times 10^{7}\right)\left(3.7 \times 10^{4}\right)(0.05)(0.62)}{\left(9.25 \times 10^{3}\right)}\right]\left(\frac{1}{616}\right)\left(\frac{2.0}{365}\right)=46 \text { years. }
$$

By far the largest error is the estimate of the proportion (5\%) of the electrons in the initial mixture of $93^{239}$ and 2.0 -day 93 (both produced in the deuteron bombardment of uranium) corresponding to the beta particles of the 2.0-day 93. Our estimate of 5\% comes from the aluminum absorption measurements (made on september 11, 1941) on the mixture of 93 isotopes. A rough resolution of the decay curve for the mixture of 2.3 -day $93^{239}$ and 2.0-day 93 into its two components gives about the same result. A realistic estimate of the error in this is $60 \%$. We will continue to refer to our first alpha-active 94 (the isotope whose discovery represented the discovery of element 94) as the 50-year 94 with the realization that its half-life may be in error by as much as $60 \%$.

I wrote to Arthur Compton indicating that I enjoyed the opportunity of joining in the discussions at Chicago the week before last. I expressed satisfaction at how well the project is getting underway. I enclosed three copies of isotope charts summarizing the induced radioactivities in the uranium region, indicating that one copy is for him and the others for Professors Breit and wheeler. I also indicated that I haven't been able to 
think of another person whom I might recommend to head up the work on the chemical extraction of 94 should Frank Long decide not to undertake this task.

Wednesday, February 18,1942

Intermittently since his first measurements on the slow neutron fission rate of $\mathrm{U}^{23}$ started on February 2, Gofman has continued his measurements. He finds that the ratio of induced fissions, using the slow neutrons from the $300 \mathrm{mg}$ Ra-Be neutron source, with the neutron source and the ionization chamber surrounded with paraffin, on the basis of measurements made without and with the cadmium shield, corresponds to a rate for sample $J$ about three times that of the standard uranium sample (U-St-6).

Joe Hamilton received a letter from Alexander Pregel, Vice President, Canadian Radium and Uranium Corporation, New York, dated February 16, 1942 indicating pleasure that the $300 \mathrm{mg}$ Ra-Be source has arrived in good condition and suggesting that we send him the dimensions that we would like for the $1 \mathrm{gm} \mathrm{Ra-Be}$ source that we are renting from his company.

In our informal nuclear seminar this afternoon Joe Kennedy reported on the paper "New Induction Accelerator Generating $20 \mathrm{Mev}$ " (D. W. Kerst, Phys. Rev. 61, 93 [1942]), and Fred Leitz gave a talk on nuclear masses.

Thursday, February 19, 1942

Wahl completed his isolation of the 93 fraction started Wednesday of last week (sample no. 93-15-3) which he labeled Sample $\mathrm{K}$.

Measurement of the intensity of the beta particles from the $93^{239}$ tracer indicate that his yield of 93 (that is, of isotope $93^{237}$ ) through the complete separation process is 50\%. Sample $\mathrm{K}$ also shows the emission of alpha particles which, in view of the care with which all other potential alpha emitters have been eliminated in the chemical separation procedure, must be due to the new isotope $93^{237}$. The alpha counting rate, using our "inside chamber" no. $\mathrm{W}-3$, which has a calibrated efficienty of $44 \%$, is 300 counts per minute (approximately 600 disintegrations per minute). Since we know that sample $K$ contains 0.6 microgram of $93^{23} 7$ (0.5 1.2 microgram), we can calculate the alpha half-life of $93^{237}$ as about $3 \times 10^{6}$ years.

This afternoon wahl also completed the isolation of the 94 fraction $(U, n-15-4)$ from bombardment no. U,n-15 which we are labeling as sample $L$. He intends to determine its $94^{239}$ content by comparing its alpha particle disintegration rate with that of samples $F$ and 93-19-1. 
Yesterday and today Gofman has made a preliminary measurement of the range of the alpha particles of $U^{233}$ (Sample $J$ ) using our low geometry ionization chamber-linear amplifier setup no. W-2. Making comparative measurements of the absorption in aluminum foil of $\mathrm{U}^{233}$ and $\mathrm{PO}^{210}$ (sample Po-St-6) alpha particles, he finds the range of $\mathrm{PO}^{210}$ alphas to be Ionger than those of $\mathrm{U}^{233}$ by $1.34 \mathrm{mg}$ per sq. cm of $\mathrm{Al}$. Converting to range in air (conversion factor of 1.52) this difference corresponds to $0.88 \mathrm{~cm}$ of air, indicating the range of $\mathrm{U}^{23}$ is 3.92 (the air range for Po ${ }^{2 i 0}$ ) minus 0.88 or $3.04 \mathrm{~cm}$ of air at standard conditions $\left(760 \mathrm{~mm}\right.$ and $\left.15^{\circ} \mathrm{c.}\right)$. This should be considered to be a preliminary, rather rough, value.

Today Gofman is beginning his longer series of measurements to determine the spontaneous fission rate of $\mathrm{U}^{23}$ using Sample $\mathrm{J}$ with his apparatus set up in room 234 in old Chemistry Building.

The first ether extraction of Batch III was finished after much trouble with crud and impurities. This is the most impure batch that we have had so far.

Friday, February 20, 1942

The second ether extraction of Batch III was started with much attention devoted to trying to recover the $\mathrm{UX}_{1}$ associated with the sludges that have developed.

Saturday, February 21, 1942

Today Gofman measured the effective neutron intensity of our Po-Be source by placing a natural uranium sample containing 200 micrograms of uranium (U-St-10) on one electrode of our ionization chamber and then measuring the induced fission rate by neutrons slowed by paraffin with first the Po-Be neutron source and then the $300 \mathrm{mg}$ Ra-Be neutron source. He found for the former 15 fission counts in 35 minutes, corresponding to 0.43 fissions per minute, and for the latter 132 fissions in 17 minutes, corresponding to 7.76 fission counts per minute. Hence, the ratio of the effective neutron intensity of the Po-Be source to that of the $300 \mathrm{mg}$ Ra-Be source is

$$
\frac{0.43}{7.76}=0.055
$$

The second ether extraction of Batch III was concluded and an assay showed the presence of about 7,000 microcuries of $\mathrm{UX}_{1}$, indicating that the yield is about $50 \%$; this corresponds to a total of about 0.3 microgram of $\mathrm{UX}_{1}$ at this stage. This aqueous phase (volume $2700 \mathrm{cc}$ ) was turned over to Isadore Perlman and Fontana for further processing. 
Stoughton bombarded a 1 gm sample of thorium nitrate, previously purified from its natural disintegration products, with neutrons at the 60-inch cyclotron from 11:00 a.m. to 12 noon (bombardment no. Th+n-VII). He then isolated a purified thorium fraction by a procedure essentially the same as that used on January 31 and began measurements on the decay of $\mathrm{Th}^{233}$ using electroscope no. 9 at 12:35 p.m. He divided the sample into four portions, which he labeled samples no. $1,2,3$ and 4 . He then followed the decay of all four samples on electroscope no. 9. He shifted samples no. 3 and 4 to Geiger-Mueller counter no. z-32 at $2: 23$ p.m. The plan is to follow the decay of these two samples until the intensity of the the electron radiation from daughter $\mathrm{Pa}^{233}$ can be established in each case. He also intends to measure the absorption in Al of the low energy part of the $\mathrm{Pa}^{233}$ beta spectrum in order to obtain better information to correct for the absorption of the $\mathrm{Pa}^{23}$ beta particles in the GeigerMueller counter's mica window (approximately $3 \mathrm{mg}$ per sq. $\mathrm{cm}$ mica thickness). The purpose is to improve the calibration of our Lauritsen electroscope no. 9 (and hence our ionization chamber FP-54 setup) for absolute measurement of the intensities of $\mathrm{Pa}^{233}$ beta radiation, information that is needed in connection with our experiment to determine the half-life of $\mathrm{U}^{233}$.

During the last several days Perlman, Bonner and Prestwood have been practicing the separation of small amounts of Th from $\mathrm{Ce}$, a step that is used in the latter part of our procedure for the separation of $\mathrm{Ux}_{1}$. Using a mixture of $10 \mathrm{mg}$ of $\mathrm{Th}$ and $100 \mathrm{mg} \mathrm{Ce}$, they add $\mathrm{H}_{2} \mathrm{O}_{2}$ to the solution to reduce the $\mathrm{Ce}$ to $\mathrm{Ce}^{+++}$, then, after destroying the $\mathrm{H}_{2} \mathrm{O}_{2}$ by heating, precipitate the $\mathrm{Th}^{+++}$as the iodate from nitric acid solution by the addition of $\mathrm{IO}_{3}{ }^{-}$; in this procedure the $\mathrm{Ce}^{+++}$remains in solution.

\section{Tuesday, February 24, 1942}

Stoughton completed his measurements on the decay of daughter $\mathrm{Pa}^{233}$ produced in the decay of parent $\mathrm{Th}^{233}$ isolated from bombardment no. $\mathrm{Th}+\mathrm{n}-\mathrm{VI}$ on February 16, 1942. Using his absolute calibrations of electroscope no. 9 for $\mathrm{Th}^{233}$ beta radiation and Geiger-Mueller counter $\mathrm{z}-32$ results for daughter $\mathrm{Pa}^{233}$ beta radiation, and correcting for absorption in the GeigerMueller counter window, he finds a value of 1.65 for the ratio of $\mathrm{Pa}^{23}$ electrons to $\mathrm{Th}^{233}$ electrons.

Wednesday, February 25,1942

Gofman's attempts to measure the spontaneous fission rates of $\mathrm{U}^{233}$ commenced on February 19 with the apparatus set up in room 234, old chemistry Building, has been plagued by spurious counts due to electrical interference. He is therefore moving his ionization chamber, amplifier and recorder for the spontaneous fission work to his home (apartment number 106, 1890 Arch Street, Berkeley). 
I wrote to Dr. M. Pochon, Port Hope Refinery, Eldorado Gold Mines Ltd., Port Hope, Ontario, Canada, indicating that today our purchasing department has sent to his firm's New York office an order for 200 pounds of UNH. I asked that the material come from older stock in which the UNH was crystallized at least two months ago and asked him to inform me of the date of this crystallization. I have in mind receiving material in which the 24day $\mathrm{UX}_{l}$ has grown in nearly to equilibrium. I also inquired about the availability of mother liquor from which UNH has been crystallized, having in mind that in this manner we might find material with higher concentrations of $\mathrm{UX}_{1}$.

Thursday, February 26, 1942

Lawrence received a letter dated February 23, 1942 from Arthur Compton inquiring whether we might prepare some $94^{235}$ (50-year 94) for Dr. Frank Spedding. He indicated that spedding has been offered and has accepted the position of heading up the chemical program at the Metallurgical Laboratory, including the work on the extraction of 94 . He would like to have spedding start a research program on the chemistry of 94 . He noted that such 94 tracer will be required because it is essential to obtain a survey of the chemical extraction problem in the power plant at the earliest possible moment.

Professor Harold C. Urey is visiting at Berkeley today. We had a discussion concerning some distribution experiments of Professor Kraus, involving uranium and methyl ethyl ketone, with the view of perhaps performing similar experiments here with 94 and 93.

Friday, February 27, 1942

Breslow has during the last month developed his method for synthesizing what we hope will be a suitable organic uranium compound, namely the salicylaldehyde-o-phenylenediamine uranyl complex. He has found that it can readily be prepared in two steps. First the salicylaldehyde is condensed with o-phenylenediamine in hot ethyl alcohol and then the resulting complex is condensed with uranyl acetate in the same solvent. The resulting compound is then recrystallized from pyridine.

Isadore Perlman and Fontana finished the recovery of the UX, fraction from Batch III. Beginning with the aqueous phase of the second ether extraction, they added $1 \mathrm{gm} \mathrm{La}^{+++}, 0.1 \mathrm{gm} \mathrm{Ce^{+++ }}$ and diluted the solution to 12 liters, made it $0.5 \mathrm{M}$. in hydrogen ion and precipitated the rare earth fluorides by the addition of HF. These were then reprecipitated several times. The $\mathrm{Ce}^{+4}$ and $\mathrm{Th}$ (which was present in the UNH) were separated-from the $\mathrm{La}^{+++}$by the precipitation of the iodates from acid solution, then further concentration of the $\mathrm{UX}_{1}$ was achieved by reducing the $\mathrm{Ce}^{+++t^{+}} \mathrm{CO}^{+++}$ with the addition of $\mathrm{H}_{2} \mathrm{O}_{2}$ and precipitating thorium iodate. This precipitate was dissolved, $\mathrm{ThF}_{4}$ was precipitated and reprecipitated several times, using 
new glassware each time. The last $\mathrm{ThF}_{4}$ was dissolved and set aside to await the decay of its contained $\mathrm{UX}_{1}$ to daughter $\mathrm{U}^{234}$.

I have asked Isadore Perlman to expand his activities and to become familiar with the chemistry of element 94 in order to further expand our work on the separation of 94 from uranium and fission products.

\section{Saturday, February 28, 1942}

Gofman's measurements on the decay of a portion of $93^{239}$ sample U+n-14-1 isolated on February 5 has shown a half-life of 2.3 days, indicating that the sample is pure and that Gofman has mastered the chemistry of 93 quite well.

Lawrence replied to Compton's letter of February 23, 1942 indicating that we would be glad to prepare $94^{235}$ for spedding. He added, however, that Professor Urey has just been here and has been going into the chemistry of 94 very extensively with our group and has concluded that the chemistry is pretty well worked out. He also indicated that if spedding should undertake such an additional program, he should work in close collaboration with us here and begin by paying us a visit in Berkeley.

Joe Hamilton is in Denver today visiting the $S$. W. Shattuck Chemical Company ( 1805 South Bannock Street) where he is helping them collect a residue from the process they use to produce UNH in which $\mathrm{UX}_{1}$ may be concentrated. He refers to this as "Brown Mud" and hopes in the next couple of days to collect such concentrated residue corresponding to at least 600 pounds of UNH and then arrange to have it shipped to us.

We have also ordered 200 pounds of recrystallized UNH from the $\mathrm{s}$. W. Shattuck Chemical Company.

Sunday, March 1, 1942

English started to perform the chemical isolation of the 50-year 94 produced in bombardment no. U,D-2 which ended on February 15. Since this is going to be used for spontaneous fission measurements, it is necessary to make a very careful and complete chemical separation from all heavy isotopes.

Monday, March 2, 1942

Stoughton bombarded $1 \mathrm{gm}$ of thorium nitrate, previously purified of its natural decay products, with the neutrons from the 60-inch cyclotron from 7:10 p.m. to $8: 15 \mathrm{p} . \mathrm{m}$. (bombardment no. Th+n-VIII). After the bombardment he purified the thorium fraction by the usual procedure, finishing his separation procedure at $8: 53 \mathrm{p} . \mathrm{m}$. The purpose of this bombardment 
is to determine the shape of the absorption curve and the energy of the $\mathrm{Th}^{233}$ beta particles by absorption with aluminum using electroscope no. 9 with the sample on the second step. He completed the absorption measurements by 10:23 p.m. with the result that he found an end point of about $700 \mathrm{mg} / \mathrm{cm}^{2}$, corresponding to an upper energy limit of about $1.6 \mathrm{Mev}$. The initial part of the absorption curve shows a half thickness of $60 \mathrm{mg}$ per $\mathrm{sq}$. $\mathrm{cm}$ of $\mathrm{Al}$. The ratio of the ionization due to the beta particles to that due to the gamma rays is approximately 1,000, indicating that there are essentially no nuclear gamma rays in the $\mathrm{Th}^{23}$ radiation; the small amount of apparent electromagnetic radiation is probably to be attributed to bremsstrahlung. This apparently lends weight to the assumption that there are no conversion electrons present in the radiation of $\mathrm{Th}^{233}$. The shape of the absorption curve also indicates the absence of conversion electrons.

English completed today the isolation of the 50-year 94 from bombardment U,D-2. The uranium target was dissolved in a mixture of sulfuric and nitric acid, and, after the addition of lanthanum and cerium nitrate for carrier material, rare earth fluoride was precipitated by the addition of hydrofluoric acid to the solution. This rare earth fluoride contained the 94, 93 and thorium, the uranium and the protactinium (because zirconium was present) remaining in solution. The rare earth fluoride was dissolved in sulfuric acid, and from a cold potassium bromate $\left(\mathrm{KBrO}_{3}\right)$ solution the rare earth fluoride was again precipitated. In this step the 94 , in its reduced form, and the thorium and the rare earth fission products were precipitated as insoluble fluorides, and the 93 remained in solution in its oxidized form. This precipitate was dissolved in sulfuric acid, and, after the addition of potassium peroxydisulfate $\left(\mathrm{K}_{2} \mathrm{~S}_{2} \mathrm{O}_{8}\right)$ and silver ion, the rare earth fluoride was again precipitated by the addition of hydrofluoric acid. In this step the fission products were precipitated as insoluble fluorides. The 94 in the solution was then reduced by boiling the solution until the fumes of sulfur trioxide appeared and after adding sulfur dioxide, and a small amount of rare earth carrier material, the 94 was precipitated with rare earth fluoride. Of course it was necessary to perform a number of cycles in order to completely remove 93, uranium, protactinium and thorium, and in his procedure there were 2 separations from 93, 7 separations from uranium, 2 separations from protactinium, and 2 separations from thorium. The final rare earth fluoride precipitate, containing the 94 , was centrifuged directly onto a platinum disk and has a thickness of approximately $0.3 \mathrm{mg} / \mathrm{cm}^{2}$.

Professor Lawrence told me today that Compton has wired him to say that spedding might come out here to pay us a visit sometime during the month of March.

I sent a letter to Frank Spedding at the University of Chicago indicating that we are looking forward to seeing him if and when he pays us a visit sometime this month. I indicated that during his visit he will want to meet the men who have been working with me on the chemistry of 94 . I identified these as Arthur Wahl (reminding him that wahl did his undergraduate work at Iowa State, Spedding's university), John Gofman, Spofford English and Isadore Perlman. I also indicated that he will want to meet 
Kennedy and Segrè who, although they are not working on the chemistry of 94, have been working on another interesting chemical phase of the general problem, namely the preparation and measurement of samples of enriched $\mathrm{U}^{235}$ prepared by the use of the mass spectrograph.

Today we received two additional Lauritsen electroscopes purchased from the Fred $C$. Henson Company-our numbers 10 and 11 .

English finds the total yield of 50-year 94 from bombardment no. U,D-2 to be about 0.025 micrograms corresponding to $2.5 \times 10^{-5}$ micrograms per microampere-hour of deuterons. He prepared from this a sample for use to measure the spontaneous fission rate of the 50-year 94.

Wednesday, March 4, 1942

English measured the alpha intensity of his 50-year 94 sample using our calibrated, low geometry ionization chamber-linear amplifier outfit (W-2) with absorbing screens and found that it contains about 0.005 microgram of 50-year 94. Also present in this sample is about 0.05 microgram of 30,000-year $94^{239}$, which is also formed in the deuteron bombarded uranium at a 10 times higher yield, but the spontaneous fission rate of this isotope is much too low to interfere with the determination of the spontaneous fission rate of the 50-year 94 . He began immediately to make measurements on its possible spontaneous fission rate by placing the sample on one electrode of an ionization chamber connected to a linear amplifier and recording system, adjusted so as to count the impulses due to fissions while not recording any spurious large pulses that might result from fluctuations in the ionization due to the high alpha activity.

In our informal nuclear seminar this afternoon David Altman made a report on recent advances in the electron microscope. Paul Nahinsky reported on the paper "Interaction of Slow Neutrons with Nuclei" (H. Carroll, Phys. Rev. 60, 702 [1941]). Gerhart Friedlander reported on the Fermi theory of beta decay and on nuclear isomerism.

Thursday, March 5, 1942

Today Stoughton completed another calibration of our ionization chamber FP-54 electrometer setup for the beta radiation of $\mathrm{Pa}^{233}$. Using a sample of $\mathrm{Pa}^{233}$ isolated from bombardment $\mathrm{Th}+\mathrm{n}$-II on December 23, he made measurements using our calibrated electroscope no. 9 and the FP-54 ionization chamber (step 2). He finds a sensitivity of $0.058 \mathrm{~mm}$ of galvanometer deflection per microcurie of $\mathrm{Pa}^{233}$ total electron radiation (conversion electrons plus nuclear beta particles) at our standard resistance and shunt setting R5S5. This is in good agreement with the value of $0.061 \mathrm{~mm}$ which he found on January 9. 
During the last couple of days Prestwood and Bonner have investigated my idea of concentrating the $\mathrm{UX}_{1}$ in mother liquor in a procedure where most of the UNH is crystallized from solution. They find that in such a procedure about $50 \%$ of the $\mathrm{UX}_{1}$ remains behind with about $5 \%$ of the original UNH; i.e., after $95 \%$ of the UNH has been removed by crystallization. This procedure may have application as an initial step for the removal of the bulk of the uranium after neutron bombardment, hence finding application to a recovery process for element 94 since 94 will also undoubtedly concentrate in the solution in the same manner as $\mathrm{UX}_{1}$. I am asking Isadore Perlman to begin investigation of this method of concentration of 94 from neutronbombarded uranium.

Saturday, March 7, 1942

Due to the overloading of the bombardment schedule at the 60-inch cyclotron, we are hoping to have the additional use of the 45-inch cyclotron at Washington University in St. Louis. Today Martin Kamen is at Washington University and has bombarded for us this morning from 10:45 a.m. to $11: 19 \mathrm{a} . \mathrm{m}$. a $19 \mathrm{gm} \mathrm{UNH}$ sample, in a pillbox, with the fast neutrons produced by the irradiation of a beryllium target with 200 microamperehours of deuteron beam (bombardment no. U, n-16).

Monday, March 9, 1942

Stoughton completed the measurements on sample nos. 3 and 4 containing daughter $\mathrm{Pa}^{23}$ resulting from the decay of $\mathrm{Th}^{233}$ isolated on February 23 from bombardment no. Th+n-VII. He calculates that the ratio of electrons from $\mathrm{Pa}^{233}$ to those of $\mathrm{Th}^{233}$ is 1.82 for sample no. 3 , and 1.73 for sample no. 4. These are probably the best among the five determinations that have been made of this ratio.

He also measured the absorption of the $\mathrm{Pa}^{233}$ beta radiation in $3 \mathrm{mg} / \mathrm{cm}^{2}$ aluminum using Geiger-Mueller counter no. z-32 in order to determine the equivalent absorption of this radiation in the window of the counter (approximately $3 \mathrm{mg} / \mathrm{cm}^{2}$ mica thickness); he found that the ratio of the counting rate with $3 \mathrm{mg} / \mathrm{cm}^{2}$ Al to that without is 1.3 , indicating that this is the correction factor to allow for the absorption of $\mathrm{Pa}^{23}$ beta radiation in the window of Geiger-Mueller counter no. z-32.

Our University purchasing department forwarded our order for rental of the $1 \mathrm{gm}$ Ra-Be neutron source to the Canadian Radium and Uranium Corporation in New York today.

We received the $19 \mathrm{gm}$ of fast neutron bombarded uNH from washington University (bombardment no. $U, n-16$ ). Wahl is making an identical bombardment of a similar UNH sample on the Berkeley 60-inch cyclotron (bombardment no. U, n-17). Measurements will be made on both of these samples in the unseparated form, and by separation of element 93 fractions, to determine 
the relative yield of products produced in the st. Louis cyclotron as compared to the Berkeley cyclotron.

At the conclusion of each of the ether extractions of UNH Batches I, II and III, the UNH was recovered by water extraction from the ether and recrystallized in order to prepare it, after a suitable period of time for growth, for the recovery of $\mathrm{UX}_{1}$. This recovery has been carried on by Auerbach and Prestwood in their spare time as they have performed their other extractions and experiments. We now have on hand 500 pounds of such recovered UNH which has had sufficient time to allow a substantial fraction of its $\mathrm{UX}_{1}$ to grow in. We have found that with such recrystallized material it is not necessary to grind it to form small crystals to make it suitable for further ether extraction. This and our considerable experience now combine to make the extraction of this large amount of material a less formidable task than was the case when we started this work in January.

Today seven people are working on these 500 pounds (Batch IV) in room 301, each with his two liter pear-shaped separatory funnel, shaking the ether solution of UNH and separating the aqueous phases. These are Isadore Perlman, Fontana, Prestwood, Bonner, Auerbach, Adams and Americo Tanzillo, a technician who has joined our group and who has been working as Adams' assistant. Continuing at a good pace, they were able to finish the entire first extraction today. A recurring incident causes us considerable concern about the safety of handling so much flammable ether. Professor G. N. Lewis walks through the entire length of room 301 rather often, with his ever present lighted cigar in his mouth or hand, on his only available route to the darkroom that houses his spectrographic equipment. Nothing has happened so far but the fellows working with so much ether in this rather narrow room are understandably apprehensive.

Tuesday, March 10, 1942

Stoughton calculated a weighted average of the ratio of $\mathrm{Pa}^{233}$ electrons to $\mathrm{Th}^{23}$ electrons determined in experiments from bombardments $\mathrm{Th}+\mathrm{n}-\mathrm{III}$, -IV, -VI and -VII as 1.75. In the course of these determinations he has concluded that the best value of the half-life for $\mathrm{Th}^{233}$ is 23.5 minutes, rather than the 25.5 minutes that we have been using. Recalculated on this basis, the ratio becomes 1.66. Also from his value of the absorption half thickness (60 $\mathrm{mg}$ per sq. $\mathrm{cm}$ in $\mathrm{Al}$ ), he has calculated the small correction for the absorption of the $\mathrm{Th}^{233}$ beta radiation in the $A$ l window of GeigerMueller counter no. z-32 (a factor of 0.96), leading to a final corrected value of 1.59 for the ratio.

The second ether extraction of Batch IV was completed today, but unfortunately there was a good deal of crud in the aqueous phase, indicating perhaps the presence of some photodecomposition products of ether and UNH since much of the extraction was performed out on the porch in sunlight. 
Breslow and Hamaker have completed a successful experiment with the salicylaldehyde-o-phenylenediamine uranyl. About $10 \mathrm{gm}$ of the organic compound, together with about $20 \mathrm{mg}$ of uranyl acetate to act as carrier compound for the $\mathrm{U}^{237}$, were placed behind the beryllium target of the 60inch cyclotron and subjected to bombardment with neutrons (bombardment no. $\mathrm{U}, \mathrm{n}-18)$. The organic compound was then dissolved in $100 \mathrm{cc}$ of pyridine at room temperature and $700 \mathrm{cc}$ of water were added to precipitate the organic compound, which was filtered off. The solution was evaporated to dryness and the residue treated with concentrated nitric acid and finally evaporated so as to produce solid uranyl nitrate hexahydrate $\left(\mathrm{UO}_{2}\left(\mathrm{NO}_{3}\right)_{2}\right.$ $\left.\cdot 6 \mathrm{H}_{2} \mathrm{O}\right)$. This contained, in addition to any 6.8 -day $\mathrm{U}^{23}$ which might be present, most of the fission products, and it was therefore necessary to make a separation of the $\mathrm{U}^{237}$ from the fission products. This was accomplished by dissolving the uranyl nitrate hexahydrate in the minimum amount of pure, dry diethyl ether and separating the water phase; in this well established procedure a large percentage of the fission products go into the water and most of the uranium remains in the ether. In order to completely remove the fission products, it was necessary to go through this ether extraction process several times; the uranium nitrate in the ether was converted into solid uranyl nitrate hexahydrate, the starting point for every ether extraction, by extracting it from the ether with an excess of water and evaporating the water solution so as to produce solid $\mathrm{UO}_{2}\left(\mathrm{NO}_{3}\right)_{2}$ $\cdot 6 \mathrm{H}_{2} \mathrm{O}$. The final purified $\mathrm{UO}_{2}\left(\mathrm{NO}_{3}\right)_{2} \cdot 6 \mathrm{H}_{2} \mathrm{O}$, weighing some $20 \mathrm{mg}$, had an activity of $\mathrm{U}^{237}$ amounting to about 8 microcuries per 100 microampere-hours of bombardment. These results showed that a szilard-Chalmers concentration has taken place because under normal conditions, when a few grams of uranium salt are placed directly behind the beryllium target, the yield of $\mathrm{U}^{237}$ is about 0.05 microcuries per microampere-hour per gram of uranium element. However, because of the inconvenience of using pyridine, we have decided to look for a modification in the method which would eliminate the use of this substance.

A third ether extraction has been attempted on Batch IV with only partial success due to the sludge. The final aqueous phase from Batch IV was found by assay to contain about 15 millicuries of UX ${ }_{1}$ (indicating more than 50\% yield) and the solution of volume $1,000 \mathrm{cc}$ was turned over to Fontana and Bonner for the recovery of the $\mathrm{UX}_{1}$. The acid extracts from the various sludges will also be added to this in order to increase the yield.

Thursday, March 12, 1942

Wahl has completed his fission measurements with our 0.6 microgram of $93^{237}$ (Sample K) using our ionization chamber connected to a linear amplifier and recording system. The sample was placed directly on one electrode of the ionization chamber and as a neutron source he used our recently acquired $300 \mathrm{mg} \mathrm{Ra}-\mathrm{Be}$ mixture. The ionization chamber and $300 \mathrm{mg} \mathrm{Ra}-\mathrm{Be}$ neutron source were completely surrounded by paraffin. Under these conditions the 0.6 microgram $93^{237}$ sample gave 4 fission counts in about 56 hours of counting time. When the ionization chamber was surrounded by cadmium, there were recorded 2 counts in 56 hours of counting time. Thus the 
counting rate due to slow neutrons was 2 counts in 56 hours or 0.035 fission counts per hour. For a calibration experiment the 0.6 microgram sample of $93^{237}$ was replaced by 200 micrograms of electrolytically deposited natural uranium (containing 1.4 micrograms of $\mathrm{U}^{235}$ ), whose weight was determined by counting the alpha particles with our ionization chamber of calibrated efficiency. The easily measurable slow neutron fission rate of this uranium sample (273 counts in 23 hours, or 11.9 counts per hour), when compared to that for $93^{237}$, shows that the slow-neutron-fission cross section of $93^{237}$ is less than $1 \%$ of that for $U^{235}$.

Friday, March 13, 1942

Today Stoughton began to work up the residues from the Great Bear Lake pitchblende, upon which Morris Perlman has been working to isolate any 93 or 94 that might be present. He is starting with the solutions that contain HF. The purpose is to isolate the $\mathrm{Pa}^{231}$ so that we might have a sample to measure its slow and fast neutron fission properties in comparison with the other heavy isotopes with which we are working. He will use the knowledge of the chemistry of $\mathrm{Pa}$ that he has gained and will use zirconium carrier with zirconium phosphate precipitations in order to concentrate the $\mathrm{Pa}^{231}$. A concentration from zirconium will be achieved by a method of partial precipitation of zirconium iodate in which the $\mathrm{Pa}^{2} 31$ concentrates, a method which he has perfected in recent weeks. In the final stages the $\mathrm{Pa}^{231}$ will be further removed from impurities by precipitating with zirconium phosphate and the final sample of $\mathrm{Pa}^{231}$ will be obtained, after solution in a small volume, by electrolysis away from zirconium onto a copper plate.

Monday, March 16, 1942

Today Hamaker bombarded a sample of UNH (bombardment no. U,n-19) with the neutrons from the 60-inch cyclotron in order to produce tracer $93^{239}$ for our group and acquaint himself with the chemistry of 93 . He isolated the $93^{239}$ by our conventional oxidation-reduction procedure using $\mathrm{BrO}_{3}{ }^{-}$as oxidizing agent and $\mathrm{SO}_{2}$ as reducing agent, and rare earth fluoride as carrier.

Fontana and Bonner completed the extraction of the UX 1 fraction from Batch IV. They diluted the starting solution to 4 liters, added $350 \mathrm{mg}$ of $\mathrm{La}^{+++}$and $30 \mathrm{mg}$ of $\mathrm{Ce}^{+++}$, and then added $\mathrm{HF}$ to precipitate the rare earth fluorides (there was no thorium present) from this solution which was $0.5 \mathrm{M}$. in hydrogen ion. They then carried it through the usual procedure of fluoride reprecipitations, concentration of $\mathrm{UX}_{1}$ by separating the $\mathrm{Ce}^{++++}$from the $\mathrm{La}^{+++}$by precipitation of the iodate, precipitation of $\mathrm{CeF}_{4}$ and reprecipitations to remove the last vestiges of uranium, then solution in acid; they set aside the final solution to allow the $\mathrm{Ux}_{1}$ to decay to the daughter $\mathrm{U}^{234}$. 
The beta decay of sample Th+n-I-Pa-III as followed on our FP-54 ionization chamber indicates a half-life of 27.4 days for $\mathrm{Pa}^{233}$. The growth of $\mathrm{U}^{233}$ alpha particles in this sample as measured with the ionization chamber-linear amplifier-magnetic field outfit $(W-1)$ is consistent with a half-life of 27.4 days for the parent beta emitting $\mathrm{Pa}^{233}$, and extrapolates to a total at infinite time of 428 alpha counts per minute after the constant contribution of $\mathrm{Pa}^{231}$ alpha particles is subtracted. From this disintegration rate of daughter $U^{233^{3}}$ alpha particles and the disintegration rate of parent $\mathrm{Pa}^{233}$ beta particles measured on December 4, 1941, corrected to zero time, it is now possible to calculate the half-life of $\mathrm{U}^{233}$. Correcting for the geometry factor of the ionization chamber-linear amplifier-magnetic field setup $(\mathrm{W}-1)$, which has been determined to be 0.019 (by counting a small sample of $\mathrm{U}^{233}$ both in this ionization chamber and our calibrated "inside" ionization chamber, $(-3)$, we can calculate that the total $\mathrm{U}^{23}$ disintegration rate is 375 alpha particles per second. The $\mathrm{Pa}^{23}$ the galvanometer deflection measured with our ionization chamber FP-54 electrometer on December 4, 1941, corrected for absorption and decay, is $1795 \mathrm{~mm}$ (standard conditions of R5S5) corresponding to the time of last separation of $\mathrm{Pa}^{23}$ from daughter $\mathrm{U}^{23}$ (11:59 p.m., November 17, 1941). Using this value, the calibration for $\mathrm{Pa}^{233}$ radiation of March 5, 1942 $\left(0.058 \mathrm{~mm}\right.$ of galvanometer deflection per microcurie of $\left.\mathrm{Pa}^{233}\right)$, and correcting for the backscattering of $\mathrm{Pa}^{23}$ beta particles from copper relative to cellophane (factor of 0.67) and from cellophane (factor of 0.97), for the absorption of $\mathrm{Pa}^{233}$ radiation in the mica window of the Geiger-Mueller counter used in the calibration of the FP-54 ionization chamber (factor of 1.3 ), and for the conversion electrons in the $\mathrm{Pa}^{233}$ radiation (factor of 1.6), we have

\section{$\frac{1795 \times 0.67 \times 0.97 \times 1.3}{0.058 \times 1.6} \cong 16,300$ microcuries or 16.3 millicuries in}

sample no Th+n-I-Pa-III. Since one microgram of $27.4-$ day $\mathrm{Pa}^{233}$ corresponds to 20.5 millicuries, we can calculate the weight of $\mathrm{U}^{233}$ in sample $\mathrm{Th}+\mathrm{n}-\mathrm{I}-$ Pa-III after all of the parent $U^{233}$ has decayed as

$$
\frac{16.3}{20.5}=0.80 \text { micrograms }
$$

From these data we can now calculate the alpha half-life of $\mathrm{U}^{233}$ as

$$
\frac{27.4 \times 16.3 \times 3.7 \times 10^{7}}{365 \times 375}=1.20 \times 10^{5} \text { years. }
$$

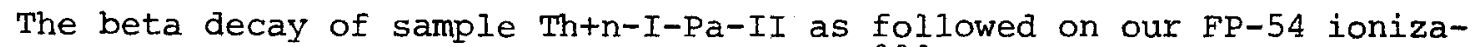
tion chamber also indicates a half-life for $\mathrm{Pa}^{233}$ of 27.4 days. The growth of $\mathrm{U}^{233}$ alpha particles in this sample as measured with the ionization chamber-linear amplifier-magnetic field outfit $(W-1)$ also corresponds to a half-life of 27.4 days for the parent beta-emitting $\mathrm{Pa}^{233}$; the alpha intensity extrapolates to about 75 per second at infinite time. Considerations similar to those for sample $\mathrm{Th}+\mathrm{n}$-I-Pa-III indicate an initial intensity of $\mathrm{Pa}^{233}$ beta particles of 3.0 millicuries which, together with the intensity of alphas from daughter $\mathrm{U}^{233}$ (75 per second), also leads to an 
alpha half-life of close to $1.2 \times 10^{5}$ years for $\mathrm{U}^{233}$. The beta decay of $\mathrm{Pa}^{233}$ samples isolated on December 23, 1941 from bombardment $T h+n-I I$, measured with our electroscope no. 9, also show a half-life of 27.4 days; the error corresponding to an average of all these determinations is \pm 0.2 days-i.e., the half-life of $\mathrm{Pa}^{233}$ is $27.4 \pm 0.2$ days.

This evening I began dictating to Helen in her apartment the report "Chemical Properties of Elements 94 and 93 " using material provided by Wahl in connection with the writing of his thesis. This will describe all of our work on the chemical tracer properties of elements 94 and 93, including the oxidation experiments and the experiments determining carrying by various precipitates.

Tuesday, March 17,1942

During the last week Fontana has been working on the Brown Mud residue that we received from the Shattuck Chemical Company as a result of the visit and work of Joseph G. Hamilton at their plant in Denver late last. month and early this month, during which he put this material in shape for shipment to us. There are indications that this concentrate will contain more $\mathrm{UX}_{1}$ than corresponds to the weight of the uranium present because the $\mathrm{UX}_{1}$ accurnulates in these residues during the shattuck Chemical Company UNH crystallization process.

I learned today that Frank Spedding has set March 26 as the date for the start of his visit to Berkeley and that he will be accompanied by his colleague, Dr. Iral B. Johns, Jr.

I therefore wrote a letter to Dr. Spedding at his Department of Chemistry, Iowa State College, Ames, Iowa address saying that I am looking forward to his and Dr. Johns' arrival in Berkeley on March 26.

This evening I continued to dictate to Helen the report "Chemical Properties of Elements 94 and 93."

Wednesday, March 18,1942

Today Gofman performed an experiment with 50-year 94 tracer and 2.3-day $9.3^{239}$ tracer to investigate whether 94 forms a volatile tetraoxide as does its homolog osmium. He used $\mathrm{S}_{2} \mathrm{O}_{8}^{-2}$ and $\mathrm{Ag}^{+}$as his oxidizing agent and added a few milligrams of osmic acid as carrier. He then distilled the osmium tetraoxide from the solution, catching the distillate in sodium hydroxide solution; he acidified the distillate, added $\mathrm{SO}_{2}$ as a reducing agent, and precipitated $\mathrm{LaF}_{3}$ which he found contained none of the 94 or 93 tracer. He reduced with $\mathrm{SO}_{2}$ the original solution from which the distillation had been made and precipitated $\mathrm{LaF}_{3}$ which he found to contain essentially all of the 94 and 93 tracer. Hence, this experiment proves that under these conditions, at least, 94 does not form a volatile tetraoxide. 
I received a letter dated March 15, 1942 from M. Pochon in response to my letter to him of February 25, 1942. He said that the shipment of the 200 pounds of UNH was made on March 11 and that the salt is about three months old in line with my request. He also indicated that he has shipped to us yesterday a liter of mother liquor as I had requested.

I received a letter from Harold C. Urey dated March 16, 1942 informing me that he had been talking recently to Compton in regard to volatile fluorine compounds of 93 and 94 and that it was his (Urey's) impression that we have had some difficulty in preparing UF ${ }_{6}$. He pointed out that his group at Columbia University was completely equipped to prepare $U_{6}$ and wondered if I would like to have his group prepare some for our use at Berkeley.

In our informal nuclear seminar John Hamaker reported on chemical behavior of extremely small amounts of material, and Dr. Stoughton reported on the paper "Photodisintegration of Beryllium and Deuterium" (F. E. Myers and L. C. Van Atta, Phys. Rev. 61, 19 [1942]).

This evening I completed my dictation to Helen of the report "Chemical Properties of Elements 94 and 93." Besides the discussion of the tracer chemical properties, a section on yields of $93^{235}$ or ${ }^{238}$ (and thus of $94^{235}$ or ${ }^{238}$ ) from deuteron bombardments and of $93^{239}$ (and thus of $94^{239}$ ) from neutron and deuteron bombardments is included. This paper, under the authorship of wahl and me, also includes for the first time the suggestion that element 94 be given the name plutonium (symbol Pu) after Pluto, the second planet beyond Uranus, in line with McMillan's suggestion that element 93 be given the name neptunium (symbol $\mathrm{Np}$ ) after Neptune, the first planet beyond Uranus, from which uranium derives its name. We also point out that there is practically no resemblance in chemical properties between neptunium and plutonium on the one hand and rhenium and osmium on the other. We indicate that the chemical properties of neptunium and plutonium are those of a rare-earth-type group of elements starting at the upper end of the Periodic Table and go on to suggest that the group can be just as well considered to be starting with actinium or thorium as with uranium, which has been previously suggested as the starting point.

I also started my dictation to Helen of the report "Production and Properties of 50-Year Element 94" using material provided by wahl and Kennedy. This report will be authored by Wahl, Kennedy and me and will describe the production and nuclear properties of our 50-year element 94 isotope (for which we now think the best assignment is $94^{235}$ or $94^{238}$ ).

Thursday, March 19, 1942

This evening I completed dictating to Helen our report "Production and Properties of 50-Year Element 94." The report indicates that our best value for the half-life is 50 years and the range of the alpha particles as determined by absorption in air and in aluminum corresponds to $4.1 \pm 0.1$ $\mathrm{cm}$ in air at standard conditions $\left(15^{\circ} \mathrm{C}\right.$. and $\left.760 \mathrm{~mm}\right)$. We also describe 
properties of the 2.0 -day $93^{235}$ (or $93^{238}$ ) beta-particle-emitting parent of the 50-year 94; the half-life is 2.0 days and absorption measurements indicate an upper energy limit for the beta particles of $1.0 \mathrm{Mev}$ and gamma rays of energy $1.1 \mathrm{Mev}$. Included is a summary of the yields of the 2.0-day 93 isotope and of the $2.3-$ day $93^{239}$ produced in the Berkeley 60-inch cyclotron by the bombardment of uranium with $16 \mathrm{Mev}$ deuterons.

Helen completed the typing of the report "Chemical Properties of Elements 94 and 93" authored by Wahl and me and it bears today's date.

Friday, March 20, 1942

Morris Perlman has completed his meticulous isolation of the 94- and 93-containing fraction from the dissolved pitchblende which he started on early in December. He began by adjusting the acidity of each of his nine starting solutions somewhere in the range of $0.5 \mathrm{M}$. to $3 \mathrm{M}$. , then added sulfur dioxide to assure that any 94 or 93 would be in the reduced fluoride insoluble form, added lanthanum and cerium carrier when necessary, and then added hydrofluoric acid to each and removed the fluoride precipitates.

The various fluoride precipitates were all combined and dissolved in concentrated sulfuric acid, and, after dilution with water and the addition of dilute nitric acid in order to oxidize any uranous uranium to the uranyl form, the rare earth fluorides were again precipitated by the addition of hydrofluoric acid. This rare earth and thorium fluoride precipitate, which amounted to about 20 grams and which would contain, in their reduced forms, any 94 or 93 which might have been present in the original pitchblende, was dissolved in sulfuric acid and reprecipitated as the fluoride.

It was now necessary to go through a chemical procedure designed to isolate any such 94 or 93 into a very thin layer $\left(0.3 \mathrm{mg} / \mathrm{cm}^{2}\right.$ ) of rare earth carrier material, taking special care to eliminate all the elements in the uranium, thorium and actinium radioactive series which would interfere with the detection of the 94 or 93 in the final sample. To do this the 20-gram rare earth plus thorium fluoride precipitate was dissolved in sulfuric acid and after the addition of a few grams of potassium peroxydisulfate $\left(\mathrm{K}_{2} \mathrm{~S}_{2} \mathrm{O}_{8}\right)$ and a few tenths of a gram of silver nitrate, hydrofluoric acid was added to again precipitate the rare earth and thorium fluoride. In this procedure the 94 and 93 remain in solution, present in their higher oxidation states. This solution, after the removal of the fluoride precipitate by centrifugation, was boiled in order to remove the hydrofluoric acid and decompose the peroxydisulfate and was then treated with sulfur dioxide in order to reduce the 94 and 93 to their lower, fluoride insoluble, oxidation states. About 120 milligrams of lanthanum and cerium carrier were then added and the fluoride precipitated by the addition of hydrofluoric acid.

This 120-milligram fluoride precipitate was then dissolved in sulfuric acid and taken through another identical cycle in which the amount of rare earth was reduced to about 5 milligrams. These 5 milligrams of rare earth, which would contain the 94 or 93 , were then dissolved in sulfuric acid and, after the addition of peroxydisulfate and silver ion, the rare earths were 
removed from solution by precipitation as the fluoride. The hydrofluoric acid was removed by boiling the solution until the white fumes of sulfur trioxide appeared and after dilution another 0.2 milligram of rare earth carrier was added. After treatment with peroxydisulfate and silver ion, this 0.2 milligram of rare earth was precipitated as fluoride. The purpose of this precipitation, which was made just before the final isolation of the material which would contain the 94 and 93 , was to establish that there was a negligible amount of isotopes of thorium (UX ${ }_{1}$, Io) present in the solution at this stage. This 0.2 milligram showed no Detectable beta activity above the background of the Geiger-Mueller counter, and an alpha counting rate of about 45 per hour when placed on one electrode of our ionization chamber $(w-3)$ in which the calibrated counting efficiency is about 45 \% .

The hydrofluoric acid was removed from the remaining solution by boiling until the dense white fumes of sulfur trioxide appeared and, after dilution and reduction with sulfur dioxide, another 0.2 milligram of rare earth was added. This was precipitated as fluoride by the addition of hydrofluoric acid, and centrifuged onto a platinum disk for final measurements. This sample should contain any 94 or 93 which was present in the original pitchblende. (Calibration experiments, starting with 20 grams of rare earth and ending with 0.2 milligram of rare earth fluoride in an identical chemical procedure, in which 50-year alpha emitting 94 was added as tracer to the original 20 grams, proved that the yield of 94 in this rather lengthy chemical procedure amounted to about $80 \%$. Earlier careful counting experiments proved that the rare earth carrier material and the platinum, upon which the final sample was mounted, were free from alpha-emitting contamination.)

Helen completed the typing of our report "Production and Properties of 50-Year Element 94" and it bears today's date.

Saturday, March 21, 1942

Wahl has completed the comparison measurements on the fast neutron bombardments made at the St. Louis and Berkeley cyclotrons earlier this month and concludes that the yield of $93^{239}$ at Berkeley is about twice that of st. Louis under comparable, conditions.

Today Gofman started to recover the remaining $94^{239}$ from all the residues of our bombaraments no. 93-18 (June 5-21, 1941) and no. U,n-15 (March 1911). These were the large neutron bombardments, $1.9 \mathrm{~kg}$ UNH and 14,000 microampere-hours of deuterons (bombardment no. 93-18) and $1.2 \mathrm{~kg}$ UNH with 15,000 microampere-hours of deuterons (bombardment no. U, n-15), and thus there should remain substantial guantities of $94^{239}$ in these residues. His plan is to take all the existing rare earth fluoride precipitates, add the rare earth fluorides precipitated from various aqueous solutions, dissolve and combine them, and then put them through oxidation-reduction procedures to finally isolate the $94^{239}$. 
Today I mailed to Dr. Lyman J. Briggs, Chairman of Section S-1, copies of our reports "Chemical Properties of Elements 94 and 93" by Wahl and me, and "Production and Properties of 50-Year Element 94" by Wahl, Kennedy and me. I also mailed copies of these reports to Professor Arthur H. Compton, Director of the Metallurgical Project at the University of Chicago, and Eger $V$. Murphree, member of section S-1, to his address at the Standard Oil Development Company in New York City. In addition, I mailed copies of the two reports to Professor Harold C. Urey, member of Section S-1, to his address in the Department of Chemistry, Columbia University, and included an extra copy of "Chemical Properties of Elements 94 and 93" for him to forward to Professor Charles A. Kraus of Brown University, who is working in connection with the Metallurgical project on uranium chemistry. In my letter to Urey I tried to allay his fears that we were having trouble in studying the fluorides of elements 94 and 93 , indicating that we have not yet started this work.

NOTE: The paper "Chemical Properties of Elements 94 and 93" by G. T. Seaborg and A. C. Wahl was issued immediately (under the issue date of March 19, 1912) by the S-1 Committee in Washington with the report number A-135. After the war it was published in J. Am. Chem. Soc. 70, 1128 (1948).

"Production and Properties of 50-Year Element 94 " by G. T. Seaborg, A. C. Wahl and J. W. Kennedy was issued by the S-I Committee as report number A-136 under the date of March 20, 1942. It was also published, under the changed title "Nuclear Properties of $94^{238}$ and $93^{238}$ " by G. T. Seaborg, A. C. Wahl and J. W. Kennedy in National Nuclear Energy Series, Division IV-Plutonium Project Record, Volume 14B, The Transuranium Elements -Research Papers (edited by G. T. Seaborg, Joseph J. Katz and Winston L. Manning) as Paper No. 1.4. Most of the early papers were published in this NNES volume but references will be made here to this form of publication only when papers do not appear as well in regular journals.

Sunday, March 22, 1942

Today Gofman, using some of Hamaker's $93^{239}$ tracex (isolated last Monday) completed a 13 hour electrolysis experiment fxom an oxidizing solution of $\mathrm{BrO}_{3}^{-}$and in the presence of $0.3 \mathrm{M}$. acetate ion. He found a yield of about $84 \%$ on the electroplate. 
Norman Hilberry, who is looking after interlaboratory relations for Compton on the Metallurgical Project, arrived yesterday to spend the week with us discussing our work at Berkeley. Today he visited with Kennedy, Wahl, English, Friedlander, Gofman and me. Kennedy described his process of mica splitting to produce thin, uniform sheets of mica (about $3 \mathrm{mg} / \mathrm{cm}^{2}$ thickness) for use as Geiger counter windows. I described our investigations on the chemistry of elements 94 and 93, which I indicated as having top priority, and our results on the nuclear properties of $94^{239}$ and the 50 -year $94^{235}$. I indicated that the yield of $94^{239}$ in test samples of UNH bombarded with fast neutrons at the St. Louis cyclotron and the Berkeley cyclotron show a substantially larger yield at Berkeley. I noted that Wahl, English and Gofman are working on these problems.

I also discussed with Hilberry our current work on the investigation of the nuclear properties of $\mathrm{U}^{233}$ and $93^{237}$, indicating that this work is being carried out by Gofman (on $\mathrm{U}^{233}$ ) and wahl (on $93^{237}$ ). I also described our work on the isolation of $\mathrm{U}^{234}$ through the separation of $\mathrm{UX}_{1}$ from large amounts (500 to 1,000 pounds) of UNH for the purpose of measuring the fission properties of $\mathrm{U}^{23 \frac{4}{4}}$. I said that this work is being carried out by Isadore Perlman, B. J. Fontana and Joseph G. Hamilton. I also described our search for elements 94 and 93 in various uranium and rare earth ores, indicating that this is being done by Morris Perlman and Wahl, and our work on the szilard-Chalmers process for concentrating $\mathrm{U}^{237}$ from neutron-bombarded uranium being done by David S. Breslow.

Following these discussions Hilberry, Kennedy, Martin Kamen and I had lunch together at the Faculty Club. After lunch I described my present status at Berkeley; that is, on leave of absence from the University of California and working on these numerous investigations supported by OSRD. I indicated that I could move with some of my people to Chicago if this were desired. He said that I should plan to do so. I also described the roles of Wahl, Kennedy and Segrè, pointing out that Kennedy is now working with Segrè on the $U^{235}$ aspect of the general problem.

Today we received a liter of mother liquor from the Port Hope Refinery that $M$. Pochon has shipped to us in response to my request of February 25, 1942. Assays will be made of this to determine the feasibility of this as a more concentrated source of $\mathrm{UX}_{1}$.

Tuesday, March 24, 1942

Now that the weight of $\mathrm{U}^{233}$ in sample no. Th+n-I-Pa-III (corrected for incomplete decay of $\mathrm{Pa}^{233}$ ) is known, it is possible to determine the weight of $\mathrm{U}^{233}$ in Sample $\mathrm{J}$ by determining the ratio of the alpha activity in the two samples. Using the ionization chamber-linear amplifier-magnetic field setup (W-1) and a screen to cut down the intensity of alphas in order to avoid coincidence corrections, Gofman and Stoughton measured the alpha count of Sample J as 640 per minute and of sample Th+n-I-Pa-III (corrected to infinite time) as 131 per minute (after subtracting the contribution of $\left.\mathrm{Pa}^{231}\right)$. Thus, sample $J$ contains 
$\frac{640}{131}=4.9$ as much $U^{233}$ as does $T h+n-I-P a-I I I$. The weight of $U^{23}$ in Sample $J$ is therefore $4.9 \times 0.8$, or actually

$$
\frac{4.9 \times 16}{20.5}=3.8 \text { micrograms. }
$$

Wednesday, March 25, 1942

Morris Perlman has concluded his counting measurements on the final $0.2 \mathrm{mg}$ of rare earth fluoride precipitate isolated from the $400 \mathrm{gm}$ of pitchblende in a procedure which was completed last Friday. This final sample shows no detectable beta activity above the background of the GeigerMueller counter. It does, however, show an alpha counting rate of about 90 per hour when placed on one electrode of our ionization chamber $(\mathrm{W}-3)$ in which the calibrated counting efficiency is about 45\%. This alpha counting rate might very well be due to the presence of $94^{239}$ in view of the expectation that some of this isotope be present as the end product formed as the result of absorption of spontaneous fission neutrons by $\mathrm{U}^{238}$. The chemicaI procedure is very stringent and specific for the isolation of 94 (and 93). A counting rate of about 90 per hour, amounting to some $10^{-6}$ microcurie, corresponds to the order of $10^{-5}$ microgram of 30,000 -year $94^{239}$, equivalent to something like one part in $10^{14}$ of $94^{239}$ in the oxiginal pitchblende concentrate. This amounts to only a few percent of the amount to be expected if a large proportion of the spontaneous fission neutrons were absorbed by the $U^{238}$, but this is not surprising in view of the number of other neutron absorbing materials which might be present in the pitchblende.

We received today the 200 pounds of triply recrystallized uNH which we have ordered from the Shattuck Chemical Company in Denver (Batch V).

Thursday, March 26, 1942

Spedding and Johns are visiting with us today as scheduled. I gave them a complete description of our research program and a tour of our laboratories in which they met the people involved. Spedding asked that I prepare for Compton a list of proposed chemical investigations on element 94 and other work that might be carried out at the Metallurgical Laboratory in Chicago and at Berkeley, which I indicated I would do.

Spedding and I had a discussion of the plans, discussed with Hilberry, for my move to the Metallurgical Laboratory and we agreed that this move should take place in the near future.

Hilberry had a final session with Kennedy to learn more about the details of the program of Kennedy and segrè for measuring the fission properties of $\mathrm{U}^{235}$ and $94^{239}$. 
Gofman has finished his tests for slow and fast neutron fission of any isotopes of 94 and 93 that might be present in Morris Perlman's final fraction isolated from the $400 \mathrm{gm}$ of pitchblende. Since it is likely that any isotopes of 94 or 93 would undergo fission with slow or fast neutrons, a test for fissions with neutrons was made by placing the final sample on one electrode of his ionization chamber connected to a linear amplifier and recording system adjusted to respond to the impulses due to fissions. When slow neutrons were used, from our $300 \mathrm{mg}$ Ra-Be source with paraffin between the chamber and neutron source and around the chamber and neutron source, there was recorded zero fissions in 7 hours of counting. When the sample was replaced by a "standard" 200 microgram uranium sample, containing therefore 1.4 micrograms of $\mathrm{U}^{235}$, the fission counting rate due to slow neutrons amounted to about 15 counts per hour. Therefore, assuming that the slow neutron fission cross section of the 94 or 93 would be of the same order of magnitude as that of $\mathrm{U}^{235}$, there was present in the final sample no more than a small fraction of a microgram of any isotope of 94 or 93 capable of undergoing fission with slow neutrons. So far as fast neutrons are concerned, our experiments place an upper limit of the order of a microgram on the amount of any isotope of 94 or 93 which was present and capable of undergoing fission with fast neutrons; therefore, we can say that there was not present in this pitchblende as much as one part of 94 or 93 in $10^{8}$ or $10^{9}$ parts of ore.

Today Isadore Perlman and Fontana completed their isolation of the $\mathrm{U}^{234}$ from the $\mathrm{UX}_{1}$ samples corresponding to UNH Batches I, II and III which had been set aside to allow their $\mathrm{UX}_{1}$ to decay to daughter $\mathrm{U}^{234}$. After removal of the $\mathrm{Th}$ and $\mathrm{Ce}^{+4}$ by repeated fluoride precipitation, the $\mathrm{U}^{234}$ was isolated by electrolysis, using a procedure which they have perfected during the last few weeks based on Gofman's electrolysis procedure for the isolation of $\mathrm{U}^{233}$. The electrolysis was performed from a solution $0.1 \mathrm{M}$. in $\mathrm{KF}$ and about $0.01 \mathrm{M}$. in acetate, and the $\mathrm{U}^{234}$ was deposited on a platinum plate which served as a cathode. The $U^{234}$ plated out nearly quantitatively and the plate has a dark brown, well-adhering film-probably platinum of entirely negligible weight (less than $0.1 \mathrm{mg} / \mathrm{cm}^{2}$ ). An assay of the weight of the $\mathrm{U}^{234}$ was made by counting the alpha particles. The alpha counting rate in ionization chamber $W-3$, corresponding to a decay rate of 130 alpha particles per minute, together with the half-life of $U^{234}$ of about $2.7 \times 10^{5}$ years, indicates the presence of 0.1 micrograms of $\mathrm{U}^{234}$ in our final sample. This will be used by Gofman for the determination of the slow neutron fission cross section of $\mathrm{U}^{234}$.

Lawrence met with spedding in order to discuss my impending move to the Metallurgical Laboratory and there was agreement that Isadore Perlman and I should move to Chicago in the very near future. They also agreed that it can be determined later whether others from my group here will join me at Chicago and whether certain phases of the work here might continue in close collaboration with our work at Chicago. They agreed that wahl should continue his studies of the chemistry of 94 at Berkeley, working in close liaison with the Chicago group.

During the last few days Joe Hamilton has been helping Fontana on the extraction of UX, from the Denver Brown Mud. 
We received the $1 \mathrm{gm}$ Ra-Be neutron source that we ordered on March 9 from the Canadian Radium and Uranium Corporation in New York.

Saturday, March 28,1942

Professor Lawrence wrote to Arthur Compton at Chicago describing the plans for Isadore Perlman and me to move to Chicago in the very near future, with the determination of the plans for the others to be worked out later, other than wahl who will remain in Berkeley. Lawrence also suggested that the negotiations for the use of the St. Louis cyclotron proceed as quickly as possible in order to increase the production of $94^{239}$. He indicated that the Berkeley 60-inch cyclotron will add a night shift in order to increase its production.

Monday, March 30, 1942

233 Today Gofman completed his measurements on the spontaneous fission of $\mathrm{U}^{2.33}$ using Sample $\mathrm{J}$ with the apparatus set up in his apartment on February 25.

After overcoming a number of difficulties with the stability of his amplifier circuits, he has managed to conduct 243 hours of meaningful operation during which he has observed no spontaneous fission counts with the 3.8 micrograms of $\mathrm{U}^{233}$ in sample $\mathrm{J}$. In order to determine the efficiency of his ionization chamber and amplifier outfit for detecting spontaneous fission events, he placed a sample of natural uranium on the electrode of his ionization chamber and then measured the variation of the neutron-induced fission rate with amplifier gain. Also taking into account the geometry of the ionization chamber, he finds that the overall efficiency of the apparatus for recording spontaneous fissions amounts to about $72 \%$. Hence, his 243 hours of counting corresponds to $243 \times 0.72=175$ hours of effective counting during which no spontaneous fission events were recorded. Since the expected average interval between fission counts for a half-life of $10^{14}$ years corresponds to 135 hours, it seems probable that the half-life for the spontaneous fission of $\mathrm{U}^{233}$ is of the order of, or greater than, $10^{14}$ years.

Today we received 200 pounds of UNH from the Port Hope Refinery as promised by M. Pochon's letter of March 15, 1942 and 50 pounds of UNH from the Mallinckrodt Company in St. Louis, a new source of UNH as a result of their impending role in producing pure uranium for the various pile experiments underway at the Metallurgical Laboratory at the University of Chicago. We refer to this combined batch of 250 pounds of UNH as Batch VI. 
During experiments started the day before yesterday and completed today, Gofman measured the slow neutron fission cross section of $\mathrm{U}^{234}$ using the 0 . II microgram sample prepared by Isadore Perlman, Fontana, et al., via the extraction of $U_{1}$ from uranium and subsequent beta decay to $U^{234}$. He used the 300 $\mathrm{mg}$ Ra-Be neutron source and made comparisons with a standard 200 microgram natural uranium sample (U-St-10) containing 1.4 micrograms of $\mathrm{U}^{235}$. He has found with the 0.1 microgram $U^{234}$ sample 17 fission counts in 24 hours of counting time in the presence of paraffin without a cadmium absorber; when the ionization chamber was surrounded by cadmium, there were obtained 6 fission counts in 33 hours of counting time. The fission counting rate of the $\mathrm{U}^{234}$, due to slow neutrons, was therefore about 0.5 counts per hour. When the $U^{234}$ sample was replaced by the standard uranium sample, the slow neutron fission counting rate measured under identical conditions was about 12 counts per hour. Therefore, the ratio of the slow neutron cross section of $\mathrm{U}^{234}$ relative to that of $\mathrm{U}^{235}$ is

$$
\frac{0.5}{12} \times \frac{1.4}{0.1}=0.5
$$

This should probably be considered as an upper limit because contamination of the $U^{234}$ sample by as little as 8 micrograms of natural uranium would be sufficient to account for the whole counting rate. Larger samples of $\mathrm{U}^{234}$ should be available within a few months so that it will be possible to make more accurate measurements at that time.

Gofman used 50-year tracer from English's bombardment no. U,D-2 to investigate the electrolytic deposition of 94 from oxidized solution. He used $\mathrm{S}_{2} \mathrm{O}_{8}^{-2}$ and $\mathrm{Ag}^{+}$as oxidizing agent. He had difficulty maintaining a high percentage of the 94 in the oxidized form under the electrolysis conditions, but of that in the oxidized form a large percentage was electrolytically deposited.

Bonner, Prestwood and Auerbach made the first ether extraction on Batch $\mathrm{V}$ for which the history indicates that only about one-fourth of the $\mathrm{UX}_{1}$ has grown in. This is our best batch to date from the standpoint of impurities, undoubtedly due to the fact that it was triply recrystallized, and thus they were able to complete the first extraction in a record time of $6-1 / 2$ hours.

I wrote to Professor Compton at Chicago enclosing a list of proposed chemical investigations on element 94 to be carried to completion as soon as possible here at Berkeley and at Chicago. I indicated that I plan to arrive in Chicago by April 20th and that I will bring a few men with me then or immediately afterwards, while leaving the remainder of my group at Berkeley until the course of events determines the most efficient arrangement. I also called attention to Gerhart Friedlander, who has completed his Ph.D. work with me this spring, pointing out that he is an alien who will receive his final naturalization papers soon, and hence should be incorporated officially in the chemical program at that time; I said he has contributed heavily in our program here but that I have been forced to keep him in the background due to his alien status. I also indicated that we are constructing some of the most vital and specialized equipment for shipment to Chicago in order that we may get underway as soon as possible after our arrival. 
I enclosed in my letter to Compton a summary entitled "Additional Investigations Underway by My Group at Berkeley" and a summary of "Investigations Which Might Be Considered in Case A Very Large Chemical Program Were Undertaken" as follows:

\section{ADDITIONAL INVESTIGATIONS UNDERWAY BY MY GROUP AT BERKELEY}

"1. Investigation of radioactive and fission properties of $U^{233}$ (nearing completion).

"2. Investigation of radioactive and fission properties of $93^{237}$ (nearing completion).

"3. Measurement of fission properties of $U^{234}$. The $U^{234}$ is prepared by extracting uranium-free $U_{1}$ from uranyl nitrate hexahydrate, of which there is now on hand about 900 pounds which has been purified by the ether extraction method. We may have about one microgram of $\mathrm{U}^{234}$ in about two months, and smaller amounts, sufficient for rough measurements, may be available sooner.

"4. Search for 94 and 93 in natural minerals. A large number of likely minerals have been gathered and are on hand in Berkeley.

"5. Development of a Szilard-Chalmers procedure for the concentration of 23-minute $\mathrm{U}^{239}$ and 7-day $\mathrm{U}^{237}$ formed in the neutron bombardment of uranium. Organic uranium compounds have been used, and the experiments have been successful on a small scale and now are being extended for largescale work. Seven-day $U^{237}$ of extremely high specific activity will be useful as a tracer for microgram amounts of uranium. (We are now using $\mathrm{U}^{233}$ for this purpose, but it is rather expensive and laborious to prepare.)

"6. A more accurate determination of the half-life of $94^{239}$, and hence a more accurate determination of the weight of $94^{239}$ in our samples, in order to obtain more accurate values for the fission properties of $94^{239}$ (nearing completion)."

INVESTIGATIONS WHICH MIGHT BE CONSIDERED IN CASE A VERY LARGE CHEMICAL PROGRAM WERE UNDERTAKEN

"1. The separation of $\mathrm{UX}_{1}$ during the purification of the large amounts of uranium which is occurring at port Hope, Ontario. The isolation of about 20 micrograms of $\mathrm{U}^{234}$ should be possible in case it seems worthwhile to make a thorough investigation of the spontaneous fission rate of $\mathrm{U}^{234}$.

"2. The development of chemical methods for separating kilograms of $\mathrm{U}^{233}$ from large amounts of thorium and fission products in case $\mathrm{U}^{2.33}$ should prove to be a useful isotope.

"3. Experiments to determine with certainty the isotopic assignment for the 50-year 94. A determination of the variation of the yield with the energy of the bombarding deuterons should establish that the 50-year 94 is formed in a $\underline{d, 2 n}$ or $\underline{d, n}$ reaction. To check on our tentative assigniment of the 50-year 
94 to $94^{235}$, a search for $93^{238}$ from deuteron-activated uranium might be made. (Preliminary work on the latter has begun in Berkeley.)

"4. A microchemical investigation of the chemical properties of 94 , using pure $94^{239}$ in the absence of carrier, after the preparation of some 20 or 30 micrograms of $94^{239}$.

"5. Measurement of the fission properties of the 2.3-day $93^{239}$. Preliminary investigations at Berkeley show that this will be very difficult and will require a linear amplifier of very high resolving power because of the extremely high beta-activity of the $93^{239}$ in microgram amounts.

"6. Search for new long-lived fission products in the extremely strong neutron-activated samples, from which $94^{239}$ has been isolated, in order to obtain a more accurate determination of the distribution of fission products in an extension of Fermi and Anderson's work.

"7. A search for $\mathrm{U}^{232}$ formed in the deuteron bombardment of uranium from the reactions $\mathrm{Th}^{232}(\mathrm{~d}, 2 \mathrm{n}) \mathrm{Pa}^{232}$ and $\mathrm{Pa}^{232} \mathrm{~B}^{-} \mathrm{U}^{232}$, in the hope that $\mathrm{U}^{232}$ might be a better tracer for microgram amounts of uranium than are $\mathrm{U}^{233}$ or $\mathrm{U}^{237}$.

"8. Search for an alpha-emitting $U^{236}$ formed from $U^{235}(d, p) U^{236}$, in the hope that $\mathrm{U}^{236}$ might be a good tracer for microgram amounts of uranium.

"9. A quantitative determination with an electron spectrometer of the number of conversion electrons in the radiation of 23 -minute $U^{239}, 2.3$-day $93^{239} ; 24$-minute $\mathrm{Th}^{233}, 27-\mathrm{day} \mathrm{Pa}^{233} ; 7-\mathrm{day} \mathrm{U}^{237}$; in order to weigh more accurately our samples of $94^{239}, \mathrm{U}^{233}, 93^{237}$.

"10. An investigation to eliminate the possibility that the 40-second period formed in the fission of uranium should be assigned to $U^{239}$ as was originally done by Hahn before the discovery of the fission process."

Norman Hilberry wrote a report covering his visit to Berkeley to discuss and review our research program last week as follows;

"The week was spent going over the work in progress at the University of California, Berkeley, California. The discussions centered in three groupings, that of Seaborg, wahl and their group, that of Kennedy, segrè and Oppenheimer, and that involved with Lawrence and the cyclotron group.

"Seaborg, Wahl, and the chemistry group. - Discussions with this group were concerned with three main subjects. The first of these was the chemical program in progress at Berkeley; the second, the apparatus in use, particularly counting circuits; and finally, the proposal to transfer the work to Chicago.

"I. The program as outlined by seaborg was as follows:

"a. Studies in 93-94.

1. Chemistry-A-I-A priority. 


\section{Physical properties including fission.}

3. The manufacture of large amounts-St. Louis. The efficiency of the st. Louis cyclotron per microampere-hour is less than the efficiency at Berkeley in a ratio of 1 to 3 , but the larger beam at $\mathrm{St}$. Louis makes the total production there practically the same as at Berkeley. Test samples were irradiated at both places and the total activity checked in both cases-also the 2.3-day fraction. No paraffin was used in this case. It is proposed to repeat this experiment and to repeat it with a paraffin geometry also, both at Berkeley and at st. Louis, and also to try the deuteron bombardment of uranium at st. Louis as soon as a target can be prepared. The preparation of larger samples of 94 is quite imperative in order to obtain a better value of the spontaneous fission, the present value being at the limit for the present samples, but not satisfactory. This work is being done by Gofman, Wahl and English.

"b. Properties of $92^{233}$ and $92^{237}$. - This has for its object the preparation of $\mathrm{U}^{23}$, an alpha emitter with a half-life of $10^{5}$ years, by neutron bombardment of thorium 232. The other phase is the neutron bombardment of $92^{238}$ using six or seven Mev neutrons which produces $92^{237}$. Gofman and wahl have been carrying on this work which is practically at its completion.

"c. Properties of $92^{234}$. - For this work an ether extraction is being made of approximately 1,000 lbs. of uranyl nitrate. The $\mathrm{UX}_{1}$ is extracted with a rare earth carrier and allowed to decay. This work is being carried on by I. Perlman, Fontana and Hamilton.

"d. The search in nature for 93 and 94. - This work is being carried on by M. Perlman and Wahl.

"e. Study of the Szilard-Chalmers process for isolating uranium.- This work is being carried out by Breslow.

"II. Seaborg's group operates very largely on the top floor of the Chemistry Building where they have some six or seven rooms set aside for their work. They also have certain other rooms on other floors in the building. The rooms are small but the splitting up into many units is advantageous in that it decreases the possibilities of cross contamination which are always present in radioactive chemistry. The rooms are all equipped with hoods which are in turn equipped with light, gas, water and the special gases required for chemical separation, such as $\mathrm{H}_{2} \mathrm{~S}$, etc. The major portion of the counting is done in one room which is reserved for this purpose. Some counting is carried on in a second room. The counting equipment in use consists of two Geiger-Mueller counter units, two linear amplifier units and one electrometer tube unit. There are in addition available certain portable linear amplifier units equipped with ionization chambers which are portable and usable in any of the rooms. This group also has one laboratory in the old Chemistry Building in which they are carrying on a variety of fission 
measurements and other measurements of this type. This room is also equipped with Geiger-Mueller counting units and with a carefully shielded linear amplifier unit. A variety of other counting units is readily available on loan from Kennedy's group and from the Radiation Laboratory."

Wednesday, April 1, 1942

Isadore Perlman has been working more and more on chemical methods for the extraction of 94 from neutron irradiated uranium in preparation for his impending transfer to the Met Lab in Chicago. He has been studying the concentration of 94 in the mother liquor when UNH is partially crystallized as a potential method for making an initial separation of the bulk of uranium.

Yesterday and today English and wahl attempted to develop a fluoride volatility method for separating 94 from uranium and fission products. They prepared a solution of uranyl nitrate containing 94 and precipitated the uranium and 94 from the solution with hydrogen fluoride to obtain uranous tetrafluoride incorporating 94 fluoride or 94 oxyfluoride. Fluorine was then passed over the mixture of uranium and 94 in their lower fluoride states in an attempt to produce the higher fluorides and for the purpose of determining whether the uranium hexafluoride or the 94 higher fluoride is more volatile. Unfortunately, the experiment was a failure because uranium hexafluoride was not produced-perhaps because of an insufficient supply of fluorine or because the apparatus was not dry.

Dr. Eger V. Murphree, Chief of the OSRD Section S-I Planning Board, visited us today and I described to him the chemical program we have underway here and introduced him to some members of our group. We discussed the problem of the extraction of element 94 from uranium and fission products and in particular the possibility of crystallizing, as UNH, the major portion of the uranium away from the 94, leaving the 94 in solution. We also discussed the possibility of using electrolysis methods for the isolation of the 94 from the mixture that remains after the bulk of the uranium has been removed.

Dr. Glenn E. Sheline, a recent Berkeley Ph.D. in physiology, started to work with my group today. I am asking him to work with Fontana on the isolation of $\mathrm{U}^{234}$ via the extraction of $\mathrm{UX}_{1}$ from large amounts of UNH, thus relieving the efforts of Isadore Perlman who will soon be leaving with me to go to Chicago.

In our informal nuclear seminar this afternoon I gave a survey report on isotope separation. 
During the last several days Gofman has continued his measurements on the slow neutron fission of $\mathrm{U}^{233}$ in Sample $J$ and has now accumulated, including his experiments in February, a total of 453 fission counts in 8.2 hours of counting time, corresponding to 55.2 counts per hour. When the ionization chamber was surrounded with the cadmium shield $10.35 \mathrm{gm} / \mathrm{cm}^{2}$ thickness), 161 counts were obtained in 17.6 hours of counting time, corresponding to 9.1 counts per hour. Therefore, the $\mathrm{U}^{23}$ fission counting rate due to slow (cadmium-absorbable) neutrons amounts to about 46.1 counts per hour. When sample $J$ was replaced by standard sample U-St-6 (200 micrograms of natural uranium containing 1.4 micrograms of $\mathrm{U}^{235}$ ), 330 fissions were obtained in 15.5 hours of counting, amounting to 21.3 counts per hour under the same conditions. When the ionization chamber was surrounded with the cadmium shield, 168 fission counts were obtained in 20.7 hours of counting time, which amounts to 8.1 counts per hour: Thus, the slow neutron fission counting rate of the $\mathrm{U}^{235}$ is 13.2 counts per hour. The ratio of the slow neutron fission counting rate of sample $\mathrm{J}$ to standard sample U-St-6 is therefore

$$
\frac{46.1}{13.2}=3.5
$$

from which the slow neutron fission cross section of $\mathrm{U}^{23}$ relative to that of $\mathrm{U}^{235}$ can be calculated, using the weight of $\mathrm{U}^{233}$ in sample J ( 3.8 micrograms), as

$$
3.5 \times \frac{1.4}{3.8}=1.3
$$

I received a letter from Harold urey dated March 30, 1942 telling me he was glad to receive the copies of the two reports that I mailed to him March 21, 1942. He referred to his letter of March 16, 1942 and said that he wished to clarify what he had in mind when he spoke of our having difficulties in the fluorination process; he said that he had heard from Professor Willard F. Libby that we have had some difficulty in producing $\mathrm{UF}_{6}$ and that he wanted to offer his help. He indicated that Dr. Robert D. Fowler of Johns-Hopkins University and Mr. Priest of his laboratory have much experience in handling fluorides and would be available for consultation. He noted that he has learned from Professor lawrence that I am moving to Chicago to continue my work there.

Friday, April 3,1942

Wahl has made comparative measurements of the alpha activity of Samples B, F, H and $\mathrm{L}$, using our ionization chamber FP-54 electrometer setup, together with comparisons with sample 93-19-1 whose absolute alpha disintegration rate he has determined by measurements using our "inside chamber" $W-3$; he has derived the following weights using the value $2.7 \times 10^{4}$ years as the half-life of $94^{239}$ : Sample B, 0.55 micrograms; Sample F, 1.3 micrograms; Sample H, 1.3 micrograms; and Sample $L$, 2.5 micrograms. Wahl also has measured the relative fission cross 
sections for slow (cadmium-absorbable) neutrons, using the neutrons from our $300 \mathrm{mg}$ Ra-Be neutron source slowed by paraffin; he has compared the slow neutron fission rates of Samples $L, H$ and $F$ with that of U-St-6 (Which contains 200 micrograms of natural uranium and hence 1.4 micrograms of $\mathrm{U}^{235}$ ). He obtained the following results in counts per hour: Sample F, 24; Sample H, 22; Sample L, 45; U-St-6, 15. Using the results for Sample $L$, for example, we can calculate the slow neutron fission cross section of $94^{239}$ relative to that of $\mathrm{U}^{235}$ as

$$
\frac{45}{15} \times \frac{1.4}{2.5}=1.7
$$

This is in excellent agreement with our results of last May. The improved determination of the weight of sample $B$ agrees very well with our estimate of 0.5 micrograms made on May 18, 1941; this new estimate of the weight, then, does not change appreciably our reported value (in the letter to Dr. Lyman J. Briggs of May 29, 1941) that the cross section for fission of $94^{239}$ with slow neutrons is about 1.7 times that of $U^{235}$. On the other hand, a weight of 1.3 micrograms of $94^{239}$ in sample $F$ compared to the weight of 3.5 micrograms estimated on July 13, 1941 indicates that our report (in a letter to Briggs on July 24, 1941) that the cross section of $94^{239}$ with fast neutrons is about 3.4 times that of $\mathrm{U}^{238}$ appreciably underestimated the relative cross section of $94^{239}$.

English completed today his measurements on the spontaneous fission of 50-year 94 commenced on March 4. He has succeeded in operating his counting apparatus in a meaningful way over a period of 120 hours during which no spontaneous fission counts were recorded. The apparatus was tested periodically during this interval by replacing the 50-year 94 sample with a uranium sample and demonstrating that the fissions induced in this uranium by a neutron source were being recorded with the proper efficiency. It was shown, by a consideration of the geometry of the ionization chamber and by measuring the variation with amplifier gain of the neutron-induced uranium fission counting rate, that the overall efficiency of the apparatus for recording fissions amounted to about $55 \%$. Hence, for a 0.005 microgram sample the time between fission counts should be, on the average, about 17 hours, for a spontaneous fission rate corresponding to a "half-life" of $10^{10}$ years. Since we observed zero counts in 120 hours, it seems quite certain that the "half-life" for the spontaneous fission of the 50-year 94 exceeds $10^{10}$ years.

Prestwood, Auerbach and Bonner, joined by Sheline who has just come aboard, performed the first ether extraction on Batch VI. They spent yesterday and the day before getting the batch in shape for ether extraction by melting it and allowing it to recrystallize during intense stirring, a procedure that retains the $\mathrm{UX}_{1}$ and puts the UNH in shape for ether extraction as a substitute for the tedious grinding procedure. They finished the extraction of this batch, in which the $\mathrm{UX}_{1}$ is fully grown, in 6 hours a tribute to their experience.

Breslow and Hamaker have completed two successful experiments eliminating the use of pyridine for the concentraction of $\mathrm{U}^{237}$ by the 
Szilard-Chalmers method. In these experiments $10 \mathrm{gm}$ of salicylaldehydeo-phenylenediamine uranyl together with $30 \mathrm{mg}$ of uranyl acetate to act as carrier for the $\mathrm{U}^{237}$ were bombarded with neutrons from the 60 -inch cyclotron (bombardments no. $\mathrm{U}, \mathrm{n}-20$ and no. $\mathrm{U}, \mathrm{n}-2 \mathrm{l}$ ). The organic compound was then dissolved in $100 \mathrm{cc}$ of aniline, and the solution was extracted with three small portions of saturated sodium sulfate solution in water. This sodium sulfate solution contained the carrier uranium and $\mathrm{U}^{237}$ and the fission products, from which the uranium was precipitated by the addition of ammonium hydroxide. The precipitate was dissolved in the minimum amount of nitric acid and the fission products were removed by the same ether extraction method used in the experiments of March 11 . In these two such experiments the yield of $\mathrm{U}^{237}$, present in about $10 \mathrm{mg}$ of uranium, amounted to some 10 microcuries per 100 microampere-hours of deuterons on beryllium, again indicating that a szilard-Chalmers concentration has taken place.

Saturday, April 4, 1942

Fontana and Bonner completed the extraction of the UX 1 fraction from Batch $V$, starting with a volume of 20 liters, using the usual procedure of precipitation of rare earth fluoride, concentration by precipitation of the iodate, precipitation of the fluoride, and then dissolution in acid, to set aside for decay of the $\mathrm{UX}_{1}$ to daughter $\mathrm{U}^{234}$.

Sunday, April 5, 1942

Yesterday and today Gofman made measurements of the slow neutron fission rate due to the 0.8 microgram of $\mathrm{U}^{23}$ in sample $\mathrm{Th}+\mathrm{n}-\mathrm{I}-\mathrm{Pa}-\mathrm{III}$. Again, he used the $300 \mathrm{mg}$ Ra-Be source and placed the sample on one electrode of an ionization chamber imbedded in paraffin and made measurements with and without a cadmium shield. His counting rate of 11.2 counts per hour ( 21.8 hours of counting time) without the cadmium shield and 1.9 counts per hour ( 11.5 hours of counting time) with the cadmium shield indicates 9.3 counts per hour due to slow neutron fission. This counting rate of about one-fifth of that obtained with Sample $J$, with this sample which has about one-fifth as much $U^{233}$, again leads to the result that the slow neutron fission cross section of $\mathrm{U}^{23}{ }^{3}$ is about 1.3 times that of $U^{235}$.

Monday, April 6, 1942

In order that we might have a sample of $94^{239}$ of maximum intensity to measure its spontaneous fission rate and so forth, Wahl is today starting to combine Samples $\mathrm{F}$ ( 1.3 micrograms), H ( 1.3 micrograms) and I. ( .5 micrograms). The combined sample will be labelled Sample $M$. He plans to further separate these samples from fission products by 
subjecting them to oxidation-reduction cycles using $\mathrm{S}_{2} \mathrm{O}_{8}^{-2}$ plus Ag ion as oxidizing agent, $\mathrm{SO}_{2}$ as reducing agent, and hopes to use a final small amount of $\mathrm{LaF}_{3}$ as carrier in order to obtain a thin sample.

The second ether extraction on Batch VI was completed with some trouble because the Mallinckrodt UNH had some impurities. They handed over a solution of about 2 pounds of UNH in $1800 \mathrm{CC}$ to Fontana and Sheline for extraction of the UX. This contained only about 4 millicuries, some $25 \%$ of the expected yield, indicating that there is some $\mathrm{UX}_{1}$ in the crud which they will leach with acid and also give to. Fontana and sheline.

I received from Leo Szilard a letter dated April 3, 1942 saying that rumors have reached him that a large sample of uranium oxide was purified in my laboratory by the method of ether extraction and that considerable amounts of impurities were found. Since this uranium oxide might be of the same quality as the 60 tons that have been ordered by Murphree for the Metallurgical Project, he would like to have full information on the amounts of impurities found and on the origin of the sample so that he can identify it in his discussions with Eldorado Gold Mines Ltd.

The large Lawrence contract (temporary no. SSRC-1) has been assigned the Contract No. OEMsr-324, Symbol no. S-26.

Tuesday, April 7, 1942

Today Gofman measured the range of the alpha particles from $U^{23}$ by comparison of the absorption of these in aluminum foils with the absorption of $\mathrm{Po}^{210}$ alpha particles (sample Po-st-6) measured under the same conditions. He used our low geometry ionization chamber-linear amplifier-magnetic field setup $(w-1)$, with the magnetic field turned off. He finds the range of $\mathrm{Po}^{210}$ to be longer than that of $\mathrm{U}^{233}$ by $1.1 \mathrm{mg}$ per sq. $\mathrm{cm}$ of Al. Differences in range between the alpha particles can be converted to differences in air range at $760 \mathrm{~mm}$ and $15^{\circ} \mathrm{C}$. using the Livingston and Bethe (Livingston and Bethe, Rev. Mod. Phys. 9, 276 [1937]) conversion factor of $1.52 \mathrm{mg}$ per sq. $\mathrm{cm} \mathrm{Al}$ per $\mathrm{cm}$ of air. Thus the range in air of $\mathrm{U}^{233}$ alpha particles is $0.72 \mathrm{~cm}$ less than that of $\mathrm{PO}^{210}$ (whose range is $3.92 \mathrm{~cm}$ ), or $3.2 \mathrm{~cm}$ of air at standard conditions $\left(760 \mathrm{~mm} \mathrm{Hg}\right.$ and $\left.15^{\circ} \mathrm{C}.\right)$. Taking into account his results of February $18-19$ (a range of $3.0 \mathrm{~cm}$ ), the best value for the range is $3.1 \pm 0.2 \mathrm{~cm}$, corresponding to an energy of $4.8 \mathrm{Mev}$. These data for $\mathrm{U}^{23}$ are the first obtained for an alpha emitter of the missing $(4 n+1)$ radioactive series. It is interesting to note that this point falls near the Geiger-Nuttal curve for the uranium $(4 n+2)$ series.

Morris Perlman has started to work on the extraction of any 94 or 93 that might be present in a sample of carnotite ore (a potassiumuranium vanadate from Colorado, which we have acquired). I have asked Norman Bonner to work with him on this. Perlman and Bonner started today to dissolve the carnotite ore. 
Using material provided by Morris Perlman, I began to write the report "Search for Elements 94 and 93 in Nature; Presence of $94^{239}$ in Pitchblende" to be issued under the authorship of Perlman and me. It is planned to describe the results of our search for 94 and 93 in the $400 \mathrm{gm}$ sample of pitchblende ore concentrate obtained from the Great Bear Lake region of Canada.

Wednesday, Apri1 8, 1942

Since he undertook his experiments on the electrolytic deposition of 94 on February 15, 1942, interspersed with his work on the $\mathrm{U}^{23^{3}}$ problem, Gofman has tested a wide range of conditions for the electrolytic deposition. He has made experiments with 94 in the oxidized state using a number of oxidizing agents such as periodate ion, peroxydisulfate ion plus silver ion, and has tested the conditions of acidity and the use of buffer solutions. Today, using peroxydisulfate ion as an oxidizing agent, a concentration of acetate ion of about $1 \mathrm{M}$. and seven hours of electrolysis, he obtained a good adherent plate on copper with a $70 \%$ yield of 94 . His yields of 94 from solutions in which it is present in the reduced state have been negligible under similar conditions. This might form the basis for a separation procedure beginning with a solution in which the bulk of the uranium has been removed and in which the 94 is first present in its reduced form; from this solution fission products might be deposited electrolytically and then, after oxidizing the 94 , the 94 might be deposited in a relatively pure form.

Working in room 234, Old Chemistry Building, Gofman is beginning his measurements on the fast neutron fission cross section of $\mathrm{U}^{23}$ using our new $1 \mathrm{gm}$ radium-beryllium source acquired from the Canadian Radium and Uranium Company in New York. We now have available a sample prepared by Kennedy from the output of the uranium isotope separation using the 37-inch cyclotron magnet. This sample contains 1.3 micrograms of $\mathrm{U}^{235}$ and 5.2 micrograms of $\mathrm{U}^{238}$ (and $1.4 \times 10^{-3}$ microgram of $\mathrm{U}^{234}$ ); thus, it is possible by also using a sample of natural uranium to make comparative measurements of the fast neutron cross section of $U^{23}$ in comparison with those of $\mathrm{U}^{235}, \mathrm{U}^{238}$, as well as $94^{239}$ (using our sample $\mathrm{B})$ and $\mathrm{Pa}^{231}$ (being isolated by stoughton). Gofman has built a fissiondetecting ionization chamber, by the use of cadmium plate and boron carbide, with an optimal arrangement for measuring the cross sections due to fast neutrons.

Isadore Perlman completed today the experiments I have asked him to perform to crystallize uranium, as UNH, away from element 94 . His results indicate that after the removal of $90 \%$ of the original uranium by crystallization as UNH, about $60-65 \%$ of the 94 remains in solution.

Fontana and Sheline finished their extraction of UX, from Batch VI. They used the usual procedure, starting with 10 liters of volume, added $0.5 \mathrm{gm}$ of $\mathrm{La}^{++}$and $20 \mathrm{mg}$ of $\mathrm{Ce}^{++++}$as carrier, then after separating the $\mathrm{Ce}^{+++}$containing the $\mathrm{UX}_{1}$, set the solution aside for decay. 
I received a letter from Frank Spedding dated April 6, 1942 informing me that arrangements for our move to Chicago are well underway, and indicating that he is planning a chemical conference at the Metallurgical Laboratory for the dates of April 20-24, 1942.

I completed putting together the report by Morris L. Perlman and me, "Search for Elements 94 and 93 in Nature; Presence of $94^{239}$ in Pitchblende." In this report we describe in detail the process for dissolving $400 \mathrm{gm}$ of pitchblende concentrate (from the Great Bear lake region of Canada) and the isolation from this by our oxidation-reduction procedure using rare earth fluoride as carrier, a final fraction which should contain any 94 or 93 that might be present. We indicate that this final sample shows no detectable beta activity but does show an alpha counting rate when placed on one electrode of an ionization chamber in which the calibrated counting efficiency is about $45 \%$. On the assumption that this alpha counting rate is due to $94^{239}$, we calculate a concentration of about one part in $10^{14}$ of $94^{239}$ in the original pitchblende concentrate and speculate that this might be due to the absorption of spontaneous fission neutrons in the $U^{238}$ present in the pitchblende.

Thursday, April 9, 1942

Gofman has run into trouble with his expeximents on measuring fast neutron cross sections using our new I gm Ra-Be neutron source because this source has sprung a leak resulting in radon contamination of the ionization chamber.

He has obtained the final $30 \mathrm{CC}$ solution of the combined $94^{239}$ from the residues of bombardments no. 93-18 and $U, n-15$ which he labeled sample $\mathrm{U}+\mathrm{n}-15-18-\mathrm{F}$. His assay showed that this solution contains a total of $94^{239}$ alpha particles corresponding to 426,000 disintegrations per minute.

I responded to Leo Szilard's letter of April 3, 1942 giving him information on the work we have been doing to extract large amounts of uranium-free $U_{1}$ from large quantities of UNH. I identified the five large batches of UNH that we have received (two from General Chemical Company, two from Eldorado Gold Mines Ltd., and one from S. W. Shattuck Chemical Company) and compared the amounts of impurities that we found in each.

I wrote to Spedding at the Metallurgical Laboratory, Chicago, to send him an equipment list and list of books that might be acquired for my group's use when we arrive in Chicago. I also indicated that we are preparing to send some of the more vital. equjpment from Berkeley and this should arrive sometime next week. I indicated that Isadore perlman and I will arrive in Chicago and be ready to start work on April 20th and that Spofford English will come a few days later (probably April 24). I told him that English will help us set up our Geiger counter and linear amplifier counting circuits. He is one of my graduate students who has not yet completed his Ph.D. degree work and may return to Berkeley in 
the fall. I also indicated that I have been talking to two or three B.S. chemists who are graduating from Berkeley next month, with the thought of bringing them to Chicago. I indicated that I will bring with us to Chicago plenty of stock solution of tracer 50-year 94 so that we will be well equipped to get started immediately.

Friday, Apri1 10, 1942

I wrote to Dr. Eger V. Murphree to tell him about Perlman's recent results of the partial separation of element 94 . from uranium by crystallizing UNH and the results of Gofman on the isolation of 94 by electrolysis. I suggested that a total separation process might involve (1) the separation of the major portion of uranium as UNH and (2) the final isolation of the 94 by the electrolytic method using this oxidizing and reducing process. I told him that I am transferring to Chicago with a few of my people so this might increase the possibility of my visiting him in New York.

Using material provided by Wahl, I began to put together the report "Properties of $93^{237}$ " to be authored by wahl and me. This report will describe our discovery of $93^{237}$ and its radioactive and fission properties.

Saturday, April 11, 1942

Friedlander and Stoughton made a bombardment of uranium with deuterons (10,000 microampere-hours) in order to produce a stock of 50-year 94 tracer to meet the increasing demands of our group (bombardment no. U,D-3). Stoughton began the chemical isolation procedure on half the sample by precipitating LaF 3 from the dissolved solution, dissolving it in hot concentrated $\mathrm{H}_{2} \mathrm{SO}_{4}$ and then reprecipitating it. The plan is to isolate the 94 fraction using the selective oxidation-reduction procedure where 93 and 94 are oxidized to fluoride soluble form by $\mathrm{S}_{2} \mathrm{O}_{8}{ }^{-2}$ plus silver ion, while only 93 is oxidized to fluoride soluble form with a cold $\mathrm{BrO}_{3}^{-}$solution. In order to learn how to do it, Isadore Perlman is isolating the 50-year 94 from the other half of the sample using the same chemical procedure.

I completed writing the report "Properties of $93^{237 "}$ by wahl and me. The report includes a description of the method of production of the $93^{237}$ and reports its half-life for alpha decay to be about $3 \times 10^{6}$ years and the upper limit for the cross section for its fission with slow neutrons to be $1 \%$ of that of $\mathrm{U}^{235}$.

Using material provided by English, I began to write the report "Search for Spontaneous Fission in 50-Year 94" by English and me. 
I completed writing the report "Search for Spontaneous Fission in 50-Year 94" by English and me. This described our method of production and isolation of a 0.005 microgram sample of 50-year 94 and our experiments to test for its spontaneous fission by placing it on one electrode of an ionization chamber connected to a linear amplifier and recording system. The absence of any fission counts during 120 hours of counting indicates that the half-life for spontaneous fission probably exceeds $1.0^{10}$ years.

I wrote an abstract of our report "Properties of $U^{23}$ " authored by Gofman, Stoughton and me. The exigencies of my schedule in getting ready for my move to Chicago prevents me from preparing the whole report at this time and so. I will get to this soon after my arrival at the Metallurgical Laboratory. We report in the abstract that we have prepared by neutron bombardment of $\mathrm{Th}^{232}$ a sample of $\mathrm{U}^{233}$ weighing 3.8 micrograms and with this have demonstrated that this isotope undergoes fission with slow neutrons with a cross section about 1.25 times that of $U^{23}$, has a spontaneous fission "half-life" greater than $10^{14}$ years, and decays by the emission of alpha particles of range $3.1 \pm 0.2 \mathrm{~cm}$, with a half-life of $1.2 \times 10^{5}$ years.

Monday, April 13, 1942

Today there was a briefing by members from the Army Intelligence Division concerning the importance of secrecy on our project. It took place at 2:00 p.m. in room 213 Leconte Hall and all members of my group, including myself, and all members of the project were required to attend.

Fontana has completed the extraction of the $\mathrm{UX}_{1}$ from the Denver Brown Mud and set this aside to decay to the daughter $\mathrm{U}^{234}$.

Using material provided by Gofman, I wrote a short report "Slow Neutron Fission of $\mathrm{U}^{234}$ " with today's date. This report, under the authorship of Gofman and me, reports measurements on our 0.1 microgram sample of $\mathrm{U}^{234}$, isolated via extraction of $\mathrm{UX}_{1}$ from UNH, with the result that the slow neutron fission cross section is of the order of one-half that of $\mathrm{U}^{235}$ (an upper limit).

David S. Breslow and John Hamaker gave me their report "SzilardChalmers Process for Uranium" in which they describe their work on the concentraction of the 6.8-day $\mathrm{U}^{237}$ produced by the bombardment of uranium with the fast neutrons from the Berkeley 60-inch cyclotron. They report that the use of salicylaldehyde-o-phenylenediamine uranyl results in an appreciable concentration of $\mathrm{U}^{237}$ by the szilard-Chalmers effect, whereas a procedure with inorganic uranium compounds to take advantage of the two oxidation states of uranium, uranyl $(+6)$ and uranous $(+4)$, was unsuccessful. 
I wrote to H. T. Wensel, Technical Aide, Section S-1, NDRC in Washington, sending him a copy of the report "Search for Elements 94 and 93 in Nature; Presence of $94^{239}$ in Pitchblende" authored by Morris Perlman and me. I indicated that this should supplement, as a final report of work done on NDCrc-197, the preliminary report authored by wahl and me sent to him on February 16, 1942. I also sent a copy of this report to Harold urey at Columbia University.

Also today Isadore Perlman and Beppino Fontana handed me their report "Preparation of $\mathrm{U}^{234}$ Through UXl. In this they give an overall description of the method that their group has developed for the isolation of 24.1-day UX $\mathrm{UX}_{1}$ from large quantities of UNH and the subsequent isolation of the $U^{234}$ from the $\mathrm{UX}_{1}$. They include a description of a method for electroplating the final sample of $\mathrm{U}^{234}$ onto a platinum foil.

NOTE: The paper "Search for Elements 94 and 93 in Nature; Presence of $94^{239}$ in Pitchblende" was issued by the s-1 Committee in washington under the report number A-146 dated April 13, 1942 with authors G. T. Seaborg and M. I. Perlman. After the war it was published in J. Am. Chem. Soc. 70, 1571 (1948).

"Preparation of $\mathrm{U}^{234}$ through $\mathrm{UX}_{1}$ " was issued by the $\mathrm{S}-1$ Committee as report number A-172 dated April 13, 1942 with authors I. Periman and B. J. Fontana.

"Slow Neutron Fission of $U^{234 " ~ b y ~ J . ~ W . ~ G o f m a n ~ a n d ~ G . ~ T . ~ S e a b o r g ~}$ was issued by the S-1 Committee as report number A-173 dated April 13, 1942 .

"Szilard-Chalmers Process for Uranium" by D. S. Breslow and J. W. Hamaker was issued as report number $A-174$ by the $S-1$ Committee dated April 13, 1942.

Tuesday, April 14, 1942

Wahl has nearly completed his isolation of a combined $94^{239}$ sample, Sample M (from Samples F, H and I started on April 6), with indications from alpha measurements on aliquots of his solutions that he may recover a total of close to 5 micrograms.

Stoughton completed the isolation of 50-year 94 from bombardment no. U,D-3 by the chemical procedure he started last Saturday. His assay of the final $50 \mathrm{cc}$, which will serve as a stock solution for our group, indicates the presence of an amount of 50-year 94 corresponding to $9,280,000$ disintegrations per minute, corresponding to a yield of about $3 \times 10^{-5}$ microgram per microampere-hour of deuterons. This $50 \mathrm{cc}$ of stock solution was dispersed as follows: $8 \mathrm{cc}$ each to Gofman, Stoughton, Friedlander and wahl to serve as tracer for their investigations of the 
chemistry of element 94 ; and $18 \mathrm{cc}$ to me to transfer to Chicago to serve as tracer for my program on 94 chemistry that will get underway there later this month. Isadore Perlman also completed the isolation of 50year 94 from his half of the sample and obtained a yield of about $2 \times 10^{-5}$ micrograms per microampere-hour of deuterons.

I wrote to Professor Arthur H. Compton enclosing copies of our reports "Properties of $\mathrm{U}^{233 "}$ (an abstract) by Gofman and me, "Properties of $93^{237 "}$ by Wahl and me (both dated April 13, 1942), and "Production and Properties of 50-Year Element 94" dated March 20, 1942. I also indicated that $\mathrm{Dr}$. Alexander Langsdorf has bombarded uranium with deuterons for us at St. Louis so that we can compare the production of 50-year 94 with that produced in Berkeley; we have found that the yield at St. Louis is of the order of $1 / 10$ as much as that at Berkeley.

I also wrote to Dr. Briggs in Washington enclosing these three reports and indicating that this represents the work done under my contract OEMsr-206. I also informed Dr. Briggs that I am transferring a portion of my men and work to Chicago.

NO'lE: The paper "Properties of $\mathrm{U}^{23^{3}}$ (abstract) was issued immediately by the S-l Committee in Washington as report number A-153 dated April 13, 1942 with authors G. T. Seaborg, J. W. Gofman and R. W. Stoughton. After the war it was published under the changed title "Nuclear Properties of $\mathrm{U}^{23^{3}}$ : A New Fissionable Isotope of Uranium" by G. T. Seaborg, J. W. Gofman and R. W. Stoughton in Phys. Rev. 71, 378 (1947).

"Properties of $93^{237 "}$ by A. C. Wahl and G. T. Seaborg was issued by the S-1 Committee as report number A-151 dated April 13, 1942. It was published after the war under the changed title "Nuclear Properties of $93^{237}$ " by A. C. Wahl and G. T. Seaborg in Phys. Rev. 73, 940 (1948).

Wednesday, April 15, 1942

In view of my impending departure for Chicago, I have asked Rene Prestwood, Norman Bonner and Charles Auerbach to prepare reports covering the work they have done with me this semester as part of chemistry course $180 \mathrm{H}$. I asked prestwood to emphasize the ether extraction process for the concentration of $\mathrm{UX}_{1}$, Auerbach to emphasize the process for the recovery of the uranyl nitrate after the ether extraction process, and Bonner the process for separation of UX ${ }_{1}$ from the ether aqueous extract through precipitation with rare earth fluoride. When they handed me these reports today, I remarked in jest to Prestwood and Auerbach that these reports looked like they deserve "good strong C's" for their grades in the chemistry $180 \mathrm{H}$ course. Prestwood and Auerbach reacted with some consternation, but I believe they really know I am kidding. Bonner has not completed his report, which he will send to me at Chicago within a week or two. 
Prestwood's report "Concentration of $\mathrm{UX}_{1}$ by Extraction of Uranyl Nitrate with Diethyl Ether" describes the procedures that he has helped develop, in conjunction with Isadore Perlman, Beppino Fontana, Auerbach and Bonner, for the extraction of $\mathrm{UX}_{1}$ from large amounts of uNH, using the method of partition between ether and water, and for the recrystallization of the UNH. Auerbach's report "The Recovery of Uranyl Nitrate After the UXI Extraction with Diethyl Ether" describes the work that he has been doing this semester along with the same group on the recovery of uranyl nitrate to put it back into crystalline form, suitable for further ether extraction, after extraction with ether.

Isadore Perlman has completed a determination of the yield of 50year 94 produced by the bombardment of uranium with the $12 \mathrm{Mev}$ deuterons in the 45-inch cyclotron at Washington University in St. Louis (bombardment no. U,D-4). He finds a yield of about $3 \times 10^{-6}$ microgram per microampere-hour of deuterons. This is to be compared with an average yield (3 determinations) of about $2.5 \times 10^{-5}$ microgram per microamperehour from deuterons in the 60-inch cyclotron here in Berkeley whose energy is about $14 \mathrm{Mev}$ when allowance is made for absorption in the aluminum window in front of the uranium target.

In our informal nuclear seminar this afternoon Perlman gave a survey on the applications of tracers to biological research.

Thursday, April 16, 1942

Stoughton completed the isolation of $\mathrm{Pa}^{231}$ from the Great Bear Lake pitchblende, a long, complicated chemical procedure that he started on March 13 and upon which he has been working since that date, interspersing his efforts with those on the $U^{23}$ problem. His final electrolytic deposition was performed between 1:40 a.m. and 9:50 a.m. this morning. We are designating this as Sample $P$. (Although the logical sequence of letter designations would suggest that this be designated Sample $N$, we have not used this in order to avoid confusion with the sound of Sample $M$; we have also avoided the designation Sample $O$ in order to avoid confusion with the number zero.) Gofman determined the intensity of the $\mathrm{Pa}^{231}$ alpha particles from this sample by counting them with ionization chamber $\mathrm{W}-2$ in a position of low geometry through a calibrated screen to reduce the counting rate to manageable proportions. He finds a counting rate which corresponds to 112,000 alpha particles per minute. Using a half-life of 32,000 years for $\mathrm{Pa}^{231}$, we can calculate that its alpha emission rate is 108,000 per minute per microgram. Hence our Sample P contains

$$
\frac{112}{108}=1.04 \text { micrograms } \mathrm{Pa}^{231} .
$$


The leak of the $1 \mathrm{gm}$ Ra-Be neutron source has been temporarily fixed and Gofman and Friedlander are continuing with the measurement of the comparative fast neutron fission cross sections of $\mathrm{U}^{23}, \mathrm{U}^{235}, \mathrm{U}^{238}, 94^{239}$ and now $\mathrm{Pa}^{231}$.

Prestwood and Auerbach have finished the recovery of all the UNH from Batches I through VI which have a combined weight of about 900 pounds. Out of this will come 200 pounds for wahl to use for a huge bombardment with neutrons at the 60 -inch cyclotron to begin immediately for the production of a large quantity of $94^{239}$. Also, I will request that 300 pounds be allocated for a similar bombardment with the neutrons at the St. Louis cyclotron to produce a supply of $94^{239}$ for the use of my group at the Met Lab. The remaining 400 pounds can be used for further extraction of $\mathrm{UX}_{1}$ so that this source, together with the samples that are already growing $U^{234}$, including the Denver Brown Mud source, should make it possible to prepare larger samples of $U^{234}$ in the future.

Today is the day that I am scheduled to leave with Isadore Perlman to take up my position at the Metallurgical Laboratory at the University of Chicago. In preparation for this, we are shipping to chicago a supply of 50-year 94 tracer and some counters and counting circuits and other critical equipment in order to be in a position to get a good start.

Perlman will accompany me so as to be at the Met Lab from the very beginning, and English will join us there within a week or two. With that start we will try to build up our group by hiring new people.

The remainder of my group will stay at Berkeley to carry on the research program and I hope to interest Dean Wendell $\mathrm{M}$. Latimer to take an interest in, and serve as a leader for, this continued program. Wahl will continue to work on the chemistry of 94 with emphasis on the development of the chemical process for the separation of the product $94^{239}$ from uranium and fission products formed in the nuclear chain reaction. Gofman, working with Friedlander, will finish the measurements on the relative fast neutron fission cross sections of $\mathrm{U}^{233}, \mathrm{U}^{235}, \mathrm{U}^{238}, 94^{239}$ and $\mathrm{Pa}^{231}$. He will also work on other aspects of induced radioactivity in thorium, such as the products of deuteron bombardment. He will, however, put an increasing amount of his time on the development of chemical procedures for the isolation of $94^{239}$ from uranium and fission products.

Stoughton will continue his work on $U^{233}$ and the development of methods for its separation from thorium and fission products.

Morris Perlman and Bonner will work on the search for 94 and 93 in nature and may be joined by Clifford $\mathrm{s}$. Garner should he decide to join our group; they will work on the carnotite ore from colorado and the fergusonite and hatchettolite ores from the Fansteel Metallurgical Corporation. Fontana, sheline and Prestwood will continue on the isolation of larger samples of $\mathrm{U}^{234}$, after which they will probably transfer to the general problem of 94 chemistry. Hamaker and Breslow will continue, at least for a while, on the development of the szilard-chalmers method for the isolation of $\mathrm{U}^{237}$ from neutron-bombarded uranium. It will 
be my hope to obtain permission for Friedlander to assume an official position with our group because with the new security provisions it is becoming difficult, if not impossible, for him to continue much longer to work on the various nuclear and chemical aspects of the properties of element 94 as he has been doing unofficially up until now.

In the late afternoon Isadore Perlman and I boarded the streamliner "City of San Francisco" for our move to Chicago.

This, then, terminates my account of the early Berkeley work. It will be continued as part of my description of the continuing work at the Metallurgical Laboratory at Chicago. 
A $P$ P E N D I X

List of present addresses of persons mentioned in text.

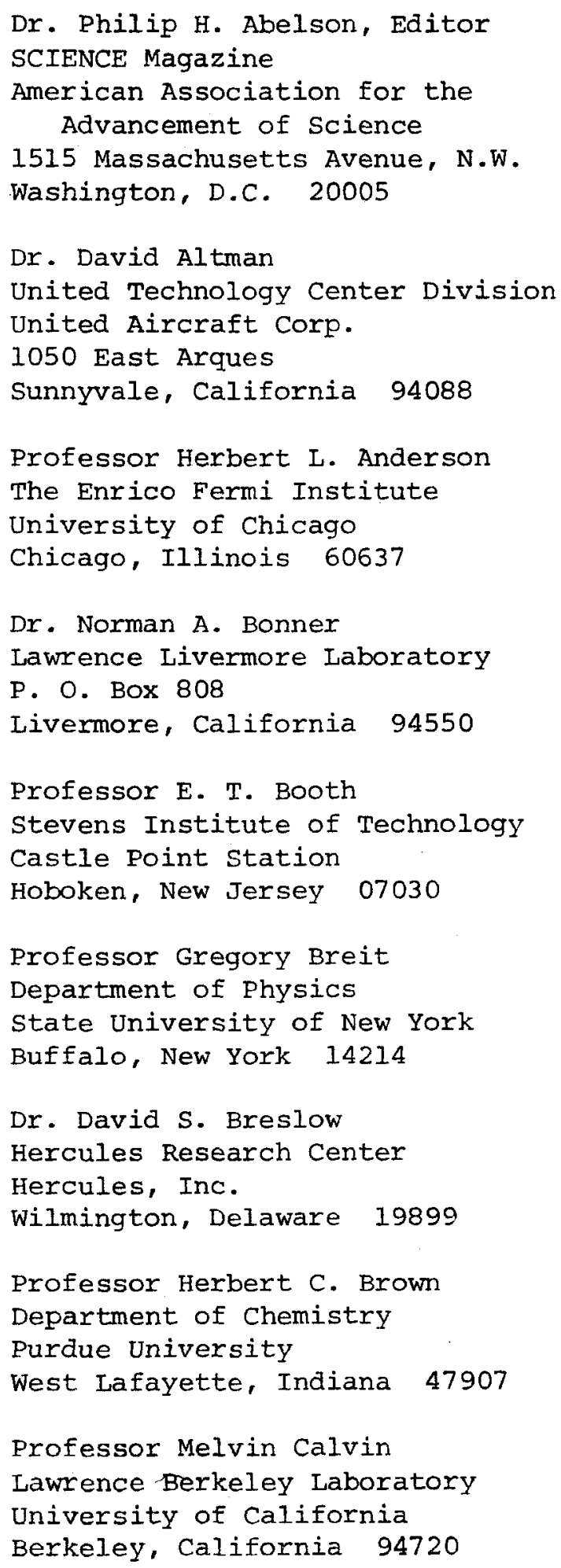

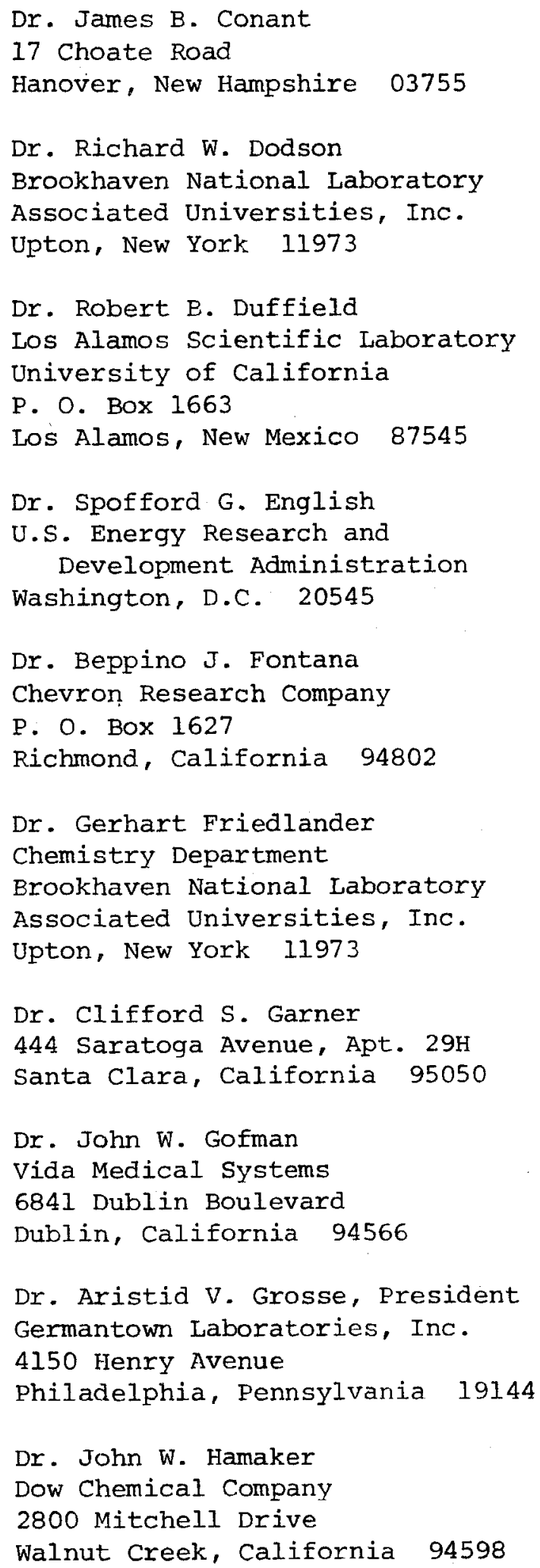


Professor A. Carl Helmholz Department of Physics University of California Berkeley, California 94720

Professor Norman Hilberry Department of Nuclear Engineering College of Engineering

Arizona University

Tucson, Arizona 85721

Professor Joel H. Hildebrand 500 Coventry Road Berkeley, California 94707

Professor Martin D. Kamen Department of Chemistry University of Southern California University Fark

Los Angeles, California 90007

Dr. Joseph J. Katz

Chemistry Division

Argonne National Laboratory 9700 South Cass Avenue

Argonne, Illinois 60439

Professor Donald w. Kerst

Department of Physics

University of Wisconsin

Madison, Wisconsin 53706

Dr. Alexander Langsdorf, Jr. Argonne National Laboratory 9700 South Cass Avenue Argonne, Illinois 60439

Dr. Frederick J. Leitz, Jx. Hanford Engineering Development Laboratory

P. O. Box 1970

Richland, Washington 99352

Professor Willard F. Libby Institute of Geophysics and Planetary Physics

University of California

405 Hilgard Avenue

Ios Angeles, California 90024
Professor Franklin A. Long

Cornell university

Ithaca, New York 14850

Dr. Winston M. Manning

5524 Carpenter

Downers Grove, Illinois 60515

Professor Edwin M. MCMillan

Lawrence Berkeley Laboratory

University of California

Berkeley, California 94720

Dr. Paul G. Nahin (Nahinsky)

Union Oil Company

Research Department

Brea, California 92621

Professor Thomas H. Norris

Department of Chemistry

Oregon state university

corvailis, oregon 97331

Professor Edwin F. Orlemann

Department of Chemistry

University of California

Berkeley, California 94720

Professor Isadore Perlman

Institute of Archaeology

The Hebrew University of Jerusalem

Jerusalem, Israel

Dr. Morris L. Perlman

Brookhaven National Laboratory

Associated Universities, Inc.

Upton, New York 11973

Mr. Alexander Pregel

21 bis Rue de Paradis

75010 Paris, France

Dr. Rene J. Prestwood

Los Alamos Scientific Laboratory

P. O. Box 1663

Los Alamos, New Mexico 87544 


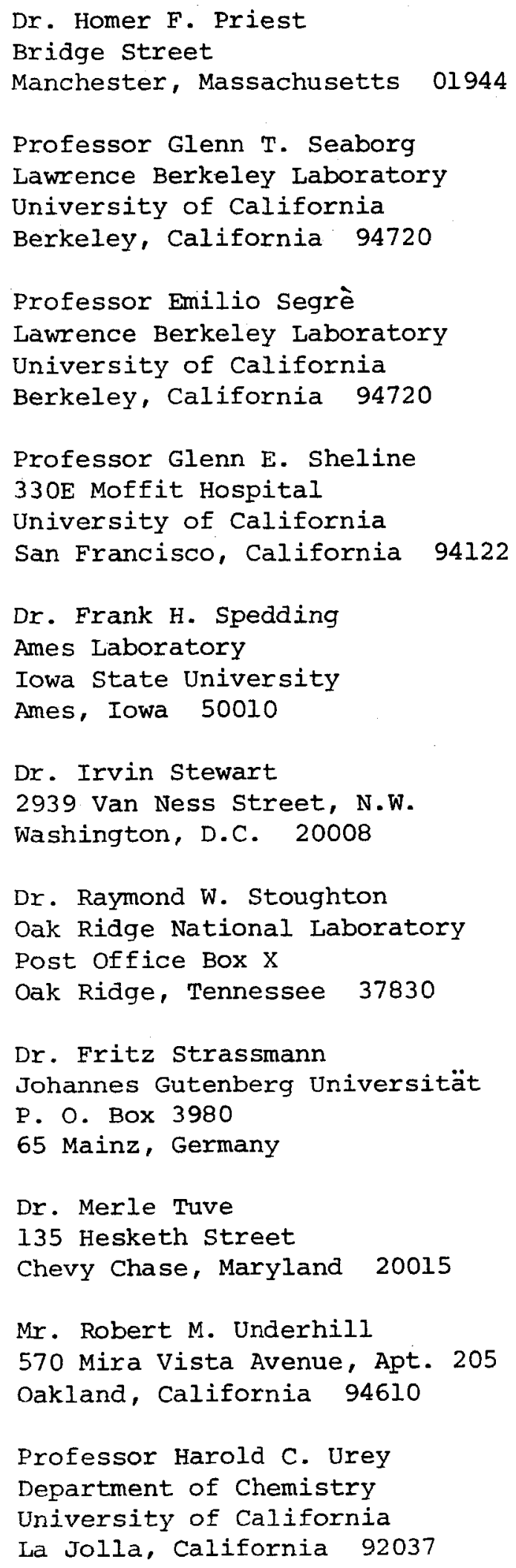

Professor John H. Van Vleck Department of Physics Harvard University Cambridge, Massachusetts 02138

Professor Arthur C. Wahl Department of Chemistry Washington University st. Louis, Missouri 63130

Professor John A. Wheeler Department of Physics Princeton University Princeton, New Jersey 08540

Professor Don M. Yost 1270 Cardova Street, Apt. 9 Pasadena, California 91106 
Abelson, Philip H., 1,14,17, 18,19, $20,30,35,40,53$

Adams, Gayle E., 84,119

Agruss, Bernard, 57

Altman, David, 60,117

Anderson, Herbert L., 134

Archer, R. S., 104

Auerbach, Charles E., 89,94,108, $119,132,138,146-47,148$

Balke, Clarence w., 54,65,72

Barter, Cyril, 60,74,103

Benbow, B. L., 104

Bethe, Hans A., 94,140

Bonnex, Norman A., 55,60,83-84,85, $86,87,90,91,93,94,95,97,103$, $108,113,118,119,121,132,138$, $139,140,146-47,148$

Booth, Eugene T., 6,21

Breit, Gregory, 20,31,44,53,59,60, $61,64,73,76,81,104,110$

Breslow, David S., 91,95,96,102, $114,120,128,135,138,144-45,148$

Briggs, Lyman J., $17,18,19,20,30$, $31,35,40,43,44,45,47,49,52,53$, $60,64,66,70,73,74,78,80,83,95$, $107,127,138,146$

Brown, Herbert C., 104

Bush, Vannevar, $42,43,45,52,53,74$, 77,95

Calvin, Melvin, 60,83

Carroll, H., 117

Compton, Arthur C., 42,43,74,78, $81,83,104,110,114,115,116,124$, $127,128,129,131,132-33,146$

Conant, James B., 74,78,83,95,99

Coolidge, William D., 42

Crowell, William R., 33

Dodson, Richard W., 10

Duffield, Robert B., ii,60,83-84, 102

Dunning, John R., 21

English, Spofford G., ii, $2,14,47$, $50,60,67,80,83,88,108,115,116$, $117,128,132,135,136,138,142$, $143,144,148$
Fermi, Enrico, 9,13,14,17,18,19; $20,21,104,134$

Fontana, Beppino J., ii, $84,85,86$, $87,90,91,93,94,95,97,108,112$, $114,119,121,123,128,130,132$, $135,136,139,140,141,144-45,147$, 148

Fowler, Robert D., 94,137

French, Carl, 54,60

Friedlander, Gerhart, ii, 1,3,9,60, $78,80,84,117,128,132,143,145$. $148-49$

Garner, Clifford S., 105,148

Giauque, William F., 106

Gibson, George E., 60

Gofman, John W., $i-i i, 2,3,4,5,6$, $8,13,14,16,17,21,35,37,38,47$, $48,50,54,56,59,60,65,66,69,70$, $71,74,77,78,79,80,83-84,85,86$, $89,90-91,96,97,98,99,100,101$, $102,103,105,107,108,111,112$, $113,115,116,123,126,127,128$, $130,131,132,135,137,139,140$, $141,142,143,144,145,146,148$

Grahame, David C. , 65,94 Grosse, Aristid v., 6,21,57

Hagiwara, T., 60

Hahn, Otto, 2,134

Hamaker, John W., ii, 33,43,54,60, $74,83-84,91-92,95,120,121,124$, $138,144-45,148$

Hamilton, Joseph G., $32,56,66,90$, $111,115,123,128,130,135$

Helmholz, A. Carl, 75,103

Hilberry, Norman, 104,128, 129, 134

Hildebrana, Joel H. , 53

Hodges, J. H. , 93

Horn, W. R., 54

Johns, Iral B., Jr., 123,129

Kamen, Martin D. , 118,128

Katz, Joseph J., 127

Kennedy, Joseph w., $i, 1,3,5,10,11$, $12,14,16,17,20,21,22,30,31,32$, $33,34,36,38,41,44,48,60,64,65$, $67,68,69,72,73,77,83-84,89,111$, $117,124,127,128,129,134,136,141$ 
Kerst, D. W. , 111

Kharasch, Morris S., 104

Kohloss, F. H., 40

Kraus, Charles A., 114,127

Kurbatov, J. D., 103

Labine, Gilbert, 54,60

Langsdorf, Alexander, Jr , 146

Latimer, Wendell M., 24,83, 148

Lawrence, Ernest 0., 9,14,18,19, $35,42,43,44,49,53,60,73,74,80$, $82-83,89,94,95,114,115,116$, $130,131,134,137$

Leitz, Frederick J., ii, 3,60,80, 111

Lewis, Gilbert N., $14,17,18,35,40$, $44,45,55,119$

Libby, Willard F., 60,137

Livingston, M. Stanley, 94, 140

Long, Franklin A., 104, 111

Loomis, Alfred L., 43

Lundberg, Olaf, 52

Luttropp, Edward S., 21

Maier, C. G., 61

Manning, Winston M., 127

McCoy, Herbert N., 64, 104

McMillan, Edwin M. , 1,5,6,13,18, $19,20,31,35,43,124$

Meitner, Lise, 2

Miceli, A. S., 93

Murphree, Eger V., $83,127,136,140$, 143

Myers, F. E., 124

Nahinsky (Nahin), Paul G., 60,117 Norris, Thomas H., 60,74

Oliphant, Mark L., 60

Oppenheimer, J. Robert, 134

Orlemann, Edwin F., 60, 93

Patterson, Robert P., 35

Pegram, George B., 20,21

Perlman, Isadore, ii,83-84,85,86, $87,88,89,91,93,94,107,108,112$, $113,114-15,116,118,119,128,130$, $131,132,135,136,141,142,143$, $145,146,147,148-49$
Perlman, Morris I., ii, 61,65,66, $73,83-84,109,121,125,128,129$, $130,135,140-41,142,145,148$

Pochon, M. , $54,114,124,128,131$

Pool, M. L., 103

Prege1, Alexander, 111

Prestwood, Rene J., ii, 83,84,85, $86,87,88,89,91,93,94,103,107$, $108,113,118,119,132,138,146-47$, 148

Priest, Homer F., 137

Rice, C. N., 103

Roosevelt, Franklin D., 74

Ruben, Samuel, 60,103

Schlesinger, Herman I., 104

Seaborg, Helen Griggs, 123,124, 125,126

Segrè, Emilio, $i, 9,11,12,13,14,17$, $18,19,20,23,24,25,30,34-35,36$, $38,41-42,44,48,50,52,60,64,68$, $83,89,117,128,129,134$

Sheline, Glenn E., 136,138,140,141, 148

Slater, John C., 42

Spedding, Frank H., 114,115,116, $123,129,130,142$

Stewart, Irvin, 44,47,53

stoughton, Raymond $w ., i-i i, 56,57$, $60,61,62,64,65,66,69,70,75,78$, $80,83-84,85,86,87,90-91,92,98$, $101,102,106,108,113,115,117,118$, $119,121,124,128,143,144,145-46$, 147,148

Strassmann, Fritz, 2

Suito, E., 60

Szilard, Leo, 140,142

Tanzillo, Americo, 119

Tuve, Merle A., 19

Underhill, Robert M. , 44,47,52,55

Urey, Harold C. , 83, 94, 95, 114,115, $124,127,137,145$

Urry, W. D., 103

Van Atta, I. C., 124

Van Vleck, John H., 42 
Wahl, Arthur C., $i-i i, 1,3,4,6,7,9$, $10,11,12-13,14,15-16,17,20,22$, $23,24,25,26,27,30,31,32,33,34$, $35,36,37,38,39,40,41,43,44,45$, $46,47,48,49,50,51,53,54,55,56$, $57,58,59,60,61,62,63,64,65,67$, $68,72,73,74,75-76,77,78,79,80$, $81,82,83-84,85,87,88,89,92,94$, $96,97-98,100,101,103,105,106-07$, $108,109,111,116,118,120,123,124$, $125,126-27,128,134-35,136,137$, $139,143,145-46,148$

Wahl, Walter, 14

Weimer, P. K., 103

Wensel, H. T., 74,108,145

Wheeler, John A., 104,110

Wilke, R. M., 62

Winstein, Saul, 91

Yost, Don M., 67 